University of Tennessee Health Science Center

UTHSC Digital Commons

\title{
Using S. pombe to Study the Biological Roles of the Histone Deacetylases Sir2 and CIr3 and the DEAD-Box RNA Helicase Ded1
}

\author{
Brandon Ray Lowe \\ University of Tennessee Health Science Center
}

Follow this and additional works at: https://dc.uthsc.edu/dissertations

Part of the Chemicals and Drugs Commons, and the Neoplasms Commons

\footnotetext{
Recommended Citation

Lowe, Brandon Ray (http://orcid.org/0000-0002-4253-0494), "Using S. pombe to Study the Biological Roles of the Histone Deacetylases Sir2 and Clr3 and the DEAD-Box RNA Helicase Ded1" (2017). Theses and Dissertations (ETD). Paper 429. http://dx.doi.org/10.21007/etd.cghs.2017.0436.
}

This Dissertation is brought to you for free and open access by the College of Graduate Health Sciences at UTHSC Digital Commons. It has been accepted for inclusion in Theses and Dissertations (ETD) by an authorized administrator of UTHSC Digital Commons. For more information, please contact jwelch30@uthsc.edu. 


\title{
Using S. pombe to Study the Biological Roles of the Histone Deacetylases Sir2 and CIr3 and the DEAD-Box RNA Helicase Ded1
}

\begin{abstract}
The fission yeast Schizosaccharomyces pombe provides a good model system to quickly study basic mechanisms underlying biological pathways conserved in higher eukaryotes. Here we utilized fission yeast to study the roles of the histone deacetylases (HDACs) Sir2 and Clr3 in heterochromatin formation and cancer associated mutations of the DEAD-box RNA helicase DDX3X, homolog of fission yeast Ded1, in translational control. Heterochromatin in fission yeast is characterized by hypoacetylation as well as methylation of histone $\mathrm{H} 3$ on lysine 9 (H3K9me). Heterochromatin assembly can now be separated into three distinct steps: heterochromatin establishment, spreading, and maintenance. These steps involve the actions of the histone $\mathrm{H} 3 \mathrm{~K} 9$ methyltransferase $\mathrm{Clr} 4$ along with the RNAi pathway. HDACs are also required for heterochromatin assembly, with the H3K9 HDAC Sir2 and H3K14 HDAC CIr3 participating in the processes of heterochromatin establishment and maintenance. Here, we show that both a serine rich patch within the N-terminal domain of Sir2 and the HDAC activity of Sir2 are required for proper recruitment of Sir2 to chromatin and for heterochromatin establishment. We also report that Sir2 shares similarity in global transcriptional control with Mit1 and Chp2, components of the Snf2/HDAC-containing repressor complex (SHREC), suggesting a connection between Sir2 and SHREC in transcriptional regulation. We also identified additional sites that are deacetylated by the SHREC HDAC CIr3 in addition to H3K14, specifically H2B K5, 6, 10, and 15 . Using fission yeast, this study also examined the biological consequence of medulloblastoma associated mutations of the DEAD-box ATP-dependent RNA helicase DDX3X. In fission yeast, Ded1 is an essential protein with connections to both the RNAi pathway and translation of transcripts with complex UTRs. Here we show that human DDX3X can functionally complement for growth defects observed in thermosensitive ded1 mutants while specific cancerassociated DDX3X mutants (A222P, G302V, G325E, and P568L) cannot complement this growth defect. Fission yeast bearing these specific DDX3X mutants exhibit defects in the expression of specific proteins, suggesting the mutant proteins impair translational control.
\end{abstract}

\section{Document Type \\ Dissertation}

\section{Degree Name}

Doctor of Philosophy (PhD)

\section{Program}

Biomedical Sciences

Research Advisor

Janet F. Partridge, Ph.D.

Keywords

Clr3, DDX3X, Ded1, HDAC, Heterochromatin, Sir2

\section{Subject Categories}

Chemicals and Drugs | Diseases | Medicine and Health Sciences | Neoplasms

\section{Comments}

One year embargo expires May 2018. 
Using S. pombe to Study the Biological Roles of the Histone Deacetylases Sir2 and CIr3 and the DEAD-Box RNA Helicase Ded1

\author{
A Dissertation \\ Presented for \\ The Graduate Studies Council \\ The University of Tennessee \\ Health Science Center \\ In Partial Fulfillment \\ Of the Requirements for the Degree \\ Doctor of Philosophy \\ From The University of Tennessee
}

By

Brandon Ray Lowe

May 2017 
Portions of Chapter 4 (C) 2016 by Elsevier Inc.

All Other Material (C) 2017 by Brandon Ray Lowe.

All rights reserved. 


\section{DEDICATION}

This work is dedicated to my parents, Tim and Donna Lowe, who always encouraged me to never give up on my goals and to always believe in myself. 


\section{ACKNOWLEDGEMENTS}

I would like acknowledge all those whose practical contributions, guidance, and encouragement made this work possible. First and foremost, I would like to thank my mentor Dr. Janet Partridge for allowing me to study heterochromatin assembly in her lab. Without her mentorship, guidance, and support this work would not have been possible. I am truly grateful for your time and patience throughout my graduate studies. I would also like to thank both current and former member of the Partridge lab including Dr. Ben Alper, Dr. Rajesh Yadav, Dr. Alfonso Fernandez, Dr. Tao Xu, Dr. Kevin Creamer, Dr. Satish Kallappagoudar, Dr. Sreenath Shanker, Dr. Carolyn Jablonowski, and Godwin Job for their contributions to and helpful discussion of this work. I also want to thank Dr. Gerry Zambetti for his helpful suggestions regarding my research.

This work would not have been feasible without the generous contributions from all our collaborators. I would like to thank Dr. Alan Tackett and Dr. Stephanie Byrum at the University of Arkansas Medical School (UAMS) for performing MS/MS analysis of histones and Sir2. I would like to thank Dr. Thomas Schalch for working with us on SHREC. I would like to thank Dr. Andy Andrews and Dr. Ryan Henry at Fox Chase Cancer Center (FCCC) for their targeted MS/MS of histone acetylation. I would like to thank Dr. Eric Enemark and Leslie Epling at St. Jude Children's Research Hospital (SJCRH) for collaborative studies on the DDX3X project. I would like to thank Dr. Chunxu Qu (SJCRH) for her bioinformatic analysis. I would also like to thank the St. Jude Hartwell Center for all their services which made this work possible.

I would like to express my sincere appreciation to my committee members, Dr. Eric Enemark, Dr. Linda Hendershot, Dr. Nick Laribee, Dr. Lawrence Pfeffer, and Dr. Paul Brindle, for their thoughtful guidance, suggestions, and reading of this dissertation, which I truly appreciate.

I also want to thank St. Jude Children's Research Hospital, ALSAC, and The University of Tennessee Health Science Center for providing a great work and learning environment to complete my graduate education. Without these institutions and their financial support, this work would not be possible.

Finally, I would like to thank all my family and friends. Your words of encouragement and support were truly appreciated. I would especially like to thank my parents and grandparents who always believed I could accomplish my goals. 


\begin{abstract}
The fission yeast Schizosaccharomyces pombe provides a good model system to quickly study basic mechanisms underlying biological pathways conserved in higher eukaryotes. Here we utilized fission yeast to study the roles of the histone deacetylases (HDACs) Sir2 and Clr3 in heterochromatin formation and cancer associated mutations of the DEAD-box RNA helicase DDX3X, homolog of fission yeast Ded1, in translational control. Heterochromatin in fission yeast is characterized by hypoacetylation as well as methylation of histone $\mathrm{H} 3$ on lysine 9 (H3K9me). Heterochromatin assembly can now be separated into three distinct steps: heterochromatin establishment, spreading, and maintenance. These steps involve the actions of the histone $\mathrm{H} 3 \mathrm{~K} 9$ methyltransferase $\mathrm{Clr} 4$ along with the RNAi pathway. HDACs are also required for heterochromatin assembly, with the H3K9 HDAC Sir2 and H3K14 HDAC Clr3 participating in the processes of heterochromatin establishment and maintenance. Here, we show that both a serine rich patch within the N-terminal domain of Sir2 and the HDAC activity of Sir2 are required for proper recruitment of Sir2 to chromatin and for heterochromatin establishment. We also report that Sir2 shares similarity in global transcriptional control with Mit1 and Chp2, components of the Snf2/HDAC-containing repressor complex (SHREC), suggesting a connection between Sir2 and SHREC in transcriptional regulation. We also identified additional sites that are deacetylated by the SHREC HDAC Clr3 in addition to H3K14, specifically H2B K5, 6, 10, and 15. Using fission yeast, this study also examined the biological consequence of medulloblastoma associated mutations of the DEAD-box ATP-dependent RNA helicase DDX3X. In fission yeast, Ded1 is an essential protein with connections to both the RNAi pathway and translation of transcripts with complex UTRs. Here we show that human DDX3X can functionally complement for growth defects observed in thermosensitive dedl mutants while specific cancer-associated DDX3X mutants (A222P, G302V, G325E, and P568L) cannot complement this growth defect. Fission yeast bearing these specific DDX3X mutants exhibit defects in the expression of specific proteins, suggesting the mutant proteins impair translational control.
\end{abstract}




\section{TABLE OF CONTENTS}

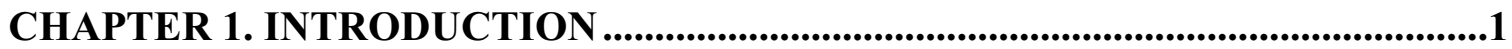

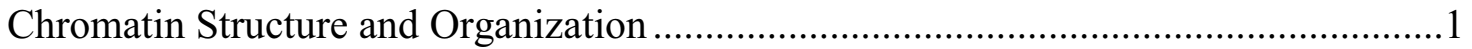

Post Translational Modification of Histones ............................................................ 1

Characteristics of Euchromatin and Heterochromatin ................................................2

Advantages of the Fission Yeast Model System .....................................................2

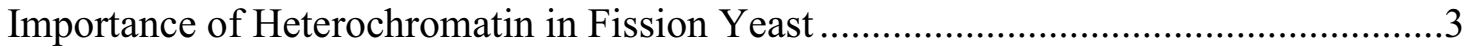

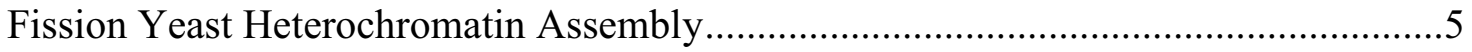

Clr4-Dependent H3K9 Methylation ...................................................................

Model for RNAi-Dependent Heterochromatin Formation ..................................6

Maintenance and Establishment of Centromeric Heterochromatin ............................8

RNAi Is Not Required for Establishment ...........................................................

Redundancy at Telomeres and Mating Type Locus............................................... 10

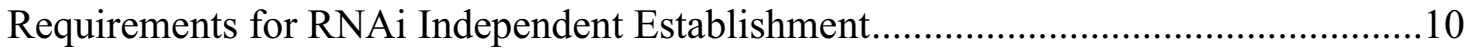

Role of HDACs in Heterochromatin Formation .................................................. 12

Redundancy of Sir2 and Clr3 in Heterochromatin Maintenance .............................12

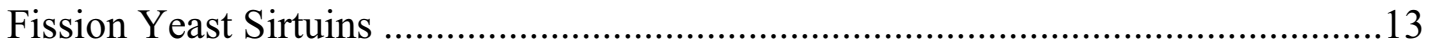

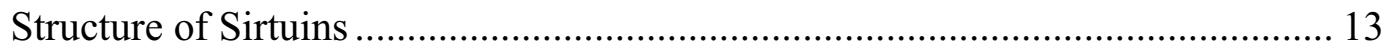

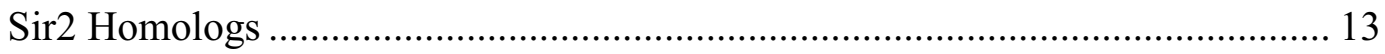

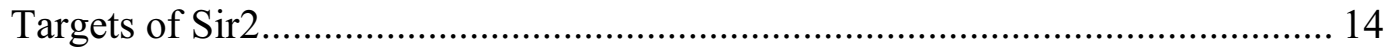

Sir2 Is Required for Centromeric Heterochromatin Establishment.................... 14

Roles of Sirtuin Auxiliary Domains ................................................................ 15

Aims to Understand the Role of Sir2 in Heterochromatin Assembly.................. 16

Role of Clr3 in Heterochromatin Assembly ............................................................. 16

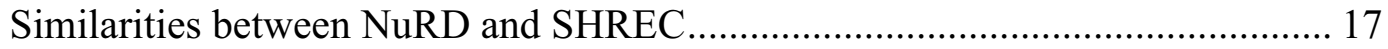

Role of SHREC at Heterochromatic Loci...................................................... 19

Aims to Understand the Role of Clr3 in Heterochromatin Assembly .................. 21

Fission Yeast as a Tool to Study Cancer Associated Mutations of DDX3X................21

DEAD-Box Helicases ............................................................................................. 21

Classification, Structure, and Mechanism of Action ..............................................24

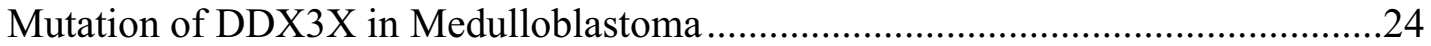

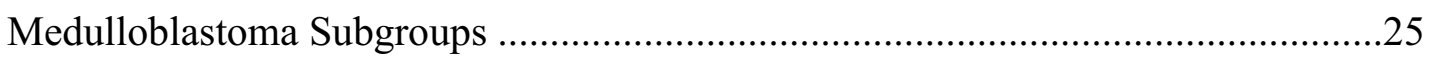

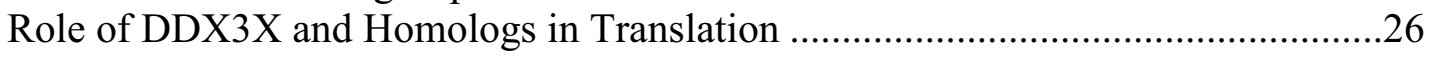

Aims for Examination of DDX3X Mutations...................................................27

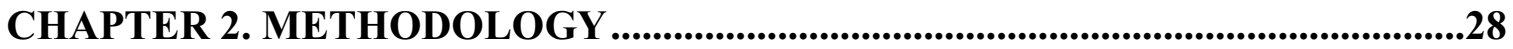

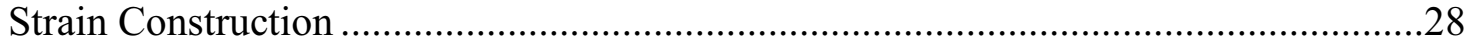

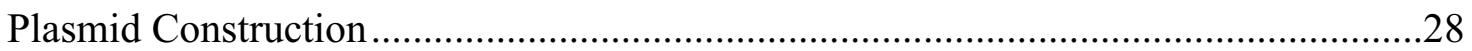

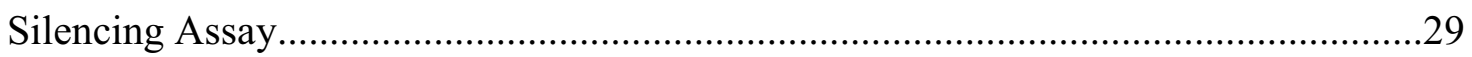

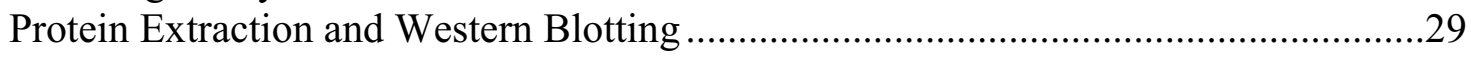

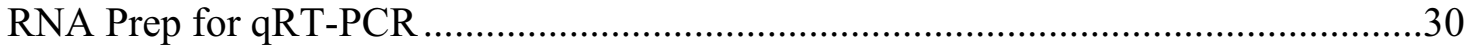

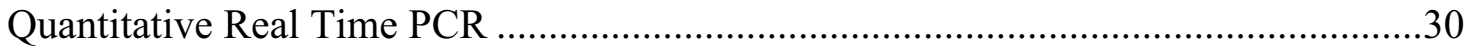

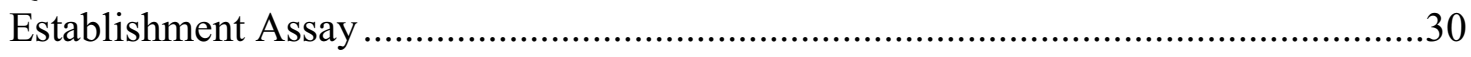

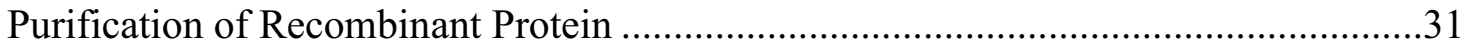




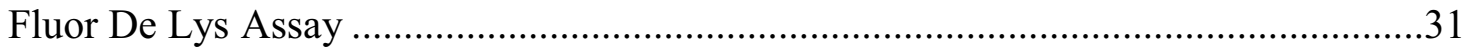

Histone Preparation and UPLC-MS/MS to Detect Acetylation ...................................32

Immunoprecipitation of Sir2 and MS/MS for Interactors and PTM .........................33

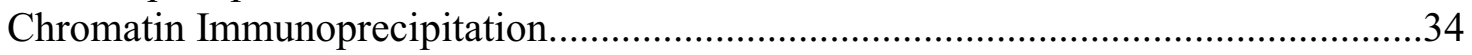

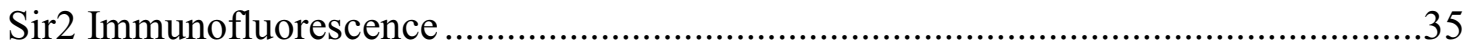

Electromobility Shift Assay ................................................................................. 35

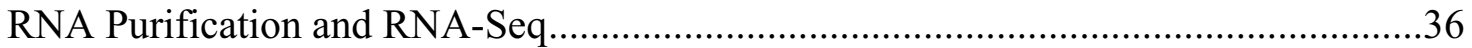

Histone Purification and MS/MS to Examine PTMs in clr3 $\Delta$ Cells .............................37

Measuring Ded1 Protein Levels ....................................................................... 38

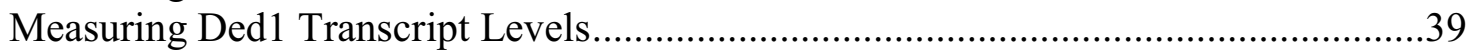

\section{CHAPTER 3. IMPORTANCE OF THE SIR2 NTD IN HETEROCHROMATIN} ESTABLISHMENT ..........................................................................................................40

Role of the Sir2 NTD in Heterochromatin Maintenance .............................................40

Sir2 $\Delta$ 1-129 Can Localize to the Nucleus..................................................................42

Role of the Sir2 NTD in Heterochromatin Establishment ............................................42

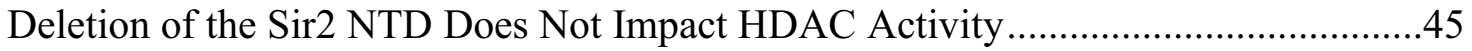

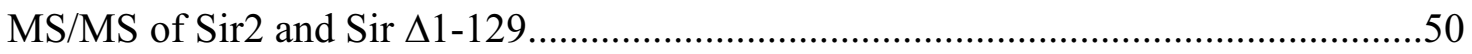

The Sir2 NTD Is Post-Translationally Modified ..................................................53

The Sir2 NTD Is Important for Chromatin Association ..........................................54

\section{CHAPTER 4. NOVEL TRANSCRIPTIONAL CONTROL AND TARGETS OF}

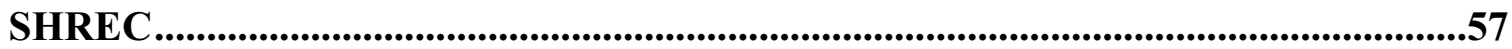

The Sir2 NTD Is Important for Redundancy with Clr3 …..........................................57

Separation of Function in Global Transcriptional Regulation by SHREC

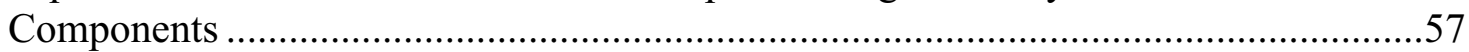

Sir2 Shows Overlap with the Mit1/Chp2 Sub-Complex ..............................................61

Clr3 Can Deacetylate Residues in the H2B N-Terminal Tail........................................62

Mutation of H2B K5, 6, 10, and 15 Does Not Impact Heterochromatin

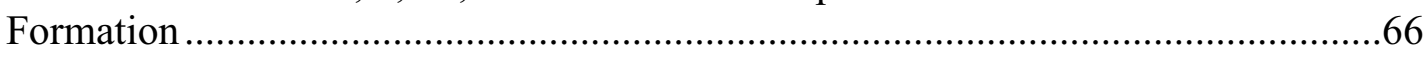

H2B 4Q Has Minimal Global Transcriptional Changes .............................................68

\section{CHAPTER 5. INVESTIGATION OF DDX3X MUTATIONS FOUND IN} PEDIATRIC MEDULLOBLASTOMA..................................................................71

Human DDX3X Can Functionally Complement for Fission Yeast Ded1 .....................71

Ded1 Is Not Involved in Silencing Pericentromeric Heterochromatin............................74

Some DDX3X Medulloblastoma Mutants Are Defective for Complementation...........74

Normal DDX3X Function Is Required for Cig2 Translation .......................................77

CHAPTER 6. DISCUSSION AND FUTURE DIRECTIONS .........................................80

The NTD and HDAC Activity of Sir2 Are Necessary for Establishment ......................80

The NTD of Sir2 Is Required for Proper Establishment..........................................8

Potential Sir2 Interactors: Insight from Sir2 Homologs ....................................... 83

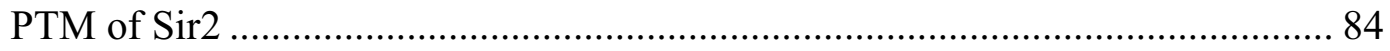

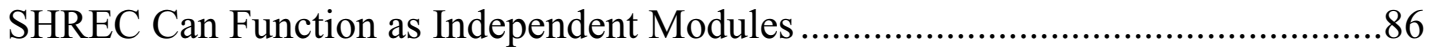


Sir2 Shows Overlap with the SHREC Remodeler Module...................................87

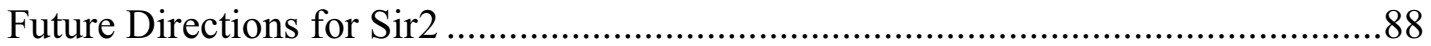

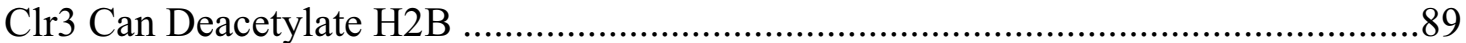

H2B N-Terminal Tail Affects Transcription ........................................................ 90

H2B N-Terminal Tail Affects Apoptotic-Like Death ...........................................90

Role of Clr3 in Nucleosome Stability .......................................................... 90

Future Directions for H2B Acetylation Study .................................................. 91

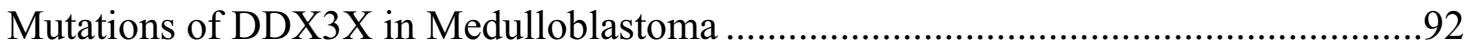

Consequences of DDX3X Mutation In Vitro ........................................................92

DDX3X Has a Conserved Role in Translation ......................................................93

DDX3X Targets 5'UTRs and Coding Regions in Humans ................................ 94

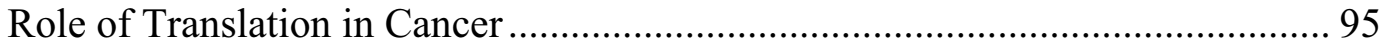

Possible Connection between DDX3X and Cancer................................................95

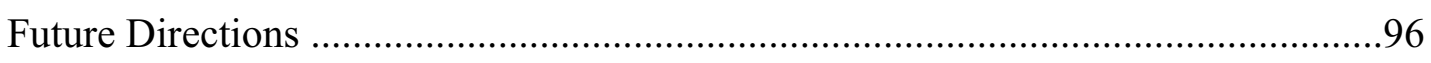

LIST OF REFERENCES ....................................................................................................97

APPENDIX. CHAPTER 2 SUPPLEMENTAL TABLES..............................................116

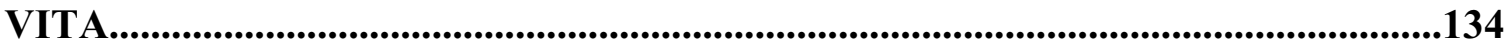




\section{LIST OF TABLES}

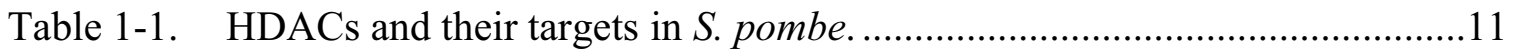

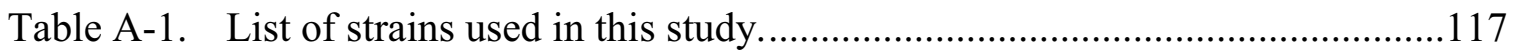

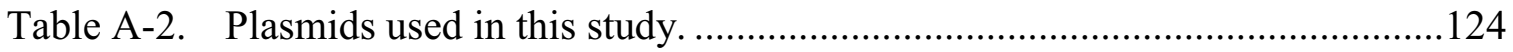

Table A-3. Detection parameters of unique tryptic peptides from pombe H3.............126

Table A-4. Sir2-3x V5 and Sir2 $\Delta 1-129-3 x$ V5 MS/MS results. .................................128

Table A-5. Phosphorylated Sir2 peptides obtained from LC-MS/MS ........................133 


\section{LIST OF FIGURES}

Figure 1-1. Heterochromatic loci in Schizosaccharomyces pombe ..................................

Figure 1-2. RNAi dependent heterochromatin formation in fission yeast.........................7

Figure 1-3. Comparison of the SHREC and NuRD complexes........................................18

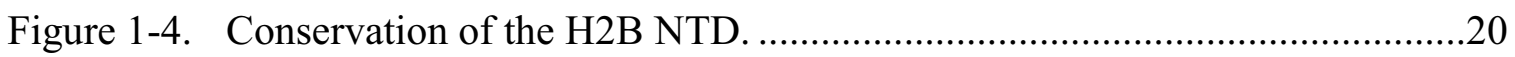

Figure 1-5. Domains and cancer associated mutants of Human DDX3X.....................22

Figure 3-1. The NTD and HDAC activity of Sir2 are important in heterochromatin maintenance.

Figure 3-2. The NTD and HDAC activity of Sir2 are important for heterochromatin establishment.

Figure 3-3. The serine rich patch of the Sir2 NTD is important for centromeric heterochromatin establishment.

Figure 3-4. The SSSG repeats are important for de novo centromeric heterochromatin establishment.

Figure 3-5. Deletion of residues 1-129 of Sir2 does not impact HDAC activity in vitro.

Figure 3-6. Deletion of residues 1-129 of Sir2 does not impact HDAC activity in vivo.

Figure 3-7. Phosphorylation of the Sir2 serine rich patch does not impact centromeric heterochromatin establishment......

Figure 3-8. The Sir2 NTD and HDAC activity are necessary for proper recruitment of Sir2 to chromatin

Figure 4-1. Sir2 $\Delta 1-129$ cannot maintain centromeric heterochromatin in the absence of Clr3.

Figure 4-2. SHREC subunits show separation of function and overlap with Sir2 in transcriptional regulation.

Figure 4-3. Sir2 deletion strains show overlaps in transcriptional regulation with both $\operatorname{chp} 2 \Delta$ and mit $1 \Delta$ strains.

Figure 4-4. Deletion of Clr3 alters recognition of H2B by a H2B NTD-specific antibody. 
Figure 4-5. Clr3 deacetylates H2B K5, 6, 10, and 15 in fission yeast.

Figure 4-6. The H2B K5, 6, 10, and 15 acetyl mimetic does not impact the maintenance or establishment of heterochromatin.

Figure 4-7. The H2B K5, 6, 10, and 15 acetyl mimetic has no major impact on transcriptional regulation.

Figure 5-1. DDX3X functionally complements for fission yeast ded1-1D5 temperature sensitive growth defect.

Figure 5-2. Specific DDX3X mutants cannot complement for loss of ded1-1D5 function...... .75

Figure 5-3. Defects in ded1-1D5 complementation by specific DDX3X mutants correlate with decreased Cig2 protein levels

Figure 5-4. Specific DDX3X mutants cannot complement the ded1-61 temperature sensitive defect. 78

Figure 6-1. Possible roles of the Sir2 NTD. .82 


\section{LIST OF ABBREVIATIONS}

\begin{tabular}{ll} 
ABL & ATP Binding Loop \\
ac & Acetylation \\
adh1 & Alcohol Dehydrogenase 1 \\
Ago1 & Argonaute 1 \\
ARC & Argonaute siRNA Chaperone Complex \\
BP & Base Pairs \\
CD & Chromodomain \\
cenH & Centromere Homologous Domain \\
CENP-A & Centromere Protein A \\
ChIP & Chromatin Immunoprecipitation \\
Chp & Chromodomain Protein \\
CID & Collision Induced Dissociation \\
Cig2 & G1/S Cyclin \\
Clr & Cryptic Loci Regulator \\
CLR-C & Clr4 Methyltransferase Complex \\
cnt & Central Core Domain of Centromere \\
CTD & Carboxy Terminal Domain \\
DAPI & 4',6'-Diamidino-2-phenylindole \\
Dcr1 & Dicer 1 \\
DDX3X & DEAD-Box Helicase 3, X-Linked \\
DEPC & Diethylpyrocarbonate \\
$d g$ & Repetitive Element in otr \\
$d h$ & Repetitive Element in otr \\
DNA & Deoxyribonucleic Acid \\
DOC & Deoxycholate \\
dsDNA & Double Stranded Deoxyribonucleic Acid \\
dsRNA & Double Stranded Ribonucleic Acid \\
DTT & Dithiothreitol \\
EDTA & Ethylenediaminetetraacetic Acid \\
EGTA & Ethylene Glycol-bis(oxyethylenenitrilo)tetraacetic Acid \\
GST & Glutathione S-transferase \\
HAT & Histone Acetyltransferase \\
HBR & H2B Repression Domain \\
HCD & High-Energy Collisional Dissociation \\
HDAC & Histone Deacetylase \\
HMT & Histone Methyltransferase \\
HP1 & Heterochromatin Protein 1 \\
Hst & Homologue of Sir2 \\
H2B 4Q & H2B K5, 6, 10, 15Q Acetyl Mimetic \\
H2B 4R & H2B K5, 6, 10, 15R Deacetyl Mimetic \\
$i m r$ & Centromeric Innermost Repeats \\
KanR & Kanamycin Resistance \\
Kb & \\
& Kilo-base \\
\hline ind &
\end{tabular}




$\begin{array}{ll}\text { KDAC } & \text { Lysine Deacetylase } \\ \text { LC-MS/MS } & \text { Liquid Chromatography-Tandem Mass Spectrometry } \\ \text { MAD } & \text { Median Absolute Deviation } \\ \text { mat } & \text { Mating Type Locus } \\ \text { Mb } & \text { Megabase } \\ \text { MBDL } & \text { Methyl-CpG binding-like domain } \\ \text { me } & \text { Methylation } \\ \text { Mit1 } & \text { Mi-2-like Interacting with Clr3 Protein 1 } \\ \text { MS/MS } & \text { Tandem Mass Spectrometry } \\ \text { mRNA } & \text { Messenger RNA } \\ \text { NAD } & \text { Nicotinamide Adenine Dinucleotide } \\ \text { NFR } & \text { Nucleosome Free Region } \\ \text { NMR } & \text { Nuclear Magnetic Resonance Spectroscopy } \\ \text { NTD } & \text { Amino Terminal Domain } \\ \text { NuRD } & \text { Nucleosome Remodeling Deacetylase } \\ \text { otr } & \text { Centromeric Outer Repeats } \\ \text { PAGE } & \text { Polyacrylamide Gel Electrophoresis } \\ \text { PBS } & \text { Phosphate Buffered Saline } \\ \text { PCGP } & \text { Pediatric Cancer Genome Project } \\ \text { PCR } & \text { Polymerase Chain Reaction } \\ \text { PEV } & \text { Position Effect Variegation } \\ \text { PHD } & \text { Plant Homeo Domain } \\ \text { PMG } & \text { pombe Minimal Glutamate } \\ \text { PMSF } & \text { Phenylmethylsulfonyl Fluoride } \\ \text { PTM } & \text { Post Translational Modification } \\ \text { qRT-PCR } & \text { Quantitative Realtime PCR } \\ \text { RDRC } & \text { RNA-Dependent RNA Polymerase Complex } \\ \text { RDRP } & \text { RNA-Dependent RNA Polymerase } \\ \text { RITS } & \text { RNA-induced Initiation of Transcription Silencing Complex } \\ \text { RNA } & \text { Ribonucleic Acid } \\ \text { RNAi } & \text { Ribonucleic Acid Interference } \\ \text { RNA Pol II } & \text { Ribonucleic Acid Polymerase II } \\ \text { RPM } & \text { Revolutions Per Minute } \\ \text { RSC } & \text { Chromatin Structure Remodeling Complex } \\ \text { SDS } & \text { Sodium Dodecyl Sulfate } \\ \text { SEM } & \text { Standard Error of the Means } \\ \text { SHREC } & \text { Snf2/Histone Deacetylase Repressor Complex } \\ \text { siRNA } & \text { Small Interfering Ribonucleic Acid } \\ \text { Sir2 } & \text { Trilent Information Regulator 2 } \\ \text { Sir2 N247A } & \text { Silent Information Regulator 2 Catalytic Mutant } \\ \text { sir2A } & \text { Sir2 Establishment Strain } \\ \text { ssDNA } & \text { Single Stranded Deoxyribonucleic Acid } \\ \text { ssRNA } & \text { Single Stranded Ribonucleic Acid } \\ \text { Tas3WG } & \text { Tas3 Lacking Ago1 Interaction Hook } \\ \text { TBE } & \text { Tris } \\ \text { TE } & \end{array}$


TES

TGS

thl 1

UHC

UTR

WCE

XIST

YES

5-FOA
Tris/EDTA Buffer with SDS

Transcriptional Gene Silencing

Gene within Subtelomeric Repeats

Unsupervised Hierarchical Clustering

Untranslated Region

Whole Cell Extract

X-Inactive Specific Transcript

Yeast Extract with Supplements

5-Fluoroorotic Acid 


\section{CHAPTER 1. INTRODUCTION}

\section{Chromatin Structure and Organization}

The large genome of eukaryotic organisms is highly organized and compacted into a structure known as chromatin to fit within the cell. Chromatin consists of deoxy ribonucleic acid (DNA) which interacts with both histone and non-histone proteins, allowing for high levels of compaction. The basic unit of chromatin consists of nucleosomes which are made up of around 147 base pairs (bp) of DNA wrapped around an octameric histone core consisting of two copies of each of the four core histones: $\mathrm{H} 2 \mathrm{~A}, \mathrm{H} 2 \mathrm{~B}, \mathrm{H} 3$, and H4. Nucleosome assembly is regulated by histone chaperones which guide their deposition onto DNA after DNA replication $[1,2]$. The most basic level of chromatin is known as the "beads on a string" model which consists of nucleosomes separated by short segments of linker DNA [3]. Additional compaction leads to the formation of the $30 \mathrm{~nm}$ fiber and eventually, the maximally compacted metaphase chromosome.

\section{Post Translational Modification of Histones}

It is now understood that the heritable physical traits exhibited by an organism are more complex than simple changes within the gene sequence encoding them. In addition to the gene sequence, the packaging of DNA also plays a crucial role in determining phenotypic characteristics. This knowledge laid the foundation for our current understanding of the complex processes guiding epigenetics. Over a half century ago, it was discovered that histones themselves undergo post-translational modification (PTM) with the finding that calf-thymus histones are acetylated and this mark can regulate transcription $[4,5]$. We now know there are a multitude of PTMs including acetylation, methylation, phosphorylation, ubiquitination, and sumoylation which occur mainly on the $\mathrm{N}$-terminal tail of histones, an area which protrudes from the globular histone core and is rich in basic residues [6].

PTMs are crucial in the process of regulating gene expression without changes to the actual gene sequence by dictating the state of chromatin compaction. For example, acetylation of histone N-terminal tails by histone acetyltransferases (HATs) weakens the interaction of the negatively charged DNA with the positively charged histone residues, leading to decreased chromatin compaction and increased accessibility to the transcriptional machinery [7]. Acetylation can also recruit factors containing a bromodomain which binds to the acetyl mark and further modify chromatin [8]. Another key PTM is methylation of histone tails, catalyzed by histone methyltransferases (HMTs), which frequently contain a Suvar39, Enhancer of Zeste and Trithorax (SET) domain necessary for enzymatic activity along with a cofactor, $S$-adenosyl-L-methionine. This mark can recruit factors involved in altering chromatin compaction and transcription $[6,9-12]$. Although much has been learned since the initial discovery of histone PTM, 
combinations of PTMs and their role in transcriptional regulation are still being dissected as new modifications and combinations of modifications continue to be discovered.

\section{Characteristics of Euchromatin and Heterochromatin}

In the late 1920's, the work of Emil Heitz examining the chromatin of moss gave initial evidence to the idea of two distinct states of chromatin now known as euchromatin and heterochromatin. He discovered that during interphase, chromatin stains

differentially and this differential staining is based on the level of chromatin compaction. The term heterochromatin was used to describe regions which remain condensed and intensely stained in contrast to euchromatin, which was less condensed and lightly stained [13]. Since this time, new research has given greater insight into understanding the differences between euchromatin and heterochromatin. Euchromatin is the more relaxed and open state of chromatin, more accessible to transcriptional machinery and consisting mainly of actively transcribed regions of the genome. It is characterized by methylation of lysine 4 of histone $\mathrm{H} 3(\mathrm{H} 3 \mathrm{~K} 4 \mathrm{me}$ ) along with hyperacetylation of the histone tails which contributes to its open state. In contrast, heterochromatin is the more compacted chromatin form which is generally less accessible to the transcriptional machinery within the cell and consists mainly of inactive genes and genomic structural elements. Heterochromatin is characterized by hypoacetylation along with methylation on lysine 9 of histone H3 (H3K9), lysine 27 of histone H3 (H3K27), and lysine 20 of histone $\mathrm{H} 4$ (H4K20). HP1 proteins which bind to $\mathrm{H} 3 \mathrm{~K} 9 \mathrm{me}$ are also a defining feature of heterochromatin $[6,9,14,15]$.

There are two main types of heterochromatin: facultative heterochromatin and constitutive heterochromatin. Facultative heterochromatin has also been referred to as regulated heterochromatin since it mainly assembles on genes which are active during some point in development or the cell cycle $[14,16,17]$. One of the most common examples of facultative heterochromatin involves the non-coding ribonucleic acid (RNA) termed the X-inactive specific transcript (XIST), which plays a key role in Xchromosome inactivation during early development $[16,18]$. In contrast, constitutive heterochromatin, as the name implies, is the more permanent form of heterochromatin which consists mainly of structural elements of the genome such as telomeres and centromeres. This type of heterochromatin tends to assemble at areas where there are highly repetitive sequences within the genome like transposons, telomeric repeats, and satellite repeats found at centromeres. Heterochromatin protects these regions from unwanted rearrangement or mutation $[14,19]$. In spite of considerable research efforts to dissect the mechanism and factors involved in heterochromatin assembly and propagation, there remains many unanswered questions.

\section{Advantages of the Fission Yeast Model System}

The fission yeast Schizosaccharomyces pombe provides a good system to study the processes involved in the assembly and maintenance of constitutive heterochromatin. 
Position effect variegation (PEV) was first discovered in Drosophila melanogaster with the observations that when reporter genes which are normally transcriptionally active are translocated next to heterochromatic regions, they subsequently become inactive due to heterochromatic spreading [24]. Based on these observations, reporter genes were inserted into putative heterochromatic domains in fission yeast and transcriptional silencing was observed [14, 20-24]. Since then, fission yeast have become an increasingly valuable tool in dissecting the mechanisms governing heterochromatin formation and maintenance, often through the use of reporter genes to study these processes. Fission yeast offer several distinct advantages as tools to study heterochromatin in contrast to higher eukaryotes. The fission yeast genome is smaller than that of metazoans, containing around 5000 genes within a $13.8 \mathrm{Mb}$ genome contained on three large chromosomes [22,25]. Unlike higher eukaryotes with complex genomes and proteins which often perform redundant functions, fission yeast provide a simple model where many factors which regulate chromatin structure and function are conserved and present in a single copy $[14,22]$. Fission yeast also provide a highly genetically tractable system, allowing for the study of genes through deletion, mutation, and autonomous plasmid expression to quickly access their biological function due to a relatively short division time of only 2.5 hours in rich media [22, 26, 27]. In addition, structural elements of the genome such as centromeres bear similarity to those of higher eukaryotes, providing a platform on which to study the basic processes guiding chromatin structure [14]. These advantages coupled with the relative low cost of maintenance have made fission yeast a valuable tool in dissecting the processes which underlie the formation of chromatin structure.

\section{Importance of Heterochromatin in Fission Yeast}

In fission yeast, there are three main regions of constitutive heterochromatin: telomeres, mating type locus (mat), and pericentromeres (Figure 1-1). Fission yeast telomeres share similarity with those of higher eukaryotes, containing arrangements important for telomere pairing and recombination which are absent in Saccharomyces cerevisiae (budding yeast) [28]. In fission yeast, the chromosome ends contain telomeric repeats along with subtelomeric regions stretching over $20 \mathrm{~Kb}$. Portions of the telomeres and most of the subtelomeric regions are coated in heterochromatin $[14,28]$. The mating type region consists of a $20 \mathrm{~Kb}$ region which is coated in heterochromatin in order to prevent recombination and allow for determining the mating type of the cell, with fission yeast having two mating types: $h+$ and $h-[22,29]$. In contrast to budding yeast, which have a $125 \mathrm{bp}$ point centromere, fission yeast have large, complex centromeres surrounded by repetitive pericentric elements similar to those of higher eukaryotes ranging from 40 to $110 \mathrm{~Kb}$ in size $[14,25,30]$. The centromere itself consists of a central core (cnt) which serves as the site of kinetochore assembly during mitosis and meiosis, directly surrounded by the innermost inverted repeats (imr). Within the central core, H3 is replaced with the $\mathrm{H} 3$ variant Cnp1 (homolog of CENP-A) which aids in directing kinetochore assembly. This is surrounded by the outer most repeats (otr), which consists of a series of repetitive $d g$ and $d h$ repeats which vary in number between the three chromosomes and are entirely coated in heterochromatin [14, 30-32]. All three regions of 

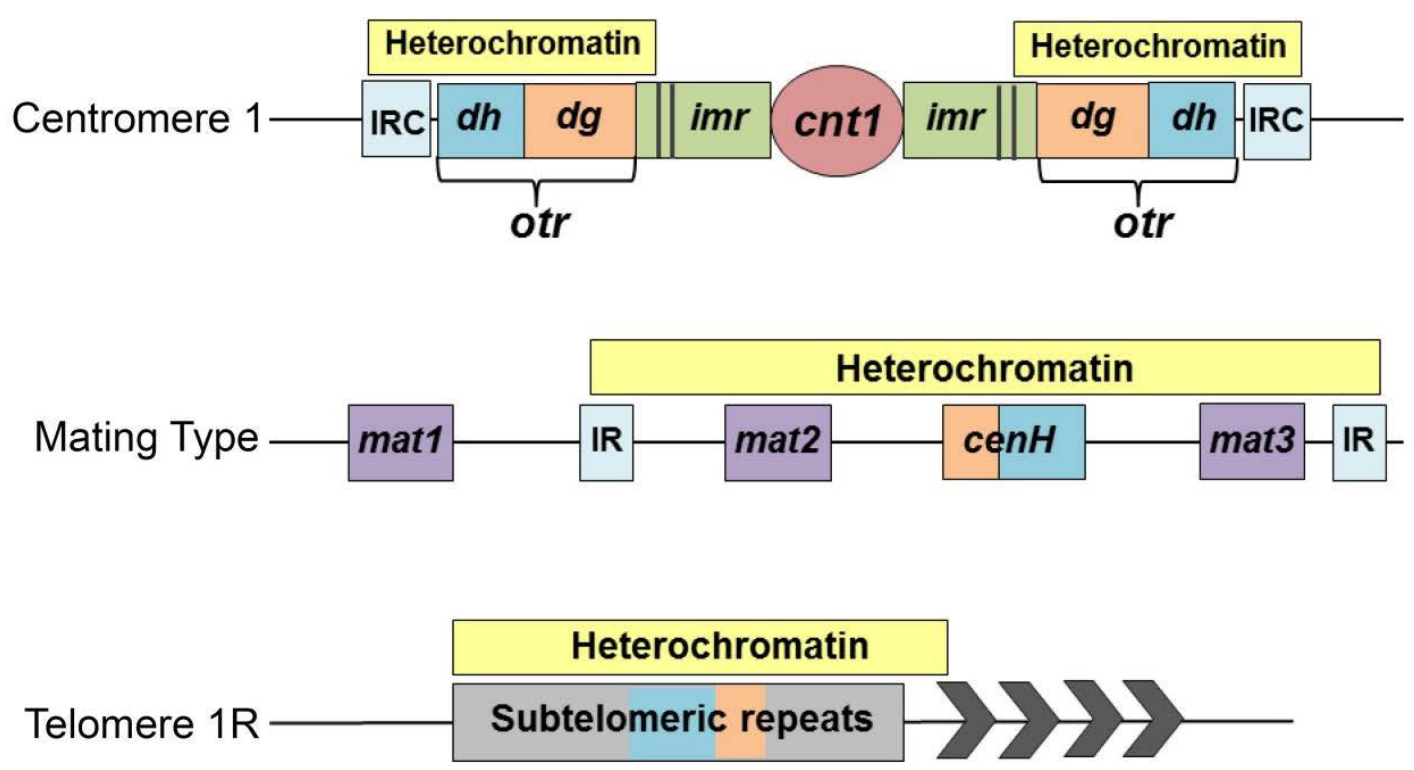

Figure 1-1. Heterochromatic loci in Schizosaccharomyces pombe.

Fission yeast have three heterochromatic loci: centromeres, the mating type locus (mat), and telomeres. At the centromere, the central core (cnt 1$)$ is surrounded by the large inverted repeats of the imr and otr. The otr contains the $d g$ and $d h$ repeats, which are important for recruiting the RNAi machinery to the centromeric repeats. Heterochromatin coats the entire otr region along with portions of the imr and IRC elements. At mat, the mat 2 and mat 3 loci are coated in heterochromatin. This region contains a cenH element which has repetitive elements similar to the $d g$ and $d h$ repeats of the centromere and is important for RNAi-dependent heterochromatin formation. At the telomere, the large subtelomeric repeats are coated in heterochromatin. Like mat, this region contains repetitive elements similar to the $d g$ and $d h$ repeats important for RNAi-dependent heterochromatin formation. 
heterochromatin contain repetitive elements similar to the $d g$ and $d h$ repeats found at pericentromeric regions which play a role in heterochromatin assembly [14].

Heterochromatin also forms on ribosomal DNA (rDNA), but the purpose of this remains unknown in fission yeast $[22,33]$. Our research is primarily focused on the initial steps required for heterochromatin assembly at the centromere, a process in which many questions remain unanswered.

Disruption of proper chromosome segregation can lead to aneuploidy where the daughter cells lack the correct number of each chromosome. This can have serious consequences in higher eukaryotes such as aiding in the development of cancer or causing a miscarriage during pregnancy [34]. In fission yeast, intact pericentromeric heterochromatin is necessary for proper chromosome segregation during anaphase of the cell cycle. When components necessary for the formation of pericentromeric heterochromatin are deleted or inhibited, increased loss of a "minichromosome" derived from chromosome 3 was observed. Also, a high rate of lagging chromosomes and missegregation events were seen when cells with disrupted pericentromeric heterochromatin were examined using fluorescent microscopy [21, 35-37]. It is now believed that pericentromeric heterochromatin is important for forming higher order structures which allow the cnt to be held in the proper orientation for kinetochore assembly and microtubule attachment during the cell cycle [38]. Pericentromeric heterochromatin is also important for the recruitment of cohesin, a protein complex essential for holding sister chromatids together until proper separation during anaphase. Cohesin is recruited to areas of centromeric heterochromatin through an interaction of the Psc3 subunit with Swi6, the fission yeast HP1 homolog [39-41]. These studies into the functional role of pericentromeric heterochromatin in fission yeast highlight its biological significance and the importance of understanding its formation.

\section{Fission Yeast Heterochromatin Assembly}

Like heterochromatic regions of higher eukaryotes, heterochromatin at the three heterochromatic loci in fission yeast is characterized by hypoacetylation along with methylation of $\mathrm{H} 3 \mathrm{~K} 9 \mathrm{me}$, although the pathway responsible for $\mathrm{H} 3 \mathrm{~K} 27 \mathrm{me}$ is not conserved [42]. The hypoacetylation and $\mathrm{H} 3 \mathrm{~K} 9$ me mark promotes further $\mathrm{H} 3 \mathrm{~K} 9$ methylation and the recruitment of members of the RNA interference (RNAi) pathway which is important for the formation and spreading of heterochromatin in fission yeast discussed below.

\section{Clr4-Dependent H3K9 Methylation}

Unlike higher eukaryotes where multiple HMTs can deposit H3K9me, fission yeast have a sole H3K9 HMT, cryptic loci regulator $4(\mathrm{Clr} 4)$, responsible for mono, di, and trimethylation of H3K9 [9]. Clr4 is the homolog of the SUV39 family of HMTs in higher eukaryotes. Like the SUV39 family of HMTs, Clr4 contains a cysteine rich preSET domain which contributes to the structural stability of the SET domain. The 
conserved post-SET domain contains conserved cysteine residues which contribute to active site interactions $[11,43,44]$. Once deposited, H3K9me can be bound by proteins which contain a chromodomain (CD) $[45,46]$. Fission yeast have four CD containing proteins which recognize the H3K9me mark: the HP1 homologs Swi6 and Chp2 along with Chp1, and Clr4 itself. These proteins serve to recruit factors important for assembling and maintaining heterochromatin [14, 47, 48]. The simplistic nature of heterochromatin in fission yeast makes it a prime model to study the events involved in the establishment and maintenance of heterochromatin.

Clr4 acts in a complex known as Clr-C with four other members: Rik1, Raf1, Raf2, and Pcu4. The Clr-C complex is part of an active cullin 4-dependent E3 ubiquitin ligase, although the ubiquitin ligase role is currently unclear [31, 49-52]. While the exact functions of all Clr-C components are not well understood, it is known that all components are necessary for proper formation of heterochromatin and loss of these components leads to loss of all $\mathrm{H} 3 \mathrm{~K} 9$ me at pericentromeric repeats [31]. One function of Rik1, along with Dos1, is to interact with the H3K4 demethylase Lid2 allowing for H3K4 demethylation at the pericentromere $[53,54]$. Clr4 itself can bind to the H3K9me mark through its $\mathrm{CD}$, leading to amplification of the $\mathrm{H} 3 \mathrm{~K} 9 \mathrm{me}$ signal by further recruitment of $\mathrm{Clr}-\mathrm{C}$ and $\mathrm{Clr}-\mathrm{C}$ can also be recruited through members of the RNAi pathway [55]. This allows for the spreading of heterochromatin over the defined domain.

\section{Model for RNAi-Dependent Heterochromatin Formation}

Constitutive heterochromatic regions at fission yeast centromeres are typically thought of as being transcriptionally silent due to the blocking of RNA polymerase II (RNA pol II) access by inhibitory marks such as $\mathrm{H} 3 \mathrm{~K} 9 \mathrm{me}$ in a process known as transcriptional gene silencing (TGS). It is now known that this theory is not completely correct and there is low level transcription of these repetitive regions [56, 57]. Supporting this observation, it has been shown that mutations in transcriptional elongation factors and RNA pol II cofactors leads to reduced $\mathrm{H} 3 \mathrm{~K} 9 \mathrm{me}$ and Swi6 association at centromeres $[58,59]$. The low-level transcription necessary for heterochromatin formation occurs during $\mathrm{S}$ phase of the cell cycle $[56,57,60,61]$. As DNA is being replicated, new nucleosomes are incorporated onto the newly synthesized DNA, diluting out Swi6 and repressive PTMs such as $\mathrm{H} 3 \mathrm{~K} 9$ me along with the appearance of acetylation. This allows RNA pol II temporary access to these normally inaccessible pericentromeric repeats [31, $60,61]$. The centromeric transcripts are targeted and destroyed by the RNAi pathway through post-transcriptional gene silencing (PTGS) before feeding into the RNAi pathway where they play a crucial role in heterochromatin assembly (Figure 1-2) [14, 22, $62]$.

The single stranded RNA (ssRNA) produced during S phase is converted into double stranded RNA (dsRNA) through the actions of RNA Dependent Polymerase (Rdp1) of the RNA Dependent RNA Polymerase (RDRC) complex along with Hrr1, a predicted RNA helicase, and Cid12, a polyA polymerase (Figure 1-2) [63-65]. The resulting dsRNA transcripts are cleaved by the conserved RNAse III endonuclease Dicer 


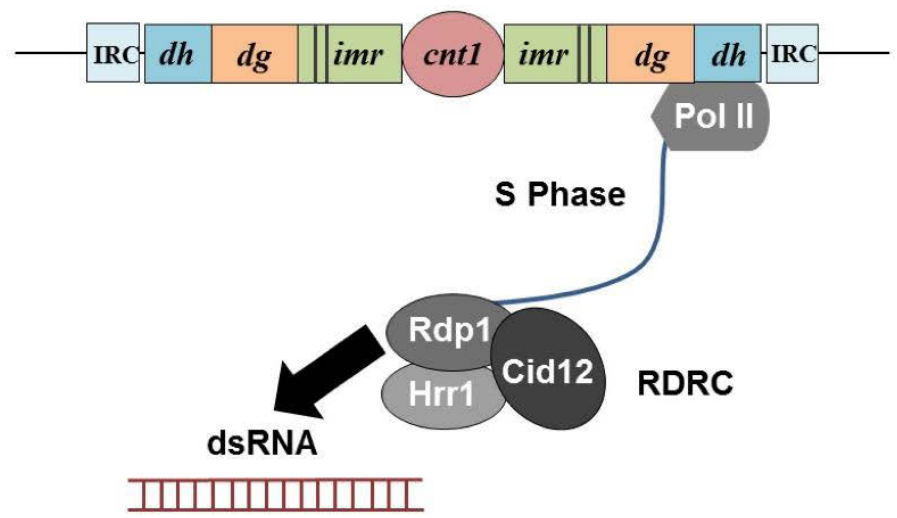

\section{Dcr1}

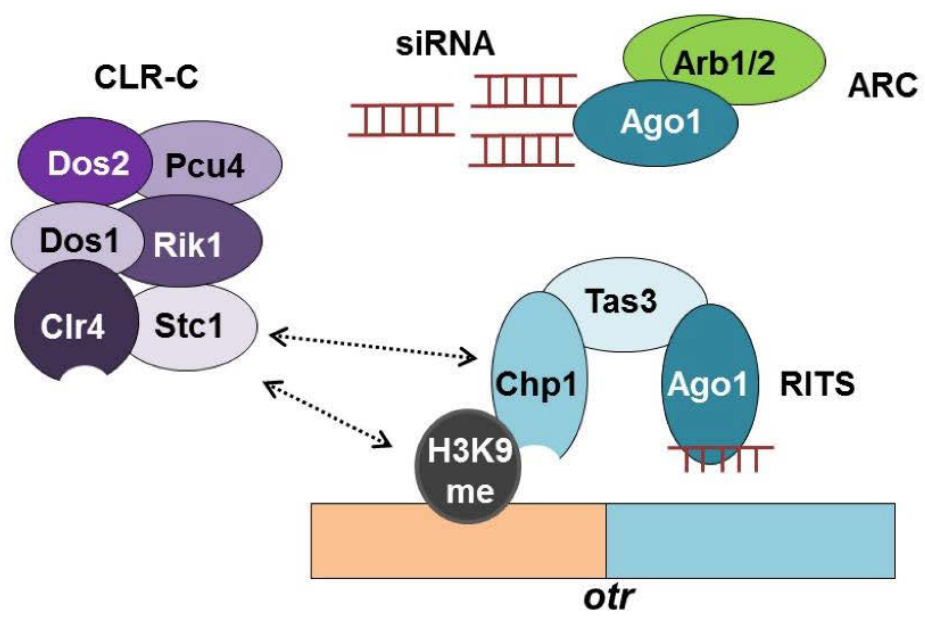

Figure 1-2. RNAi dependent heterochromatin formation in fission yeast.

During S phase of the cells cycle, the centromeric repeats are transcribed at low levels by RNA Pol II. The complementary strand is synthesized by RNA-dependent RNA polymerase of the RDRC complex and cleaved into siRNAs by Dcrl. These siRNAs are bound by Ago1 of the Arc complex before Ago1 is transferred to the RITS complex. Once Agol associates with the RITS complex, it cleaves the "passenger" strand of the siRNA. The siRNAs bound by Agol along with the Chp1 CD guide RITS to the centromeric repeats. The Clr-C complex is recruited through interactions with RITS along with recruitment by the $\mathrm{Clr} 4 \mathrm{CD}$, leading to a self-reinforcing loop of $\mathrm{H} 3 \mathrm{~K} 9 \mathrm{me}$ and heterochromatin spreading over the defined domains. 
(Dcr1) into short interfering RNAs (siRNAs) of around 21 nucleotides in length $[22,65$, 66]. These ds siRNAs, which are generated from highly specific regions of the otr, are then bound by Argonaute 1 (Ago1) of the Argonaute complex (ARC) along with the Argonaute binding proteins 1 and 2 (Arb1/2). The ribonuclease activity of Agol is blocked until Agol in complex with the ds siRNA is transferred to the RNA-induced initiation of transcription complex (RITS) [65].

RITS is a complex which consists of Ago1, Chp1, and Tas3, with Tas3 serving as a bridge between Ago1 and Chp1 [67-69]. Once Agol containing the ds siRNA is transferred to the RITS complex, Agol then cleaves the "passenger" strand through the endonuclease activity of its conserved PIWI domain $[65,66]$. RITS is then guided to the pericentromeric repeats through the ssRNA associated with Agol and the ability of Chpl to bind $\mathrm{H} 3 \mathrm{~K} 9 \mathrm{me}$ through its CD. RITS also serves as a bridge between RNAi and Clr-C through further recruitment of the Clr-C complex through interactions between Rik1 and RITS [55]. More recently, it was discovered that RITS can also interact with Clr-C via a LIM domain protein, Stc1, which serves as a bridge between Ago1 of RITS and components of $\mathrm{Clr}-\mathrm{C}[52,70,71]$. These interactions create a circular feedback loop which leads to further $\mathrm{H} 3 \mathrm{~K} 9 \mathrm{me}$ and spreading of heterochromatin at the pericentromeric repeats (Figure 1-2). Although $\mathrm{H} 3 \mathrm{~K} 9$ me plays a crucial role in constitutive heterochromatin assembly in mammals, whether RNAi contributes to this process remains unclear though much of the RNAi machinery is conserved [72]. Research has shown that transcripts from the heterochromatic pericentromeric satellite repeats can be detected in some mammalian cells [72-76]. Some evidence suggests these transcripts are important for HP1 $\beta$ recruitment to pericentromeric satellite repeats during development but no direct links to RNAi have been found $[72,77]$. Due to the conservation of the RNAi components, insights in to fission yeast heterochromatin assembly will likely aid in understanding the basic processes guiding heterochromatin formation in higher eukaryotes.

Deletion or mutation of components of the fission yeast RNAi pathway disrupts pericentromeric heterochromatin with increased levels of pericentromeric repeat transcripts, loss of siRNAs, and decreased H3K9me. Deletions or mutations in RITS also leads to a disruption of pericentromeric heterochromatin similar to RNAi mutants with high levels of pericentromeric transcripts and decreased H3K9me2 [56, 67, 69, 78]. Although $\mathrm{H} 3 \mathrm{~K} 9 \mathrm{me}$ is decreased in RNAi or RITS mutants, they retain some H3K9me in contrast to a $\operatorname{clr} 4 \Delta$ strain which shows a loss of all H3K9me $[78,79]$. These observations have contributed to a model in which heterochromatin establishment can occur independent of RNAi in fission yeast.

\section{Maintenance and Establishment of Centromeric Heterochromatin}

The study of proteins involved in heterochromatin can now be divided into two distinct steps: maintenance of heterochromatin and establishment of heterochromatin. Heterochromatin maintenance is the process of maintaining heterochromatin which has already been established throughout the cell cycle and cell division. The initial H3K9 
methylation events necessary for heterochromatin formation have already occurred and resulted in heterochromatin spreading over defined domains. Maintenance of heterochromatin requires both RNAi and RITS to recruit $\mathrm{Clr}-\mathrm{C}$ to the pericentromeric repeats, allowing for restoration of the already established heterochromatic domains [22].

Heterochromatin establishment, in which the process of the initial de novo deposition of $\mathrm{H} 3 \mathrm{~K} 9 \mathrm{me}$ is examined, has been more difficult to study. The proteins and pathways leading to the initial $\mathrm{H} 3 \mathrm{~K} 9$ me deposition in fission yeast remain controversial. It was first believed that the initial H3K9me events required both RNAi and RITS, following a pathway similar to heterochromatin maintenance which utilizes the process of PTGS. There are three main methods which have been used to study heterochromatin establishment. One method relies on the use of the HDAC inhibitor Trichostatin A, leading to inhibition of $\mathrm{H} 3 \mathrm{~K} 9 \mathrm{me}$ due to an increase in acetylation [80]. Another method to examine establishment of heterochromatin relies on a plasmid which contains a "minichromosome." These plasmids contain centromeric regions sufficient to form heterochromatin and allow proper segregation. A reporter placed on the plasmid allows for the monitoring of establishment and maintenance of heterochromatin [81, 82]. The method commonly employed in our lab relies on the transient deletion of $\mathrm{clr}^{+}$. As previously stated, clr $4 \Delta$ causes a loss of all $\mathrm{H} 3 \mathrm{~K} 9 \mathrm{me}$ and therefore all heterochromatin [83]. The clr $4 \Delta$ can be combined with the deletion of other genes to test their role in the establishment of heterochromatin. In order to study de novo heterochromatin assembly, $\mathrm{clr}^{+}$is reintroduced back into its endogenous genomic locus in the double mutant cells and the ability of the cells to re-establish heterochromatin is measured by monitoring transcripts and $\mathrm{H} 3 \mathrm{~K} 9 \mathrm{me}[79,83,84]$. These methods allow for the identification of components necessary for proper heterochromatin establishment.

\section{RNAi Is Not Required for Establishment}

The first evidence that H3K9me is acting upstream of RNAi came from the observation that cells bearing deletions of RNAi components retain low levels of H3K9me [78]. More recently, a Tas 3 mutant known as tas $3 W G$ was created. This variant contains a mutation within the WG repeats of Tas 3 which interact with Ago1, disrupting the association of Chp1 and Tas3 with Ago1. This provided a unique way to separate TGS from PTGS. Although tas $3 W G$ has no defect in heterochromatin maintenance, when clr $4^{+}$or other Clr-C components are transiently deleted in this background centromeric heterochromatin cannot be established upon their reintroduction [79, 84]. These cells express high levels of pericentromeric transcripts, they fail to process the transcripts into siRNAs, and exhibit a loss of H3K9me accumulation. In contrast, when $d c r 1^{+}$or RDRC components, which retain low levels of $\mathrm{H} 3 \mathrm{~K} 9$ me when deleted, are transiently deleted in tas $3 W G$ cells, heterochromatin can be efficiently re-established and cells express siRNAs and accumulate H3K9me similarly to WT cells. Also, overexpression of Clr4 in RNAi defective $d c r 1 \Delta$ cells, in which residual $\mathrm{H} 3 \mathrm{~K} 9$ me had been removed through simultaneous deletion of $\mathrm{clr}^{+}$, promoted de novo deposition of $\mathrm{H} 3 \mathrm{~K} 9 \mathrm{me}$ [79]. Further, a Chp1 mutant in which the CD was mutated to reduce its affinity for H3K9me by around 10-fold was unable to establish heterochromatin after transient deletion of $\mathrm{clr}^{+}$, although 
siRNA levels were not impacted [85]. Together this data indicates that RNAiindependent deposition of $\mathrm{H} 3 \mathrm{~K} 9$ me by $\mathrm{Clr} 4$ is upstream of RNAi and RNAi serves to amplify the initial H3K9me signal at centromeres. Aspects of this mechanism are shared with other heterochromatic loci in fission yeast, where RNAi-dependent and independent mechanisms act redundantly in heterochromatin establishment and maintenance.

\section{Redundancy at Telomeres and Mating Type Locus}

In contrast to centromeres, it is known that the maintenance and establishment of heterochromatin at the mat locus involves both RNAi-dependent and RNAi-independent mechanisms. RNAi is required to establish heterochromatin, with the cenH element serving as a site for nucleation of heterochromatin [80, 86, 87]. Although RNAi is required for establishment, redundant mechanisms operate in heterochromatin maintenance at this locus. Heterochromatin can also be maintained at mat through two DNA binding factors, Atfl and Pcr1, which bind to DNA elements located between cenH and mat 3 that share homology with ATF/CREB DNA binding sites. These factors can then target Clr4, Swi6, and the HDACs Clr3 and Clr6 to the mating type locus in order to maintain heterochromatin at this locus [80, 88-90].

Like mat, there are redundant mechanisms of heterochromatin formation and maintenance at subtelomeric regions. The RNAi pathway can act to form heterochromatin at telomeres through $\mathrm{Clr}-\mathrm{C}$ recruitment at repetitive elements similar to the $d g$ and $d h$ repeats of the centromere [14]. Heterochromatin can also form at telomeres through an RNAi independent mechanism which involves Taz1, a protein that can bind to the telomeric repeats and is required for silencing of subtelomeric repeats [91, 92]. Taz1 binding allows for the recruitment of $\mathrm{Clr} 4$ to methylate $\mathrm{H} 3 \mathrm{~K}$ 9, allowing for Swi6 recruitment. These two mechanisms are thought to act redundantly in the establishment of heterochromatin at subtelomeric repeats $[22,92]$. HDACs also play a large role in silencing at telomeres due to the requirement for histone deacetylation prior to methylation [83].

\section{Requirements for RNAi Independent Establishment}

If the initial steps in the establishment of pericentromeric heterochromatin are independent of RNAi in fission yeast, what directs the de novo $\mathrm{H} 3 \mathrm{~K} 9$ me deposition? The minimum components required for heterochromatin establishment include both $\mathrm{Clr} 4$ and HDACs. Clr4 is critical for this process since it is the sole H3K9 HMT, and deacetylation of chromatin is required for both $\mathrm{Clr} 4$ recruitment and methylation of $\mathrm{H} 3 \mathrm{~K} 9$. In fission yeast, there are seven key HDACs: Hos2, Clr6, Clr3, Sir2, Hst2, and Hst4 (Table 1-1). 
Table 1-1. HDACs and their targets in $S$. pombe.

\begin{tabular}{llll}
\hline Class & Name & Target & Source \\
\hline Class I & Hos2 & Not identified & \\
& Clr6 & $\begin{array}{l}\text { Promoters } \\
\text { Coding regions } \\
\text { Broad specificity }\end{array}$ & 101 \\
Class II & Clr3 & H3K14 & 135 \\
Class III & Hst2 & Not identified & \\
(Sirtuins) & Hst4 & H3K56 & 105 \\
& Sir2 & H3K4 & $83,101,119$ \\
& & H3K9 & \\
& & H3K14 16 & \\
\hline
\end{tabular}




\section{Role of HDACs in Heterochromatin Formation}

HDACs were first identified in 1969 from calf thymus extracts [93]. Since their discovery, HDACs have been implicated in many biological processes, and much progress has been made in identification of both histone and non-histone substrates and mechanisms of HDAC regulation. One of the most studied roles of HDACs is the removal of acetyl groups from the $\epsilon$-amino group of lysines within histone tails, a mark deposited by HATs $[94,95]$. This is crucial in order to obtain the hypoacetylated state of heterochromatin and allow for $\mathrm{H} 3 \mathrm{~K} 9 \mathrm{me}$. There are three main classes of HDACs found in fission yeast, grouped based on sequence homology and domain structure: ClassI, ClassII, and Class III (Table 1-1).

Class I and II HDACs are considered classical HDACs and share a common mechanism of removing acetyl groups which relies on a set of conserved histidine, aspartic acid, and tyrosine residues within the HDAC core along with a zinc ion in order to hydrolyze the bond between the acetyl group and lysine residue [95, 96]. In fission yeast, Clr6 and Hos 2 fall into the Class I category. Clr6 has been shown to have broad specificity and has a genome wide role along with its role in mat silencing [22, 97]. Hos2 has been shown to have a role in preventing nucleosome loss along with promoting transcription by a mechanism not well understood $[97,98]$. Clr3 is a class II HDAC in fission yeast which is important for heterochromatin formation along with the class III HDAC Sir2, whose deacetylation mechanism differs from Classes I and II as discussed below [83, 97]. Hst2 and Hst4 also fall into the Class III category but their roles in chromatin structure regulation are not well understood $[99,100]$.

\section{Redundancy of Sir2 and Clr3 in Heterochromatin Maintenance}

Both Sir2 and Clr3 serve key functions in heterochromatin maintenance. While most studies of Sir2 and Clr3 have focused on telomeric and mat silencing, more recent work has shown that they are important for centromeric silencing. While deleting either Sir2 or Clr3 alone causes little defect in the maintenance of centromeric heterochromatin, deleting both simultaneously causes loss of centromeric heterochromatin, indicating that these HDACs act redundantly in centromeric heterochromatin maintenance [82, 83]. Deacetylation of $\mathrm{H} 3 \mathrm{~K} 9$ is catalyzed by Sir2, while Clr3 is the main HDAC responsible for deacetylation of histone H3 Lysine 14 (H3K14) [101]. For methylation of H3K9 by $\mathrm{Clr} 4$ to occur, both $\mathrm{H} 3 \mathrm{~K} 9$ and $\mathrm{H} 3 \mathrm{~K} 14$ must be deacetylated. Mutation of $\mathrm{H} 3 \mathrm{~K} 9$ and $\mathrm{H} 3 \mathrm{~K} 14$ to mimic acetylation results in the inability of overexpressed $\mathrm{Clr} 4$ to be recruited to the centromeric repeats [83]. While it is not entirely clear how H3K14 acetylation blocks Clr4 recruitment, deacetylation of this residue limits nucleosome gaps which can allow for RNA pol II access, possibly aiding in creating the proper environment necessary for Clr4 recruitment $[83,102]$. The current model proposes that deacetylation of both $\mathrm{H} 3 \mathrm{~K} 9$ and $\mathrm{H} 3 \mathrm{~K} 14$ is necessary for proper $\mathrm{Clr} 4$ recruitment and $\mathrm{H} 3 \mathrm{~K} 9$ methylation to maintain centromeric heterochromatin [83]. 


\section{Fission Yeast Sirtuins}

Hst2, Hst4, and Sir2 are all class III nicotinamide adenine dinucleotide (NAD $\left.{ }^{+}\right)$ dependent HDACs known as sirtuins. In contrast to classical HDACs which require a zinc cofactor, sirtuins require $\mathrm{NAD}^{+}$as a cofactor for removal of acetylation. This reaction releases nicotinamide, which is an inhibitor of sirtuins, and 2'-O-acetyl-ADPribose as a byproduct of the reaction [95, 103]. Hst 2 is not well characterized but has roles in silencing genes involved in membrane function and transport along with specific regions at the centromere [100]. Hst4 has been shown to have several roles including silencing genes involved in amino acid biosynthesis, retrotransposons, and silencing at the centromere and telomere $[100,104]$. Hst4 also has a role in deacetylating H3K56 during the DNA damage response for maintenance of genomic integrity [105]. Sir2 is the most studied of the sirtuins in fission yeast. It plays a significant role in heterochromatin formation at telomeres, mat, and centromeres [104].

\section{Structure of Sirtuins}

Sirtuins compromise a family of HDACs which are found in all organisms ranging from archaea to eukaryotes [106]. All sirtuins share a characteristic core domain which is highly conserved, ranging between 200 and 300 amino acids in size. This domain is commonly referred to as the HDAC domain and is responsible for the catalytic activity of the enzyme. Protein structures have shown that within the core there is a conserved Rossman fold domain which binds to $\mathrm{NAD}^{+}$, and a zinc binding domain which serves a structural role. The junction of these two domains forms the catalytic site of the sirtuin HDAC core [107]. In addition to the catalytic domain, some Sirtuins also contain long N-terminal (NTD) and C-terminal domains (CTD). These domains are highly variable and tend to contain minimal structural elements. Although the functions of these domains are still unclear, research suggests they regulate HDAC activity or complex formation $[108,109]$.

\section{Sir2 Homologs}

In budding yeast, there are five sirtuins, Sir $2 p$ and Hst1p-Hst4p, with Sir $2 p$ being the first identified sirtuin [110]. Budding yeast Sir2p is the homolog of fission yeast Sir2. Although budding yeast heterochromatin lacks H3K9me and HP1 coating, it is characterized by hypoacetylation, and Sir $2 p$ is required for silencing at telomeres and the mating type region. Sir $2 p$ is recruited to these regions by Sir $3 p$ and Sir $4 p$, non-HDAC proteins which interact with DNA binding factors, forming the SIR complex. This allows for deacetylation of lysine 16 of histone H4 (H4K16), the prime target of Sir2p, creating

a deacetylated environment favorable for recruiting more SIR complex and spreading heterochromatin $[111,112]$.

In humans, there are seven Sirtuins (SIRT 1-7) with SIRT1 being the closest homolog to fission yeast Sir2 $[83,113]$. Human sirtuins have been implicated in 
numerous processes including regulation of transcription factors, metabolism, and the tumor suppressor p53, and they are overexpressed in several types of cancer [110, 113116]. SIRT1 deacetylates H3K9 and H4K16 for constitutive heterochromatin maintenance at the centromere, but the importance of centromeric heterochromatin in mammals is unclear [72, 117]. Interestingly, SIRT1 can also regulate the SUV39H1 HMT, an enzyme which catalyzes $\mathrm{H} 3 \mathrm{~K} 9 \mathrm{me} 3$ at mammalian centromeres, by deacetylation of lysine within the catalytic core, upregulating HMT activity [118]. Sirtuins thus play important conserved roles in heterochromatin assembly and fission yeast provide a useful tool for dissection of their functional roles.

\section{Targets of Sir2}

Early in vitro work in fission yeast using $\mathrm{NAD}^{+}$hydrolysis as a measure of deacetylation by recombinant Sir2 identified H3K9 and H4K16 as targets of Sir2 [119]. Recent work suggests that along with deacetylation of H3K9 and H4K16, Sir2 can also deacetylate H3K4 and has some ability to deacetylate H3K14 [83]. ChIP assays show that $\mathrm{H} 3 \mathrm{~K} 9 \mathrm{ac}$ is the prime target of Sir2 in the process of heterochromatin formation in vivo [97, 119]. In addition, high resolution quantitative mass spectrometry histones sir $2 \Delta$ fission yeast has indicated that deletion of Sir2 does not cause global changes in histone $\mathrm{H} 3$ and $\mathrm{H} 4$ acetylation, though an increase in peptides containing acetylation at $\mathrm{H} 3 \mathrm{~K} 9$, 14, 18, and 23 were detected. No significant changes in histone $\mathrm{H} 4$ acetylation were observed in a sir $2 \Delta$ strain of fission yeast [120]. These works show that Sir2 can deacetylate several residues key to the formation of centromeric heterochromatin in fission yeast.

\section{Sir2 Is Required for Centromeric Heterochromatin Establishment}

The role of fission yeast Sir2 has mainly focused on its role in mating type and telomeric heterochromatin formation. Sir2 is located primary in the nucleus, with ChIP experiments providing some evidence of enrichment of Sir2 at centromeres, telomeres and the mating type locus, although only low levels of enrichment were observed [104]. At the mating type locus, sir $2 \Delta$ cells exhibit loss of silencing of a reporter gene along with increased $\mathrm{H} 3 \mathrm{~K} 9 \mathrm{ac}$ and $\mathrm{H} 3 \mathrm{~K} 14 \mathrm{ac}$ and reduced Swi6 association with chromatin although $\mathrm{H} 3 \mathrm{~K} 9$ me was not examined. At the subtelomeric repeats, similar phenotypes were observed in sir $2 \Delta$ cells consistent with defective heterochromatin formation, and the HDAC activity of Sir2 was shown to be required for silencing [83, 104, 119]. Mutation of a conserved asparagine, residue 247 to alanine within the nucleotide binding pocket of the Sir2 HDAC core (Sir2 N247A) caused loss of telomeric silencing, with loss of H3K9me, increased H3K9ac, and loss of Swi6 localization in vivo [83, 121, 122]. In vitro, Sir2 N247A displays no HDAC activity [83]. At centromeres, the role of Sir2 was less pronounced, affecting mostly the $i m r$ repeats but having only minor effects on the otr repeats and no effect on the cht region [104, 119]. Loss of Sir2 causes a loss of the distinctive pattern of Swi6 foci in the cell as visualized by fluorescent microscopy, although unlike many mutants disrupting Swi6 localization, no chromosome segregation 
defects were observed [104]. It is possible that enough Swi6 remains at the pericentromeric repeats to ensure proper chromosome segregation since sir $2 \Delta$ only slightly impacts the otr [104].

The role of Sir2 at the centromere has seen renewed focus in recent years. While Sir2 is not required to maintain heterochromatin at the centromere, it is required to establish pericentromeric heterochromatin $[82,83]$. This is consistent with $\mathrm{H} 3 \mathrm{~K} 9$ as a key target of Sir2 deacetylase activity. In agreement with earlier work, sir $2 \Delta$ and Sir2 N247A have little impact on centromeric heterochromatin maintenance, with cells retaining levels of H3K9me similar to WT strains and no reduction in Swi6 association. In contrast, Sir2 is required for centromeric heterochromatin establishment since reintegration of $\operatorname{clr} 4^{+}$into sir $2 \Delta \operatorname{clr} 4 \Delta$ strains does not re-establish silencing, H3K9me accumulation, or binding of RNAi components to the centromere, indicating that Sir2 is required to establish heterochromatin de novo and for the initial deposition of $\mathrm{H} 3 \mathrm{~K} 9 \mathrm{me}$ [83]. This same requirement for Sir2 in establishment was also observed using a minichromosome to monitor establishment [82]. As previously discussed, H3K9me can be deposited de novo in the absence of RNAi since low levels of H3K9me accumulate at centromeres in $\operatorname{clr} 4 \Delta \mathrm{dcr} \Delta$ strains upon $\operatorname{clr} 4^{+}$overexpression [79]. However, when sir $2^{+}$ is further deleted from a $d c r 1 \Delta \operatorname{clr} 4 \Delta$ strain and $\operatorname{clr} 4^{+}$is overexpressed in these cells, no $\mathrm{H} 3 \mathrm{~K} 9 \mathrm{me}$ accumulates at centromeres [83]. Taken together, this research indicates the main function of Sir2 at the centromere is in the establishment of heterochromatin. It also indicates that Sir2 is necessary for the de novo establishment of H3K9me in RNAi deficient backgrounds.

Although sir $2 \Delta$ causes a complete loss of establishment, the conclusion was less clear when the Sir2 N247A HDAC mutant was tested for its ability to establish pericentromeric heterochromatin. Sir2 N247A shows a limited ability to establish heterochromatin de novo. After $\mathrm{clr} 4^{+}$reintegration, otr transcript levels are lower than those of a $\operatorname{cl} 4 \Delta$ but not back to WT levels, suggesting that some ability to establish heterochromatin is retained in the sir $2 N 247 A$ background [83]. This raises an interesting possibility that Sir2 may have functions independent of its HDAC activity.

\section{Roles of Sirtuin Auxiliary Domains}

Little is known about domains of Sir2 outside the HDAC domain or about its recruitment to areas of heterochromatin in fission yeast. Sir2, like its homologs, has long NTD and CTD. Although the HDAC core is conserved in sequence and structure among Sir2 homologs, little conservation is observed within the NTD and CTD which appear mainly unstructured. It is possible these domains serve as regulatory elements or protein interaction surfaces based on their function in homologs of Sir2.

The NTD and CTD of mammalian SIRT1 have been shown to undergo extensive PTM. SIRT1 is phosphorylated at multiple sites within the NTD and CTD, with phosphorylation having roles in regulating HDAC activity, cell cycle progression, and cell survival [123-127]. Phosphorylation of the NTD and CTD by CyclinB/Cdk1, a $\mathrm{G}_{2} / \mathrm{M}$ 
phase cyclin-dependent checkpoint kinase, leads to increase HDAC activity and allows for proper entry into $\mathrm{G}_{2}$ phase [123]. The serine/threonine kinase CK2 has been shown to phosphorylate the CTD of SIRT1 and can increase HDAC activity, leading to increased cell survival $[125,128]$. Phosphorylation of SIRT1 by the c-Jun N-terminal kinase 1 and 2 (JNK1 and JNK2), mitogen activated protein kinases, within the NTD leads to increased nuclear localization, H3 deacetylation, and protein stabilization $[124,126]$. It has also been shown that sumoylation of the CTD of SIRT1 in human cells leads to increased HDAC activity while Sir2p NTD sumoylation in budding yeast impairs the Sir2p/Sir4p interaction, leading to defects in telomeric silencing $[129,130]$. Although PTM of the Sir2 NTD and CTD has not been studied in fission yeast, it is likely that these regions play roles in regulation or complex formation and their study might help explain why the HDAC defective Sir2 N247A mutant can establish limited de novo heterochromatin while cells lacking Sir2 cannot.

While most HDACs act in complexes which aid in their targeting to specific sites, such as Sir3p and Sir4p facilitating targeting of Sir2 in budding yeast, no Sir2-containing complex has been identified in fission yeast. This leads to important questions about ancillary proteins that may influence targeting to Sir2 in fission yeast, which domains of Sir2 are important for these interactions, and whether PTMs on Sir2 influence targeting [131-133].

\section{Aims to Understand the Role of Sir2 in Heterochromatin Assembly}

Although it is clear Sir2 is a key factor in the establishment of heterochromatin, the details of how this works still require clarification. Clr4 and Sir2 are required for the initial de novo deposition of H3K9me which can occur independently of RNAi [79]. To date, no characterization of Sir2 other than the HDAC domain has been performed, so the function of these domains remains unclear. Since the HDAC defective Sir2 N247A mutant exhibits some ability to establish pericentromeric heterochromatin, it is possible that the role of Sir2 in this process extends beyond just HDAC activity and includes both the NTD and CTD [83]. Also, how Sir2 is recruited to areas of heterochromatin formation is unknown. Here we sought to examine how Sir2 is recruited to chromatin and determine if the NTD and CTD are important for Sir2 activity, possibly independent of HDAC activity. We believe that a better understanding of the answers to these questions will aid in understanding how heterochromatin is formed.

\section{Role of Clr3 in Heterochromatin Assembly}

The class II HDAC Clr3, a member of the Snf2/HDAC-containing repressor complex (SHREC), acts redundantly with Sir2 in the maintenance of pericentromeric heterochromatin but importantly, like Sir2 is necessary for the establishment of de novo pericentromeric heterochromatin [unpublished communication from Janet Partridge on 03/10/2017]. Clr3 was first identified in a genetic screen for mutants with disrupted mat silencing where it is required for proper silencing [35]. Deletion of $\operatorname{clr} 3^{+}$has a partial 
defect in silencing at the imr and otr repeats of the centromere and subtelomeric heterochromatin with no observed chromosome segregation defects $[21,134,135]$. Clr3 was first described as a probable HDAC due to sequence similarities between budding yeast Hda1p and mammalian HDAC1 [90]. In vitro data suggests the major target of Clr3 is $\mathrm{H} 3 \mathrm{~K} 14 \mathrm{ac}$ along with some deacetylase activity towards H4K16ac [135]. This was confirmed by in vivo work using quantitative liquid chromatography-tandem mass spectrometry (LC-MS/MS), showing Clr3 primarily targets $\mathrm{H} 3 \mathrm{~K} 14$ ac on histones with $\mathrm{H} 3 \mathrm{~K} 9 \mathrm{me}$, indicating it primarily operates at heterochromatic regions [120].

\section{Similarities between NuRD and SHREC}

In the late 1990's, a unique complex containing two distinct enzymatic activities, chromatin remodeling activity and HDAC activity, was identified [136-138]. The complex, termed the nucleosome remodeling and deacetylase complex (NuRD), contains around ten subunits and has been purified from various systems ranging from flies to humans (Figure 1-3). The chromodomain-helicase DNA binding protein 3 and 4 (CHD3/4), also known as Mi-2 $\alpha$ and $\beta$, are ATPases which contain two plant homeodomains (PHD) fingers important for binding histones and two atypical CDs which bind to DNA $[139,140]$. These enzymes contribute the chromatin remodeling function of NuRD. Histone deacetylase 1 and 2 (HDAC1/2) are Class I HDACs responsible for the HDAC activity. Along with the enzymatic core, several other proteins lacking enzymatic activity can associate with NuRD. These include the methyl-CpGbinding domain proteins 2 and 3 (MBD2/3), of which only MBD2 has the ability to bind methylated DNA [139]. Metastasis-associated gene 1, 2, and 3 (MTA1/2/3) contain domains important for interacting with other proteins. Retinoblastoma binding proteins 4 and 7 (RBBP4/7) contain domains important for binding histone H4. The GATA zinc finger domain containing proteins $2 \mathrm{~A}$ and $2 \mathrm{~B}(\mathrm{GATAD} 2 \mathrm{~A} / 2 \mathrm{~B})$ transcriptional repressors have also been shown to complex with NuRD and can interact with histones [139, 141, 142]. The primary and most studied role of NuRD involves its role in transcriptional regulation of genes although it has also been shown to be involved in genomic stability and DNA damage response. Deregulation of NuRD subunits have also been implicated in several types of cancer $[139,143]$.

The fission yeast SHREC complex shares similarity to the NuRD complex, having both HDAC and chromatin remodeling activities within the same complex, along with non-enzymatic subunits which have domains similar to subunits of NuRD (Figure 1-3) [144, 145]. SHREC consists of Clr1, Clr2, Chp2, and Mit1 along with Clr3 [48, 144, 146, 147]. Clr1 and Clr2 were identified along with Clr3 for their requirement in mat silencing [35, 49, 148]. The function of Clr1 and Clr2 is not entirely certain although recent work has provided insight into their function. Clr1 serves as a scaffold for the assembly of SHREC and Clr2 has a domain similar to the MBD domains of MBD2/3 which recognize methylated DNA. Although methylated DNA is not found in fission yeast, it was shown this domain can bind nucleic acid and is important for the function of SHREC at heterochromatic regions [149]. Chp2 contains a CD and is important for recruiting SHREC to heterochromatic regions to deacetylate H3K14 [48]. Mit1 is the 

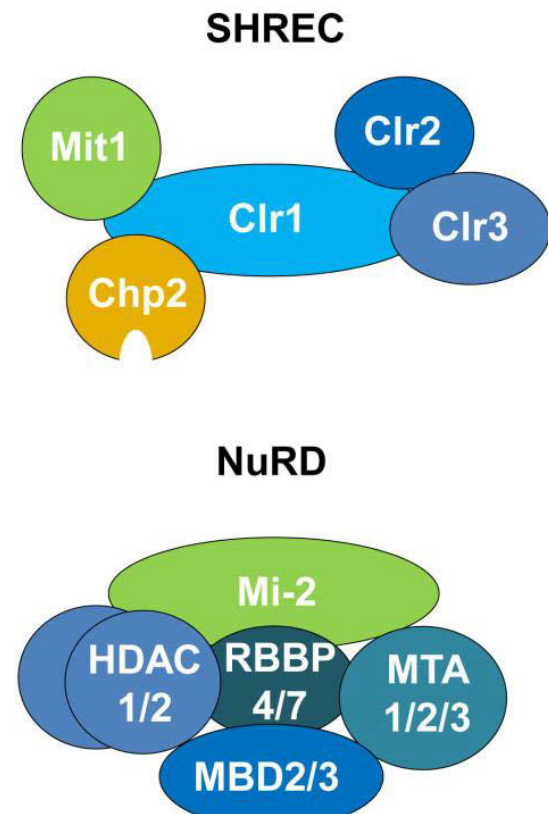

Figure 1-3. Comparison of the SHREC and NuRD complexes.

A. Schematic showing the architecture of SHREC as proposed by biochemical and structural studies. SHREC is composed of the ATP-dependent chromatin remodeler Mit1, the HDAC Clr3, the CD-containing protein Chp2, Clr2, and the scaffold protein Clr1. B. Schematic showing the proposed architecture of the NuRD complex. The core components of NuRD consist of the ATP-dependent chromatin remodeler Mi-2, HDACs1 and 2, the Methy-CpG-binding proteins MBD2 and 3, the Metastasis associated proteins 1, 2, and 3, and the histone binding proteins RBBP4 and 7. 
Snf2-related ATP-dependent nucleosome remodeler which acts on heterochromatic regions, providing chromatin remodeling activity similar to Mi-2 of NuRD. It contains a PHD domain thought to bind to histone $\mathrm{H} 3$ and a CD which can bind DNA. These domains are required for function and likely aid in the recruitment of SHREC to heterochromatic regions [145]. Due to the role of NuRD in a variety of processes, it is crucial to understand the basic biology of the complex. The fission yeast SHREC complex provides an opportunity to study the basic roles of a similar complex in a simple model system.

\section{Role of SHREC at Heterochromatic Loci}

Like NuRD, SHREC is important for gene regulation. All components of SHREC are required for its role in heterochromatin maintenance at centromeres, mat, and subtelomeres [144]. At these regions, Clr3 of SHREC deacetylates H3K14 to allow for silencing of these regions. Mit1 acts mainly at heterochromatic loci where it can mobilize nucleosomes onto regions which are refractory to nucleosome occupancy $[102,145]$. Interestingly, deacetylation of H3K14 does not appear to be the sole function of Clr3. It has been shown that deletion of $\mathrm{Clr} 3$ has a more severe impact on nucleosome occupancy in comparison to strains which prevent deposition of $\mathrm{H} 3 \mathrm{~K} 14 \mathrm{ac}$ through deletion of the responsible HATs. This indicates $\mathrm{Clr} 3$ may serve additional roles along with the deacetylation of H3K14 [145]. Clr3 has been implicated in suppressing histone turnover at mat and pericentromeric regions, preventing the dilution of heterochromatic histone PTMs and promoting the stable inheritance of heterochromatin [150]. It has previously been shown that deletion of $\mathrm{Clr} 3$ can impact nucleosome occupancy at regions unaffected by Mit1 deletion, especially at pericentromeric regions [102]. The difference in phenotype between mutants in different SHREC subunits raises the possibility of a distinct role for different members of the complex, possibly acting through subcomplexes.

If $\mathrm{Clr} 3$ can act in a manner independent from SHREC, it raises the question of how this action is occurring. One possibility is that Clr3 has additional targets beyond $\mathrm{H} 3 \mathrm{~K} 14 \mathrm{ac}$. Until recently, there was little information on the role of Clr3 in vivo. With the ability to look at PTMs using mass spectrometry, there is renewed interest in examining the effects of deletion of HDACs on global histone PTMs. Although recent work has looked at the targets of $\mathrm{Clr} 3$ on $\mathrm{H} 3$ and $\mathrm{H} 4$, the effects on $\mathrm{H} 2 \mathrm{~A}$ and $\mathrm{H} 2 \mathrm{~B}$ PTMs have not been characterized [120]. In support of this hypothesis, it was discovered that the Neurospora crassa Clr3 homolog, HDA1, deacetylates H2B at several lysines on its NTD but the role of these PTMs is not well understood [151]. Histone H2B K5, 6, and 10 are conserved in fission yeast and can be acetylated although the HATs which target these residues remain unknown (Figure 1-4) [152]. In budding yeast, acetylation of lysines corresponding to $\mathrm{K} 5,10$, and 15 along with several other lysines within the H2B NTD can lead to transcriptional activation of specific genes involved in metabolism [153]. It is possible that PTM of the H2B NTD in fission yeast may be playing a part in the role of Clr3 at sites of heterochromatin. 
S. pombe

N. crassa

S. cerevisiae HTB1

S. cerevisiae HTB2

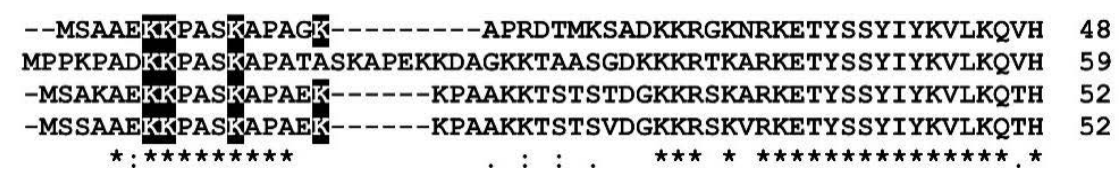

Figure 1-4. Conservation of the H2B NTD.

Alignment showing conservation of the H2B NTD between $S$. pombe, N. crassa, and $S$. cerevisiae. The NTD of H2B contains lysine residues conserved between different Fungai. 


\section{Aims to Understand the Role of Clr3 in Heterochromatin Assembly}

It is essential to understand the basic biological role of the HDAC Clr3 in order to better understand homologs important in higher eukaryotic systems. Although the role of Clr3 in H3K14 deacetylation has been extensively studied, roles outside of this function

remain unclear. Since some evidence suggests that SHREC can act as distinct sub-entities as well as in the complete SHREC complex, we sought to elucidate whether these subcomplexes exist. We also sought to determine whether Clr3 can target sites of acetylation in the NTD of H2B and whether these PTMs influence heterochromatin assembly in fission yeast.

\section{Fission Yeast as a Tool to Study Cancer Associated Mutations of DDX3X}

Recently, the ATP dependent RNA helicase DDX3X, named for its location on the $\mathrm{X}$ chromosome, was found to be a target of mutation in medulloblastoma (Figure 1-5A) [154-156]. Currently, little information is available regarding the potential biological impacts of these mutations and their contribution to cancer. In order to quickly access the basic biological significance of cancer-associated DDX3X mutations, we turned to fission yeast. The fission yeast homolog of DDX3X is Ded1, whose core domains and indeed the residues mutated in medulloblastoma are highly conserved with DDX3X (Figure 1-5B) [157]. Due to the high degree of conservation, along with quick generation time and ease of genetic manipulation, fission yeast provide an excellent tool to gain information about the role of DDX3X mutations which can then be applied to studies in higher eukaryotes.

\section{DEAD-Box Helicases}

Human DDX3X and fission yeast Ded1 belong to the DEAD-box family of RNA helicases which utilize ATP hydrolysis in the process of remodeling nucleic acid secondary structure [158]. This family was first described in the late 1980's based on the discovery of several bacterial helicases which shared homology with the eukaryotic initiation factor eIF-4A, another DEAD-box RNA helicase $[159,160]$. Since then, members of the DEAD-box family have been found in species ranging from viruses to metazoans $[158,161]$. Members of this family have key roles in almost all processes involved in RNA metabolism. For example, they are involved in transcription, where they can physically interact with transcription factors, RNA transport, aiding in the transport of mRNA from the nucleus, and translation of genes with complex untranslated regions (UTRs) [162-164]. DEAD-box helicases have become an area of intense research due to emerging evidence of their roles in disease. 
Figure 1-5. Domains and cancer associated mutants of Human DDX3X. A. Location of cancer associated mutants within the core domains of DDX3X. DDX3X consists of a helicase core made up by two recA-like domains shaded in light blue (Domain 1) and orange (Domain 2). All medulloblastoma associated DDX3X mutants, along with the R351W DDX3X mutant found in infant acute lymphoblastic leukemia, discovered by the PCGP cluster within Domains 1 and 2. An ATP binding loop (shaded in purple) is necessary for ATP hydrolysis by DDX3X. DDX3X also has an NTD (shaded in blue) and a CTD (Shaded in red) whose functions are unclear. B. Conservation of motifs between DDX3X homologues in S. cerevisiae, S. pombe, and D. melanogaster, and eIF4A from $S$. cerevisiae. Key domains of DDX3X along with the ATP binding loop are conserved between the homologs, including fission yeast Ded1. Cancer-associated mutations found in DDX3X are shaded in purple with conserved residues highlighted in blue. The ATP binding loop of DDX3X and its homologs contains a highly-conserved phenylalanine which is important for its function.

Source: Reprinted with permission from Elsevier Inc. Figure designed by Eric Enemark (SJCRH). Epling, L.B., C.R. Grace, B.R. Lowe, J.F. Partridge, and E.J. Enemark, Cancer-associated mutants of RNA helicase DDX3X are defective in RNA-stimulated ATP hydrolysis. J Mol Biol, 2015. 427(9): p. 1779-96. Creative Commons license: https://creativecommons.org/licenses/by/4.0/. DOI: 10.1016/j.jmb.2015.02.015. 
A.

DDX3X

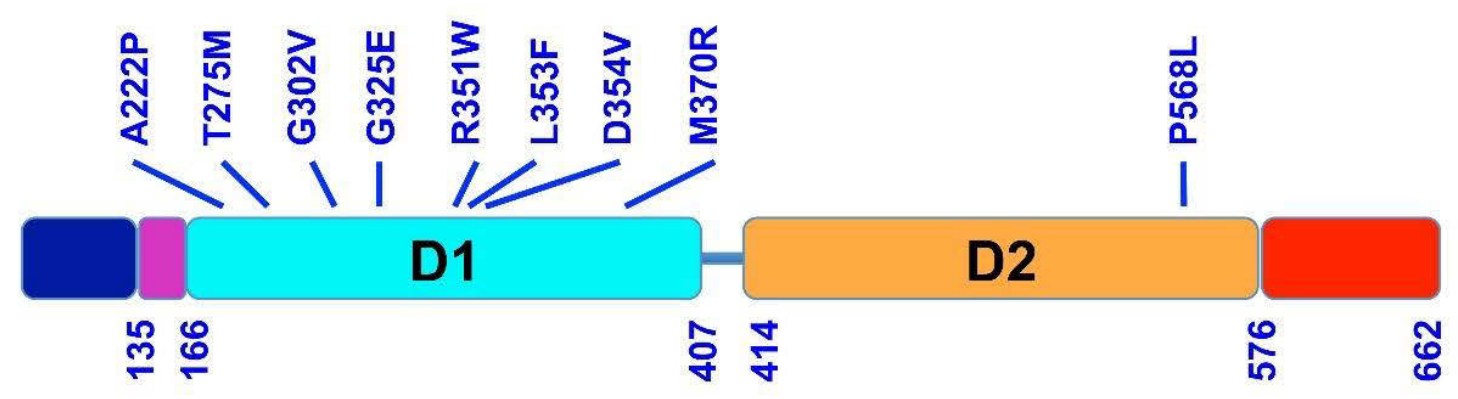

B.
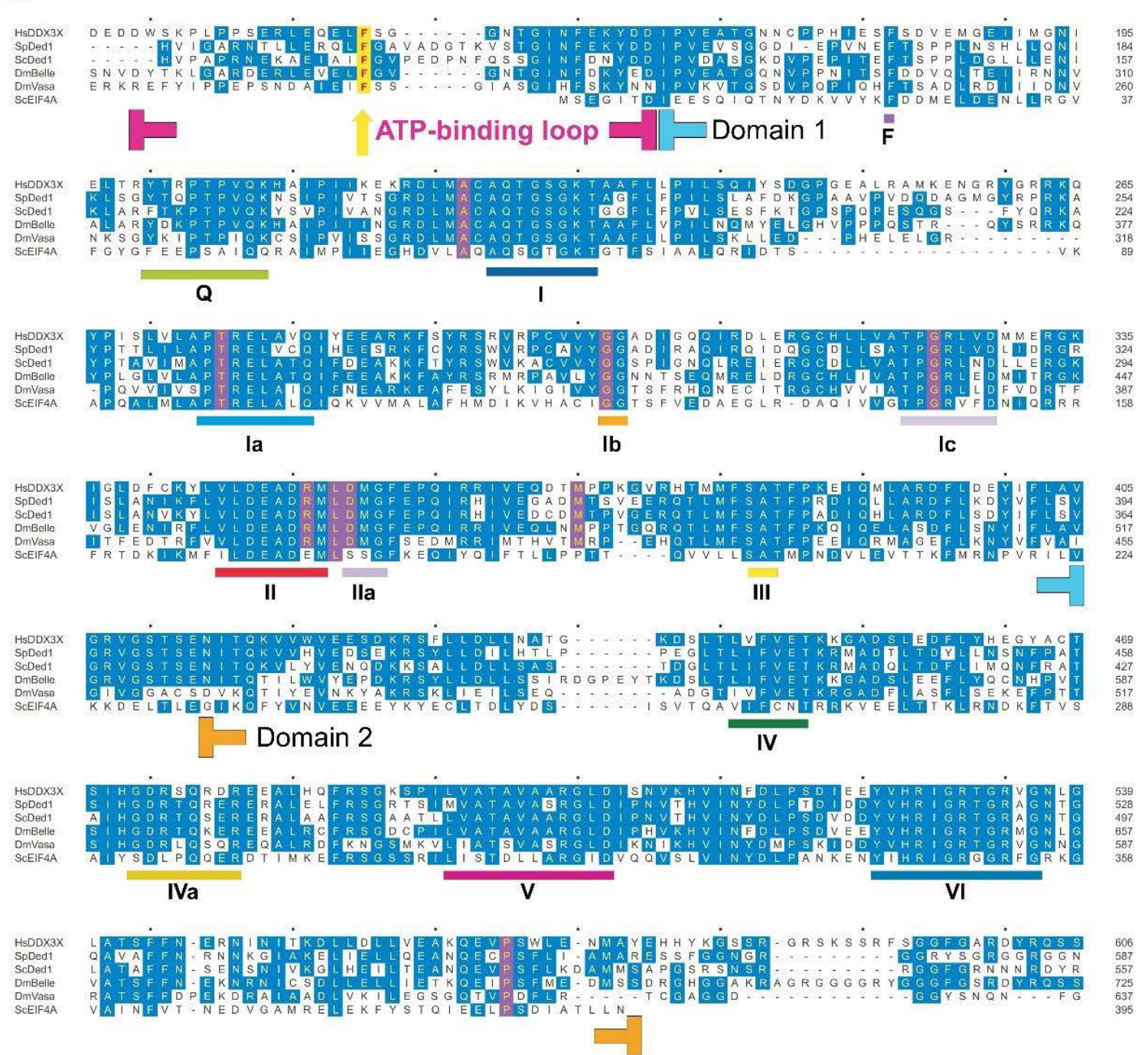


\section{Classification, Structure, and Mechanism of Action}

DEAD box helicases belong to helicase superfamily 2 (SF2), the largest and most variable superfamily, defined by a core of two highly conserved "Rec-A like" helicase domains separated by a flexible linker [158]. These conserved helicase domains contain at least eight characteristic sequence motifs common to SF2 family members $[158,160$, 165, 166]. SF2 members also have highly variable NTDs and CTDs which may function in helicase localization, regulation, or substrate recognition [164, 167]. Specifically, the DEAD-box family, which makes up the largest subgroup of SF2, share twelve conserved sequence motifs within the helicase domains (Figure 1-5B). Motifs Q, I (Walker A motif), II (Walker B motif), and VI are involved in ATP binding and hydrolysis, with the DEAD-box name being derived from the Walker B motif sequence "Asp-Glu-Ala-Asp" conserved among the subfamily $[157-160,163,164,168]$. DDX3X also contains an Nterminal extension known as the ATP binding loop (ABL) which interacts with ATP and may serve to stimulate ATP hydrolysis after binding certain substrates [157]. Binding to the nucleic acid substrate involves motifs Ia, Ib, Ic, IV, IVa and IVb. Motifs III and V are involved in the coordination between ATP binding and nucleic acid substrate binding $[158,161,163,169]$. These domains and the motifs contained within them are crucial for DEAD-box helicases to perform their unwinding activities on their substrate.

DEAD-box helicases tend to be non-processive in contrast to classical helicases, performing their manipulation on small segments and dissociating from their target nucleic acid rather than translocating down the target $[165,166]$. They perform their manipulation of nucleic acid through inter-domain movements of the two helicase domains within the core of the protein $[158,168]$. These helicases have been implicated in manipulating ssRNA with secondary structure, dsRNA, and RNA/DNA hybrids [165]. Although the biological substrates of DDX3X are unknown, in vitro it has been shown to act on specific RNA structures, being stimulated by ssRNA/dsRNA hybrids but not ssRNA or dsRNA alone suggesting a preference for particular substrates $[157,165]$. Recent work has shed light onto the mechanism by which DEAD-box RNA helicases unwind their nucleic acid substrate. Unlike many helicases which utilize NTP hydrolysis to drive unwinding activity, DEAD-box helicases have been shown to only require ATP binding to drive unwinding activity [170]. Studies into the DEAD-box helicase Mss116p suggest that the binding of ATP and the target RNA duplex is sufficient to promote the closure of the core formed by the two helicase domains leading to unwinding of the substrate by strand separation. Hydrolysis of the ATP serves to open the core allowing for further binding and substrate processing [170].

\section{Mutation of DDX3X in Medulloblastoma}

DDX3X has been implicated to have roles in many processes of RNA metabolism including transcription, translation, RNA storage, nuclear export of messenger RNA (mRNA), pre-mRNA splicing, and ribosome biogenesis [157, 164, 171-181]. Since DDX3X is involved in so many different processes key to proper cellular function, it would be reasonable to hypothesize that mutations affecting its function would contribute 
to disease. Recently, mutations or translocations within DDX3X have been implicated in various cancer types ranging from several types of leukemia to medulloblastoma [154$157,178,180,181]$. Due to its apparent role in disease, DDX3X has become a target of intense interest in cancer research.

The finding of DDX3X mutations in medulloblastoma has opened a new window into the molecular forces driving certain medulloblastoma tumors. Medulloblastoma is cerebellar primitive neuroectodermal in origin and although rare in adults, medulloblastoma is the most common type of malignant brain tumor in children comprising around $20 \%$ of all central nervous system tumors affecting children under the age of 14 [182-186]. Since brain tumors account for the majority of cancer related mortality in children with over 4000 new cases each year, much research is being performed to develop better treatment options for pediatric brain tumors [185]. Currently, the overall survival rate of medulloblastoma is around $60-70 \%$ for high risk groups and nearly $80 \%$ for average risk groups but the need for more targeted therapies is great since a high percentage of surviving patients experience long term negative neurological side effects $[184,187,188]$.

\section{Medulloblastoma Subgroups}

It is now commonly recognized that there are four main subgroups of medulloblastoma: WNT, SHH, Group 3, and Group 4. These subgroups are based on transcriptional profiling and tumor markers and were assigned to provide more targeted therapy to different medulloblastoma tumors $[182,189,190]$. The WNT subgroup derives its name from over-activation of the WNT pathway along with frequent mutation of CTNNB1, the gene encoding $\beta$-catenin 1 . Although this tends to be the rarest subgroup accounting for less than $15 \%$ of all medulloblastoma cases, it has the best prognosis with a survival rate of greater than $90 \%[184,189,190]$. The SHH subgroup is characterized by frequent mutations in PTCH, SMO, and SUFU, all components of the sonic hedgehog signaling pathway which lead to pathway over-activation. This group also tends to have high levels of MYCN expression. The SHH subgroup accounts for nearly $25 \%$ of medulloblastoma cases and has a survival rate of 60-80\% [184]. The Group 3 subgroup accounts for nearly $28 \%$ of medulloblastoma cases and is often but not exclusively characterized by MYC amplification and immunohistochemical expression of natriuretic peptide receptor 3. Cases falling into this subgroup tend to be metastatic and have the lowest prognosis of all the subgroups with a survival rate lower than $50 \%[182,184,188$ 190]. Subgroup 4 represents the most common subgroup, consisting of more than $30 \%$ of all cases. The molecular pathogenesis of this subgroup is not as well understood as others, but isochromosome $17 \mathrm{q}$ is observed in over $60 \%$ of cases. The survival rate is fairly good, being between $60-80 \%$ [184, 188-190]. Although much research has been done into the different subgroups, unfortunately the same aggressive treatment regimen of surgery, radiation, and chemotherapy is normally administered to treat each subgroup, leading to overtreatment of groups with better prognosis while undertreating subgroup 3 which has the worst prognosis [154, 188-190]. Though the survival rate for medulloblastoma is relatively good, developing more targeted treatments for each 
subgroup is essential since overtreatment can lead to negative life-long neurological consequences which compromise a patient's quality of life [189].

Recent studies have shed light on the genomic landscape of pediatric medulloblastoma through the use of whole genome sequencing (WGS) [154-156]. DDX $3 \mathrm{X}$ was found to be mutated in around $10 \%$ of pediatric medulloblastoma tumors, indicating that mutation of DDX3X may have a role in the molecular dysfunction of certain tumor types [178]. Mutations of DDX3X in medulloblastoma were mainly found in the WNT subgroup, with nearly $50 \%$ of pediatric cases containing a mutated form of DDX3X. DDX3X mutations were also found in the SHH subgroup but at a dramatically lower frequency of around 11\% [191]. The mutations of DDX3X mainly clustered to the two helicase domains of DDX3X. Of particular interest to us were the mutations found by the St. Jude Pediatric Cancer Genome Project (PCGP), which identified eight novel somatic missense mutations within the helicase domains of DDX3X from WGS of pediatric medulloblastoma tumors along with one DDX3X mutation found in infant acute lymphoblastic leukemia (Figure 1-5A, B) [154, 157]. Domain 1 was found to have mutations of: A222P, T275M, G302V, G325E, L353F, D354V, and M370R while P568L was found in domain 2 [154]. Three of the mutations, T275M, G302V, and G325E, lie within helicase motifs important for binding of RNA and are implicated in interacting with RNA when overlaid with the available structure of the Drosophila DDX3X homolog Vasa [157]. A222P also lies near another RNA binding motif, motif I. R351W, a mutation found in infant acute lymphoblastic leukemia, lies at the end of the Walker B motif, which binds ATP [157]. L353F along with D354V lie next to or within motif IIa respectively, both motifs involved in binding to RNA. These residues also lie close to the Walker B ATP binding motif. P568L was the only mutation from the PCGP project to be found in Domain 2 and does not lie near any identified motifs but the loss of the proline at this position may impact the secondary structure of Domain $2[156,157]$. Due to the location of these mutations, it can be inferred that several of them likely impact either the nucleic acid binding ability or ATP binding/hydrolysis ability of DDX3X [157]. Examination of the biological implication of these mutations will prove invaluable in order to develop better, more targeted treatments for certain types of medulloblastoma and decrease negative life-long side effects associated with the current treatments.

\section{Role of DDX3X and Homologs in Translation}

The biological implication of DDX3X mutations in medulloblastoma is not currently well understood, although several new studies have emerged including our own, suggesting that mutation of DDX3X impacts its role in translation of transcripts with complex UTRs. Evidence of such a role began to emerge in the early 1990's when it was shown that the $S$. cerevisiae homolog of DDX3X, Ded1p, is likely involved in translation of mRNA both in vitro and in vivo [192]. The fission yeast Ded1 is encoded by an essential gene and shares a high degree of homology of key domains with human DDX3X [157]. It was shown that Ded1 plays an essential role in translation using a thermosensitive mutant of Ded1. Although inactivation of Ded1 leads to a defect in general translation, the translation of mRNAs with long complex UTRs were more 
negatively impacted [162]. Consistent with this data, DDX3X was implicated in facilitating the translation of cyclin E1 [174]. More recent evidence has shown that DDX3X is involved in binding to and translation of mRNAs containing TAR elements (trans-activation response element) and can interact directly with eIF4E and eIF4G in translation initiation $[177,193]$. Much research into the role that mutation of DDX3X plays in medulloblastoma is now focused on the role of DDX3X in translation.

\section{Aims for Examination of DDX3X Mutations}

Since the role of DDX3X in translation appears to be conserved with the fission yeast Ded1, fission yeast provides an excellent tool to examine the effects of DDX3X

mutations in order to elucidate the biological consequences of DDX3X mutations found in medulloblastoma. Although Ded1 function is essential for fission yeast, thermosensitive mutants of Ded1 provide the ideal tool to quickly address the consequences of specific mutations of DDX3X in order to begin to provide insight into processes affected by these mutations. This data can be used to further study these mutations in order to develop more targeted treatments for specific subgroups of medulloblastoma with less long-term negative side effects. 


\section{CHAPTER 2. METHODOLOGY}

\section{Strain Construction}

All yeast strains used in this study are listed in Table A-1. The creation of the $\operatorname{sir} 2 \Delta$, $\operatorname{sir} 2 \Delta \operatorname{clr} 3 \Delta$, and $\operatorname{sir} 2 \Delta^{E}$ establishment strains was previously described [83]. To study the role of Sir2 in heterochromatin maintenance and establishment, these strains were transformed with episomal pREP81-Sir2-3x V5-LEU2 ${ }^{+}$constructs and maintained on media lacking leucine.

To create the integrated sir2-3x V5 constructs, the ORF of Sir2 was replaced with $\mathrm{ura}^{+}$marker gene. The $\mathrm{ura}^{+}$marker was then replaced by $\operatorname{sir} 2-3 x \mathrm{~V}$, sir $2 \mathrm{~N} 247 \mathrm{~A}-3 \mathrm{x}$ V5, Sir2 $\Delta 1-129-3 x$ V5, or Sir2 $41-129$ N247A-3x V5, selecting for growth on media containing 5-Fluoroorotic Acid (5-FOA), on which cells expressing $\mathrm{ura4}^{+}$cannot grow. Counter-selection was performed on media lacking uracil to confirm the replacement of $\mathrm{ura}^{+}$marker gene. The sir $2-3 x$ V5 locus was checked by sequencing and strains were outcrossed once before further characterization.

The $h t b 14 Q / 4 R$ mutants were made by first creating a diploid fission yeast strain containing both an untagged version of $h t b 1^{+}$and htb1-1x Flag linked to kanamycin resistance (KanR). The htbl of the htb-1x Flag locus of the diploid strain was then replaced with the $\mathrm{ura}^{+}$marker gene. The presence of $\mathrm{ura}^{+}$was monitored by selection on media lacking uracil and counter selected on media containing 5-FOA. Next, the $\mathrm{ura}^{+}$marker gene was replaced with $h t b 1 \mathrm{~K} 5,6,10,15 \mathrm{Q} / R$, and strains were selected based on the loss of the $\mathrm{urat}^{+}$marker. Strains were then converted back to haploid and selected for KanR. The $h t b l$ locus was sequenced and strains were outcrossed two times before use.

The ded1-1D5 cig2-3x HA and ded1-61 cig2-3x HA strains previously described were obtained from Beata Grallert (Oslo University Hospital) [162]. The leul-32 mutation was introduced into the ded1-1D5 cig2-3x HA strain background. The ded1 mutant strains were transformed with the pREP-41 DDX3X-3x V5-LEU2 ${ }^{+}$constructs and maintained on media lacking uracil and grown at the appropriate permissive temperature for Ded1 function before use in experiments.

\section{Plasmid Construction}

All plasmids used in this study are described in Table A-2. The pREP81-Sir2-3x V5 construct was produced by cloning Sir2 amplified from cDNA into JP1611 using XhoI and BglII as previously described [83]. The Sir2 N247A mutation was introduced into the plasmid by PCR [83]. To create JP2302 (pREP81-Sir2-3x V5) and JP2304 (pREP81-Sir2-3x V5), a frameshift mutation was corrected from the original plasmid

previously generated. These plasmids were used to make specific Sir2 truncations and 
mutations by inverse PCR. All plasmid constructs were verified by sequencing before being used in experiments.

The pGEX-KG-GST-Sir2 recombinant protein expression constructs were created by cloning Sir2 into JP81 using EcoRI as previously described [83]. GST-Sir2

truncations were made using inverse PCR or cloning using Clontech Laboratories Inc InFusion ${ }^{\circledR}$ cloning system. The plasmids were sequenced before use in protein expression.

For cloning fission yeast $d e d 1^{+}$and human $D D X 3 X$, a LEU2 marked JP1340 pREP41-3xV5 expression vector was used [194]. To make the pREP41-Ded1-3x V5 plasmid, ded $1^{+}$was amplified from $S$. pombe genomic DNA and cloned into vector using SalI by Clontech Laboratories Inc In-Fusion ${ }^{\circledR}$ cloning. To produce the pREP41-DDX3X$3 \mathrm{x}$ V5 plasmid, $D D X 3 X$ amplified from previously cloned $D D X 3 X \mathrm{cDNA}$ (gift from J. Paul Taylor, St. Jude Children's Research Hospital) and inserted into JP1340 using SalI. Mutations of DDX3X were made using inverse PCR mutagenesis. Sequencing of ded1 and $D D X 3 X$ was performed before the plasmids were used in experiments.

\section{Silencing Assay}

Cells were grown overnight to a density of $4.0 \times 10^{6}$ cells $/ \mathrm{mL}$ at $25^{\circ} \mathrm{C}$ in YES or PMG media with the appropriate supplements. The cells were then washed and resuspended to a density of $3 \times 10^{6}$ cells $/ \mathrm{mL}$ in PMG. Next, four subsequent 1:5 dilutions were made to generate a five-fold serial dilution in PMG lacking supplements. Cells were spotted onto PMG plates supplemented with uracil, PMG plates with no uracil supplement, and plates containing $2 \mathrm{mg} / \mathrm{mL} 5$-FOA using a multichannel pipet. The plates were incubated for three to five days at $25^{\circ} \mathrm{C}$ or $33^{\circ} \mathrm{C}$ before photographing with a Biorad Gel Doc XR imaging system.

\section{Protein Extraction and Western Blotting}

Cells were grown to a density of $5 \times 10^{6}$ cells $/ \mathrm{mL}$ in $25 \mathrm{~mL}$ of YES or PMG media containing the proper supplements. Cells were collected by centrifugation and washed before storage at $-80^{\circ} \mathrm{C}$ or immediately lysed by resuspending the cells to a density of $5 \mathrm{x}$ $10^{5}$ cells $/ \mathrm{mL}$ in $2 \mathrm{X}$ SDS sample buffer (20\% glycerol, $120 \mathrm{mM}$ Tris $\mathrm{HCl} \mathrm{pH} 6.8,4 \%$ SDS, and $2.5 \% \beta$-mercaptoethanol) before bead beating using a Mini Beadbeater ${ }^{\mathrm{TM}}$. Cell debris was cleared by centrifugation at 13,000 RPM for 10 minutes and a 1:5 dilution of the extract was made in $2 \mathrm{X}$ SDS sample buffer.

Western blotting was performed by running $10 \mathrm{ul}$ of the protein extract on an Invitrogen ${ }^{\mathrm{TM}} \mathrm{NuPage}^{\mathrm{TM}}$ gel in $1 \mathrm{X}$ MOPs running buffer at 150 volts for roughly $11 / 2$ hours. Transfer of the proteins to GE Healthcare Amersham ${ }^{\mathrm{TM}}$ Protran $^{\mathrm{TM}} 0.45 \mu \mathrm{m}$ nitrocellulose was performed using $1 \mathrm{X}$ transfer buffer $(47.8 \mathrm{mM}$ Tris base, $38.6 \mathrm{mM}$ glycine, $0.37 \%$ SDS, and 20\% methanol) in an Invitrogen ${ }^{\mathrm{TM}}$ XCell II transfer module for one hour at 35 volts. Blots were then blocked using 5\% milk made in phosphate-buffered 
saline (PBS) containing 0.2\% Tween 20 (PBST) for one hour at room temperature with gentle shaking. Blots were incubated at room temperature for one hour or overnight at $4^{\circ} \mathrm{C}$ with the appropriate antibody at a 1:1000 concentration on a rotating wheel. After incubation, blots were washed in PBST for 30 minutes and secondary antibody was added at a concentration of 1:5000 in 3\% milk in PBST and allowed to incubate for one hour at room temperature on a rotating wheel. Blots were then washed for 30 minutes in PBST and developed by exposing to film or using a LI-COR Odyssey developing system after a one minute incubation with Millipore ECL.

\section{RNA Prep for qRT-PCR}

Cells were grown overnight in 50mL of YES or PMG with the appropriate supplements to a density of $4.0 \times 10^{6}$ cells $/ \mathrm{mL}$ at $25^{\circ} \mathrm{C}$. The cells were collected by centrifugation and lysed by bead beating using a Mini Beadbeater ${ }^{\mathrm{TM}}$. Two extractions were performed using 5:1 phenol chloroform $\mathrm{pH} 4.7$ and one extraction was performed using chloroform, each time transferring the aqueous phase to a new microcentrifuge tube. RNA was precipitated by adding three volumes of 200 proof ethanol before centrifuging at $13,000 \mathrm{RPM}$ and $4{ }^{\circ} \mathrm{C}$ for 20 minutes. The pellet was air dried and resuspended in $1 \mathrm{X}$ TE buffer made with Diethylpyrocarbonate (DEPC) treated $\mathrm{H}_{2} \mathrm{O}$. DNAsing was carried out using an Ambion Turbo ${ }^{\mathrm{TM}}$ DNAse kit on $25 \mathrm{ug}$ of RNA at $37^{\circ} \mathrm{C}$ for one hour. After DNAsing, random priming of 5ug of total RNA was performed and 140 ng of random primed RNA was used to make cDNA using Invitrogen ${ }^{\text {TM }}$ Superscript II reverse transcriptase.

\section{Quantitative Real Time PCR}

Quantitative real time PCR (qRT-PCR) was performed to measure $d h$ transcripts (JPO769 CCAGACCATTACAAGCACTACATACG and JPO770 GAATCTTCTCTTG AATAAAACCGCC), $t$ th transcripts (JPO816 CGTTTTTGATACCGGCGC and JPO819 TTGCCGTAACGACATCATGG), and $a d h$ transcripts as the euchromatic control (JPO793 AACGTCAAGTTCGAGGAAGTCC and JPO794 AGAGCGTGTAAATCGGT GTGG). qRT-PCR was performed using an Eppendorf Mastercycler ep Realplex machine and Qiagen Quantifast Sybr ${ }^{\circledR}$ Green. The linear range for each primer set was verified and only ct values which amplified the desired product with $\geq 90 \%$ efficiency were used for qRT-PCR. Transcripts were normalized to $a d h$ and the $\Delta \Delta$ ct method was used to analyze transcript levels. Changes in transcript level were plotted as fold change over WT. Standard error of the means (SEM) was used to generate error bars.

\section{Establishment Assay}

To examine establishment defects in mutants of interest, establishment assays were performed as previously described [79, 83, 84]. Strains containing the mutants were crossed with a $\operatorname{clr} 4 \Delta$ strain to create a double mutant strain. Once the deletion of $\operatorname{clr} 4^{+}$ 
was confirmed, $\mathrm{clr} 4^{+}$was reintroduced into the endogenous locus by digesting and transforming a plasmid containing $\operatorname{clr} 4^{+}$and its targeting sequence along with a traceable ade $6^{+}$marker to monitor integration. Sole copy integration of $\mathrm{clr} 4^{+}$was confirmed by southern analysis using a radioactively labeled probe for $\mathrm{clr} 4^{+}$. Stains were then analyzed by silencing assay or qRT-PCR.

\section{Purification of Recombinant Protein}

A $2 \mathrm{~mL}$ culture of Invitrogen ${ }^{\mathrm{TM}}$ BL-21 E. coli cells transformed with pGEX-KG plasmid containing protein of interest was grown overnight at $37^{\circ} \mathrm{C}$ in Luria Broth with ampicillin (LB-AMP). From this starter culture, $500 \mu 1$ was used to prepare a $200 \mathrm{~mL}$ culture in LB-AMP which was grown for three hours at $34^{\circ} \mathrm{C}$ to an OD of 0.3 . At this point a sample was taken as an uninduced control and the remaining culture was induced using $0.1 \mathrm{mM}$ Isopropyl $\beta$-D-1-thiogalactopyranoside and grown for an additional four to six hours at $25^{\circ} \mathrm{C}$. After incubation, cells were collected by centrifugation and washed with PBS before storing at $-80^{\circ} \mathrm{C}$. Small samples of uninduced and induced culture were run on an Invitrogen ${ }^{\mathrm{TM}} \mathrm{NuPage}^{\mathrm{TM}}$ gel and coomassie stained to monitor induction efficiency.

Cells were lysed by resuspending in five volumes of PBS containing Roche protease inhibitor and sonicated on ice for a total of four minutes. Next 0.1 volumes of 2.5 $\mathrm{M} \mathrm{KCl}$ and DTT to $15 \mathrm{mM}$ were added and the samples were centrifuged at 13,000 $\mathrm{RPM}$ for 10 minutes at $4^{\circ} \mathrm{C}$. One $\mathrm{mL}$ of Pierce glutathione agarose beads washed in PBSKD (PBS, 250M KCl, and $15 \mathrm{mM}$ DTT) were added per $100 \mathrm{~mL}$ lysate equivalent. This was incubated at $4^{\circ} \mathrm{C}$ for 30 minutes with rotation before collection of the beads by centrifugation and washing five times in $1 \mathrm{~mL}$ of PBSKD. The supernatant was removed and the beads were resuspended in $250 \mu \mathrm{l}$ of glutathione buffer $(5 \mathrm{mM}$ glutathione, $50 \mathrm{mM}$ Tris $\mathrm{HCl} \mathrm{pH} \mathrm{8.0,} \mathrm{and} 0.25 \mathrm{M} \mathrm{KCL}$ in $\mathrm{H}_{2} \mathrm{O}$ ) and incubated for five minutes with rotation at $4^{\circ} \mathrm{C}$. The elution was repeated two times and the eluate was pooled and dialyzed against the storage buffer $(100 \mathrm{mM} \mathrm{NaCl}, 50 \mathrm{mM}$ Tris $\mathrm{pH} 8.0$, and $1 \mathrm{mM}$ DTT). Before storage at $-80^{\circ} \mathrm{C}, 10 \%$ glycerol was added to the purified recombinant protein.

\section{Fluor De Lys Assay}

Deacetylase activity of recombinant Sir2 was measured using a Fluor de Lys Green ${ }^{\circledR}$ HDAC drug discovery kit from Enzo Life Sciences as previously described [83]. A $25 \mu 1$ reaction was assembled consisting of $4 \mu \mathrm{M}$ GST-Sir2, GST-Sir2 $\Delta 1-129$, or GSTSir2 $\Delta 1-137,0.5 \mu \mathrm{M}$ Fluor de Lys Green substrate, $8 \mathrm{mM} \mathrm{NAD}^{+}$, and $10.75 \mu 1$ of buffer R $(100 \mathrm{mM} \mathrm{NaCl}, 50 \mathrm{mM}$ Tris $\mathrm{pH} 8.0$, and $1 \mathrm{mM}$ DTT) into a 96 half well black plate. A control reaction was performed using HeLa cell extract. The reaction was covered and incubated at $37^{\circ} \mathrm{C}$ for three hours in the dark. The reaction was then terminated by the addition of $25 \mu \mathrm{l}$ of the quenching and developing reagent which contained $1 \mathrm{mM}$ Nicotinamide and $40 \mu \mathrm{M}$ TSA along with $1.25 \mu \mathrm{l}$ of the $20 \mathrm{X}$ trypsin based developer reagent to the reaction mix. Endpoint fluorescence was measured 15 minutes after adding 
the developer using a Synergy HT BioTek Scanner set at 485nm excitation and 520nm emission. A standard curve was subsequently constructed by serial dilution of the deacetylated fluorogenic substrate, and was used to equate experimentally observed fluorescence units with molar quantities of deacetylated substrate. HDAC activity was plotted as a percentage of WT Sir2 deacetylase activity.

\section{Histone Preparation and UPLC-MS/MS to Detect Acetylation}

Histones were purified following a previously described purification protocol with some modifications [195]. A $150 \mathrm{~mL}$ culture was inoculated to a density of $1.4 \mathrm{X} 10^{6}$ cells/mL in $4 \mathrm{X}$ YES media from a starter culture grown overnight in $4 \mathrm{X}$ YES and grown at $25^{\circ} \mathrm{C}$ overnight. The cells were grown to a density of $3.6 \times 10^{7}$ cells $/ \mathrm{mL}$ and harvested by centrifugation at 3,000 RPM for five minutes in a benchtop centrifuge at $4^{\circ} \mathrm{C}$. Cells were washed with cold $\mathrm{H}_{2} \mathrm{O}$ containing $10 \mathrm{mM}$ sodium butyrate before washing with NIB buffer $(250 \mathrm{mM}$ sucrose, 20mM HEPES pH 7.5, 60mM KCl, 15mM NaCl, $5 \mathrm{mM} \mathrm{MgCl} 2$, $1 \mathrm{mM} \mathrm{CaCl2,0.8 \%}$ Triton X $100,0.5 \mathrm{mM}$ spermine, $2.5 \mathrm{mM}$ spermidine, $10 \mathrm{mM}$ sodium butyrate, $1 \mathrm{mM}$ PMSF, and Sigma yeast protease inhibitor). The cell pellet was frozen on dry ice and stored at $-80^{\circ} \mathrm{C}$. For lysis, the pellet was resuspended in $2 \mathrm{~mL}$ of ice cold NIB and transferred to a bead beater tube along with chilled acid washed glass beads. The sample and beads were frozen on dry ice and bead beat for a total of 10 minutes at max power using a Mini Beadbeater ${ }^{\mathrm{TM}}$. The sample was collected by "piggy backing centrifugation" into a 50mL Oak Ridge tube at 3,000 RPM and $4^{\circ} \mathrm{C}$ in a benchtop centrifuge. The samples were then pelleted by centrifuging at 20,000 RPM for 10 minutes at $4^{\circ} \mathrm{C}$ in a Beckman Avanti centrifuge J-30I centrifuge using a JA25.50 rotor. The supernatant was discarded and the pellet was washed in $15 \mathrm{~mL}$ of NIB. The pellet was then resuspended in $10 \mathrm{~mL}$ of $0.4 \mathrm{~N} \mathrm{H}_{2} \mathrm{SO}_{4}$ and sonicated for one minute at max power before incubation for two hours with rotation at $4^{\circ} \mathrm{C}$. The acid extracted sample was then pelleted by centrifuging at 20,000 RPM at $4^{\circ} \mathrm{C}$ for 10 minutes and the supernatant was transferred to a new tube along with $5 \mathrm{~mL}$ of $5 \%$ buffer $\mathrm{G}(5 \%$ guanidine $\mathrm{HCl}$ and $100 \mathrm{mM}$ potassium phosphate buffer $\mathrm{pH} 6.8$ ) where the $\mathrm{pH}$ was adjusted to 6.8 using $5 \mathrm{~N}$ $\mathrm{KOH}$. $0.5 \mathrm{~mL}$ of Bio-Rex ${ }^{\mathrm{TM}}$ pre-equilibrated in $5 \%$ buffer $\mathrm{G}$ was added to the sample and incubated at room temperature with rotation overnight. The resin was then washed two times with $20 \mathrm{~mL}$ of $5 \%$ buffer $\mathrm{G}$ and incubated with $3 \mathrm{~mL}$ of $40 \%$ buffer $\mathrm{G}(40 \%$ guanidine $\mathrm{HCl}$ and $100 \mathrm{mM}$ potassium phosphate buffer $\mathrm{pH} 6.8$ ) for one hour at room temperature to elute the bound protein. Buffer exchange and concentration was performed against $5 \%$ acetonitrile with $0.1 \%$ TFA to a final volume of $150 \mu 1$ and the sample was stored at $-80^{\circ} \mathrm{C}$. Protein concentration was measured and $10 \mu \mathrm{g}$ of sample was analyzed on a gel for quality control.

MS/MS was performed as previously described by Ryan Henry and Andy Andrews at Fox Chase Cancer Center (FCCC) [196]. Before MS/MS, histones were TCA precipitated and propionylated to chemically modify unmodified or monomethylated lysine residues using propionic anhydride. The histones were then digested with trypsin overnight. After tryptic digestion, MS/MS was performed using a Waters Acquity $\mathrm{H}-$ Class ultra-high performance liquid chromatography (UPLC) coupled to a Thermo TSQ 
Quantum Access triple quadrupole mass spectrometer to quantify modified histone peptides. Selective reaction monitoring was used to monitor the elution of the acetylated and propionylated tryptic digested peptides. Transitions were created to study acetylation of pombe WT and sir2 $\Delta \mathrm{H} 3$ and H4 histone tails (Table A-3) [unpublished communication from Ryan Henry and Andy Andrews on 12/07/2016]. In the data analysis, each acetylated or propionylated peak was identified by retention time and specific transitions. The peak integration was conducted using Xcalibur software and the fraction of a specific peptide was calculated. Error bars were generated using SEM.

\section{Immunoprecipitation of Sir2 and MS/MS for Interactors and PTM}

300mL cultures of cells episomally expressing Sir2-3X V5 or Sir2 $\Delta 1-129-3 X$ V5 constructs were grown to a density of $5 \times 10^{6}-1 \times 10^{7}$ cells $/ \mathrm{mL}$ in PMG lacking leucine. Cells were collected by centrifugation, washed, and stored at $-80^{\circ} \mathrm{C}$. To lyse the cells, 0.4 cell pellet volumes of lysis buffer (50mM HEPES pH 7.5, 300mM NaCl, 20\% glycerol, $0.1 \%$ NP-40, 4mM EDTA, 2mM EGTA, Roche EDTA free protease inhibitor, and $1 \mathrm{mM}$ PMSF) was used to resuspend the cell pellet. The cells were then ground with a prechilled mortar and pestle using liquid $\mathrm{N}_{2}$ until $70 \%$ lysis was achieved. The frozen yeast powder was thawed on ice and lysis buffer containing 60 units of Roche DNAse I and $2 \mathrm{mM} \mathrm{MgCl} 2$ was added to make a final volume of $1 \mathrm{~mL}$. This mixture was incubated on ice for 10 minutes before centrifuging at $21,000 \mathrm{x}$ g at $4{ }^{\circ} \mathrm{C}$ for 30 minutes. Protein concentrations were measured using Bio-Rad Bradford solution and the amount of protein was normalized between samples. An input aliquot of $1 \%$ of the material was retained as input. Equal amounts of protein extract were then added to pre-equilibrated Sigma anti-V5 affinity agarose and incubated for two hours at $4^{\circ} \mathrm{C}$ with rotation. The beads were then gently centrifuged and the supernatant was removed. The beads were washed four times with $1 \mathrm{~mL}$ of lysis buffer. The protein was eluted by incubating with lysis buffer containing $0.4 \mathrm{mg} / \mathrm{mL} 3 \mathrm{X}$ V5 peptide incubating for 30 minutes. This was repeated two times. The samples were analyzed by both coomassie staining and western blotting, incubating overnight with an anti-V5 monoclonal antibody (AbD Serotec MCA1360) at a concentration of 1:1000 in PBST.

MS/MS analysis was performed by Alan Tackett and Stephanie Byrum at the University of Arkansas Medical School (UAMS) on the Sir2 IP samples to examine both interactors and PTM. Proteins were resolved by SDS-PAGE on a 4-20\% glycine gel and visualized by coomassie staining. Gel bands were then sliced into $2 \mathrm{~mm}$ sections and destained in 50\% methanol with $100 \mathrm{mM}$ ammonium bicarbonate followed by reduction in $10 \mathrm{mM}$ Tris[2-carboxyethy]phosphine and alkylation in $50 \mathrm{mM}$ iodoacetamide. The gel slices were then dehydrated in acetonitrile before chymotrypsin digestion overnight at $37^{\circ} \mathrm{C}$. Peptide products were then acidified in $0.1 \%$ formic acid before MS/MS analysis.

Tryptic peptides were separated using a Waters UPLC system. Eluted peptides were ionized by electrospray followed by MS/MS analysis using high-energy collision induced dissociation (HCD) or collision induced dissociation (CID) on an Orbitrap Fusion Tribrid mass spectrometer. Proteins were identified by database searching using 
Mascot. Scaffold was used to verify MS/MS based protein and peptide identification, using a false discovery rate of less than $1.0 \%$. Peptides obtained from the MS/MS analysis to examine potential Sir2 interactors are listed in Table A-4 [unpublished communication from Alan Tackett 11/23/2015]. The results are sorted based on the number of peptides identified in the sir2-3x V5 sample. For analysis of PTMs found on Sir2, two different MS/MS were used: Collision Induced Dissociation (CID) and HighEnergy Collisional Dissociation (HCD). Peptides found to be phosphorylated in Sir2 are listed in Table A-5 [unpublished communication from Alan Tackett on 10/01/2015]. The phosphorylated peptides from each analysis method are listed and phosphorylated residues are in red.

\section{Chromatin Immunoprecipitation}

Chromatin immunoprecipitation (ChIP) was performed as previously described [83]. To determine the appropriate concentration of antibody for each ChIP, ChIP was performed using a titration of each antibody to determine the concentration giving optimal enrichment. Cells were grown overnight to a density of $6 \times 10^{6}$ cells $/ \mathrm{mL}$ in PMG lacking leucine. $45 \mathrm{~mL}$ of cells were fixed at a final concentration of $3 \%$ PFA (paraformaldehyde), incubating at room temperature for 18 minutes with gentle shaking. The fixation was stopped by adding $5 \mathrm{~mL}$ of $2.5 \mathrm{M}$ glycine and incubating five minutes at room temperature. Cells were pelleted by centrifugation at $3000 \mathrm{RPM}$ at $4{ }^{\circ} \mathrm{C}$ and washed two times before being lysed or stored at $-80^{\circ} \mathrm{C}$.

Cell lysis was performed by the addition of 400ul ChIP lysis buffer [50mM HEPES-NaOH pH 7.5, 150mM NaCl, $1 \mathrm{mM}$ EDTA, 1\% Triton X-100, 0.1\% deoxycholate (DOC)] and acid washed glass beads by bead beating. The chromatin was then sheared by probe sonication using three rounds of 20 second sonication at maximum power on ice at $4^{\circ} \mathrm{C}$. Cell debris was then cleared by centrifugation at 13,000 RPM for 15 minutes at $4^{\circ} \mathrm{C}$. A $20 \mathrm{ul}$ sample was taken as the input control and stored at $-20^{\circ} \mathrm{C}$.

Half of each lysate was used for ChIP along with the appropriate amount of antibody. The samples were then incubated overnight at $4{ }^{\circ} \mathrm{C}$ with rotation. Next, 50ul of protein $\mathrm{A}$ or $\mathrm{G}$ Dynabeads ${ }^{\mathrm{TM}}$ (Invitrogen ${ }^{\mathrm{TM}}$ ) were added to each lysate and incubated for one hour at $4{ }^{\circ} \mathrm{C}$ with rotation. The beads were then washed two times with $1 \mathrm{~mL}$ of ChIP Lysis Buffer, $1 \mathrm{X}$ with $1 \mathrm{~mL}$ of 0.5M ChIP Lysis Buffer [50mM HEPES-NaOH pH 7.5, $0.5 \mathrm{M} \mathrm{NaCl}, 1 \mathrm{mM}$ EDTA, $1 \%$ Triton X-100, 0.1\% DOC], $1 \mathrm{X}$ with $1 \mathrm{~mL}$ of ChIP Wash Buffer [10mM Tris-HCl pH 8.0, 250mM LiCl, 1mM EDTA, 0.5\% Igepal CA-630, 0.5\% DOC], and one time with $1 \mathrm{~mL}$ of $1 \mathrm{X} \mathrm{TE}$. Each wash was incubated for five minutes at room temperature with rotation. After each wash, the beads were recovered by centrifugation at $2,500 \mathrm{RPM}$ at $4^{\circ} \mathrm{C}$ for 30 seconds and placed on a magnetic rack to aspirate off all supernatant.

The ChIP and input samples were then decrosslinked by resuspending in $250 \mu 1$ $1 \mathrm{X}$ TES [1X TE with $0.5 \% \mathrm{SDS}$ ] and incubated overnight at $65^{\circ} \mathrm{C}$. The sample volume was brought to $500 \mathrm{ul}$ by the addition of $1 \mathrm{X}$ TE and the serine protease Proteinase $\mathrm{K}$ was 
added to a concentration of $0.8 \mathrm{mg} / \mathrm{mL}$ and then incubated at $37^{\circ} \mathrm{C}$ for one hour. This step cleaves peptide bonds allowing for the degradation of contaminating proteins. The supernatant was cleaned up using the Wizard ${ }^{\circledR}$ DNA Clean-Up System (Promega) and samples were then analyzed using qRT-PCR.

\section{Sir2 Immunofluorescence}

Immunofluorescent analysis of localization was carried out as previously described [197]. A 25mL culture of fission yeast was grown in PMG lacking leucine to a density of $5 \times 10^{6}$ cells $/ \mathrm{mL}$. Cells were fixed at a final concentration of $3.8 \%$ PFA at $18^{\circ} \mathrm{C}$ for 30 minutes. Cells were then centrifuged at 3,000 RPM for five minutes and washed in $15 \mathrm{~mL}$ of PEM (100mM Pipes pH 6.9, $1 \mathrm{mM}$ EDTA, $1 \mathrm{mM} \mathrm{MgSO}_{4}$, and $5 \mathrm{M}$ $\mathrm{NaOH}$ to adjust the $\mathrm{pH}$ to 6.9) two times. The cells were then transferred to a microcentrifuge tube and washed two times in $1 \mathrm{~mL}$ of PEM before resuspending to a density of $1 \times 10^{8}$ cells $/ \mathrm{mL}$ in $1 \mathrm{mg} / \mathrm{mL}$ zymolyase in PEMS (PEM with $1.2 \mathrm{M}$ sorbitol) and incubating at $36^{\circ} \mathrm{C}$ for 90 minutes until the cell wall was perforated. The cells were then gently spun down and incubated for five minutes at room temperature in $200 \mu 1$ of PEMS with $1 \%$ Triton-X 100 . Next, the cells were spun down and washed in $1 \mathrm{~mL}$ of PEM a total of four times. The cells were then incubated for 30 minutes in $0.5 \mathrm{~mL}$ PEMBAL (PEM with $1 \%$ bovine serum albumin (BSA), $0.1 \%$ sodium azide, and $100 \mathrm{mM}$ lysine $\mathrm{HCl}$ ) on a rotating wheel. Next, $100 \mu \mathrm{l}$ of the cells were incubated overnight with rotation at $4^{\circ} \mathrm{C}$ with Serotec anti-V5 antibody at a 1:100 concentration.

The next day, cells were collected by centrifugation and the antibody solution was removed before washing cells three times with a 30 minute incubation on a rotating wheel with $1 \mathrm{~mL}$ of PEMBAL for each wash. The cells were centrifuged at 8,000 RPM for one minute and all supernatant was removed. Cells were then resuspended in $100 \mu 1$ PEMBAL and the conjugated Alexa Fluor ${ }^{\circledR} 594$ secondary donkey anti-mouse antibody was added at a 1:100 dilution. This was incubated for 4 hours at room temperature in the dark on a rotating wheel. The cells were then washed in $1 \mathrm{~mL}$ of PEMBAL for 30 minutes at room temperature on a rotating wheel before washing two times with $1 \mathrm{~mL}$ of PBS azide (PBS containing $0.1 \%$ sodium azide) for 15 minutes with rotation. Cells were then resuspended in $30 \mu 1$ of PBS azide and $8 \mu \mathrm{g} / \mathrm{mL}$ 4',6'-Diamidino-2-phenylindole (DAPI). Around $7 \mu \mathrm{l}$ of this mixture was added to $150 \mu \mathrm{l}$ of PBS azide and cytospun onto polylysine-coated slides. A coverslip was mounted using Vectashield mountant with nail polish to seal around the edges. Slides were visualized using a Zeiss Axioskop II microscope with the appropriate filters. A Coolsnap HQ camera was used to capture the images.

\section{Electromobility Shift Assay}

To examine nucleic acid binding of Sir2, 15 $\mu \mathrm{g}$ of recombinant GST, GST-Sir2 1128 , or GST-Chp1 CD were incubated with $200 \mathrm{nmol}$ of dsDNA or ssRNA containing a 3 '-fluorescein tag in a $20 \mu 1$ reaction containing $20 \mathrm{mM}$ HEPES pH 7.5, 100mM KCl, 
$0.02 \%$ NP-40, $0.5 \mathrm{mM}$ DTT, and 5U of Promega RNasin. The reactions were carefully mixed and incubated on ice for 30 minutes [198]. After incubation, $2 \mu 1$ of $100 \%$ glycerol was added to each sample and mixed gently by pipetting. The entire reaction was then loaded onto Invitrogen ${ }^{\mathrm{TM}}$ Novex $^{\circledR} 4-20 \%$ TBE gels and run for $1 \frac{1}{2}$ hours in cold $0.5 \mathrm{X}$ TBE running buffer. The gels were imaged using a Fujifilm Las-4000 Luminescent Imager with the $\mathrm{SyBr}$ green setting with a two-minute exposure. After imaging, coomassie staining was performed to ensure equal loading for each protein.

\section{RNA Purification and RNA-Seq}

Hot phenol extraction was used to prepare the RNA [199]. Cultures were grown overnight in YES at $25^{\circ} \mathrm{C}$ to a density of $2.5 \times 10^{6}$ cells $/ \mathrm{mL}$ in a $25 \mathrm{~mL}$ culture. The cells were pelleted by centrifugation and washed in DEPC $\mathrm{H}_{2} \mathrm{O}$. The pellet was resuspended in 750ul TES Buffer [50mM Tris-HCl pH 7.5, 10mM EDTA, 100mM NaCl, 0.5\% SDS made in DEPC $\mathrm{H}_{2} \mathrm{O}$ ] along with an equal volume of 5:1 phenol:chloroform $\mathrm{pH} 4.7$ and incubated at $65^{\circ} \mathrm{C}$ for one hour with vortexing for 10 seconds every 10 minutes. The samples were then cooled on ice and centrifuged for five minutes at 13,000 RPM. The aqueous phase was transferred to a $2 \mathrm{~mL}$ phase lock tube and an additional phenol:chloroform extraction was performed. After centrifugation, the aqueous phase was transferred to a new tube and an equal volume of chloroform was added. To precipitate the RNA, the aqueous phase was transferred to a $2 \mathrm{~mL}$ microcentrifuge tube and three volumes of ice cold ethanol and $3 \mathrm{M} \mathrm{NaAc} \mathrm{pH} 5.2$ were added and the samples were kept at $-20^{\circ} \mathrm{C}$ to precipitate the RNA. The next day, samples were centrifuged at $14,000 \mathrm{RPM}$ and $4^{\circ} \mathrm{C}$ for 15 minutes to pellet the RNA. The pellet was washed with ice cold $70 \%$ ethanol and air dried for 30 minutes. A Turbo ${ }^{\mathrm{TM}}$ DNAse (Ambion) reaction was set up with 100ug of RNA in a 150ul reaction containing 5ul of Turbo ${ }^{\mathrm{TM}}$ DNAse. The reaction was incubated at $37^{\circ} \mathrm{C}$ for 30 minutes and another $5 \mathrm{ul}$ of Turbo ${ }^{\mathrm{TM}}$ DNAse was added and incubated for an additional 30 minutes prior to the removal of the DNAse using 50ul of inactivation beads. An RNeasy Mini kit (Sigma) was used to further purify and concentrate the RNA which was eluted in a final volume of 30 ul of DEPC $\mathrm{H}_{2} \mathrm{O}$. Quantitative real time PCR was performed as previously described on cDNA prepared from the random priming of 5 ug of DNAsed RNA [149].

The samples were prepared in triplicate and RNA-seq was performed by the St. Jude Hartwell Center. RNA-seq was done using ribosomal RNA depleted samples. RNA was quantified using a Quant-iT ${ }^{\mathrm{TM}}$ assay (Life Technology). The quality was checked by 2100 Bioanalyzer RNA 6000 Nano assay (Agilent) or LabChip RNA Pico Sensitivity assay (PerkElmer) before library generation. Libraries were prepared from $2 \mathrm{ug}$ of RNA. Ribosomal RNA was removed from the samples using Ribo-Zero ${ }^{\mathrm{TM}}$ Gold rRNA Removal Kit (Yeast) following manufacturer instructions (Illumina). Libraries were prepared from the ribosomal RNA depleted samples using the TruSeq Strand Total RNA Library Prep Kit, beginning at Elution 2 - Fragment - Prime step immediately preceding cDNA synthesis according to the manufacturer instructions (Illumina) with the following modifications; the $94 \mathrm{C}$ Elution 2 - Fragment - Prime incubation was reduced to five minutes and the PCR was reduced to 11 cycles. Libraries were quantified using the 
Quant-iT ${ }^{\mathrm{TM}}$ PicoGreen dsDNA assay (Life Technologies) or Kapa Library Quantification kit (Kapa Biosystems). One hundred cycle paired end sequencing was performed on an Illumina HiSeq 2500. Three biological replicates were used for each strain analyzed [149].

The total RNA was sequenced using stranded protocol with $2 \times 100 \mathrm{bp}$ setting. Bioinformatic analysis was performed by Chunxu Qu (Department of Bioinformatics, St. Jude Children's Research Hospital). The paired end reads were first trimmed $10 \mathrm{bp}$ at the 5'-end and then mapped to $S$. pombe (ftp://ftp.ebi.ac.uk/pub/databases/pombase/pombe/C hromosome_Dumps/Schizosaccharomyces_pombe. ASM294v2.20.dna.genome.fa) genome using GSNAP (version 2013-02-25), with uniquely and multiply mapped reads in separate files, since fission yeast has large centromeres. Fragments uniquely mapped to each gene were counted using an in-house perl script and FPKM (fragments per kilobase of exon per million fragments mapped) were calculated. Fragments that mapped to each telomere or centromere were also counted with both uniquely and multiply mapped reads, and FPM (fragments per million total fragments) were calculated. FPKM values were quantile normalized and differentially expressed genes were analyzed using limma/Bioconductor. The regions used for centromere analysis were chr1:37536873789421, chr2:1602264-1644747, chr3:1070904-1137003 [149].

\section{Histone Purification and MS/MS to Examine PTMs in clr3 $\Delta$ Cells}

Histones were purified as previously described with slight modification [195]. Cultures were grown overnight in 2L of 4X YES from a starter culture to a density of 3.6$4 \times 10^{7}$ cells $/ \mathrm{mL}$. Cells were collected by centrifugation at 3,000 RPM at $4^{\circ} \mathrm{C}$ and washed once in $\mathrm{H}_{2} \mathrm{O}$ containing $10 \mathrm{mM}$ sodium butyrate and once in NIB buffer before storing at $80^{\circ} \mathrm{C}$. To lyse the cells, the pellet was resuspended in $15 \mathrm{~mL}$ of NIB buffer and bead beaten with acid washed glass beads for a total of 6 minutes using a Mini Beadbeater ${ }^{\mathrm{TM}}$. The lysate was cleared by centrifuging at 20,000 RPM for 20 minutes at $4{ }^{\circ} \mathrm{C}$ using a Beckman Avanti centrifuge J-30I centrifuge using a JA25.50 rotor and the pellet was washed two times in NIB buffer. The pellet was then resuspended in $20 \mathrm{~mL}$ of $0.4 \mathrm{~N}$ $\mathrm{H}_{2} \mathrm{SO}_{4}$ and sonicated for 90 seconds before incubating for two hours at $4{ }^{\circ} \mathrm{C}$. The acid insoluble material was cleared by centrifugation and the acid soluble material was collected. The $\mathrm{pH}$ was adjusted to 6.8 by adding $20 \mathrm{~mL}$ of $5 \%$ buffer $\mathrm{G}$ and $5 \mathrm{~N} \mathrm{KOH}$ and then incubated with $1.5 \mathrm{~mL}$ of pre-equilibrated Bio-Rex ${ }^{\mathrm{TM}}$ overnight at room temperature. The resin was then washed twice with $20 \mathrm{~mL}$ of buffer $\mathrm{G}$ with 15 -minute incubation and rotation for each wash. Material bound to the resin was eluted by adding $3 \mathrm{~mL}$ of $40 \%$ buffer $\mathrm{G}$ and rotating for one hour at room temperature. The eluate was collected and buffer exchange and concentration was performed against $5 \%$ acetonitrile with $0.1 \%$ TFA using an Amicon Pro purification system with a $3 \mathrm{kDa}$ cutoff to a final volume of $500 \mu 1$. The concentration was measured using Bio-Rad Bradford assay solution.

The samples were then run on a $\mathrm{C} 4$ reverse phase HPLC (Grace Vydac $^{\circledR} 214 \mathrm{TP} 104250 \times 4.6,10 \mu \mathrm{m}, 300 \AA$ ) column which was pre-equilibrated for 30 minutes with $5 \%$ acetonitrile containing $0.1 \%$ TFA. The samples were loaded onto the 
column mounted onto a Water HPLC system and eluted by a linear acetonitrile gradient of $30-65 \%$ buffer B (acetonitrile with $0.09 \%$ TFA) and delivered at a flow rate of $1 \mathrm{~mL} /$ minute for 50 minutes and samples were collected in $1 \mathrm{~mL}$ fractions [195]. Two runs were performed in order to inject $6.4 \mathrm{mg}$ of protein for a total of $2 \mathrm{~mL}$ fractions. Once collected, $10 \mu 1$ of $\beta$-mercaptoethanol was added to each $1 \mathrm{~mL}$ fraction and the fraction was frozen on dry ice before being lyophilized. The lyophilized fractions were resuspended in $100 \mu \mathrm{l}$ of ultra-pure $\mathrm{H}_{2} \mathrm{O}$ and the histone containing fractions were identified by dot blot on GE Healthcare Amersham ${ }^{\mathrm{TM}} 0.45 \mu \mathrm{m}$ Protran membrane using anti H2A (Active Motif 39235), Anti H2B (Bio Academia 63-125), Anti H3 (Active Motif 39163), and Anti H4 (Active Motif 39270). The blots were developed using LICOR.

The fractions which contained histones were pooled together and run on a C18

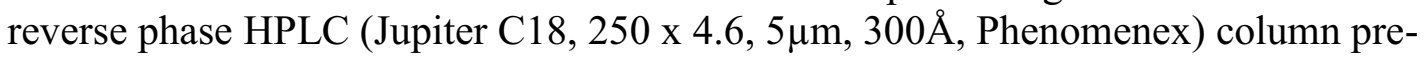
equilibrated for 30 minutes with $5 \%$ acetonitrile containing $0.1 \%$ TFA. The samples were injected and separated using a multistep acetonitrile gradient at a flow rate of $1 \mathrm{~mL} /$ minute using buffer B with an acetonitrile gradient of $20-38 \%$ for the first 25 minutes, then $38-48 \%$ for 25 minutes, and $60 \%$ for the last 10 minutes. The sample was run once for a total of $1 \mathrm{~mL}$ fractions which were lyophilized and resuspended in $50 \mu 1$ of ultra-pure $\mathrm{H}_{2} \mathrm{O}$ [195]. Dot blotting was performed as above and samples were run on a $12 \%$ Invitrogen $^{\mathrm{TM}} \mathrm{NuPage}^{\mathrm{TM}}$ gel and coomassie stained. The relevant fractions were frozen and sent for mass spectrometry to examine PTMs.

The histone samples from WT and $\operatorname{clr} 3 \Delta$ cells were treated with propionic anhydride which chemically modifies unmethylated and monomethylated lysines. The propionylated histones were then digested with trypsin. The trypsin cleaved peptides were then subjected to differential in vitro chemical derivatization using $d_{0}$ propionic anhydride for the WT strain and $d_{10}$ propionic anhydride for the $\operatorname{clr} 3 \Delta$ strain in order to differentially label the peptides from the two strains. The $d_{10}$ derivatization produces a 5Da mass shift to the clr3 $\Delta$ sample to allow for differentiation between samples. The protein concentration was determined for each sample and mixed in a 1:1 ratio before MS/MS. High resolution LC-MS/MS was done on a Thermo Velos ETD Orbitrap mass spectrometer. Modified peptides were quantified based on a mass shift for precursor ion mass between WT and clr3 $\Delta$. The changes in acetylation were plotted as acetylation in the $c \operatorname{lr} 3 \Delta$ strain relative to WT to indicate the fold change in each PTM [200].

\section{Measuring Ded1 Protein Levels}

Temperature shift assays on ded1-1D5 cultures were performed by growing $100 \mathrm{~mL}$ of cells in PMG lacking leucine to a density of $4.0 \times 10^{6}$ cells $/ \mathrm{mL}$ at $25^{\circ} \mathrm{C}$. The culture was split into $25 \mathrm{~mL}$ cultures, and one culture was kept at $25^{\circ} \mathrm{C}$ while the other was shifted to $36^{\circ} \mathrm{C}$ for three hours. For ded1-61 cultures, cells were grown to a density of $\sim 4.0 \times 10^{6}$ cells $/ \mathrm{mL}$ at $32^{\circ} \mathrm{C}$ and were split and cultured at $20^{\circ} \mathrm{C}$ or $36^{\circ} \mathrm{C}$ for three hours. Cells were collected by centrifugation at 3,000 RPM at $4^{\circ} \mathrm{C}$ and lysed for protein extraction as described above [157]. 
Total cell extracts and western blotting was performed as described above using the following mouse monoclonal antibodies: anti-V5 antibody (AbD Serotec MCA1360) and anti-tubulin antibody (kind gift from K. Gull lab) at dilutions of 1:2000 in PBST for one hour at room temperature. Rat monoclonal anti-HA 3F10 antibody (Roche) was used at a dilution of 1:500 overnight at $4^{\circ} \mathrm{C}$. Rabbit polyclonal anti-Ded1p antibody (kind gift from Nancy Walworth) was used at a dilution of 1:50,000 overnight at $4{ }^{\circ} \mathrm{C}$. Secondary incubation and developing was performed as described above [157, 201].

\section{Measuring Ded1 Transcript Levels}

For ded1-1D5, 50mL of cells were grown to a density of $4.0 \times 10^{6}$ cells $/ \mathrm{mL}$ at $25^{\circ} \mathrm{C}$ overnight in PMG lacking leucine. The next day, the cultures were split and grown at $25^{\circ} \mathrm{C}$ or $36^{\circ} \mathrm{C}$ for 2.5 hours. For ded1-61 cultures, cells were grown at $32^{\circ} \mathrm{C}$ prior to splitting and growing at $20^{\circ} \mathrm{C}$ or $36^{\circ} \mathrm{C}$ for three hours. Cells were collected by centrifugation at 3,000 RPM at $4 \mathrm{C}$ before washing in $1 \mathrm{X}$ TE. Random priming of total cellular RNA was used to prepare cDNA as and quantitative real-time PCR was performed to measure transcript levels of $\mathrm{cig}^{+}{ }^{+}$(JPO 2990 TTTGTTTAATGCCCGAAA CC and JPO 2991 TGCTAGCGATGAGAAGAGCA) and $a d h 1^{+}$as the euchromatic control as described above [157]. 


\section{CHAPTER 3. IMPORTANCE OF THE SIR2 NTD IN HETEROCHROMATIN ESTABLISHMENT}

\section{Role of the Sir2 NTD in Heterochromatin Maintenance}

It has previously been shown that deletion of Sir2 causes only a minimal impact on the maintenance of pericentromeric heterochromatin but is necessary for its establishment. In contrast, the Sir2 N247A catalytic HDAC mutant shows some ability to establish heterochromatin de novo [83]. This led us to question whether other domains of Sir2 outside of the HDAC domain are involved in heterochromatin formation.

To address this question, we performed a truncational analysis of Sir2 to elucidate whether its large auxiliary NTD and CTD play a role in heterochromatin formation. The HDAC domain of Sir2 stretches from residue 147 to residue 432 (Figure 3-1A). In order to examine the role of domains outside the HDAC core, we constructed a series of NTD and CTD deletions of Sir2 in a pREP41 episomal expression vector with a 3x V5 Cterminal tag and transformed them into a sir $2 \Delta$ strain to monitor their ability to complement for Sir2 function.

Unfortunately, the CTD truncations of Sir2 exhibited high levels of protein instability leading us to continue our study with only the NTD truncations. A large truncation, Sir2 $\Delta 1-129$, was constructed in both the WT Sir2 and Sir2 N247A vectors. This deletion is not predicted to impact the HDAC activity of Sir2 since it lies outside of the catalytic HDAC core. Both the Sir2 $\Delta 1-129$ and Sir2 $\Delta 1-129$ N247A construct were stably expressed as shown by immunoblotting (Figure 3-1B). While Sir2 has not been implicated in the maintenance of centromeric silencing, we found that at the elevated temperature of $33^{\circ} \mathrm{C}$, our $\operatorname{sir} 2 \Delta$ strain is slightly defective for the transcriptional repression of a centromeric reporter gene, otrlR::ura $4^{+}$(Figure 3-1C). This is likely due to diminished effects of RNAi at elevated temperatures [61]. We tested whether cells episomally expressing Sir2, Sir2 N247A, or Sir2 $\Delta 1-129$ could complement for the silencing defect observed in sir $2 \Delta$ cells. Expression of WT Sir2 efficiently silenced the centromeric $\mathrm{ura}^{+}$reporter, allowing cells to grow on 5-FOA, which is toxic to cells expressing $\mathrm{ura}^{+}$. Sir2 N247A could also silence the reporter, as could Sir2 $\Delta 1-129$, but cells expressing the double mutant, Sir2 $\Delta 1-129$ N247A, were unable to silence the reporter (Figure 3-1C). This result suggests that both the enzymatic activity and the NTD contribute to the centromeric heterochromatin silencing activity of Sir.

To ensure that the silencing effects observed were not linked to overexpression of the proteins, we integrated constructs for Sir2-3x V5, Sir2 N247A-3x V5, and Sir2 $\Delta 1$ 129-3x V5 into the genomic $s i r 2^{+}$locus and monitored heterochromatin maintenance. To do this, total RNA was prepared from cells bearing the integrated Sir2 constructs in order to make cDNA. The maintenance of centromeric silencing was examined using qRT-PCR to monitor the levels of $d h$ centromeric transcripts from the otr which were normalized to adhl, a euchromatic control locus (Figure 3-1D). The results indicate that integrated sir2-3x V5 can fully complement for WT Sir2, with little transcript accumulation in 
A.

Sir2 Domains

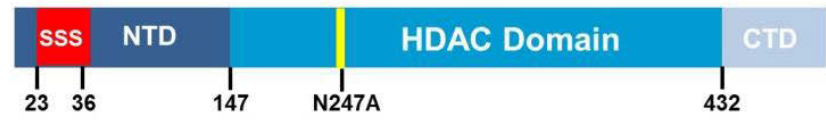

C.

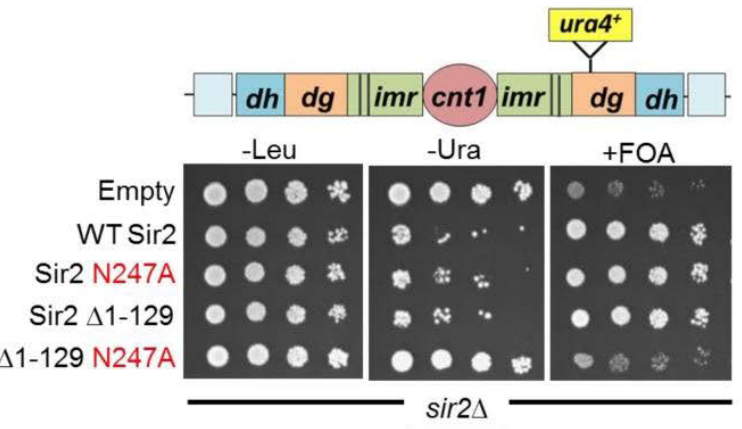

E.

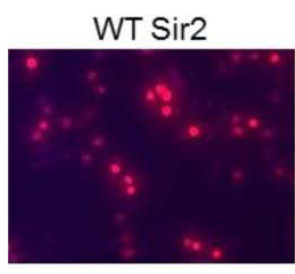

$\operatorname{Sir} 2 \Delta 1-20$

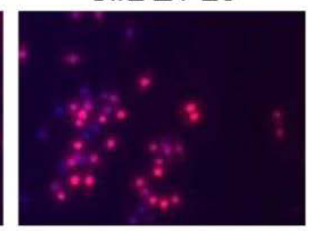

V5 IF in $\operatorname{sir} 2 \Delta^{E}$
B.

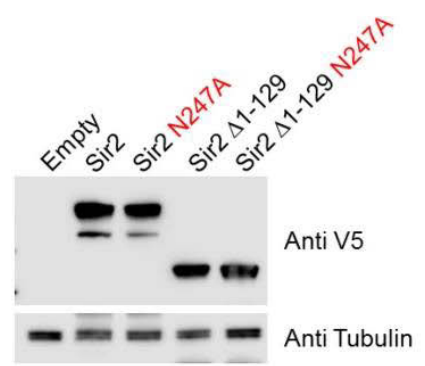

D.

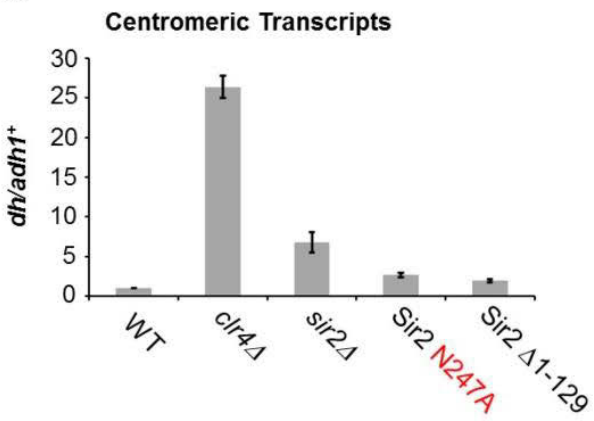

Figure 3-1. The NTD and HDAC activity of Sir2 are important in heterochromatin maintenance.

A. Schematic of fission yeast Sir2 domains which includes a large HDAC domain surrounded by the auxiliary NTD and CTD. The NTD is largely uncharacterized, containing a serine rich patch between aa23-36. The HDAC domain contains a conserved asparagine residue, N247, which is necessary for HDAC activity. B. Immunoblotting indicating equal expression of the Sir2-3x V5 constructs. C. Centromeric silencing assay monitoring silencing of an otr 1 R::ura $4^{+}$reporter gene in strains containing episomally expressed Sir2 and Sir2 N247A NTD deletion constructs by growth on PMG media containing 5-FOA. Both the HDAC activity and NTD of Sir2 are necessary for centromeric heterochromatin maintenance at $33^{\circ} \mathrm{C}$. D. Centromeric $d h$ transcripts measured by qRT-PCR from strains bearing integrated sir2 N247A and sir2 $41-129$. Integrated sir $2 N 247 A$ and $\operatorname{sir} 2 \Delta 1-129$ bearing strains show only a minor increase in centromeric $d h$ transcripts. E. Immunofluorescent analysis of cells transformed with episomal Sir2-3x V5, Sir2 $\Delta 1-20-3 x$ V5, and Sir $\Delta 1-129-3 x$ V5 constructs to examine Sir2 cellular localization. Deletion Sir2 residues 1-129 does not impact Sir2 nuclear localization. 
contrast to the $\operatorname{clr} 4 \Delta$ and $\operatorname{sir} 2 \Delta$ controls. The $\operatorname{sir} 2 \Delta$ strain exhibits slightly elevated levels of centromeric transcripts, while both sir2 N247A and sir2 $41-129$ exhibit only a slight elevation of centromeric transcripts. This data corroborates the data using our episomally expressed Sir2 constructs, suggesting that both the NTD and HDAC activity contribute to the role of Sir2 in heterochromatic silencing.

\section{Sir2 $\Delta$ 1-129 Can Localize to the Nucleus}

Since the NTD of Sir2 contributes to the maintenance of centromeric heterochromatin, it is possible that Sir2 1-129 contributes to Sir2 nuclear localization. Although the predicted nuclear localization signal (NLS) of Sir2 lies further downstream in the sequence, we wanted to rule out any role of the NTD in nuclear localization. We utilized immunofluorescence to visualize the localization of the episomally expressed Sir2 constructs in sir2 $\Delta$ cells by monitoring the $3 x$ V5 tag (Figure 3-1E). The immunofluorescence analysis indicates that $\operatorname{Sir} 2 \Delta 1-129$ can localize to the nucleus similar to WT Sir2. This shows the defect in complementation by Sir2 $\Delta 1-129$ is not caused by exclusion from the nucleus.

\section{Role of the Sir2 NTD in Heterochromatin Establishment}

Since the main function of Sir2 at the centromere is in the establishment of heterochromatin, we next tested the role of the Sir2 NTD in establishment. To quickly analyze the role of the Sir2 NTD in centromeric heterochromatin establishment, we generated a strain to function as a Sir2 establishment reporter strain $\left(\operatorname{sir} 2 \Delta^{E}\right)$. To generate this strain, a $\operatorname{sir} 2 \Delta$ strain was crossed with a $\operatorname{cl} 4 \Delta$ strain to produce a $\operatorname{sir} 2 \Delta \operatorname{clr} 4 \Delta$ strain. clr $4^{+}$was then reintegrated into its endogenous locus and confirmed by southern analysis to be present as a single copy (Figure 3-2A). This $\operatorname{sir} 2 \Delta^{E}$ strain cannot establish de novo silencing of the centromeric otr $1 R:: u r a 4^{+}$reporter when transformed with an empty vector, but cells transformed with episomal WT Sir2 efficiently establish heterochromatin de novo, shown by the growth on FOA containing medium (Figure 3-2B). Episomally expressed Sir2 N247A shows partial establishment of heterochromatin compared to WT Sir2, confirming previous reports [83]. Interestingly, Sir2 $\Delta 1-129$ transformants show similar ability to establish silencing as WT Sir2 transformants, but Sir2 $\Delta 1-129$ N247A transformed cells were completely deficient for the establishment of centromeric heterochromatin, mirroring the sir $2 \Delta$ phenotype. These results indicate that both the NTD and catalytic activity of Sir2 are required to properly establish centromeric heterochromatin. Due to the combinatorial effect of the NTD deletion with the N247A catalytic mutant, all subsequent work examining the role of the Sir2 NTD in centromeric heterochromatin establishment was performed in the context of N247A.

Sequence analysis of the Sir2 NTD revealed a highly serine rich region located between residues 23 and 36, in which 9 out of the 14 residues are serine (Figure 3-3A). Several of the serine residues within this region are conserved with another member of the Schizosaccharomyces genus, Schizosaccharomyces octosporus, which like fission 
A.

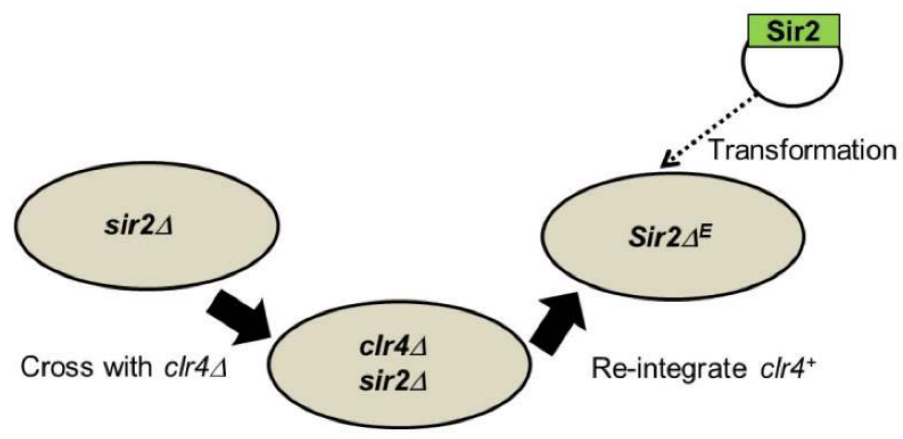

B.

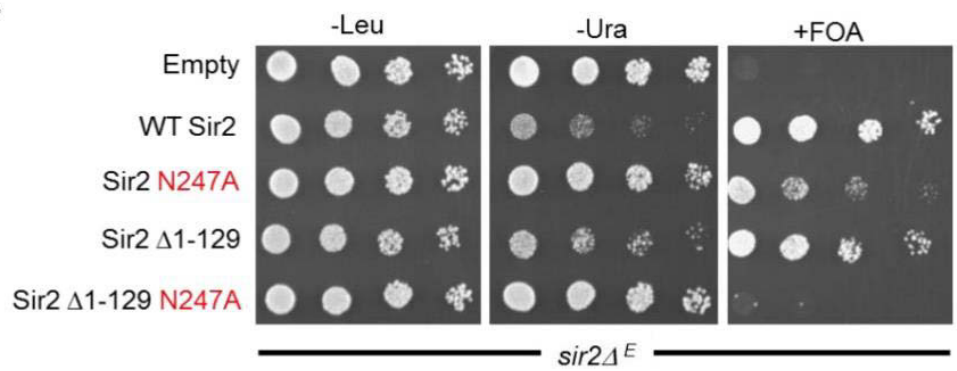

Figure 3-2. The NTD and HDAC activity of Sir2 are important for heterochromatin establishment.

A. Diagram of the centromeric heterochromatin establishment assay using episomally Sir 2 constructs. An establishment was created by first creating a sir $2 \Delta \operatorname{clr} 4 \Delta$ double mutant strain containing an otr $1 R::$ ura $^{+}$reporter gene in which $\mathrm{clr} 4^{+}$was then reintegrated sole copy into the genomic locus, creating the sir $2 \Delta^{E}$ establishment strain. The $\operatorname{sir} 2 \Delta^{E}$ strain was then transformed with the episomal Sir2 constructs to monitor de novo centromeric heterochromatin establishment. B. Establishment assay to monitor de novo centromeric heterochromatin formation using an otr $1 R:: \mathrm{ura}^{+}$reporter gene by examining growth on media containing 5-FOA. Cells expressing Sir2 N247A or Sir2 $\Delta 1$ 129 can establish some centromeric heterochromatin. In contrast, cells expressing Sir2 $\Delta 1-129 \mathrm{~N} 247 \mathrm{~A}$ are defective for the establishment of centromeric heterochromatin. 
A.

\begin{tabular}{|c|c|c|c|}
\hline & & $\begin{array}{c}\text { serine rich patch } \\
\text { aa23-36 }\end{array}$ & \\
\hline pombe & -MASNPLDNNMPTT PVEEKI PVI & ASYSPSSSGSSSGASLLVDIMCGSKETEDEEVDSDEWD & 59 \\
\hline \multirow[t]{2}{*}{ octosporus } & MSFSPVEDLHSTPKEVNSEGKDI & DIETSSTSSISSSASLLVDIMCGSKDAEDSEAESDEWD & 60 \\
\hline & $\star \quad * \quad: \ldots *$ * $:$. & 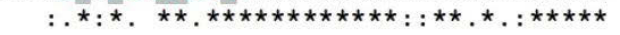 & \\
\hline pombe & KPETENISDLDERSEMVRYLRAS & SGYAKFLEKYLIEEELPVRSILKKLGINLPSALEEFED & 119 \\
\hline \multirow[t]{2}{*}{ octosporus } & RPVIDDHEVIKQREEILKSLRII & LGYPAFLQKHVVEQQIP IRLILKKL GISLPTALDEFED & 120 \\
\hline & 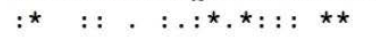 & 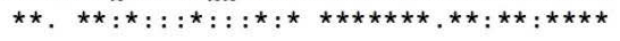 & \\
\hline ombe & IDLIPLIKEV 129 & & \\
\hline ctosporus & LDILPLIKEV 130 & & \\
\hline
\end{tabular}

B.

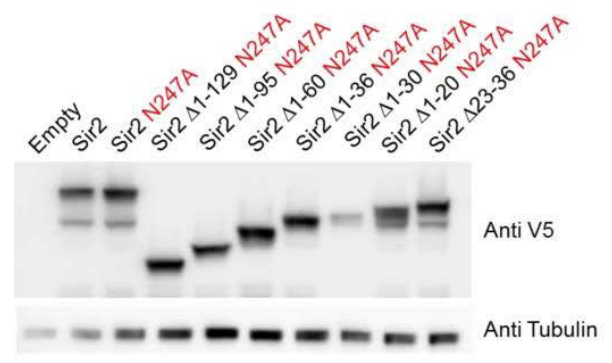

C.

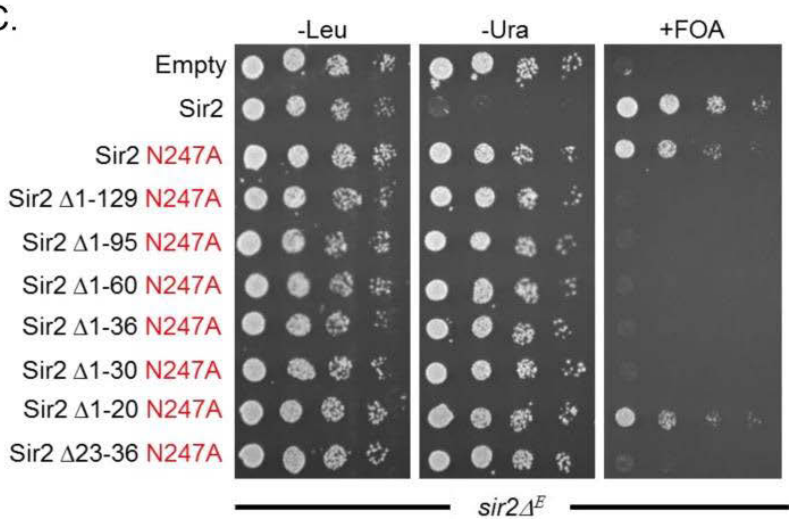

Figure 3-3. The serine rich patch of the Sir2 NTD is important for centromeric heterochromatin establishment.

A. Sequence alignment of the fission yeast Sir2 NTD with S. octosporus. The serine rich patch within the Sir2 NTD lies between residues 23 and 36. Several of the serine residues within this patch are conserved with the closely related $S$. octosporus. B. Immunoblotting to examine the expression of the Sir2 N247A NTD truncations. All Sir2 truncations with the exception of Sir2 $\Delta$ 1-30 show similar protein expression levels. C. Establishment assay to monitor de novo heterochromatin establishment in $\operatorname{sir} 2 \Delta^{E}$ cells transformed with the episomal Sir2 N247A NTD truncations by monitoring silencing of an otr1R::ura ${ }^{+}$ reporter gene using media containing 5-FOA. Deletion of the first 36 residues of the NTD in the HDAC null background (Sir2 $\Delta 1-36$ N247A) causes total loss of de novo centromeric heterochromatin establishment, while leaving the entire serine rich patch intact (Sir2 $\Delta 1-20$ N247A) allows for heterochromatin establishment at levels similar to Sir2 N247A. Deleting the entire serine rich patch (Sir2 $\Delta 23-36$ N247A) leads to a complete loss of centromeric heterochromatin establishment. 
yeast has highly repetitive regions around the centromere where heterochromatin is thought to form [202]. We termed this region of the Sir2 NTD between residues 23 and 36 the serine rich patch. Due to the unusual nature of this region, we hypothesized it may be involved in the role of the Sir2 NTD in heterochromatin establishment.

In order to test whether the serine rich patch of Sir2 (aa23-36) is important for pericentromeric heterochromatin establishment, a series of truncations were constructed in the Sir2 N247A vector, deleting residues 1-95, 1-60, 1-30, 1-20, and 23-36. The truncations were made in the Sir2 N247A plasmid since the combined loss of HDAC activity with the NTD deletion caused the most severe establishment defect. All the truncations were expressed similarly with the exception of Sir2 $\Delta 1-30$ N247A, which showed slightly lower levels than WT Sir2 (Figure 3-3B). The plasmids were tested for their ability to confer establishment of centromeric heterochromatin in the $\operatorname{sir} 2 \Delta^{E}$ strain background (Figure 3-3C). While expression of Sir2 N247A shows partial heterochromatin establishment, deletion of just the first 36 residues of Sir2 N247A (Sir2 $\Delta 1-36$ N247A) causes a complete failure to establish heterochromatin similar to the Sir2 $\Delta 1-129$ N247A construct. Sir2 $\Delta 1-30$ N247A, which cuts the serine rich patch in half also shows a lack of heterochromatin establishment. In contrast, Sir2 $\Delta 1-20$ N247A in which none of the serine rich patch is deleted can establish centromeric heterochromatin similar to Sir2 N247A. When the entire serine rich patch is specifically deleted in the Sir2 N247A background, Sir2 $\Delta 23-36$ N247A, no establishment was observed similar to Sir2 $\Delta 1-129$ N247A. Taken together, these results indicate the serine rich patch within the NTD of Sir2, together with HDAC function, is important for centromeric heterochromatin establishment.

To further narrow down the region of the Sir2 serine rich patch important for establishment in conjunction with HDAC activity, we constructed additional truncations within this region: $\operatorname{Sir} 2 \Delta 1-27$, Sir2 $\Delta 23-30$, and $\operatorname{Sir} 2 \Delta 27-30$ (Figure 3-4A). Again, these constructs were made in the Sir2 N247A background and transformed into the $\operatorname{sir} 2 \Delta^{E}$ strain. Immunoblotting indicated all the proteins were stably expressed at similar levels to WT Sir2 (Figure 3-4B). Both Sir2 $\Delta 1-27$ N247A and Sir2 $\Delta 27-30$ N247A could establish centromeric heterochromatin similar to Sir2 N247A (Figure 3-4C). In contrast, Sir2 $\Delta 23-$ 30 N247A was unable to establish centromeric heterochromatin. This data suggests that the SSSG repeats at residues 27 and 31 may function with the SYSP residues at aa23-26 within the NTD of Sir2 to establish centromeric heterochromatin in conjunction with its HDAC activity.

\section{Deletion of the Sir2 NTD Does Not Impact HDAC Activity}

Deletion of residues 1-129 of Sir2 causes defects in heterochromatin establishment in the $\operatorname{sir} 2 \Delta^{E}$ strain when assessed in the context of the HDAC defective N247A mutant. Similarly, deletion of residues Sir2 $\Delta 1-129$ N247A causes defects in the

maintenance of centromeric heterochromatin when tested at higher temperatures. Because both situations rely on HDAC function at centromeres, we needed to rule out the possibility that the NTD of Sir2 may modulate its HDAC activity. It has previously been 
A.

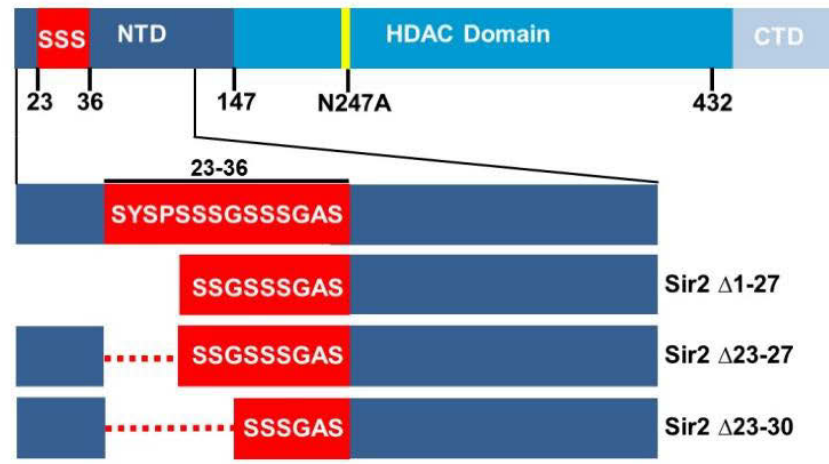

B.

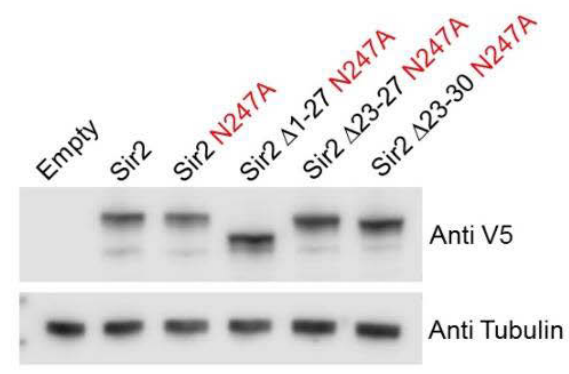

C.

\begin{tabular}{|c|c|c|c|c|c|c|}
\hline & -Leu & & -Ura & & & OA \\
\hline Empty & 0000 & 0 & 0 & 5 & $\cdots$ & \\
\hline Sir2 & ○○०\% & - & 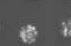 & $=$ & - • & - * \\
\hline Sir2 N247A & 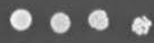 & - & 0 & 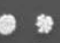 & - & is \\
\hline r $2 \Delta 1-129$ N247A & 000 : & 0 & 0 & to & . & \\
\hline Sir2 $\Delta 1-27 N 247 A$ & 000 & & 0 & 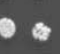 & - & $\theta$ \\
\hline $\operatorname{Sir} 2 \Delta 1-27$ N247A & 000 & 0 & 0 & 0 & 0 & $\approx$ \\
\hline Sir2 $\triangle 27-30$ N247A & 000 & 0 & 0 & 3 & 0 & 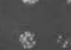 \\
\hline Sir2 $\triangle 27-30$ N247A & 000 \% & 0 & 0 & 0 : & 0 & 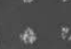 \\
\hline Sir2 $\triangle 23-30$ N247A & 000 & 0 & 0 & $=$ & & \\
\hline ir $\triangle 23-30$ N247A & $\bullet \bullet \bullet \bullet$ & 0 & 0 & 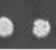 & & \\
\hline
\end{tabular}

Figure 3-4. The SSSG repeats are important for de novo centromeric heterochromatin establishment.

A. Schematic showing the Sir2 N247A truncation constructs. Three truncations in the first 30 residues of Sir2 N247A were constructed: $\Delta 1-27, \Delta 27-30$, and $\Delta 23-30$. B. Immunoblotting showing that Sir2 $\Delta 1-27, \Delta 27-30$, and $\Delta 23-30$ N247A are stably expressed. C. Establishment assay to monitor de novo centromeric heterochromatin establishment in $\operatorname{sir} 2 \Delta^{E}$ transformed with the episomal Sir2 N247A truncations within residues 1-30 by monitoring the silencing of an otr $1 R:: u r a^{+}$reporter gene. Deletion of the entire 23-30 region of the Sir2 N247A NTD (Sir2 $\Delta 23-30$ N247A) causes a complete loss of centromeric heterochromatin establishment, while partial deletion of this patch allows for the establishment of centromeric heterochromatin at levels similar to cells transformed with episomal Sir2 N247A. 
shown that the Sir2 N247A mutant is catalytically dead both in vitro using recombinant protein in an HDAC assay and in vivo by monitoring the effects at telomeres where Sir2 is required for heterochromatin maintenance [83]. However, the possibility remained of some residual HDAC activity in the Sir2 N247A mutant which may be negatively affected by the Sir2 NTD deletion. This could lead to the observed maintenance and establishment defects when the Sir2 NTD deletion is combined with the N247A HDAC mutant.

In order to address this concern, we purified recombinant GST tagged Sir2 from E. coli to test the HDAC activity of Sir2 $\Delta 1-129$. We also purified Sir2 $\Delta 1-137$ to identify how much of the NTD is required for HDAC activity. All the purified recombinant proteins appeared stable and migrated to the correct size on a coomassie stained gel (Figure 3-5A). To test HDAC activity, we utilized the Fluor De Lys ${ }^{\circledR}$ Green HDAC fluorometric assay from Enzo Life Sciences. In this assay, a generic fluorogenic acetylated lysine substrate is incubated with recombinant $\mathrm{Sir} 2$ proteins and $\mathrm{NAD}^{+}$ (Figure 3-5B). As Sir2 deacetylates the substrate, a fluorophore is generated and fluorescence can be measured after the addition of a developer reagent. This fluorescent measurement was then converted to percentage of WT Sir2 KDAC (lysine deacetylase) activity. Our lab has previously shown that Sir2 N247A has no KDAC activity in this assay [83]. In contrast, recombinant GST-Sir2 $\Delta 1-129$ has KDAC activity at levels comparable to GST-WT Sir2 (Figure 3-5C). The recombinant GST-Sir2 $\Delta 1-137$ showed no KDAC activity, indicating that residues 1-129 demarcates the boundary beyond which mutations impinge on KDAC activity [unpublished communication from Ben Alper (Sacred Heart University) on 03/21/2016]. This data suggests that deleting residues 1-129 from the NTD of Sir2 does not impact HDAC activity in vitro.

Although our in vitro HDAC assay suggested Sir2 $\Delta 1-129$ is fully HDAC competent, it is possible that deacetylation of natural targets in vivo may be affected by the removal of the Sir2 NTD. To address this concern, we utilized ultra-high performance liquid chromatography tandem mass spectrometry (UPLC-MS/MS) to examine histone $\mathrm{H} 3$ and $\mathrm{H} 4$ acetylation in strains expressing genomic Sir2-3x V5, Sir2 N247A-3x V5, and Sir2 $\Delta 1-129-3 \mathrm{x}$ V5.

Histone purification from fission yeast is difficult since we cannot isolate pure nuclei efficiently. Instead, we isolated a chromatin enriched fraction which was then acid extracted and further purified by binding to Bio-Rex $70^{\mathrm{TM}}$ cation exchange resin to obtain material pure enough for mass spectrometry (Figure 3-6A, B). Mass spectrometry of semi-purified histones was performed by Ryan Henry and Andy Andrews (FCCC), and acetylation levels were quantified for lysine residues within the N-terminal tails of histones $\mathrm{H} 3$ and $\mathrm{H} 4$ (Figure 3-6C) [unpublished communication from Andy Andrews and Ryan Henry on 12/21/16]. For this experiment, histones were enriched from triplicate biological replicates for each strain. The MS/MS technique utilizes propionylation of the sample before tryptic digestion. This chemically modifies unmodified or monomethylated lysine residues and blocks trypsin cleavage, allowing for the generation of larger more reproducible peptide fragments from the lysine rich N-terminal histone tails [200]. Selected reaction monitoring and quantitation of MS/MS in which synthetic peptides 
A.

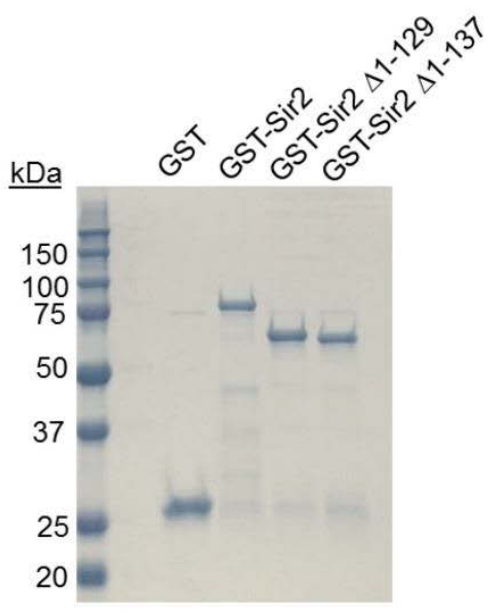

C.

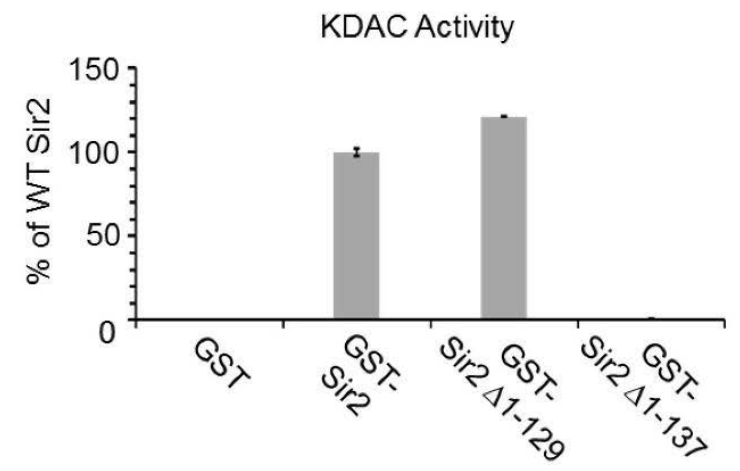

B.

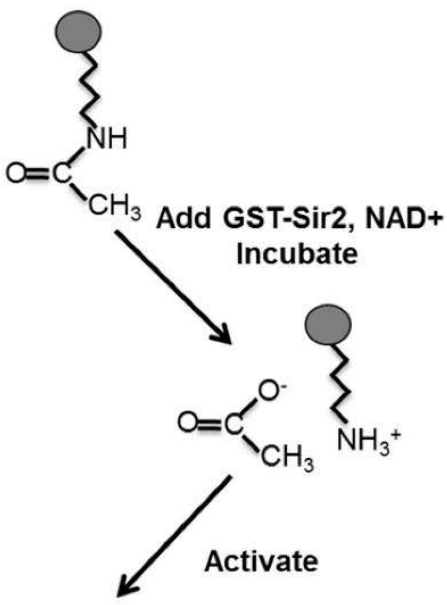

Ex:485 nm

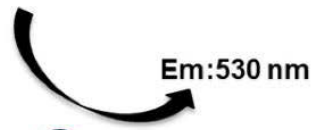

Figure 3-5. Deletion of residues 1-129 of Sir2 does not impact HDAC activity in vitro.

A. Coomassie stained gel of recombinant GST-Sir2 constructs purified from E. coli. GST-Sir2, GST Sir2 $\Delta 1-129$, and GST Sir2 $\Delta 1-137$ are stable and correspond to the correct molecular weights. B. Schematic of the Fluor de Lys ${ }^{\circledR}$ Green HDAC assay protocol. In this assay, recombinant Sir2 is incubated with $\mathrm{NAD}^{+}$and an acetylated lysine substrate. Deacetylation of the substrate and addition of the developer reagent produces a fluorophore, allowing fluorescence to be measured at $530 \mathrm{~nm}$ and converted to a readout of KDAC activity. C. KDAC measurements using the Fluor de Lys ${ }^{\circledR}$ assay and the recombinant GST Sir2 constructs. The fluorescence readout created by deacetylation of the substrate was converted to KDAC activity and graphed as a percentage of WT recombinant Sir2 activity. Recombinant Sir2 $\Delta 1-129$ has KDAC activity comparable to WT, while recombinant Sir2 $\Delta$ 1-137 exhibits no KDAC activity.

Source: The Fluor de Lys ${ }^{\circledR}$ assay in panel C was performed in collaboration with Ben Alper [unpublished communication on 03/21/2016]. 
A.

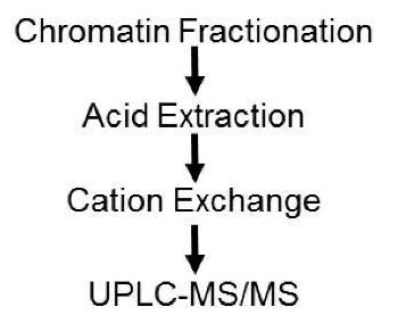

B.

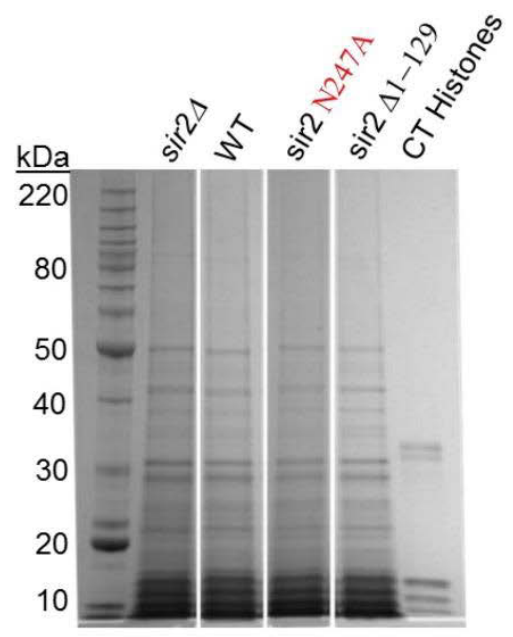

C.
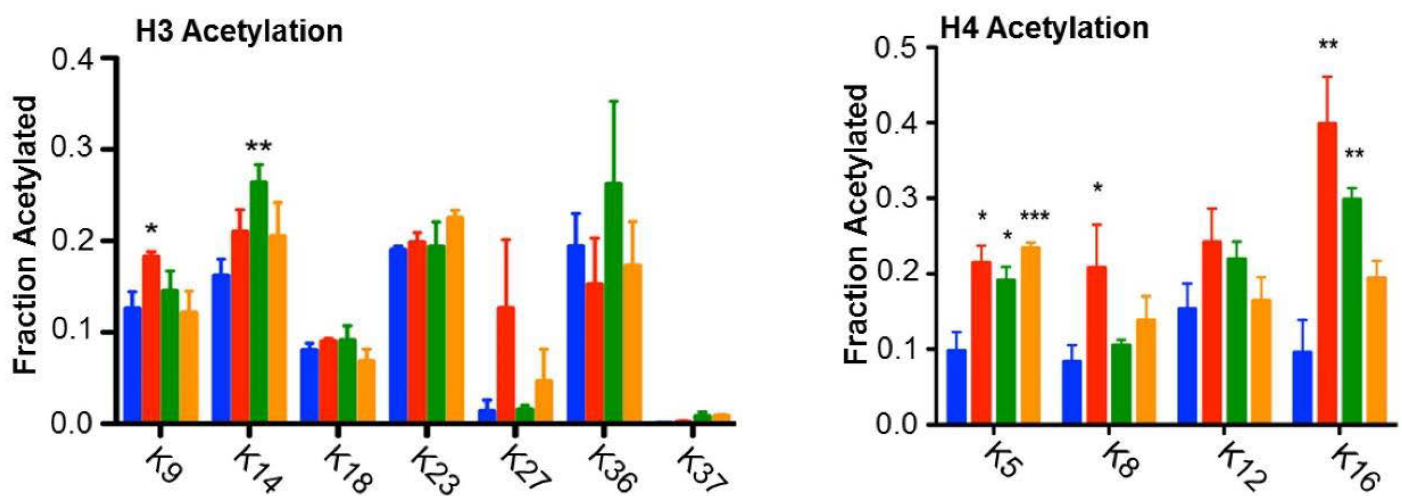

$\begin{array}{lll}\text { WT Sir2 } & * & \mathrm{P}<.05 \\ \text { sir2 } & * * & \mathrm{P}<.01 \\ \text { Sir2 N247A } & * * * & \mathrm{P}<.001 \\ \text { Sir2 } \Delta 1-129 & & \end{array}$

Figure 3-6. Deletion of residues 1-129 of Sir2 does not impact HDAC activity in vivo.

A. Schematic showing histone purification from fission yeast. We first isolate a chromatin fraction which is subjected to acid extraction and cation exchange to obtain material pure enough for targeted UPLC-MS/MS. B. Coomassie stained gel of acid extracted histones used for MS/MS (CT - calf thymus histones). C. Targeted UPLC MS/MS analysis of histones purified from WT, sir2 $\Delta$, sir2 N247A, and sir2 $41-129$ strains. The sir2 N247A strain shows increased H3K9ac and H4K16ac, while the sir2 $\triangle$ strain shows increased H4K16ac. The sir2 $41-129$ strain has levels of H3K9 and H4K16ac similar to the WT control.

Source: MS/MS shown in panel C was performed by Ryan Henry and Andy Andrews on histones purified in our lab [unpublished communication on 12/21/2016]. 
representing the actual histone peptides are used to optimize detection of the actual peptides allows for targeting of specific peptides of interest in a high throughput manner [196].

The targeted MS/MS analysis showed a significant increase in H3K9ac in the $\operatorname{sir} 2 \Delta$ strain as indicated by the p-value $<.05$ (Figure 3-6C). In contrast, levels of H3K9ac in both the sir2 N247A strain and the sir2 $\Delta 1-129$ strain had levels similar to the WT control. Conversely, only the sir2 N247A strain had a significant increase in H3K14ac, with a p-value $<.01$. It is likely that more samples will be needed to elucidate small effects which may be occurring at these sites. We also observed a significant increase in H4K16ac, another Sir2 target, in both the sir2 $\Delta$ and $\operatorname{sir} 2 N 247 A$ strains. In contrast, the only significant increase in acetylation observed in the sir2 $\Delta 1-129$ strain was at H4K5, which was also increased in the sir2 $\Delta$ and $\operatorname{sir} 2 N 247 A$ strains. Taken together, this data suggests that Sir2 $\Delta 1-129$ can efficiently deacetylate known Sir2 targets in vivo, indicating that deletion of residues 1-129 does not significantly impact HDAC activity in contrast to the Sir2 N247A mutant.

\section{MS/MS of Sir2 and Sir $\Delta 1-129$}

To date, no interaction partners for fission yeast Sir2 have been identified. This has complicated efforts to understand how Sir2 is recruited to chromatin since most HDACs act in complexes which aid in their targeting to specific regions of the cell. These interactions often occur through auxiliary domains outside of the enzymatic HDAC core. It is possible that the NTD of Sir2 acts as a scaffold to bind proteins which target Sir2 to heterochromatic areas. This could explain the importance of the Sir2 NTD in the de novo silencing events of centromeric heterochromatin establishment.

In order to identify potential Sir2 interactors, we purified episomally expressed full length Sir2-3x V5 and Sir2 $\Delta 1-129-3 x$ V5. This method allowed us to interrogate whether full length Sir2 can interact with proteins that the truncated Sir2 $\Delta 1-129$ cannot. This would give preliminary evidence of any potential interactions mediated by the Sir2 NTD. The Sir2 constructs were purified using the 3x V5 tag and eluted using V5 peptide in two elutions. The efficiency of the IP was examined by running $2 \%$ of the purified proteins on a gel which was coomassie stained (Figure 3-7A). This purified material was then sent for LC-MS/MS by Alan Tackett (UAMS).

The results of the MS/MS did not provide significant insight into potential Sir2 interactors (Table A-4) [unpublished communication from Alan Tackett on 11/23/2015]. No stoichiometric interactors were identified, making it difficult to suggest any proteins that Sir2 may be in complex with. Also, only minor differences in interactors were observed between full length Sir2 and Sir2 $\Delta 1-129$, suggesting the Sir2 NTD does not serve as a scaffold for protein interaction. Previous MS/MS of Sir2 identified the fission yeast 14-3-3 like proteins Rad24 and Rad25 as potential interactors of Sir2. Deletion of $\operatorname{Rad} 24$ does not have an establishment defect, making it unlikely that it is required for the role of Sir2 in heterochromatin establishment, although Rad25 could be compensating for 
Figure 3-7. Phosphorylation of the Sir2 serine rich patch does not impact centromeric heterochromatin establishment.

A. Coomassie gel of episomal Sir2-3x V5 purified from fission yeast. Full length Sir2-3x V5 and Sir2 $\Delta 1-129-3 x$ V5 were purified from fission yeast using V5 agarose and $2 \%$ of the purified material was run on a gel for coomassie staining. B. LC-MS/MS analysis indicating phosphorylation of the Sir2 serine rich patch. MS/MS analysis for Sir2 PTM indicates that S23, 25, and 27 of the serine rich patch are phosphorylated along with T13 of the NTD. C. Establishment assay to monitor de novo centromeric heterochromatin establishment in the sir2 $\Delta^{E}$ strain transformed with episomal Sir2 N247A phosphorylation (aspartic acid; D) or dephosphorylation mimetics (alanine; A) by monitoring silencing of an otrlR::ura ${ }^{+}$reporter gene. Phosphorylation or dephosphorylation mimetics of S23, 25, or 27 alone nor the combined phosphorylation or dephosphorylation mimetics do not impact the de novo establishment of centromeric heterochromatin when transformed into the $\operatorname{sir} 2 \Delta^{E}$ strain. D. Immunoblotting indicates all phosphorylation and dephosphorylation mimetics were expressed at equal levels except Sir2 T13D.

Source: MS/MS shown in panel B was performed by Alan Tackett on histones purified in our lab [unpublished communication on 10/01/2015]. 
A.

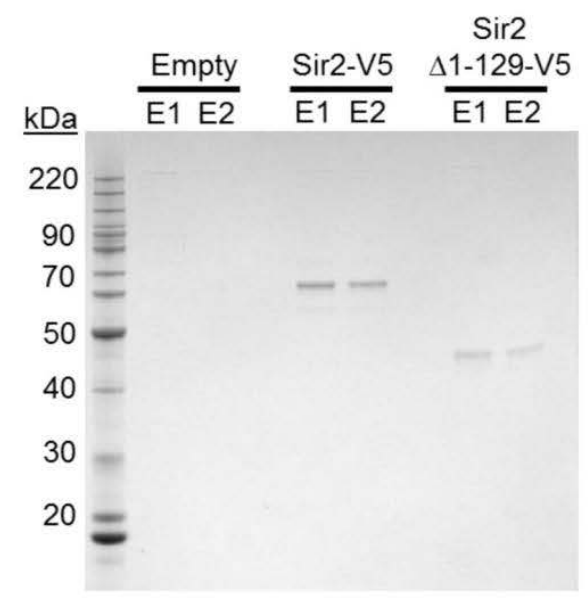

B.

Sir2 NTD

Phosphorylation

MASNPLDNNMPTTPVEEKIPVASYSPSSSGSSSGASLLVD

C.

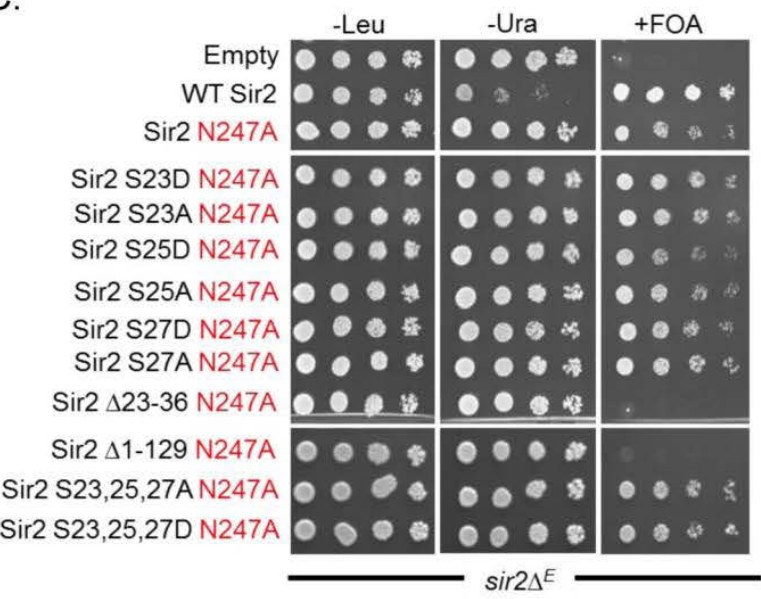

D.

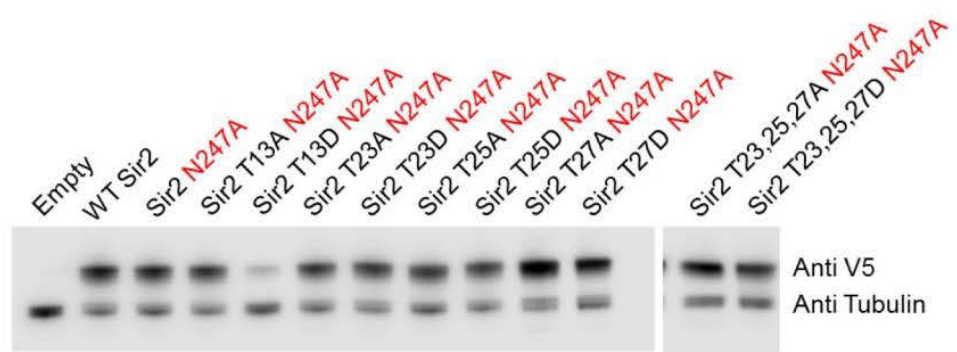


the $\mathrm{rad} 24 \Delta$ due to a high degree of similarity between the proteins [unpublished communication from Janet Partridge on 03/03/2017]. This cannot be fully tested since simultaneous deletion of both $\operatorname{Rad} 24$ and $\operatorname{Rad} 25$ is lethal in fission yeast [203]. Our current MS/MS also identified $\operatorname{Rad} 24$ and $\operatorname{Rad} 25$ as potential Sir2 interactors, but with less certainty due to high amounts of these proteins in the control purification from a $\operatorname{sir} 2 \Delta$ strain. It is possible that purifying overexpressed episomal Sir2 may obscure any true interactors, so further research and MS/MS will be required to resolve this issue.

\section{The Sir2 NTD Is Post-Translationally Modified}

Currently, MS/MS analysis of Sir2 has provided little insight into how the Sir2 NTD is contributing to heterochromatin establishment. Since the serine rich patch within the NTD of fission yeast Sir2 is important for its role in the establishment and maintenance of centromeric heterochromatin, we examined whether this region is a site of PTM. It is possible that PTM within this region may be important in the regulation or recruitment of Sir2 in vivo. This is supported by the fact that many sirtuins undergo PTM within their NTD and CTD which contributes to their regulation.

In order to determine whether Sir2 undergoes PTM of its NTD, we used Sir2 purified from fission yeast for MS/MS. Digestion conditions for Sir2 were optimized by Alan Tackett [unpublished communication from Alan Tackett on 06/26/2015]. This was necessary since previous peptide generation from the Sir2 NTD was poor when the standard trypsin cleavage was used. To resolve this issue, chymotrypsin cleavage conditions were optimized for the Sir2 NTD using bacterially expressed recombinant Sir2, allowing for greater than $80 \%$ sequence coverage of Sir2. Also, due to poor yields from previous purifications of endogenous Sir2, we decided to use overexpressed episomal Sir2-3x V5 purified as described above. LC-MS/MS analysis of this material was performed by Alan Tackett using the optimized chymotrypsin digestion conditions.

Two different types of MS/MS analysis, collision-induced dissociation (CID) and high-energy collisional dissociation (HCD) fragmentation, were utilized to detect PTMs in the Sir2 NTD (Table A-5) [unpublished communication from Alan Tackett on 10/01/2015]. Overall, greater than 80\% peptide coverage of Sir2 was achieved, including good coverage of the serine rich patch in the Sir2 NTD, although no specific protein interactors were identified. MS/MS using CID fragmentation indicated phosphorylation of T13 which has been previously shown (Figure 3-7B and Table A-5) [204]. We also observed novel phosphorylation of S23 and S25 within the serine rich patch using this method. The HCD fragmentation method also identified phosphorylation at T13 along with S23 and S25 phosphorylation. In addition, this method identified another novel phosphorylation site at S27 within the serine rich patch. Further MS/MS will be needed to confirm these results showing novel sites of phosphorylation on Sir2 at S23, 25 and 27 in vivo.

In order to test the importance of PTM of S23, 25, and 27, we mutated these residues individually to either an aspartic acid to mimic phosphorylation or an alanine to 
mimic dephosphorylation of the serine residue in the episomally expressed Sir2 N247A construct. We then tested the effects of these mutations in the sir $2 \Delta^{E}$ establishment reporter strain using a centromeric silencing assay (Figure 3-7C). The results indicate that mutating each serine residue individually to an A or D showed no centromeric heterochromatin establishment defect in comparison to the Sir2 $\Delta 23-36$ N247A or Sir2 $\Delta 1-129$ N247A control strains and that all mutants were expressed similarly (Figure 3-7D). We next combined the mutations at S23, 25, and 27, creating Sir2 23, 25, 27A/D mutants in the N247A background to test the combinatorial effect of the mutations. When tested by silencing assay, no significant discernible effects on centromeric heterochromatin establishment were observed. Although no effects on heterochromatin establishment were observed from mutating the serine residues, it is possible that we did not create the correct combination of phosphorylated/dephosphorylated mimetics to reproduce the defects observed when the serine patch is absent.

\section{The Sir2 NTD Is Important for Chromatin Association}

It is currently unknown how Sir2 is recruited to its targets since no Sir2 containing complex has been identified in fission yeast. This severely complicates efforts to understand the basic mechanisms underlying how Sir2 performs its role in heterochromatin maintenance and establishment. Since we were unsuccessful in identifying potential interactors of Sir2, we chose to examine whether the NTD itself is necessary for the recruitment of Sir2 to heterochromatic regions.

ChIP assays using endogenous Sir2 have largely been unsuccessful, so we again utilized the episomally tagged Sir2-3x V5 constructs to examine chromatin association. We first tried to ChIP Sir2-3x V5 using protein G agarose beads, but this method led to little enrichment of Sir2 on chromatin. In order to improve the assay, protein $\mathrm{G}$ Dynabeads were used, which improved our ability to detect Sir2 enrichment on chromatin by 5-fold. In order to eliminate complexity, ChIP assays were performed in a simple sir $2 \Delta$ strain background using truncations in WT Sir2 rather than attempting ChIP in the $\operatorname{sir} 2 \Delta^{E}$ strain using the Sir2 N247A background. Although this experiment was performed as a ChIP, we were unable to monitor localization of Sir2 to a specific locus, but instead monitored general association with chromatin. In sir $2 \Delta$ cells, Sir $2 \Delta 1-20$ shows similar chromatin enrichment to WT Sir2 when examining $d h$ association using qRT-PCR (Figure 3-8A). In contrast, Sir2 $\Delta 1$-30 shows lower chromatin enrichment compared to WT Sir2. Further truncations of the Sir2 NTD decreased the ability of Sir2 to associate with chromatin, as shown by the Sir2 $\Delta 1-60, \Delta 1-95$, and $\Delta 1-129$. Interestingly, Sir2 N247A is severely limited in its ability to associate with chromatin. These results suggest that both the NTD and HDAC activity are necessary for Sir2 to properly associate with chromatin.

In order to address the loss of chromatin association exhibited by the Sir2 NTD truncations, we examined whether the Sir2 NTD can directly associate with nucleic acid. Many components of heterochromatin assembly can directly interact with nucleic acid though conserved domains which aid in recruitment to heterochromatic loci. Although 
A.

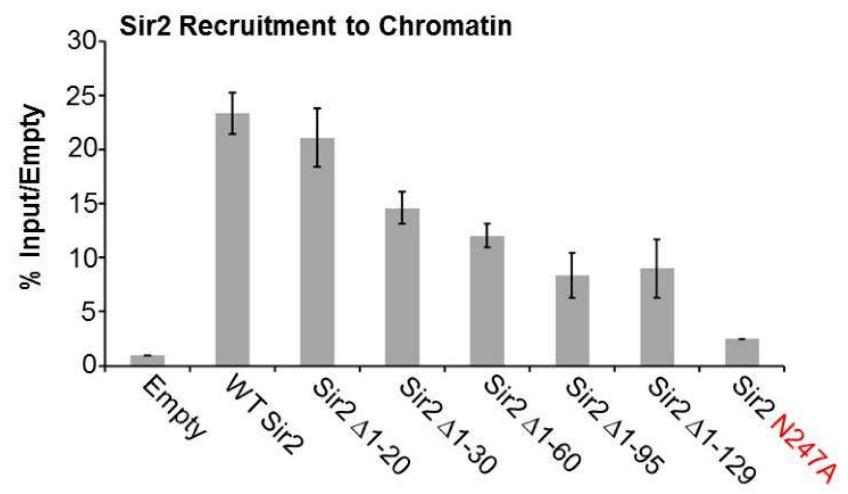

B.

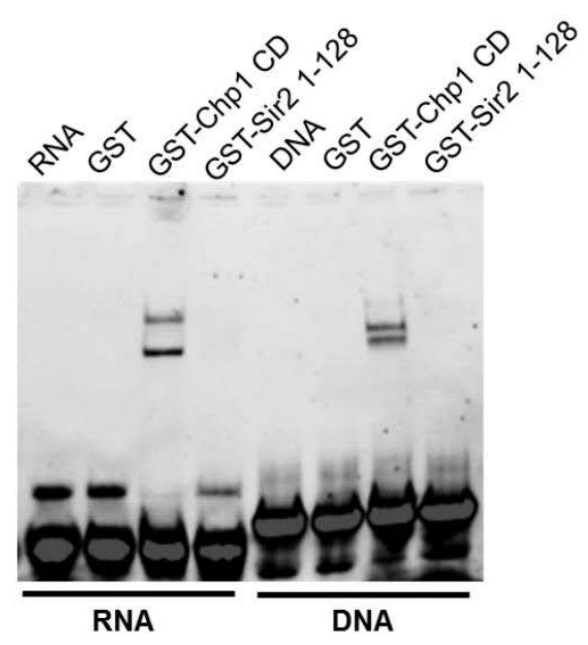

Figure 3-8. The Sir2 NTD and HDAC activity are necessary for proper recruitment of Sir2 to chromatin.

A. ChIP showing general chromatin association of the episomal Sir2 truncations in a $\operatorname{sir} 2 \Delta$ background. While both WT Sir2 and Sir2 $\Delta 1-20$ associate with chromatin at similar levels, further truncation of the Sir2 NTD causes reductions in the ability of Sir2 to associate with chromatin. The Sir2 N247A mutant is severely defective in chromatin association. qRT-PCR for this ChIP assay analyzed the association of Sir2 with $d h$ centromeric transcripts, but a similar pattern was observed at all loci tested. B. EMSA testing the interaction of recombinant GST-Sir2 1-128 with fluorescently labeled nucleic acid. While the Chp1 CD control can interact with ssRNA and dsDNA, recombinant Sir2 1-128 cannot interact with ssRNA or dsDNA. 
Sir2 does not share high conservation with nucleic acid binding proteins, we wanted to test the possibility that the NTD can associate with nucleic acid. To do this, an EMSA was performed using fluorescently labeled ssRNA and dsDNA (Figure 3-8B).

Recombinant GST-Chp1 CD was used as a positive control since it was previously shown to bind both RNA and DNA which aids in its chromatin recruitment [198]. In this assay, we tested the ability of recombinant GST-Sir2 residues 1-128 purified from E. coli to bind to either dsDNA or ssRNA. In contrast to the Chp1 CD control which binds nucleic acid, the Sir2 1-128 fragment cannot bind to either ssDNA or ssRNA. This indicates that direct nucleic acid binding by the Sir2 NTD is not responsible for the recruitment of Sir2 to chromatin, suggesting the recruitment of Sir2 is more complex. 


\section{CHAPTER 4. NOVEL TRANSCRIPTIONAL CONTROL AND TARGETS OF SHREC*}

\section{The Sir2 NTD Is Important for Redundancy with CIr3}

Previous research has shown the HDACs Sir2 and Clr3 act redundantly in the maintenance of centromeric heterochromatin, with $\operatorname{sir} 2 \Delta$ or $\operatorname{clr} 3 \Delta$ alone having little impact. In contrast, sir $2 \Delta$ clr $3 \Delta$ double mutant cells exhibit a complete loss of centromeric heterochromatin maintenance [83]. To further investigate the role of Sir2 in heterochromatin maintenance, we introduced episomally expressed Sir2 constructs into the $\operatorname{sir} 2 \Delta \operatorname{clr} 3 \Delta$ double mutant strain to determine if Sir2 $\Delta 1-129$ can complement for the redundant role of Sir2 in the absence of Clr3. Centromeric silencing was monitored by qRT-PCR to measure the level of $d h$ transcripts from the centromeric otr which were normalized to euchromatic $a d h 1$ transcripts (Figure 4-1). The $\operatorname{sir} 2 \Delta \operatorname{clr} 3 \Delta$ strain exhibits high levels of centromeric transcripts as previously described [83]. Episomally expressed WT Sir2 can complement for Sir2 function, bringing $d h$ transcript levels back to those observed in clr3 $\Delta$ cells. In contrast, neither Sir2 N247A nor Sir2 $\Delta 1-129$ can complement for the maintenance of pericentromeric heterochromatin in the $\operatorname{sir} 2 \Delta \operatorname{clr} 3 \Delta$ strain. This result indicates that both the NTD and HDAC activities of Sir2 are important for the redundant role of $\mathrm{Sir} 2$ with $\mathrm{Clr} 3$ in centromeric heterochromatin maintenance.

\section{Separation of Function in Global Transcriptional Regulation by SHREC Components}

To better understand the role of $\mathrm{Clr} 3$ in transcriptional regulation, we examined whether different modules within SHREC, of which Clr3 is a member, can function independently of one another. Previous evidence has suggested that SHREC components may function in independent modules since deletion of specific SHREC members shows variation in nucleosome occupancy at distinct loci depending on which components is removed [102]. To examine this possibility, RNA-seq was used to look for global transcriptional changes associated with the deletion of individual SHREC components (Figure 4-2A). Our RNA-seq analysis utilized triplicate biological replicates from individual cultures and applied a false discovery rate of $5 \%$ and a 1.5 -fold change cutoff to identify genes transcriptionally regulated by these components.

Our analysis of SHREC components using unsupervised hierarchical clustering (UHC) of the 1,000 most variable genes based on the median absolute deviation (MAD)

\footnotetext{
* Portions of Chapter 4 modified with permission from Elsevier Inc. Job, G., C. Brugger, T. Xu, B.R. Lowe, Y. Pfister, C. Qu, S. Shanker, J.I. Banos Sanz, J.F. Partridge, and T. Schalch, SHREC Silences Heterochromatin via Distinct Remodeling and Deacetylation Modules. Mol Cell, 2016. 62(2): p. 207-21.
} 


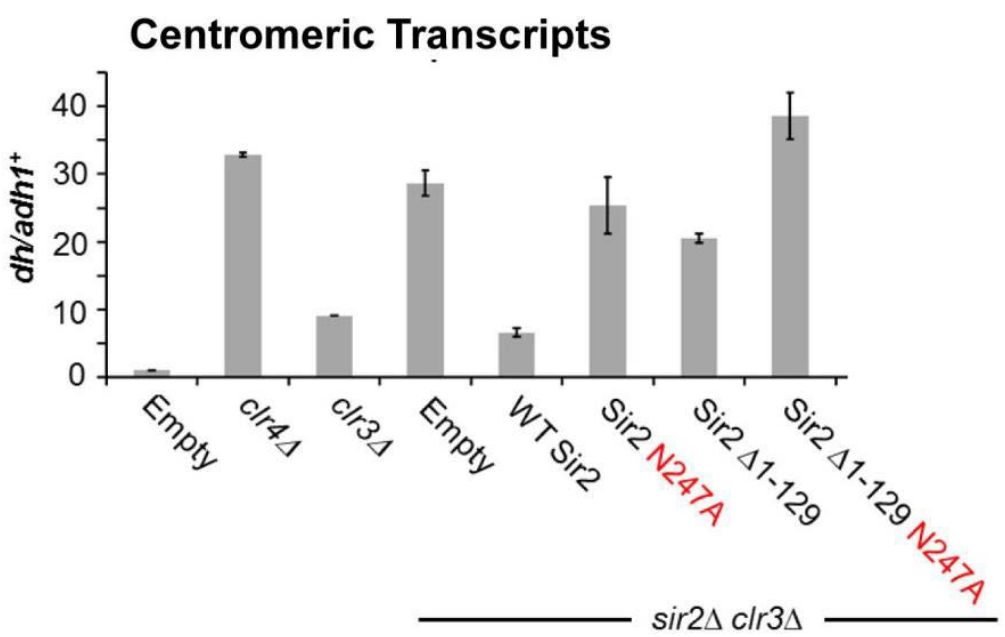

Figure 4-1. Sir2 $\Delta 1-129$ cannot maintain centromeric heterochromatin in the absence of Clr3.

Centromeric $d h$ transcripts normalized to the $a d h 1^{+}$control measured by qRT-PCR from a sir $2 \Delta \operatorname{clr} 3 \Delta$ double deletion strain transformed with the episomal Sir2 expression constructs. The high level of $d h$ transcripts in the $\operatorname{sir} 2 \Delta \operatorname{clr} 3 \Delta$ strain, which is similar to that of the $\operatorname{clr} 4 \Delta$ control, is reduced to $\operatorname{clr} 3 \Delta$ levels by transformation of episomal WT Sir2 but not Sir2 N247A or Sir2 $\Delta 1-129$. 
Figure 4-2. SHREC subunits show separation of function and overlap with Sir2 in transcriptional regulation.

A. UHC analysis of the top 1,000 most variable genes in strains deleted for individual SHREC components or Sir2 based on the median absolute deviation (MAD) score. Blue lines represent lower transcript expression while red lines represent highly expressed transcripts. Overlaps in transcriptional regulation were observed between $\operatorname{clr} 1 \Delta, \operatorname{clr} 2 \Delta$, and $\operatorname{clr} 3 \Delta$ strains while $\operatorname{sir} 2 \Delta, \operatorname{chp} 2 \Delta$, and mit $1 \Delta$ strains share a high degrees of overlap. B. Venn diagram showing overlap in differentially regulated genes with differences greater than 1.5-fold. Colors correspond to the number of overlaps between genotypes (blue- 2 genotypes, red-3 genotypes, white-4 genotypes, and black-5 genotypes). C. Graph showing RNA-seq data for the number of repetitive centromeric sequences in SHREC deletion backgrounds normalized to WT. All SHREC components show upregulation of centromeric transcripts, with $\operatorname{clr} 1 \Delta, \operatorname{clr} 2 \Delta$, and $\operatorname{clr} 3 \Delta$ strains having the highest centromeric transcript levels. D. qRT-PCR validation to confirm the RNA-seq results showing transcriptional overlap between $\operatorname{clr} 1 \Delta, \operatorname{clr} 2 \Delta$, and $\operatorname{clr} 3 \Delta$ strains and $\operatorname{sir} 2 \Delta$, $\operatorname{chp} 2 \Delta$, and mit $1 \Delta$ strains.

Source: Modified with permission from Elsevier Inc. Job, G., C. Brugger, T. Xu, B.R. Lowe, Y. Pfister, C. Qu, S. Shanker, J.I. Banos Sanz, J.F. Partridge, and T. Schalch, SHREC Silences Heterochromatin via Distinct Remodeling and Deacetylation Modules. Mol Cell, 2016. 62(2): p. 207-21. Creative Commons license: https://creativecommons.org/licenses/by/4.0/. DOI: 10.1016/j.molcel.2016.03.016. 
A.

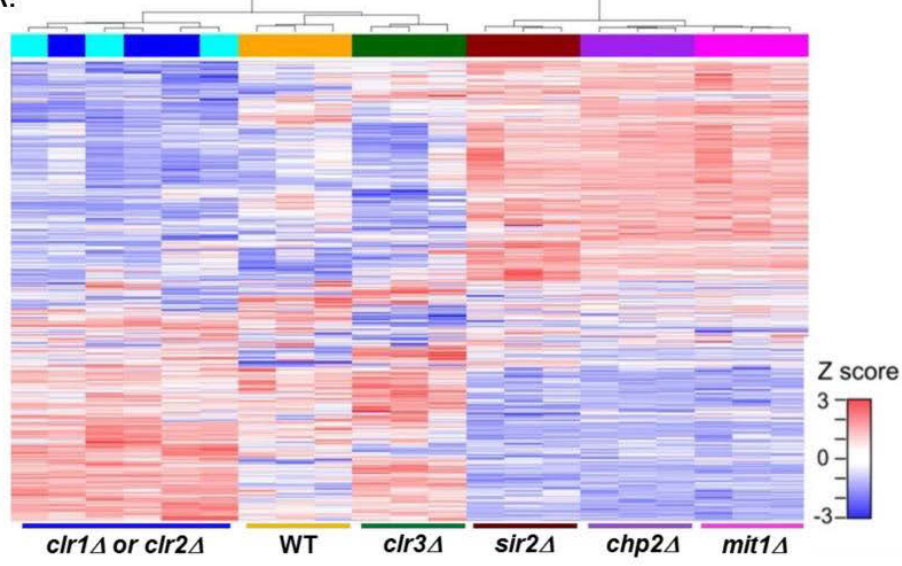

B.

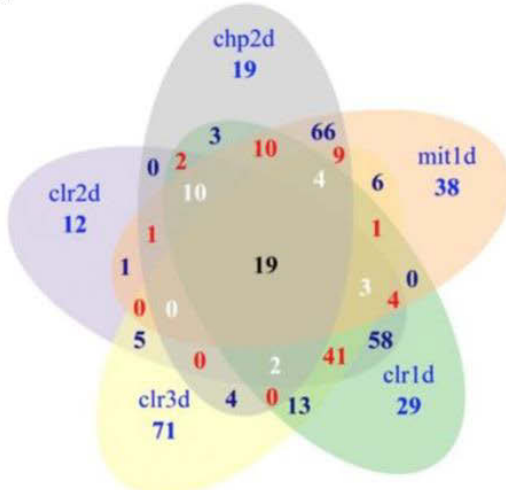

C.

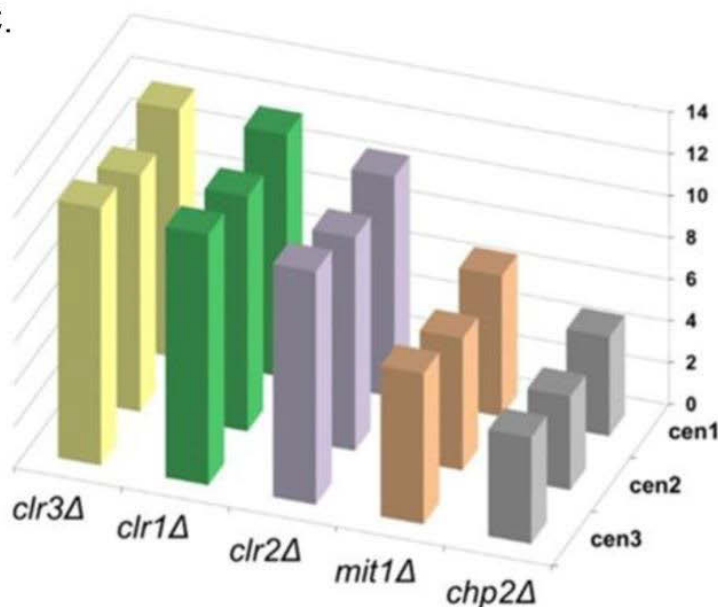

D. $\quad$ SPBC1289.14.1 Transcripts

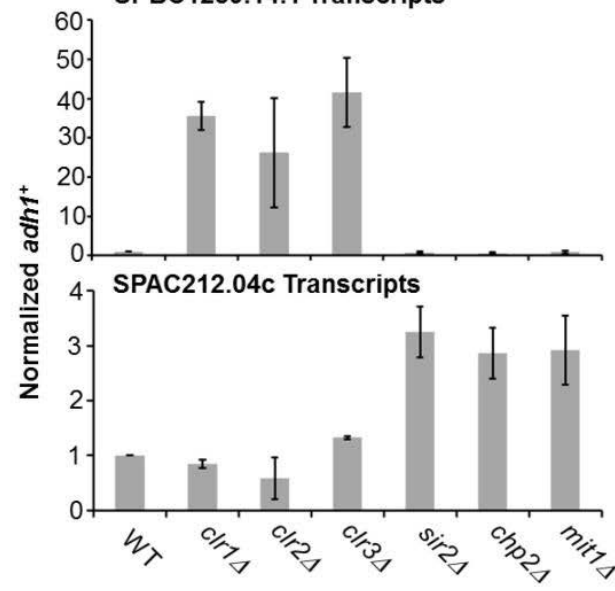


score indicated a distinction in transcriptional regulation between different members of the SHREC complex (Figure 4-2A). Although deletion of each SHREC component upregulated expression of constitutive heterochromatic regions as expected, overall gene expression patterns could be grouped into two categories with only 19 genes, which are mainly heterochromatic, showing overlap between all components (Figure 4-2B). The analysis indicated that $\operatorname{clr} 1 \Delta, \operatorname{clr} 2 \Delta$, and $\operatorname{clr} 3 \Delta$ showed significant overlap in expression profiles, agreeing with previous profiling indicating similar gene expression regulation between $\operatorname{clr} 1 \Delta$ and $\operatorname{clr} 3 \Delta$ [205]. Within this group, $\operatorname{clr} 1 \Delta$ and $\operatorname{clr} 2 \Delta$ showed the most overlap while clr3 $\Delta$ showed more changes in comparison. Together, there were 65 genes which showed an overlap in regulation between $\operatorname{clr} 1 \Delta, \operatorname{clr} 2 \Delta$, and $\operatorname{clr} 3 \Delta$. Conversely, both $\operatorname{chp} 2 \Delta$ and mit $1 \Delta$ shared significant overlap between their expression profiles which differed more significantly from the WT control, showing 119 genes with overlapping regulation. Specifically, at the centromere $\operatorname{clr} 1 \Delta, \operatorname{clr} 2 \Delta$, and $\operatorname{clr} 3 \Delta$ play a larger role in transcriptional regulation in comparison to mit $1 \Delta$ and $\operatorname{chp} 2 \Delta$, implying the separation of function also occurs at heterochromatic loci (Figure 4-2C). The changes observed in the RNA-seq profiling were validated on independent duplicate biological samples using qRT-PCR, confirming the validity of the data (Figure 4-2D). This data indicates there is a distinct pattern of regulation between different components of SHREC, with $\operatorname{clr} 1 \Delta$, $\operatorname{clr} 2 \Delta$, and $\operatorname{clr} 3 \Delta$ clustering together and $\operatorname{chp} 2 \Delta$ and mit $1 \Delta$ clustering together. Although there is a minimal level of overlap between all SHREC components, this data suggests that SHREC may act in separate entities to regulate gene expression.

\section{Sir2 Shows Overlap with the Mit1/Chp2 Sub-Complex}

Since Sir2 and Clr3 act redundantly in centromeric heterochromatin maintenance, we also wanted to examine potential overlaps between SHREC component deletions and $\operatorname{sir} 2 \Delta$ strains in global transcriptional analysis by RNA-seq. Currently, little is known about the role of Sir2 within the cell beyond its involvement in heterochromatic silencing and no global transcriptional analysis of $\operatorname{sir} 2 \Delta$ cells has previously been undertaken. Intriguingly, sir $2 \Delta$ exhibits very little transcriptional overlap with $\operatorname{clr} 1 \Delta, \operatorname{clr} 2 \Delta$, and $\operatorname{clr} 3 \Delta$ in the UHC analysis of RNA-seq data (Figure 4-2A). In contrast, sir $2 \Delta$ exhibits a high degree of overlap with mit $1 \Delta$ and $\operatorname{chp} 2 \Delta$. The overlap in regulation between sir $2 \Delta$, mit $1 \Delta$, and $\operatorname{chp} 2 \Delta$ was validated by qRT-PCR as previously described (Figure 4-2D). This data which, suggests that Sir2 may act in the same pathway as Mit1 and Chp2, provides insight into how Sir2 and Clr3 may function redundantly in transcriptional control of certain loci.

We further tested the idea that Sir2 may be acting in the same pathway as Mit1 and Chp2. To do this, we combined a sir2 $\Delta$ with deletions of each SHREC component individually. If Sir2 is acting in the same pathway as Mit1 and Chp2, it would be expected that no change in transcription would be seen at loci with overlapping regulation identified in the RNA-seq analysis when sir $2 \Delta$ is combined with either mit $1 \Delta$ or $\operatorname{chp} 2 \Delta$ to produce a double mutant strain. In contrast, combining $\operatorname{sir} 2 \Delta$ with $\operatorname{clr} 1 \Delta$, $\operatorname{clr} 2 \Delta$, or $\operatorname{clr} 3 \Delta$ should alter transcriptional regulation due to affecting both SHREC sub- 
complexes. We tested our combined deletions by qRT-PCR at several loci that were differentially regulated by the sub-complexes (Figure 4-3). In preliminary experiments, we found combining $\operatorname{sir} 2 \Delta$ with a $\operatorname{clr} 1 \Delta, \operatorname{clr} 2 \Delta$, or $\operatorname{clr} 3 \Delta$ did produce an alteration in transcription, while combining $\operatorname{sir} 2 \Delta$ with either mit $1 \Delta$ or $\operatorname{chp} 2 \Delta$ did not dramatically alter transcription. This indicates that there is likely some overlap in the pathway that Mit1, Chp2, and Sir2 are acting in, but further validation will be required to confirm this idea.

\section{CIr3 Can Deacetylate Residues in the H2B N-Terminal Tail}

The known histone target of Clr3 is deacetylation of H3K14. Since no comprehensive studies examining additional $\mathrm{Clr} 3$ targets have been undertaken, it is possible that Clr3 has targets beyond H3K14 deacetylation. Recent evidence from $N$. crassa has shown the Clr3 homolog HDA1 can deacetylate $\mathrm{H} 2 \mathrm{~B}$ on several N-terminal lysine residues which are conserved in fission yeast (Figure 4-4A) [151]. Although studies have been done in vitro and in vivo examining histone $\mathrm{H} 3$ and $\mathrm{H} 4$ deacetylation by $\mathrm{Clr} 3$, no studies have looked at the role of $\mathrm{Clr} 3$ in $\mathrm{H} 2 \mathrm{~B}$ deacetylation. Here we examined the possibility that $\mathrm{H} 2 \mathrm{~B}$ is deacetylated by $\mathrm{Clr} 3$ and whether this might contribute to the role of $\mathrm{Clr} 3$ in heterochromatic silencing.

The hypothesis that the N-terminus of $\mathrm{H} 2 \mathrm{~B}$ is deacetylated by $\mathrm{Clr} 3$ was supported by the observation of decreased $\mathrm{H} 2 \mathrm{~B}$ signal when western blotting was performed against $\mathrm{H} 2 \mathrm{~B}$ using extract from $c l r 3 \Delta$ cells (Figure 4-4B). The Bioacademia anti-H2B antibody used in the experiment was raised against an N-terminal fission yeast $\mathrm{H} 2 \mathrm{~B}$ peptide (SAAEKKPASKAPAGK) which contains the conserved lysine residues we hypothesized might be deacetylated by $\mathrm{Clr} 3$. To confirm this decreased signal is unique to $\mathrm{H} 2 \mathrm{~B}$ in a clr3 4 , we blotted for $\mathrm{H} 2 \mathrm{~A}, \mathrm{H} 3$, and $\mathrm{H} 4$ also. No decrease in $\mathrm{H} 2 \mathrm{~A}, \mathrm{H} 3$, or $\mathrm{H} 4$ was observed. We also tested whole cell extract (WCE) from $\operatorname{clr} 1 \Delta, \operatorname{clr} 2 \Delta$, and $\operatorname{sir} 2 \Delta$ and found no decrease in the $\mathrm{H} 2 \mathrm{~B}$ signal in these backgrounds, indicating that the decrease in H2B signal was unique to $\operatorname{clr} 3 \Delta$ cells. Since the decrease in signal is unique to $\operatorname{clr} 3 \Delta$ cells, it was possible that acetylation of lysines within the NTD of H2B blocked antibody recognition. This provided evidence that $\mathrm{Clr} 3$ may deacetylate the NTD of $\mathrm{H} 2 \mathrm{~B}$ at the conserved lysine residues.

In order to confirm that $\mathrm{H} 2 \mathrm{~B} \mathrm{K5}, 6,10$, and 15 are deacetylated by $\mathrm{Clr} 3, \mathrm{MS} / \mathrm{MS}$ was utilized to identify increases in histone acetylation in our $\operatorname{clr} 3 \Delta$ strain. Histones were isolated from WT and clr3 $\Delta$ cells by acid extraction and cation exchange before being subjected to two rounds of HPLC purification (Figure 4-5A). This allowed for the purification of histones suitable for LC-MS/MS analysis to detect PTMs. The fractions obtained after HPLC were subjected to both immunoblotting and coomassie staining to analyze purity (Figure 4-5B). The purified fractions containing histones were sent to Alan Tackett (UAMS) who performed differential in vitro chemical derivatization with $d_{0}$ or $d_{10}$ propionic anhydride. This adds 5Da to peptides from one sample to differentiate between peptides from WT and clr3 $\Delta$ samples, allowing us to perform a quantitative analysis of different peptides to determine changes in PTMs [200]. The results of the 


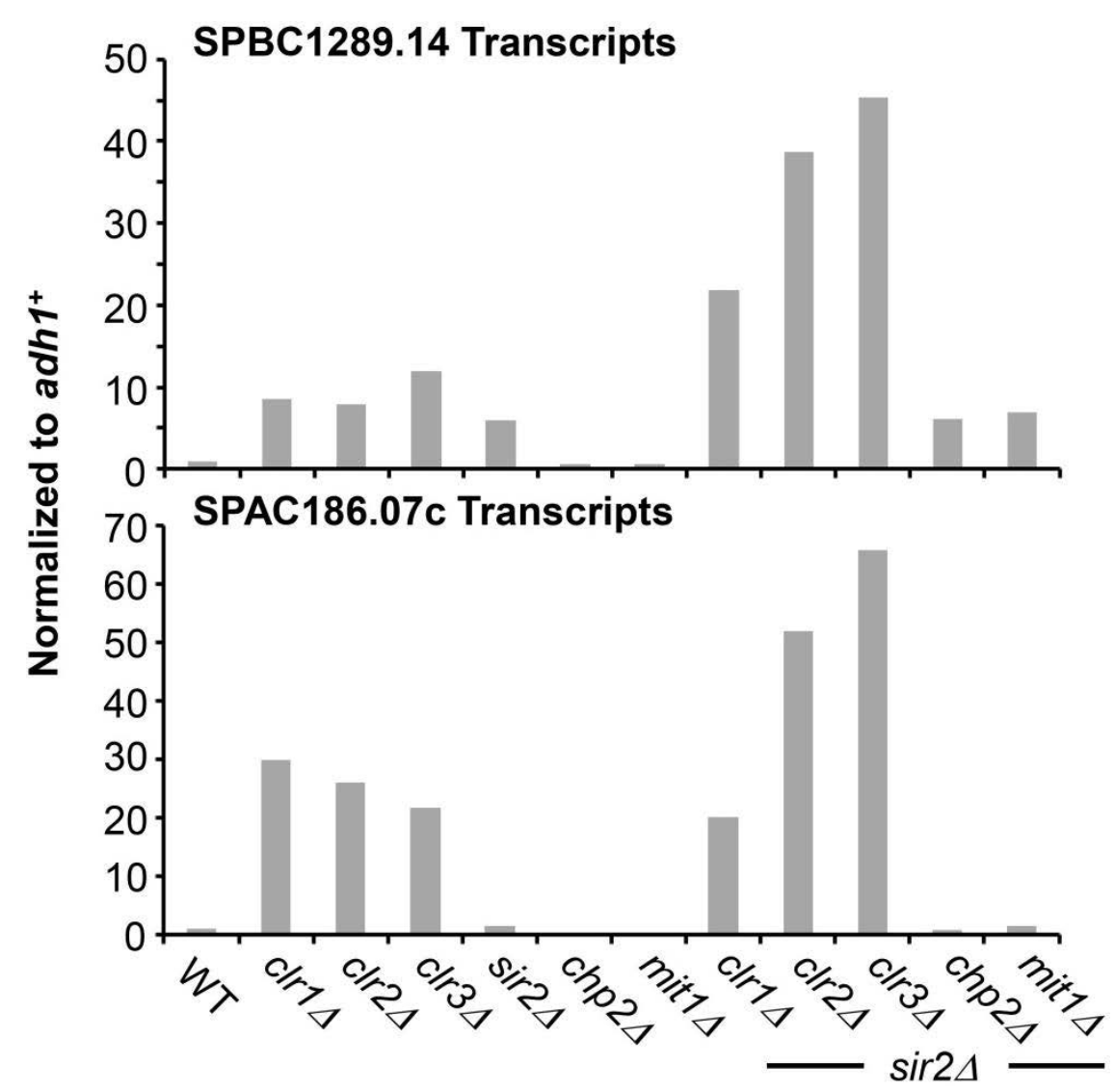

Figure 4-3. Sir2 deletion strains show overlaps in transcriptional regulation with both $\operatorname{chp} 2 \Delta$ and mit1 $\Delta$ strains.

qRT-PCR of specific genes showing differential regulation in RNA-seq. Deletion of Clr1, $\mathrm{Clr} 2$, and $\mathrm{Clr} 3$ in strains bearing $\operatorname{sir} 2 \Delta$ show additive defects at these differentially regulated loci, while deletion of Mit 1 or Chp1 in strains bearing sir $2 \Delta$ showed minimal additive defects in transcriptional regulation. 
A.

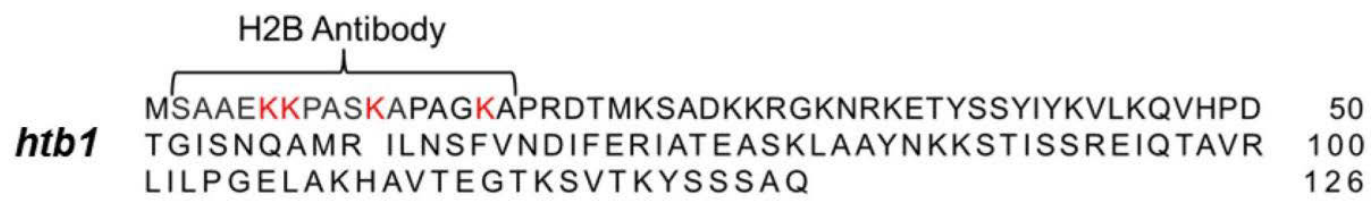

B.

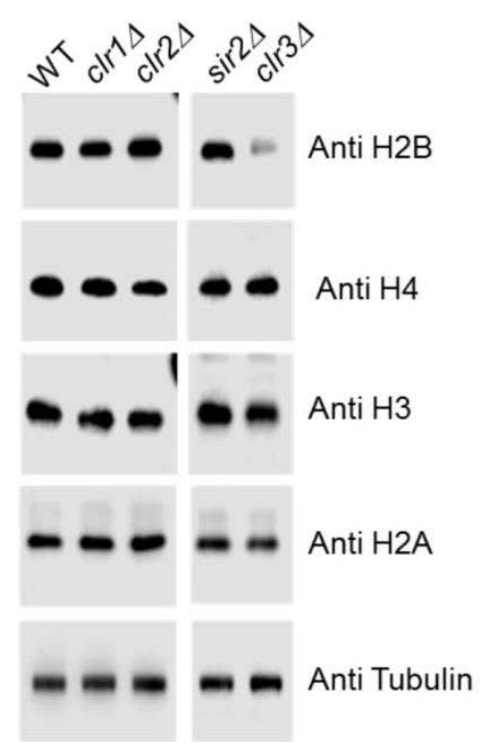

Figure 4-4. Deletion of Clr3 alters recognition of H2B by a H2B NTD-specific antibody.

A. Sequence of fission yeast $h t b 1^{+}$gene which encodes histone H2B. The Bioacademia $\mathrm{H} 2 \mathrm{~B}$ antibody was generated against the indicated $\mathrm{N}$-terminal peptide which contains several conserved lysines shown in red. B. Immunoblotting of WCE from WT, clr $1 \Delta$, $\operatorname{clr} 2 \Delta$, sir $2 \Delta$, and $\operatorname{clr} 3 \Delta$ strains. H2B recognition is specifically decreased in WCE from $\operatorname{clr} 3 \Delta$ cells while levels of other histones remain consistent. 
A.

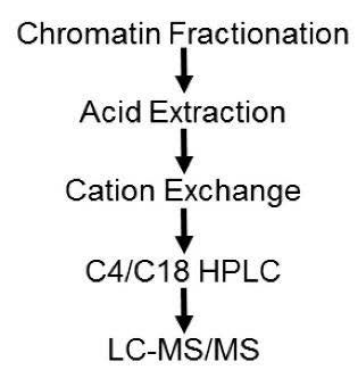

C.

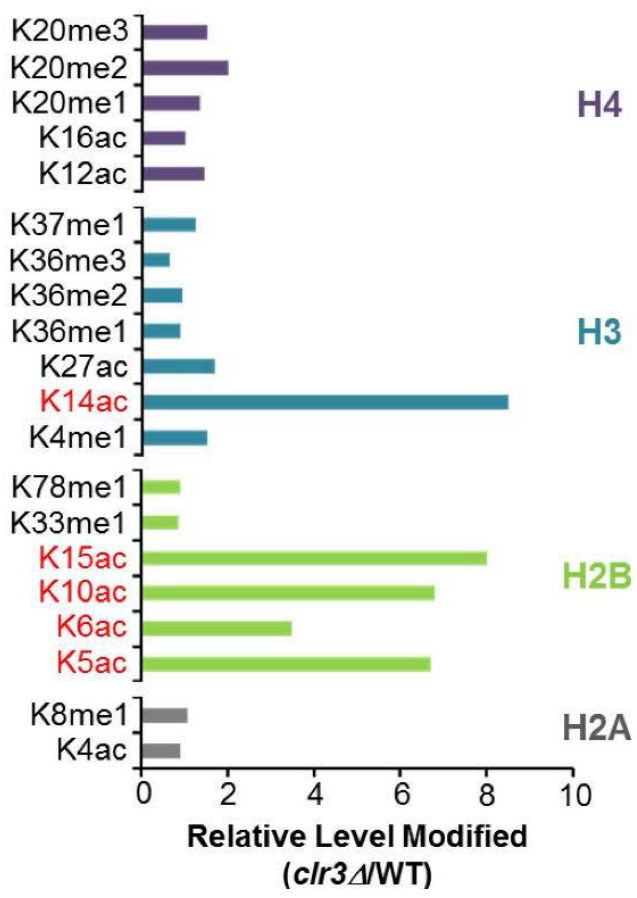

B.
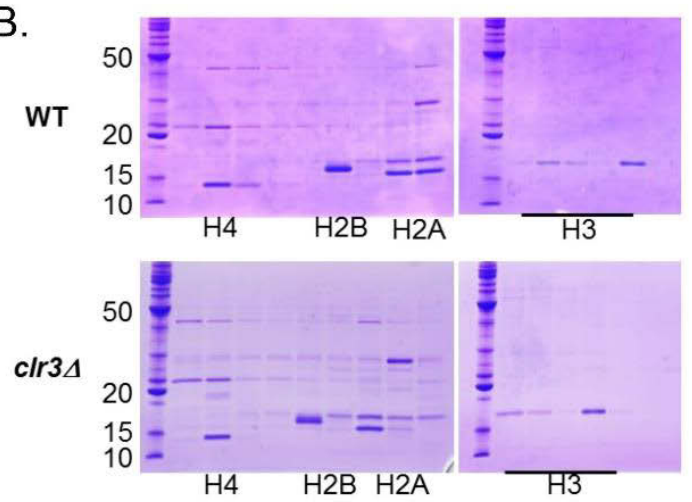

D.

4

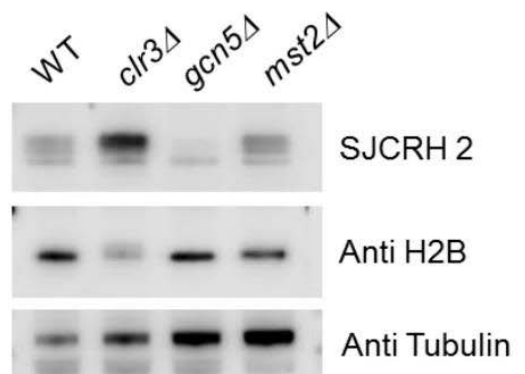

Figure 4-5. Clr3 deacetylates $\mathrm{H2B}$ K5, 6, 10, and 15 in fission yeast.

A. Diagram of the fission yeast histone purification scheme. We first isolated a chromatin fraction which is subjected to acid extraction, cation exchange, and two round of HPLC to obtain fractions pure enough for MS/MS analysis. B. Coomassie gel of C18 HPLC eluted fractions of histones purified from WT and $\operatorname{clr} 3 \Delta$ strains. Highly pure bands can be seen for H2A, H2B, H3, and H4. C. LC-MS/MS analysis showing relative levels of histone PTMs in clr3 $\Delta$ strain compared to WT. In the $c l r 3 \Delta$ strain, 3-8 fold increases in acetylation of H2B K5, 6, 10, and 15 were observed, along with an increase in H3K14ac. D. Immunoblotting using an antibody against H2B K5,6,10,15ac (SJCRH2) shows increased signal in $\operatorname{clr} 3 \Delta \mathrm{WCE}$ and decreased signal in gcn5 $\Delta \mathrm{WCE}$ compared to WT WCE, suggesting Gcn5 can acetylate these residues.

Source: MS/MS shown in panel C was performed by Alan Tackett on histones purified in our lab [unpublished communication on 05/20/2014]. 
MS/MS showed that H3K14ac was increased 8-fold in $\operatorname{clr} 3 \Delta$ as expected (Figure 4-5C) [unpublished communication from Alan Tackett on 05/20/2014]. Also, increased acetylation of 3 to 8-fold over WT was observed on H2B K5, 6, 10, and 15 in the clr3A strain. No other changes in PTMs were observed when all histones were examined. These results indicate that $\mathrm{H} 2 \mathrm{~B} \mathrm{K5}, 6,10$, and 15 are targeted by $\mathrm{Clr} 3$ for deacetylation.

To further verify our findings, we developed an antibody against an $\mathrm{H} 2 \mathrm{~B} \mathrm{~N}-$ terminal peptide with acetylation on $\mathrm{K} 5,6,10$, and 15 to monitor acetylation using immunoblotting. The antibodies were produced by Calico Biologicals Inc. in Stevens PA using rabbits injected with the acetylated $\mathrm{H} 2 \mathrm{~B} \mathrm{~N}$-terminal peptide. A total of four serums were tested and the best serum, SJCRH2, was used for further experiments. The antibodies were tested on WCEs of both WT and $\operatorname{clr} 3 \Delta$ fission yeast to show these marks can be detected in vivo through immunoblotting (Figure 4-5D). While loss of recognition of $\mathrm{H} 2 \mathrm{~B}$ by the Bioacademia $\mathrm{H} 2 \mathrm{~B}$ antibody was observed in $c l r 3 \Delta$ extract, higher signal was observed using the SJCRH2 antibody which detects H2B K5, 6, 10, and 15ac. This data further confirms that $\mathrm{H} 2 \mathrm{~B} \mathrm{K5}, 6,10$, and 15 are acetylated in the absence of Clr3, while only minor acetylation of these residues is observed in the WT strain.

We then utilized the H2B NTD K5, 6, 10, and 15ac antibody, SJCRH2, to identify whether the HATs Gcn5 or Mst 2 can acetylate these residues on H2B (Figure 4-5D). Whole cell extracts from gcn5 $\Delta$ and $m s t 2 \Delta$ were analyzed using immunoblotting. Previous work has shown Gcn5 can acetylate $\mathrm{H} 2 \mathrm{~B}$, and together with Mst2 is responsible for H3K14ac [206, 207]. Immunoblotting showed decreased levels of acetylated H2B in gcn $5 \Delta$ cells compared to WT by immunoblotting using the SJCRH2 antibody. In contrast, no significant decrease in $\mathrm{H} 2 \mathrm{~B} \mathrm{~K} 5,6,10$, and $15 \mathrm{ac}$ was observed in $m s t 2 \Delta$ cells using the SJCRH2 antibody. These preliminary results indicate that Gen5 appears to be the main HAT responsible for acetylating these sites.

\section{Mutation of H2B K5, 6, 10, and 15 Does Not Impact Heterochromatin Formation}

To test the impact of $\mathrm{H} 2 \mathrm{~B}$ acetylation at $\mathrm{K} 5,6,10$, and 15 we mutated the genomic $\mathrm{H} 2 \mathrm{~B}$ locus. Fission yeast have only a single gene coding for $\mathrm{H} 2 \mathrm{~B}, h t b 1^{+}$. In order to generate these mutations, we utilized a stain containing a genomic htbl-Flag allele from Jason Tanny (McGill University) in order to easily track H2B [208]. To knock in the mutations, a diploid fission yeast strain was created having both WT H2B and H2B-Flag. H2B-Flag was then knocked out with the $u r a 4^{+}$reporter gene, which was then replaced with either $\mathrm{H} 2 \mathrm{~B}$ K5, 6, 10, 15R (H2B 4R) to mimic deacetylation of the residues or $\mathrm{H} 2 \mathrm{~B} \mathrm{K5}, 6,10,15 \mathrm{Q}$ (H2B 4Q) to mimic acetylation. Strains were converted back to a haploid state with the sole copy of H2B being either H2B-Flag 4Q or 4R. Both the H2B 4Q and 4R strains were viable. The loss of WT H2B was confirmed by western blotting with the bioacademia $\mathrm{H} 2 \mathrm{~B}$ antibody which could not recognize the H2B 4Q or $4 \mathrm{R}$ variants (Figure 4-6A). We also noted that $\mathrm{H} 2 \mathrm{~B} 4 \mathrm{R}$ runs at a lower molecular weight than WT H2B while H2B 4Q runs at a slightly higher molecular weight. This allowed the confirmation of the presence of $\mathrm{H} 2 \mathrm{~B} 4 \mathrm{Q}$ or $4 \mathrm{R}$ as the only copy of $\mathrm{H} 2 \mathrm{~B}$ present in the 
A.
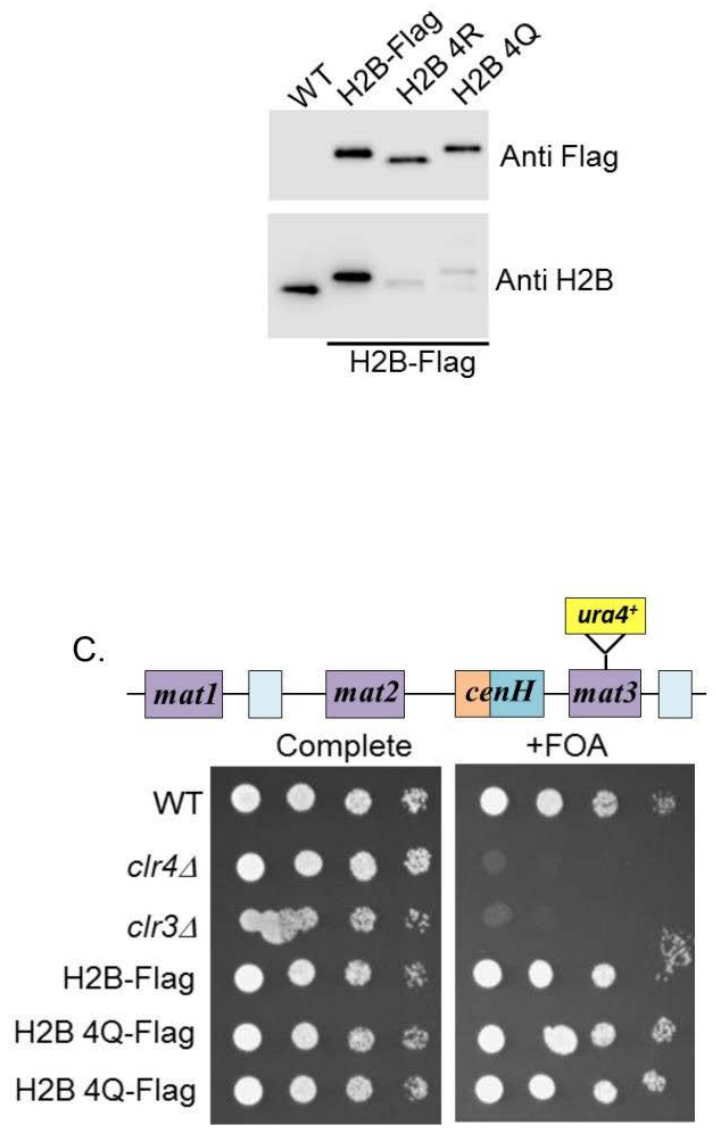

B.
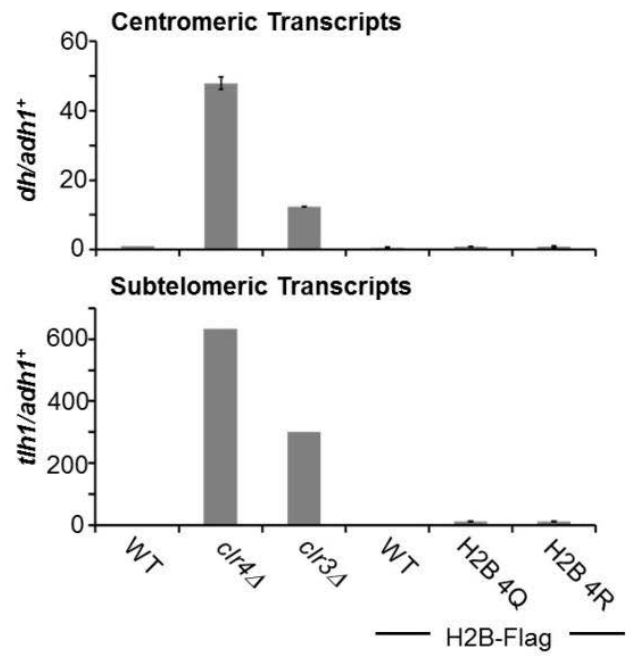

D.

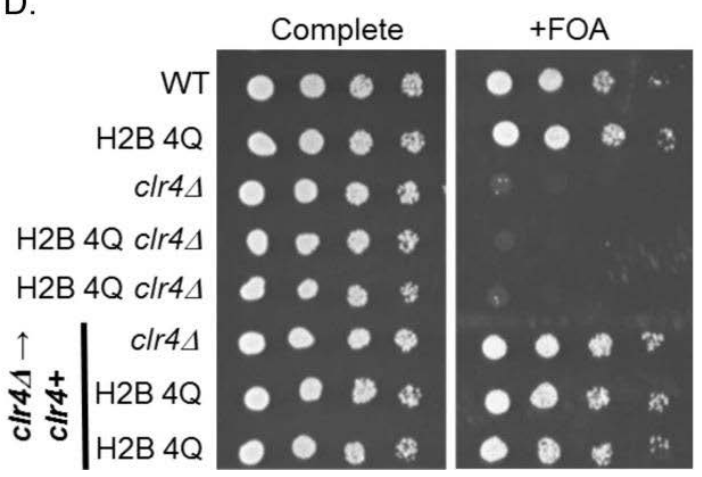

Figure 4-6. The H2B K5, 6, 10, and 15 acetyl mimetic does not impact the maintenance or establishment of heterochromatin.

A. Immunoblotting of $\mathrm{H} 2 \mathrm{~B}$ in WCE from H2B 4Q and 4R mutant strains. The Bioacademia antibody shows little recognition of $\mathrm{H} 2 \mathrm{~B} 4 \mathrm{Q}$ or $4 \mathrm{R}$, while anti-Flag can detect $\mathrm{H} 2 \mathrm{~B}$ in all strains where $\mathrm{H} 2 \mathrm{~B}$-Flag is present, indicating the expression of the $\mathrm{H} 2 \mathrm{~B}$ mutants. B. Centromeric $d h$ and telomeric $t h l$ transcripts normalized to the $a d h 1^{+}$ euchromatic control measured by qRT-PCR from H2B 4Q and 4R strains. H2B 4Q and 4R show no increase in centromeric or telomeric transcripts in comparison to $\operatorname{clr} 3 \Delta$ cells and $\operatorname{clr} 4 \Delta$ cells. C. Silencing assay monitoring silencing at mat using a mat3-M::ura4 ${ }^{+}$ reporter gene monitoring growth on 5-FOA containing medium. H2B 4Q cells show no defect in mat silencing in contrast to clr3 $\Delta$ cells, which are defective for silencing at this locus. D. Centromeric establishment assay for $\mathrm{H} 2 \mathrm{~B} 4 \mathrm{Q}$ cells monitoring expression of an otr $1 R$ :: ura $4^{+}$reporter gene. $\operatorname{clr} 4^{+}$was reintegrated into the H2B 4Q clr4 $\Delta$ strain, sole copy into the endogenous $\operatorname{clr} 4^{+}$locus. The H2B 4Q $\operatorname{clr} 4^{+}$establishment strain can properly establish centromeric heterochromatin at levels similar to the WT $\operatorname{clr} 4^{+}$ reintroduction stain. 
strains, allowing these strains to be utilized for further experiments examining the effects of $\mathrm{H} 2 \mathrm{~B}$ acetylation on heterochromatin formation.

Since $\operatorname{clr} 3 \Delta$ shows a small defect in heterochromatin maintenance, we tested the maintenance of heterochromatin in the H2B 4Q or 4R mutants using qRT-PCR to examine both centromeric $d h$ transcripts and subtelomeric thl transcripts in the H2B mutant backgrounds (Figure 4-6B). At the centromere, while $d h$ transcripts show a slight increase above WT in clr3 $\Delta$ cells, our H2B 4Q and 4R mutants exhibited no increase in pericentromeric transcripts. This indicates acetylation of $\mathrm{H} 2 \mathrm{~B} \mathrm{K5}, 6,10$, and 15 does not affect centromeric heterochromatin maintenance. At the telomere, $t h l$ transcripts were similar to WT levels in comparison to the $\operatorname{clr} 3 \Delta$ strain, which has high levels of subtelomeric transcripts. This indicates the maintenance of telomeric heterochromatin is not affected by acetylation at $\mathrm{H} 2 \mathrm{~B} \mathrm{~K} 5,6,10$, and 15 . We also examined the maintenance of heterochromatin at mat, using a silencing assay with a $u r a 4^{+}$reporter inserted at this locus (Figure 4-6C). While clr3 $\Delta$ cells exhibit a strong defect in the maintenance of heterochromatin, acetylation of $\mathrm{H} 2 \mathrm{~B} \mathrm{K5}, 6,10$, and 15 has no impact on heterochromatin maintenance at mat, and appear similar to WT. Together, these results suggest that deacetylation of $\mathrm{K} 5,6,10$, and 15 plays no role in heterochromatin maintenance at the major heterochromatic loci in fission yeast.

Although acetylation of K5, 6, 10, and 15 did not impact heterochromatin maintenance, we also tested if there is an impact on heterochromatin establishment since clr3 $\Delta$ impacts establishment. To do this, the H2B 4R and 4Q strains were crossed with a $\operatorname{clr} 4 \Delta$ and $\operatorname{clr} 4^{+}$was then reintroduced into the genomic locus sole copy. The ability of heterochromatin to re-establish in these strains was monitored by a centromeric silencing assay (Figure 4-6D). The $\operatorname{clr} 4 \Delta$ to $\mathrm{clr} 4^{+}$control strain properly re-established centromeric heterochromatin as expected. Similar to the WT strain, both H2B-4Q and 4R strains were also able to re-establish centromeric heterochromatin. This indicates that acetylation at $\mathrm{H} 2 \mathrm{~B} \mathrm{K5}, 6,10$, and 15 does not impact the de novo establishment of centromeric heterochromatin.

\section{H2B 4Q Has Minimal Global Transcriptional Changes}

Since there were no heterochromatic defects associated with acetylation mimetics of $\mathrm{H} 2 \mathrm{~B} \mathrm{K5}, 6,10$, and 15, we next examined global transcriptional regulation in the $\mathrm{H} 2 \mathrm{~B}$ 4Q mutant to examine the potential impact of acetylation of these residues. To do this, RNA-seq profiling was utilized to look at overall transcriptional changes of H2B 4Q strain in comparison to $\operatorname{clr} 1 \Delta, \operatorname{clr} 2 \Delta$, and $\operatorname{clr} 3 \Delta$ strains. Analysis of the RNA-seq data by UHC analysis examining the top 1,000 most variable genes indicated only minor changes in the H2B 4Q strain when compared to WT, with around 90 genes showing transcriptional changes which differ at least 1.5-fold from WT (Figure 4-7A). In contrast, $\operatorname{clr} 3 \Delta$ cells showed around $60 \%$ overlap with either $\operatorname{clr} 1 \Delta$ or $\operatorname{clr} 2 \Delta$ strains, with around 200 genes being affected in each mutant. Most of these changes were at pericentromeric and subtelomeric regions. Together, $\operatorname{clr} 1 \Delta, \operatorname{clr} 2 \Delta$, and $\operatorname{clr} 3 \Delta$ showed overlap in transcriptional regulation with $\mathrm{H} 2 \mathrm{~B} 4 \mathrm{Q}$ in 36 genes, while clr3 $\Delta$ cells and H2B 4Q cells 
A.

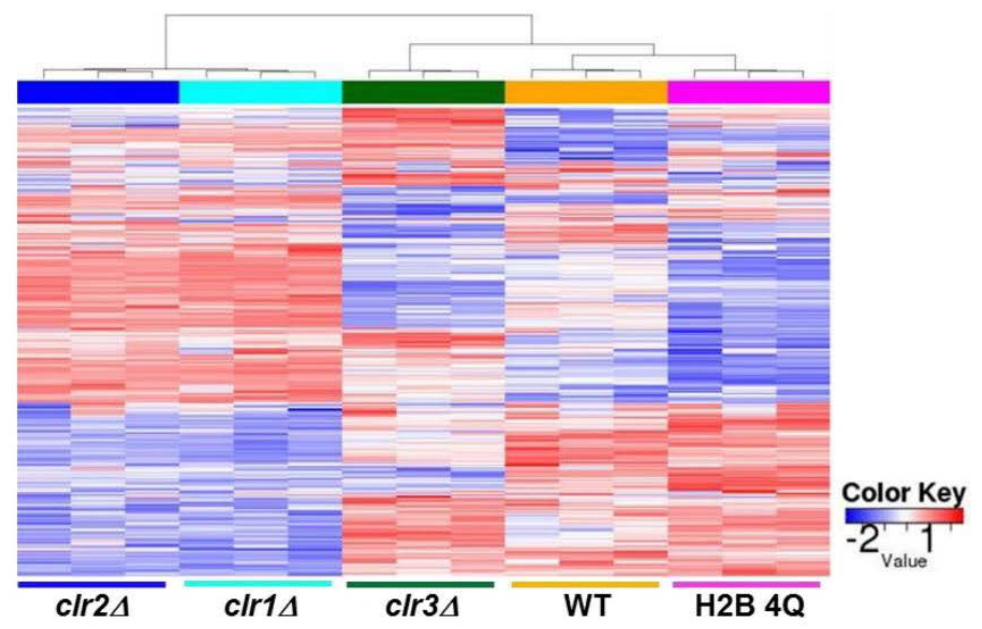

B.

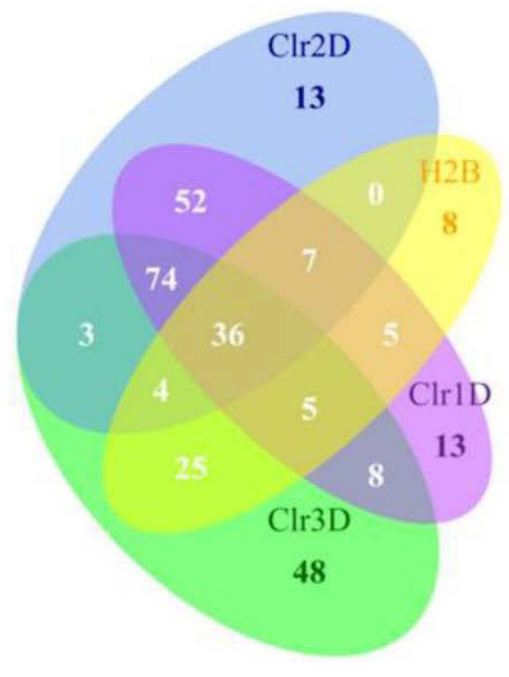

Figure 4-7. The H2B K5, 6, 10, and 15 acetyl mimetic has no major impact on transcriptional regulation.

A. UHC analysis of the top 1,000 most variable genes in $\operatorname{clr} 1 \Delta, \operatorname{clr} 2 \Delta, \operatorname{clr} 3 \Delta$ or $\mathrm{H} 2 \mathrm{~B} 4 \mathrm{Q}$ strains based on the MAD score. Blue lines represent lower transcript expression while red lines represent highly expressed transcripts. H2B 4Q showed only minimal global transcriptional changes in contrast to $\operatorname{clr} 1 \Delta$, $\operatorname{clr} 2 \Delta$, or $\operatorname{clr} 3 \Delta$ strains. B. Venn diagram showing overlap in differentially regulated genes having differences greater than 1.5 -fold. Only 90 genes showed transcriptional changes in H2B 4Q cells, 25 of which overlapped with $\operatorname{clr} 3 \Delta$ cells, with less overlap with $\operatorname{clr} 1 \Delta$ or $\operatorname{clr} 2 \Delta$ cells. 
showed the most overlap individually, with 25 of the 90 genes showing transcriptional changes in H2B 4Q cells showing changes in clr3 $\Delta$ cells (Figure 4-7B). Most of the overlap was at pericentromeric and subtelomeric regions, although the transcriptional changes were less severe in the H2B 4Q mutant strain. This raises the possibility that there could be a minor involvement of these sites of acetylation in regulation of heterochromatic silencing, possibly by altering the spreading or compaction of heterochromatin and nucleosome stabilization. 


\section{CHAPTER 5. INVESTIGATION OF DDX3X MUTATIONS FOUND IN PEDIATRIC MEDULLOBLASTOMA*}

\section{Human DDX3X Can Functionally Complement for Fission Yeast Ded1}

DDX3X has been implicated in many processes within the cell ranging from RNA metabolism to translation. The PCGP project identified eight somatic missense mutations of DDX3X associated with medulloblastoma [154]. Although DDX3X is frequently mutated, it is unclear how these mutations contribute to medulloblastoma. To examine defects in cellar processes caused by these mutations, we utilized the fission yeast model system. Unlike higher eukaryotes, fission yeast only has one DDX3X orthologue known as ded1. In fission yeast, dedl is an essential gene, so we utilized a thermosensitive mutant of Ded1, ded1-1D5 [162]. Our sequencing indicates the ded1-1D5 mutant has two missense mutations: S282F and R285C. While grown at the permissive temperature of $25^{\circ} \mathrm{C}$, the mutant Ded1 fully complements WT Ded1 function, but when grown at the non-permissive temperature of $36^{\circ} \mathrm{C}$, ded1-1D5 cannot complement WT Ded 1 and the cells die. This system serves as a tool which we utilized to examine the functional consequences of mutations in DDX3X using an episomally expressed version of DDX3X.

In order to test whether DDX3X can complement for Ded1 in the ded1-1D5 background, two vector constructs were created by cloning either WT DDX3X or Ded1 into a pREP-41 expression vector with a C-terminal 3x V5 tag. These constructs were then transformed into the ded1-1D5 background. A negative control was made by transforming the ded1-1D5 strain with an empty pREP-41 plasmid. At the permissive temperature of $25^{\circ} \mathrm{C}$, all the transformants grew normally (Figure 5-1A). At the nonpermissive temperature of $36^{\circ} \mathrm{C}$, both the episomally expressed Ded1 and DDX3X could rescue the temperature sensitive growth defect in contrast to the negative control, which showed a loss of growth. This indicates that episomally expressed DDX3X can complement for loss of Ded1 function. We also examined the levels of episomally expressed DDX3X and Ded1 in comparison to endogenous levels of Ded1 protein (Figure 5-1B). Immunoblotting indicated that the episomally expressed DDX3X-3x V5 is expressed at levels similar to the episomally expressed Ded1-3x V5. In turn, the episomally expressed Ded1-3x V5 is expressed at levels similar to endogenous Ded1 as detected using an antibody against endogenous fission yeast Ded1. This indicates that overexpression is not playing a role in the ability of DDX3X to complement the ded11D5 growth defect.

* Modified with permission from Elsevier Inc. Epling, L.B., C.R. Grace, B.R. Lowe, J.F. Partridge, and E.J. Enemark, Cancer-associated mutants of RNA helicase DDX $3 X$ are defective in RNA-stimulated ATP hydrolysis. J Mol Biol, 2015. 427(9): p. 1779-96. 
Figure 5-1. DDX3X functionally complements for fission yeast ded1-1D5 temperature sensitive growth defect.

A. Simple plating assay of fission yeast transformed with episomal DDX3X or Ded1. The temperature sensitive growth defect of $d e d 1-1 D 5$ at $36^{\circ} \mathrm{C}$ is rescued by episomal Ded 1 or DDX3X. B. Western blot monitoring expression levels of episomal DDX3X in comparison to endogenous Ded1. Episomal DDX3X-3x V5 is expressed at levels similar to episomal Ded1-3x V5 when using an anti-V5 antibody. Using an antibody against Ded1, episomal Ded1 is less than 2-fold overexpressed compared to endogenous Ded1, indicating DDX3X is expressed at levels similar to endogenous Ded1. C. Centromeric $d h$ transcripts normalized to the $a d h 1^{+}$euchromatic control were measured using qRT-PCR in the ded1-1D5 background. At the non-permissive temperature, ded1-1D5 shows no increase in centromeric transcripts in comparison to the $d c r 1 \Delta$ control. D. Western blot for Cig2-3x HA protein levels in ded1-1D5 cells expressing episomal Ded1 or DDX3X. The ded1-1D5 temperature sensitive defect correlates with reduced Cig2 protein levels at the non-permissive temperature of $36^{\circ} \mathrm{C}$. Complementation by episomal Ded1 or DDX3X can rescue the reduction in Cig2 protein levels.

Source: Reprinted with permission from Elsevier Inc. Epling, L.B., C.R. Grace, B.R. Lowe, J.F. Partridge, and E.J. Enemark, Cancer-associated mutants of RNA helicase $D D X 3 X$ are defective in RNA-stimulated ATP hydrolysis. J Mol Biol, 2015. 427(9): p. 1779-96. Creative Commons license: https://creativecommons.org/licenses/by/4.0/. DOI: 10.1016/j.jmb.2015.02.015. 
A.

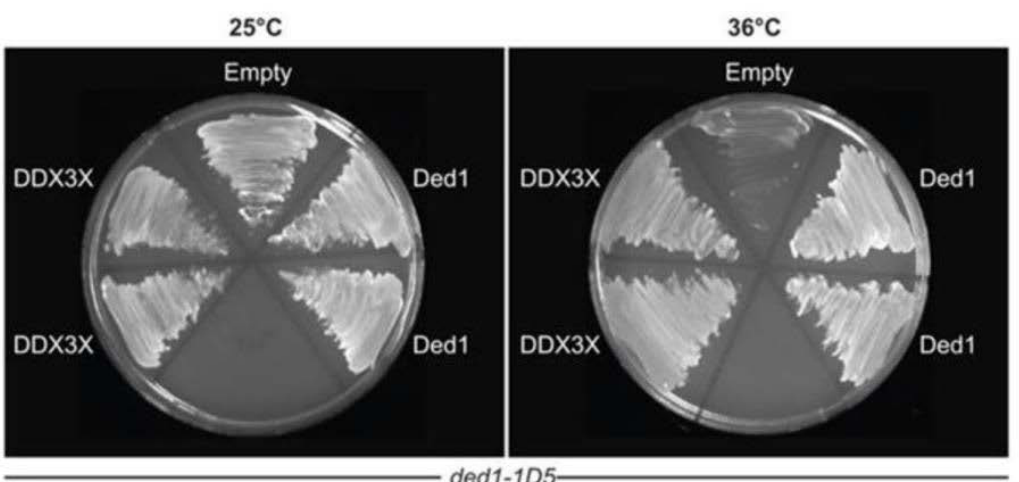

B.

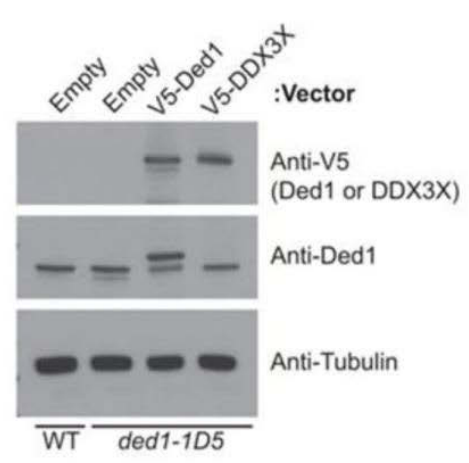

D.

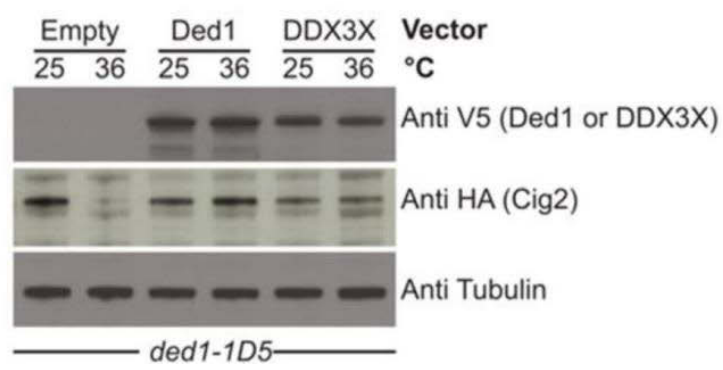

C.

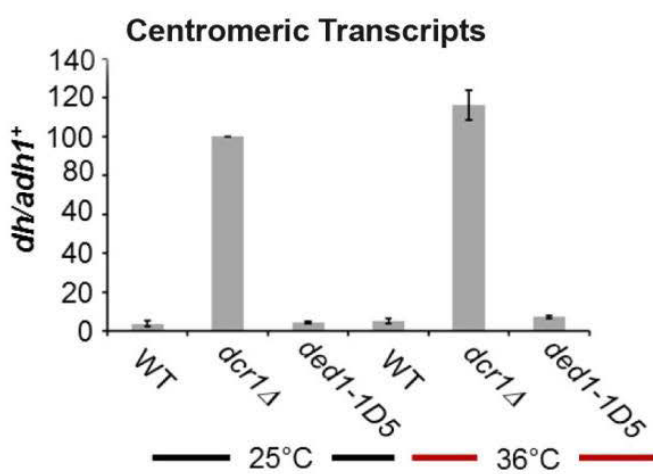




\section{Ded1 Is Not Involved in Silencing Pericentromeric Heterochromatin}

Since Ded1 was identified by mass spectrometry from an IP of fission yeast Stc1, a component which bridges RNAi to Clr-C, we tested whether it plays a role in heterochromatin formation [52]. To address this, a temperature shift assay was performed on ded1-1D5, dcr 1 1 , and WT. After growing overnight at $25^{\circ} \mathrm{C}$, the temperature was shifted to the non-permissive temperature of $36^{\circ} \mathrm{C}$ for 4 hours. After the incubation, $d h$ pericentromeric transcripts were measured using qRT-PCR and normalized to the euchromatic $a d h 1^{+}$control. At $25^{\circ} \mathrm{C}$, the $d c r \Delta$ strain showed high levels of $d h$ transcripts due to the disruption of the RNAi pathway (Figure 5-1C). In contrast, both WT and ded1-1D5 showed no transcript accumulation. At $36^{\circ} \mathrm{C}, d \operatorname{cr} 1 \Delta$ again showed high $d h$ transcript levels, while WT and ded1-1D5 strains showed no significant increase in transcripts. Based on this data, Ded1 does not appear to be involved in maintaining centromeric heterochromatin.

\section{Some DDX3X Medulloblastoma Mutants Are Defective for Complementation}

Since episomally expressed DDX3X can complement for ded1-1D5 growth defect, we then examined whether any of the DDX3X mutants associated with medulloblastoma were defective for complementation. C-terminally V5 tagged vectors of the nine cancer-associated DDX3X mutants (G302V, A222P, G325E, T275M, R351W, P568L, L353F, D354V, and M370R) were constructed. A negative control was made by mutating K230A of the Walker-A motif, which produces an ATPase-defective mutant. All mutants grew similarly to WT DDX $3 \mathrm{X}$ transformed cells at $25^{\circ} \mathrm{C}$ except the ATPasedefective K230A mutant which was defective for growth even at the permissive temperature in a simple plating assay (Figure 5-2A). When the cells were grown at the non-permissive temperature of $36^{\circ} \mathrm{C}, \mathrm{G} 302 \mathrm{~V}, \mathrm{~A} 222 \mathrm{P}, \mathrm{G} 325 \mathrm{E}, \mathrm{P} 568 \mathrm{~L}$, and K230A could not complement for the ded1-1D5 thermosensitive growth defect while T275M, R351W, L353F, D354V, and M370R showed complementation.

To further characterize the defects in complementation associated with the DDX3X mutations, we performed a 5-fold serial growth dilution assay at the semipermissive temperature of $33^{\circ} \mathrm{C}$ to measure growth of the defective DDX3X mutants (Figure 5-2B). Again, the K230A mutation showed the most severe growth defect, with decreased growth at both $25^{\circ} \mathrm{C}$ and $33^{\circ} \mathrm{C}$. The G302V, A222P, and G325E mutations showed significant growth defects at $33^{\circ} \mathrm{C}$ while the P568L mutation only showed a minor growth defect. The T275M, R351W, L353F, D354V, and M370R mutations showed no differences in growth at $33^{\circ} \mathrm{C}$. When examined by immunoblotting, DDX3X protein levels were shown to decrease when the cells were raised to the non-permissive temperature suggesting the possibility that the loss of function could be impacted by the decreased protein levels (Figure 5-3A).

We repeated the 5-fold serial dilution using a cold-sensitive mutant of ded 1 , ded 1 61 in order to address the issue with decreased protein levels [162]. In this background, at temperatures above $32^{\circ} \mathrm{C}$, ded1-61 is functional, but when the temperature is lowered to 
A.

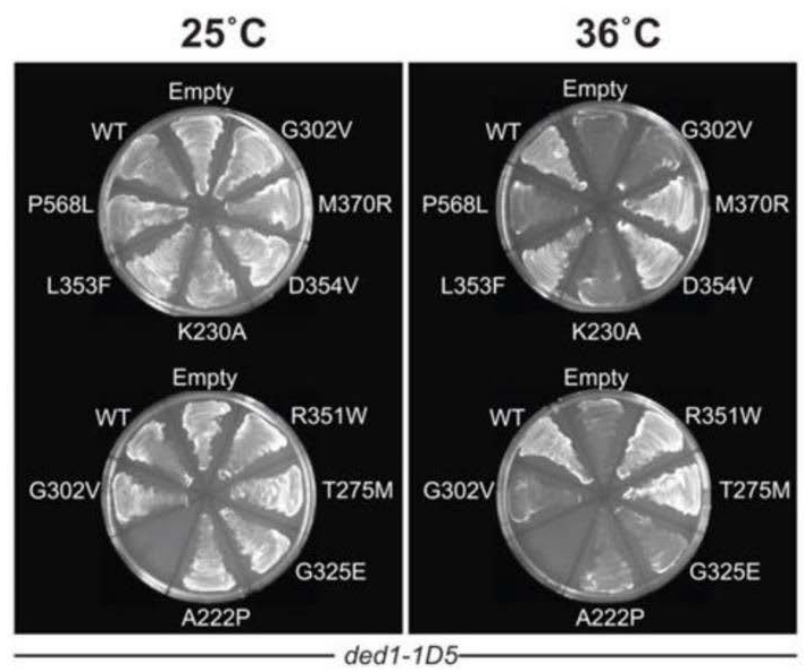

B.

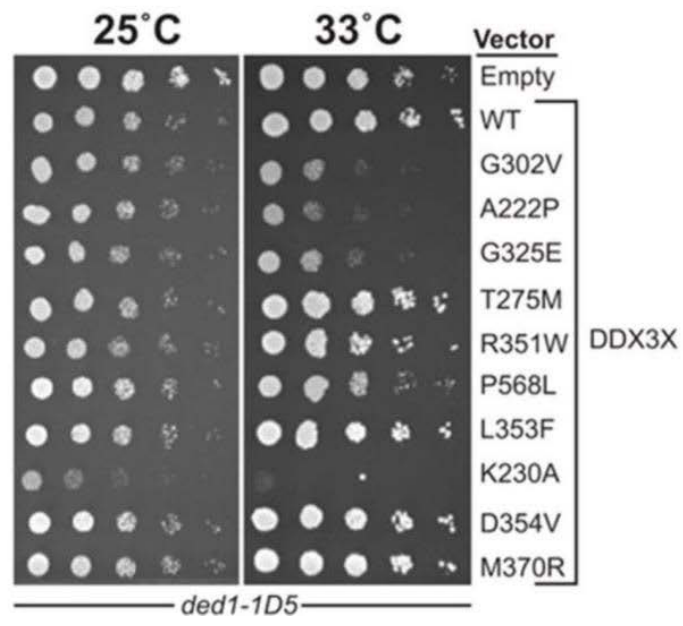

Figure 5-2. Specific DDX3X mutants cannot complement for loss of ded1-1D5 function.

A. Simple plating assay testing the complementation of Ded1 by episomal DDX3X cancer associated mutants transformed into the ded1-1D5 strain. The A222P, G302V, G325E, and P568L DDX3X mutants cannot complement for the ded1-1D5 temperature sensitive defect at $36^{\circ} \mathrm{C}$. B. Five-fold serial dilution of episomal DDX3X mutants transformed into the ded1-1D5 strain. A222P, G302V, and G325E are defective for ded1$1 D 5$ complementation at the semi-permissive temperature of $33^{\circ} \mathrm{C}$. The DDX3X P568L mutant shows only a minor defect in Ded 1 complementation at $33^{\circ} \mathrm{C}$. The $\mathrm{K} 230 \mathrm{~A}$ ATPase defective mutant of DDX3X is defective in Ded 1 complementation at both $25^{\circ} \mathrm{C}$ and $33^{\circ} \mathrm{C}$.

Source: Reprinted with permission from Elsevier Inc. Epling, L.B., C.R. Grace, B.R. Lowe, J.F. Partridge, and E.J. Enemark, Cancer-associated mutants of RNA helicase $D D X 3 X$ are defective in RNA-stimulated ATP hydrolysis. J Mol Biol, 2015. 427(9): p. 1779-96. Creative Commons license: https://creativecommons.org/licenses/by/4.0/. DOI: 10.1016/j.jmb.2015.02.015. 
A.
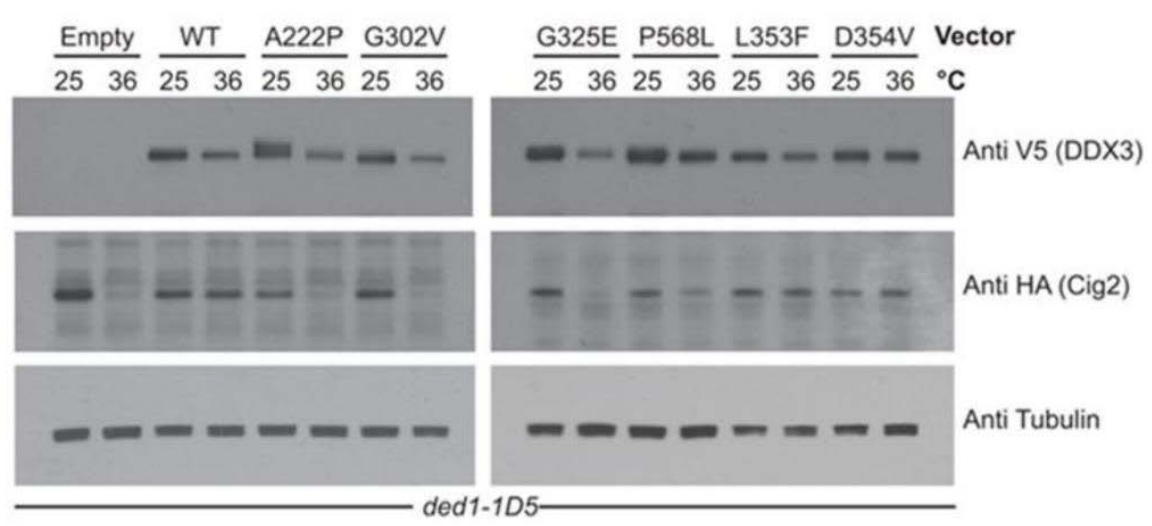

B.

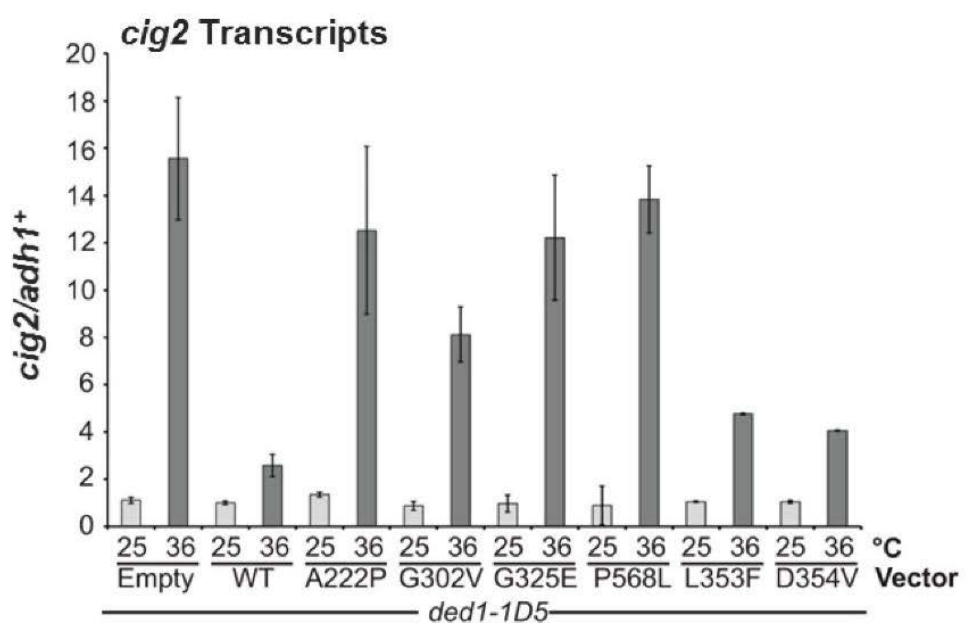

Figure 5-3. Defects in ded1-1D5 complementation by specific DDX3X mutants correlate with decreased Cig2 protein levels.

A. Western blot to monitor Cig2-3x HA levels in ded1-1D5 strain expression the DDX3X mutants. The DDX3X mutants which cannot compliment for ded1-1D5 function at elevated temperatures (A222P, G302C, F325E, and P568L) show decreased Cig2 protein levels. B. $\operatorname{cig} 2^{+}$transcript levels normalized to the euchromatic $a d h 1^{+}$control were monitored at both $25^{\circ} \mathrm{C}$ and $36^{\circ} \mathrm{C}$ by qRT-PCR. No decrease in $\operatorname{cig} 2^{+}$transcription was observed in ded1-1D5 strains transformed with DDX3X mutants defective for complementation of Ded1 function at the $36^{\circ} \mathrm{C}$.

Source: Reprinted with permission from Elsevier Inc. Epling, L.B., C.R. Grace, B.R. Lowe, J.F. Partridge, and E.J. Enemark, Cancer-associated mutants of RNA helicase DDX3X are defective in RNA-stimulated ATP hydrolysis. J Mol Biol, 2015. 427(9): $\mathrm{p}$. 1779-96. Creative Commons license: https://creativecommons.org/licenses/by/4.0/. DOI: 10.1016/j.jmb.2015.02.015. 
$25^{\circ} \mathrm{C}$, ded1-61 function is perturbed. We then tested all ten DDX3X mutants in this background using the serial dilution assay in order to confirm the results from the analysis of the ded1-1D5 thermosensitive mutant (Figure 5-4A). In agreement with the previous data, A222P, G302V, and G325E could not complement the ded1-61 coldsensitive defect at the non-permissive temperature. In contrast to the ded1-1D5 background, the protein levels of non-functional mutants increased in the ded1-61 background, suggesting that the defects previously observed were not due to decreased DDX3X protein levels. The results showed consistency between the thermosensitive mutants, with G302V, A222P, G325E, and K230A all having severe growth defects at temperatures where Ded1 is nonfunctional, while P568L only showed a minor growth defect in the ded1-1D5 background. Taken together, this indicates that the G302V, A222P, G325E, and P568L mutations of DDX3X produce a protein which cannot fully complement for Ded1 function.

\section{Normal DDX3X Function Is Required for Cig2 Translation}

It is known that the ded1-1D5 mutant causes a global decrease in translation with more severe defects in the translation of specific transcripts, including two B-type cyclins, Cig2 and Cdc13. These transcripts both have long complex 5' and 3' UTRs [162]. Since Ded1 is required for proper translation of Cig2 and Cdc13, we hypothesized that the growth deleterious mutants of DDX3X would also affect the translation of these transcripts.

We first tested whether DDX3X could properly complement for the role of Ded1 in the translation of B-type cyclins. DDX3X and Ded1 vector constructs were transformed into ded1-1D5 with either Cig2-3x HA or Cdc13-3x HA in order to monitor Cig2 and Cdc13 protein levels. Levels of Cdc13-3x HA could not be detected in our assay so we focused solely on Cig2-3x HA. To monitor Cig2 protein levels, a temperature shift assay was performed. In this assay, cells were grown overnight at the permissive temperature of $25^{\circ} \mathrm{C}$ before splitting the cultures and incubating either at the permissive $\left(25^{\circ} \mathrm{C}\right)$ or non-permissive temperature $\left(36^{\circ} \mathrm{C}\right)$ for an additional 4 hours. WCEs were prepared and protein levels of Cig2-3X HA were examined. ded1-1D5 cells grown at the non-permissive temperature have decreased Cig2 protein levels, which we reproduced in our experiment (Figure 5-1D) [162]. In contrast, ded 1-1D5 cells transformed with WT Ded1 or DDX3X showed no decrease in Cig2 protein after incubation at the non-permissive temperature, indicating that DDX3X can rescue the decrease in Cig2 protein levels associated with loss of Ded1 function.

In order to determine if any of the cancer associated mutants were defective for complementing the role of Ded1 in Cig2 translation, the same temperature shift experiment was performed on ded1-1D5 cells containing A222P, G302V, G325E, P568L, L353F, or D354V DDX3X mutants (Figure 5-3A). The L353F and D354V mutants which can complement for Ded1 function at elevated temperatures showed no change Cig2 protein levels. In contrast, the DDX3X A222P, G302V, G325E, and P568L mutants which cannot complement for the ded1-1D5 defect at elevated temperatures showed 
A.

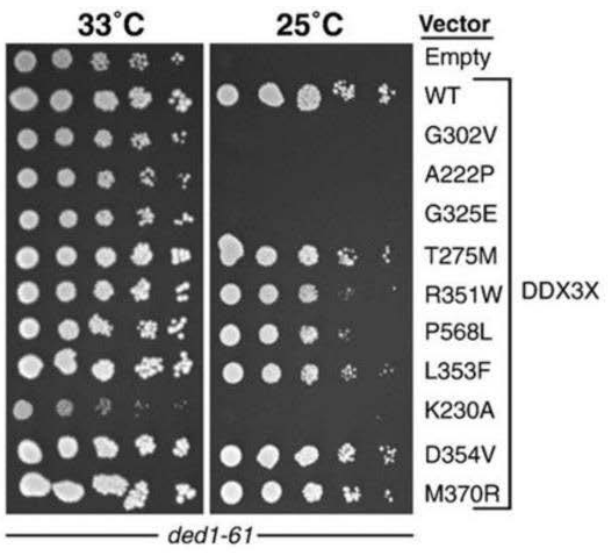

B.

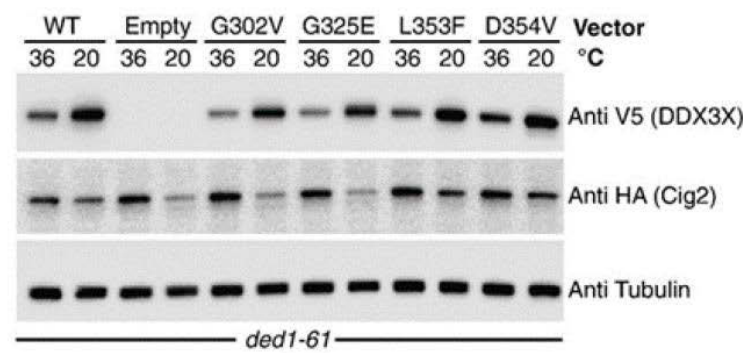

C.

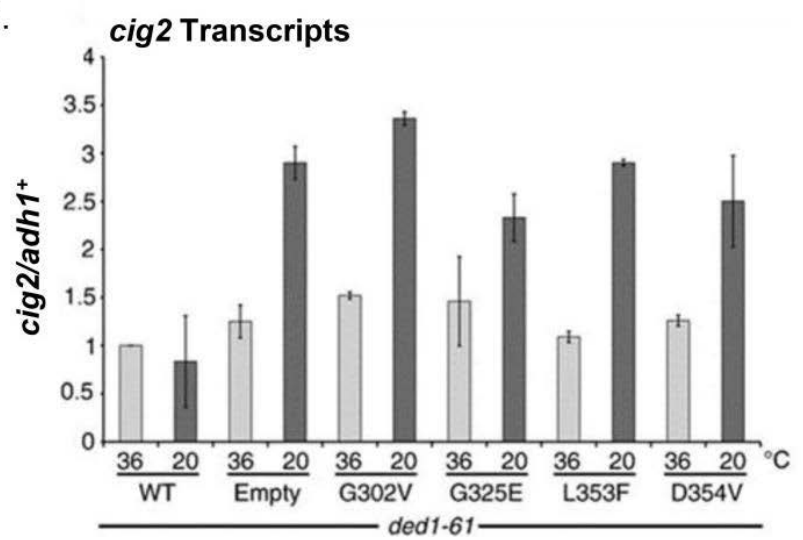

Figure 5-4. Specific DDX3X mutants cannot complement the ded1-61 temperature sensitive defect.

A. Five-fold serial dilution of episomal DDX3X mutants transformed into the coldsensitive ded1-61 background. A222P, G302V, and G325E are defective for ded1-61 complementation at the semi-permissive temperature of $25^{\circ} \mathrm{C}$. B. Western blot to monitor Cig2-3x HA levels in ded1-61 strain expression the episomal DDX3X mutants. The DDX3X G302V and G325E mutants which cannot compliment for ded1-61 function at $25^{\circ} \mathrm{C}$ show decreased Cig2 protein levels at the non-permissive temperature of $20^{\circ} \mathrm{C}$. C. cig $2^{+}$transcript levels normalized to the euchromatic $a d h 1^{+}$control were monitored at both $36^{\circ} \mathrm{C}$ and $20^{\circ} \mathrm{C}$ by qRT-PCR. No decrease in $\operatorname{cig} 2^{+}$transcription was observed in ded1-61 strains expressing DDX3X mutants defective for complementation at $20^{\circ} \mathrm{C}$.

Source: Reprinted with permission from Elsevier Inc. Epling, L.B., C.R. Grace, B.R. Lowe, J.F. Partridge, and E.J. Enemark, Cancer-associated mutants of RNA helicase $D D X 3 X$ are defective in RNA-stimulated ATP hydrolysis. J Mol Biol, 2015. 427(9): p. 1779-96. Creative Commons license: https://creativecommons.org/licenses/by/4.0/. DOI: 10.1016/j.jmb.2015.02.015. 
decreased protein levels of Cig2 after incubation at $36^{\circ} \mathrm{C}$. Since the levels of DDX3X were decreased at elevated temperatures, we confirmed this result using the ded1-61 cold sensitive mutant cells containing the G302V, G325E, L353F, or D354V mutants of DDX3X (Figure 5-4B). Consistent with the ded1-1D5 results, DDX3X L353F and D354V showed no defect in Cig2 protein levels after being incubated at the nonpermissive temperature of $20^{\circ} \mathrm{C}$. In contrast, the G302V and G325E mutants showed decreased protein levels of Cig2 after the incubation, consistent with the results of the ded1-1D5 transformants. This data indicates that the A222P, G302V, G325E, and P568L mutants of DDX3X cannot complement for the role of Ded1 in Cig2 translation.

To rule out the possibility that the DDX3X A222P, G302V, G325E, and P568L mutants are affecting transcription of Cig2 rather than its translation, we performed additional temperature shift experiments and prepared total RNA from cells in order to make cDNA. This allowed for the examination of the level of Cig2 transcripts by qRTPCR. In the ded1-1D5 background, after shifting the culture to $36^{\circ} \mathrm{C}$, there was an increase in the levels of Cig2 transcripts in all cells (Figure 5-3B). The DDX3X A222P, G302V, G325E, and P568L mutants, which showed decreased Cig2 protein levels, expressed the highest levels of Cig2 transcripts. It is possible that the increased Cig2 transcription is due to G1 arrest of the non-complementing mutants, as Cig2 transcription is cell cycle regulated. This experiment was repeated in the dedl-61 cold-sensitive background and Cig2 transcript levels similarly showed no decrease in the DDX3X A222P, G302V, G325E, and P568L mutants which exhibit low Cig2 protein levels (Figure 5-4C). These results show that reduced Cig2 protein levels in some DDX3X mutant backgrounds is not caused by transcriptional defects and that these mutations may lead to defective Cig2 translation. 


\section{CHAPTER 6. DISCUSSION AND FUTURE DIRECTIONS}

\section{The NTD and HDAC Activity of Sir2 Are Necessary for Establishment}

Proper heterochromatin formation in fission yeast involves three distinct steps beginning with heterochromatin establishment, in which de novo deacetylation and methylation events occur. The necessity for RNAi in this process remains unclear, although we believe the evidence points to RNAi independent de novo deposition of $\mathrm{H} 3 \mathrm{~K} 9 \mathrm{me}$ by Clr4. This leads to the recruitment of factors involved in the second step, in which heterochromatin is spread over the adjacent area in an RNAi dependent manner. Once heterochromatin has been established and spread over appropriate domains, heterochromatin must be maintained as cells divide. While most research regarding the role of Sir2 at heterochromatic loci has focused mainly on heterochromatin maintenance, recent research has shown a role for Sir2 in heterochromatin establishment by deacetylation of $\mathrm{H} 3 \mathrm{~K} 9$, which is a prerequisite for the methylation of $\mathrm{H} 3 \mathrm{~K} 9$ [82, 83]. Although we know Sir2 is required for heterochromatin establishment at centromeres, the exact nature of this process remains unclear.

\section{The NTD of Sir2 Is Required for Proper Establishment}

The current understanding of the role of Sir2 in the establishment of heterochromatin is limited. Until recently, Sir2 was mainly recognized for its function at telomeres and mat. While Sir2 is required to establish pericentromeric heterochromatin, it is unclear whether its role is solely dependent on HDAC activity since the Sir2 N247A catalytic mutant exhibits a minimal level of heterochromatin establishment at the centromere [83]. Here we dissected the role of Sir2 in heterochromatin establishment, separating the function of the HDAC activity from other domains of Sir2. This allowed for the identification of the Sir2 NTD as a critical domain important for proper heterochromatin establishment, possibly independent of full Sir2 HDAC activity.

Further dissection of the Sir2 NTD led us to focus on a serine rich patch stretching from residues 23 to 36 within the Sir2 NTD. Deletion of the serine rich patch in the Sir2 N247A mutant (Sir2 $\Delta 23-36$ N247A and Sir2 $\Delta 1-36$ N247A) led to a complete loss of de novo centromeric heterochromatin establishment in contrast to limited heterochromatin formation exhibited by Sir2 N247A transformants. In contrast, Sir2 $\Delta 1$ 20 N247A transformants could establish low levels of heterochromatin similar to Sir2 N247A transformants. This data confirmed the serine rich patch is required for de novo heterochromatin formation in the absence of HDAC activity. When we dissected the serine rich patch, the establishment defect correlates with the SSSG repetitive region between residues 27 and 34. If this repetitive region is left intact, Sir2 N247A transformants exhibit low levels of centromeric heterochromatin establishment, but partial deletion of this region leads to a complete loss of establishment. Chromatin association data indicates that the NTD region of Sir2 along with HDAC activity is important for Sir2 recruitment to chromatin, as deletion of the serine rich region or 
mutating N247 decreases recruitment. The defect in recruitment is not due to impaired nuclear localization, as deletion of the NTD does not exclude Sir2 from the nucleus. This leads to the conclusion that the Sir2 NTD and HDAC activity are both important for the recruitment of Sir2 to chromatin and hence, the de novo establishment of heterochromatin. Mutation of both causes a complete loss of heterochromatin establishment at the centromere.

Due to the serine rich nature of this region of the Sir2 NTD, we examined PTMs of Sir2. MS/MS analysis indicates that S23, S25, and S27 can be phosphorylated. Although phosphorylation of these residues occurs in vivo, no effect on centromeric heterochromatin esablishment was observed when phosphorylation and dephosphorylation mimetics were combined with the N247A mutation. Only S27 lies within the SSSGSSG repetitive region important for establishment, and due to the repetitive nature of this region, it is possible that neighboring serines can be phosphorylated when S27 is mutated. Further investigation will be required to fully understand the effects of Sir2 NTD phosphorylation.

Our analysis of the Sir2 NTD in the N247A background led us to two possible models for its function (Figure 6-1). One possibility is that the Sir2 N247A mutant retains low levels of HDAC activity and the NTD may stimulate this activity. By deleting the NTD in the N247A background, all Sir2 HDAC activity may be lost. In this scenario, the limited activity might be sufficient to deacetylate H3K9 and H3K14 at low levels, corresponding to the minimal heterochromatin establishment observed. PTM of the Sir2 NTD could play a role in regulating HDAC activity by affecting interactions between the unstructured Sir2 NTD and the catalytic core or altering basic protein structure. However, this seems unlikely since our in vitro and in vivo data suggest that Sir2 $\Delta 1-129$ has fully functional HDAC activity. In contrast, Sir2 N247A appears defective for HDAC activity in vitro and in vivo [83]. Although the preliminary evidence suggests the NTD does not regulate HDAC activity, further study will be needed to completely rule out this scenario.

Alternatively, the NTD may be necessary to recruit factors which can establish low levels of heterochromatin independent of Sir2 HDAC activity. In this scenario, Sir2 could be serving as a scaffold to recruit another HDAC in addition to its own deacetylase activity. One possibility would be an interaction with $\mathrm{Clr} 3$, which could create an environment sufficient for Clr4 to methylate $\mathrm{H} 3 \mathrm{~K} 9$ and low levels of de novo heterochromatin formation to occur. Another interesting possibility involves Sir 2 serving as a scaffold to directly recruit $\mathrm{Clr} 4$ to chromatin where it can methylate $\mathrm{H} 3 \mathrm{~K} 9$ in an RNAi independent manner. Although attempts to identify possible Sir2 interaction partners were unsuccessful, we believe it is still likely that Sir2 is guided to heterochromatic loci through interactions with other proteins. Additional purification experiments using endogenous tagged Sir2 proteins may identify such interactors.

Unlike most enzymes, HDACs do not have well defined target motifs allowing for substrate recognition. Instead, almost all HDACs are found in complex with other non-enzymatic proteins which contain domains necessary for deacetylation specificity [209]. This allows for HDAC activity to be guided by proximity rather than recognition 
A.

Regulation of HDAC activity?

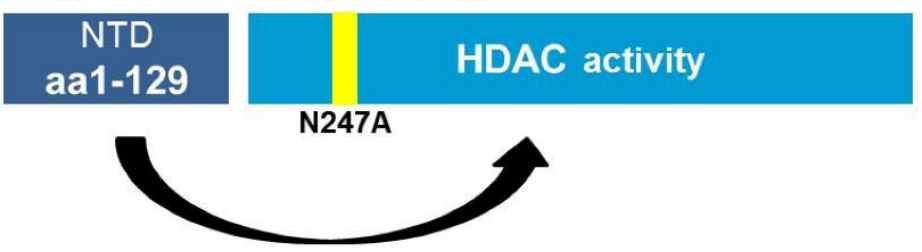

B. Function independent of HDAC activity?

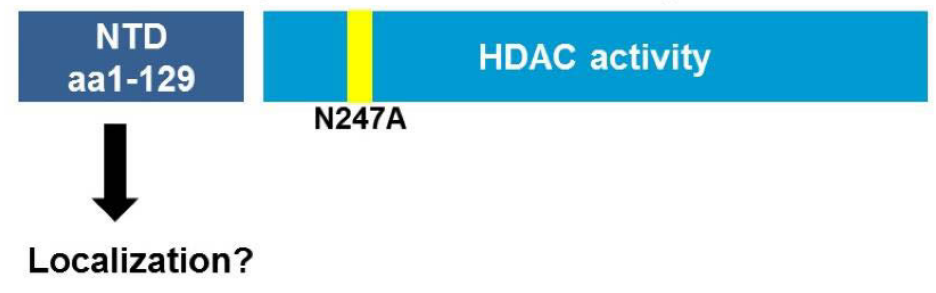

Recruitment of other factors?

Figure 6-1. Possible roles of the Sir2 NTD.

A. The NTD may positively regulate the HDAC activity of Sir2, allowing for minimal HDAC activity in the Sir2 N247A background which is absent when the NTD is deleted. B. The NTD of Sir2 could aid in the recruitment of other factors to heterochromatin, which may be necessary for initial de novo $\mathrm{H} 3 \mathrm{~K} 9$ me by $\mathrm{Clr} 4$ independent of the HDAC activity of Sir2. 
of a specific sequence motif. Adding to the complexity, HDAC often contain extensive PTMs which can affect localization, interaction with members of a complex, and selfmultimerization [96]. Understanding the involvement of the NTD of Sir2 independent of HDAC activity and how Sir2 is recruited to chromatin will require an understanding of the localization, interacting partners, and PTMs of Sir2 which we have begun to dissect in this study.

\section{Potential Sir2 Interactors: Insight from Sir2 Homologs}

Since nearly all HDACs have interacting partners necessary for targeting, it is likely that Sir2 is no different. To date, no interactors or complex in which Sir2 acts have been identified though MS/MS analysis. If Sir2 does interact with other proteins to promote RNAi independent heterochromatin formation, it is likely that this interaction is mediated through the large NTD and CTD. Unfortunately, these domains contain little structure and share only minimal homology with Sir2 homologs, making it difficult to correlate interacting partners between the homologs. Since we have uncovered a novel contribution of the Sir2 NTD in its recruitment to chromatin and de novo heterochromatin formation, the possibility of the NTD serving as an interaction domain remains intriguing. Although we were unable to determine any potential interaction mediated through this domain, studies involving Sir2 homologs may offer insight.

In budding yeast, the SIR complex is necessary for heterochromatic silencing at the mating type and telomeres. The complex consists of Sir2p, Sir3p, and Sir4p in a 1:1:1 stoichiometric ratio. Both Sir3p and Sir4p aid in the recruitment of Sir2p through interactions with nucleosomes and the DNA binding proteins Rap1p, Abflp, and Orc1p along with another Sir protein, Sir1p [210, 211]. It is thought that Sir3p can bind to these regions and recruit the Sir $2 p / \operatorname{Sir} 4 p$ sub-complex allowing for the formation of the entire SIR complex $[111,211]$. Sir2p interacts with Sir4p within the NTD of Sir2p close to the catalytic core and Sir4p binding serves to allosterically stimulate Sir2p HDAC activity by stabilizing the NTD and catalytic core [212]. The recruitment of the SIR complex to telomeres and mating type silencers allows for the nucleation of heterochromatin. Once at these sites, Sir2p can deacetylate H4K16 on adjacent nucleosomes. This, along with oligomerization of Sir3p allows for further SIR recruitment and spreading of heterochromatin $[112,213]$. This model fits well with the observation that both the HDAC activity and NTD of fission yeast Sir2 impact its recruitment to chromatin and are necessary for de novo heterochromatin establishment. Unfortunately, no known homologs of Sir3p and Sir4p exist in fission yeast and there is only minimal conservation between the NTD of Sir2 in budding and fission yeasts. It remains possible that fission yeast Sir2 behaves similarly with proteins which have yet to be identified, but current purifications cast doubt on any 1:1 stoichiometric complexes existing in fission yeast.

Like the fission yeast Sir2, human SIRT1 has a conserved HDAC core surrounded by a large NTD and CTD. The most studied role of human SIRT1 is its role in metabolic regulation through regulation of gene expression as well as deacetylation of non-histone proteins. SIRT1 has also been linked to cancer through its deacetylation of $\mathrm{p} 53$, altering 
its tumor suppressor activity [214, 215]. In fission yeast, the ability of Sir2 to deacetylate non-histone proteins has not been examined. Like Sir2, SIRT1 has been shown to deacetylate $\mathrm{H} 3 \mathrm{~K} 9$ and H4K16, suggesting a conserved role between the enzymes [216].

In humans, the centromere is surrounded by complex repetitive elements known as $\alpha$-satellite repeats where heterochromatin assembles via $\mathrm{H} 3 \mathrm{~K} 9 \mathrm{me}$ and binding of $\mathrm{HP} 1$ proteins. Although the steps in the assembly of human pericentromeric heterochromatin are not clear, fission yeast provide insight into how this process may occur due to the conservations of key proteins [72, 217]. It has been shown that SIRT1 can interact with histone $\mathrm{H} 1$, deacetylating the histone and aiding in its recruitment to establish heterochromatin [216]. Although fission yeast has no true $\mathrm{H} 1$ homolog, this observation is consistent with experiments showing Sir2 homologs in other organisms can interact with nucleosomes in vitro, raising the possibility that Sir2 may directly interact with histones $[212,216]$. Although our Sir2 MS/MS analysis showed interactions with several histones, the interactions were not conclusive. Further experiments will be needed to investigate validity of this observation.

Interestingly, human SIRT1 can also interact with and regulate the activity of the H3K9 HMT SUV39H1. The interaction between SIRT1 and SUV39H1 occurs through the NTD of SIRT1 and is thought to stimulate SIRT1 activity. SIRT1 also regulates the activity of SUV39H1 by deacetylation of a lysine within its catalytic SET domain. Deacetylation of this residue was shown to increase methyltransferase activity, likely by stabilizing domain interactions with SUV39H1 [118]. It is possible that fission yeast Sir2 is recruiting $\mathrm{Clr} 4$ to heterochromatic regions and regulating the activity of $\mathrm{Clr} 4$ in addition to deacetylation of $\mathrm{H} 3 \mathrm{~K}$ 9. This would explain how $\mathrm{H} 3 \mathrm{~K} 9$ me could be deposited in an RNAi independent manner by providing an alternative mechanism of Clr4 recruitment. This could also explain the low levels of establishment observed in the sir2 $N 247 A$ strain, where Clr4 can methylate H3K9 which is not already acetylated allowing, for a minimal level of establishment to occur. In this scenario, the HDAC activity of Sir2 would only be required to deacetylate neighboring $\mathrm{H} 3 \mathrm{~K} 9$ residues to allow for heterochromatic spreading. Unfortunately, no experiments have been able to demonstrate that an interaction exists between Sir2 and Clr4 [unpublished communication from Janet Partridge 03/03/2017]. The exact site of acetylation is not conserved in Clr4 and we previously mutated a similar site on Clr4, but no impact on heterochromatin assembly was observed [unpublished communication from Janet Partridge, 03/03/2017]. It remains possible that a connection between Sir 2 and Clr4 exists, but only under certain conditions since Sir2 is likely sensitive to cell stress.

\section{PTM of Sir2}

Our findings highlight the importance of the serine rich patch within the NTD of Sir2 as having a role in pericentromeric heterochromatin establishment, with the SSSG repeat within the serine rich patch being important for establishment. MS/MS indicated that phosphorylation occurs on S23, 25, and 27 within the serine rich patch. Although mutating these residues to mimic phosphorylation or dephosphorylation did not affect 
heterochromatin establishment, it is possible that PTM within this region may still be important for Sir2 function, with specific combinations of phosphorylation in this region affecting Sir2 function. Regulation of Sir2 by phosphorylation would be consistent with homologs of Sir2 in higher eukaryotes.

SIRT1 undergoes extensive phosphorylation within its large NTD and CTD which affects its stability, localization, and activity. The first comprehensive study on phosphorylation of SIRT1 by MS/MS analysis indicated that SIRT1is phosphorylated on 13 sites within these domains, with no phosphorylation detected in the HDAC domain. Two sites within the CTD are phosphorylated by $\mathrm{CyclinB} / \mathrm{Cdk} 1$, a complex which acts at the G2/M phase transition. Loss of SIRT1 phosphorylation at these sites leads to defective G2 entry and proliferation defects [123]. Loss of all SIRT1 phosphorylation led to decreased HDAC activity although no decrease in HDAC activity was observed in vitro when specific phosphorylation sites were mutated. The cause of this cell cycle block in S phase remains unclear [123]. Phosphorylation of SIRT1 by CK2 within the NTD and CTD leads to increased HDAC activity and cell survival due to increased p53 deacetylation, which causes decreased p53 activity [128]. SIRT1 phosphorylation by DYRK1A and DYRK3 within the CTD had a similar effect, leading to increased deacetylation of p53 and increased cell survival [127]. Research also suggests that CTD phosphorylation may keep SIRT1 in a monomeric conformation during stress which promotes higher SIRT1 activity and increased cell survival, while non-phosphorylated SIRT1 forms oligomers resulting in decreased SIRT1 activity [218]. Phosphorylation of the SIRT1 NTD by JNK1 leads to increased nuclear localization, increased HDAC activity, and increased specificity towards deacetylation of histone H3, while JNK2 dependent phosphorylation of SIRT1 increased protein stability [124, 126].

While many of these signaling pathways are conserved in fission yeast, no links between Sir2 and these kinases are currently known. Our work shows that PTM of the Sir2 NTD does occur and links between SIRT1 regulation in mammals and Sir2 regulation in fission yeast may exist. PTM of Sir2 homologs in higher eukaryotes could aid in explaining the importance of the serine rich region of Sir2 in establishment, although our current in vitro and in vivo examination of HDAC activity argues against a role in the modulation of HDAC activity by the Sir2 NTD.

If HDAC activity is not influenced by these modifications, another interesting possibility is that the Sir2 NTD PTM may be influencing the binding of Sir2 interactors. Currently, we have MS/MS data indicating Sir2 can interact with the 14-3-3-like proteins $\operatorname{Rad} 24$ and $\operatorname{Rad} 25$. Like mammalian 14-3-3 proteins, $\operatorname{Rad} 24$ and $\operatorname{Rad} 25$ bind to phosphorylated serines and threonines, playing a role in multiple signaling processes within the cell. In mammalian systems, 14-3-3 proteins are known to regulate class II HDACs by altering nuclear localization [219]. Since deletion of the Sir2 NTD does not impact nuclear localization, if Sir2 interacts with Rad24 and Rad25, modulation of Sir2 localization is not the purpose of such an interaction. In Caenorhabditis elegans, the Sir2 orthologue SIR2.1 interacts with 14-3-3 proteins during stress to promote complex formation with the transcription factor DAF-16 [220, 221]. It remains possible that under certain conditions or points in the cell cycle, specific PTMs Sir2 promote interaction with 
$\operatorname{Rad} 24$ and $\operatorname{Rad} 25$. In fission yeast, $\operatorname{Rad} 24$ was shown to be associated with Rik1 of the Clr-C complex in vivo, and in vitro experiments suggest $\operatorname{Rad} 24$ and $\operatorname{Rad} 25$ can associate with Agol of RITS, providing possible links between components of heterochromatin assembly and $\operatorname{Rad} 24$ and $\operatorname{Rad} 25$ [222, 223]. In humans, $14-3-3 \varepsilon$ and $14-3-3 \zeta$ have also been shown to interact with histone $\mathrm{H} 3$. These proteins have an affinity for $\mathrm{H} 3$ tails containing phosphorylation of $\mathrm{H} 3 \mathrm{~S} 10$, along with acetylation at $\mathrm{H} 3 \mathrm{~K} 9$ or $\mathrm{H} 3 \mathrm{~K} 14$ [224, 225]. If $\operatorname{Sir} 2$ indeed binds $\operatorname{Rad} 24$ and $\operatorname{Rad} 25$, it is possible that they aid in the initial recruitment of Sir2 to heterochromatic regions either through interactions with proteins involved in heterochromatin formation or binding to histone H3. While the lack of an establishment defect in the rad24 $\Delta$ strain would argue against the importance of 14-3-3 proteins in heterochromatin establishment, it is possible that Rad25 can compensate for Rad24 masking any observable phenotype.

It is possible that the interactions and PTMs of sirtuins from other systems give clues about potential interactor and regulatory events of Sir2. Unfortunately, much of the functions of PTM regulation of SIRT1 remains unclear and homologs of key budding yeast Sir2p interactors are not found in fission yeast, clouding our understanding of how $\mathrm{Sir} 2$ is recruited and regulated. It is possible that Sir2 does not act in one solely defined complex but could act in cooperation with distinct proteins depending on cell cycle timing or factors causing cell stress. The PTM of the NTD of Sir2 adds an extra layer of complexity to this idea, as these modifications may have a direct impact on the interaction of Sir2 with other proteins. Although we were unable to demonstrate that these specific sites of phosphorylation were important in heterochromatin establishment, it is possible that other sites exist which we did not detect and certain combinations of phosphorylation are necessary for heterochromatin establishment.

\section{SHREC Can Function as Independent Modules}

We know that Sir2 and Clr3 perform overlapping functions in the maintenance of centromeric heterochromatin. Our current understanding depicts a situation where Sir2 is required for $\mathrm{H} 3 \mathrm{~K} 9$ deacetylation and $\mathrm{Clr} 3$ is required for $\mathrm{H} 3 \mathrm{~K} 14$ deacetylation creating an environment which is favorable for $\mathrm{Clr} 4$ recruitment and $\mathrm{H} 3 \mathrm{~K} 9 \mathrm{me}$ [83]. Although the exact mechanism of this cooperation is not well understood, cooperation with SHREC is also likely to be key in heterochromatin establishment.

Although SHREC affects nucleosome occupancy, individual subunits of the complex show varying effects at individual genomic loci. In fission yeast, five classes of nucleosome free regions (NFRs) were identified in $\operatorname{clr} 4 \Delta$ strains. While clr $3 \Delta$ and chp $2 \Delta$ have large effects on nucleosome positioning, the effects of mit $1 \Delta$ are restricted to specific loci within the genome. Within the different classes of NFRs, Clr3 and Chp2 can act independently of one another in their broad roles [102]. This analysis fits well with our RNA-seq analysis of SHREC components, which shows distinctive transcriptional profiles between different SHREC components. The $\operatorname{clr} 1 \Delta$ and $\operatorname{clr} 2 \Delta$ showed the most overlap, also sharing a high degree of overlap with $\operatorname{clr} 3 \Delta$. Similarly, mit $1 \Delta$ and chp $2 \Delta$ shared a high degree of overlap. Very little overlap in regulation was observed between 
all SHREC subunits [149]. Based on our RNA-seq analysis and previous nucleosome positioning data, we believe SHREC can function in distinct modules: a remodeler module which includes Mit1 and Chp2 and an HDAC module which includes Clr1, Clr2, and $\mathrm{Clr} 3$. These modules likely have distinct genetic targets.

In the structure of SHREC, Clr1 provides the scaffold which bridges Mit1 and Chp2 through its N-terminus and with Clr2 and Clr3 through its CTD. Subsequent work has shown that overexpression of truncated Clr1 (Clr1T) lacking the region necessary for binding Mit 1 and Chp2 but retaining the zinc fingers of Clr1 and sites of Clr2 and Clr3 interaction can complement for the loss of centromeric silencing observed in a $\operatorname{clr} 1 \Delta$. In contrast, deletion of the Clr1 interface with Clr2 and Clr3 cannot fully complement the defect observed in a clrl $\Delta$ strain. This corroborates our RNA-seq data, suggesting that under certain situations, sub-complexes of SHREC can function independently of one another in transcriptional regulation and heterochromatin assembly. While $\mathrm{Chp} 2$ recruits the remodeler module by binding $\mathrm{H} 3 \mathrm{~K} 9$ me through its $\mathrm{CD}$, the HDAC module is recruited through $\mathrm{Clr} 2$, which can bind to nucleic acid thought its Methyl-CpG bindinglike domain (MBDL) [149].

\section{Sir2 Shows Overlap with the SHREC Remodeler Module}

When the transcriptional profile of sir $2 \Delta$ cells was compared to the deletion of individual SHREC components, a high degree of overlap with $\operatorname{chp} 2 \Delta$ and mit1 $\Delta$ was observed. This data indicates that Sir2 exhibit overlaps in transcriptional regulation with the SHREC remodeler sub-complex in addition to its redundancy with Clr3 in centromeric heterochromatin maintenance. Supporting this, we show genetically that Sir2 appears to be acting in the same pathway as Chp2 and Mit1 since sir $2 \Delta$ mit $1 \Delta$ and $\operatorname{sir} 2 \Delta$ chp $2 \Delta$ double mutant strains do not show significant additive defects in silencing at loci which showed overlapping transcriptional regulation in the RNA-seq profiling. In contrast, the phenotype of $\operatorname{sir} 2 \Delta$ combined with $\operatorname{clr} 1 \Delta, \operatorname{clr} 2 \Delta$, or $\operatorname{clr} 3 \Delta$ is additive at these loci, indicating they are likely acting through different pathways. This data suggests that Sir2 and Clr3 may be able to compensate for one another in the transcriptional regulation of specific loci, consistent with their redundancy in heterochromatin maintenance. Currently, these findings are preliminary and more work will be needed to address the relevance their relevance.

In humans, connections between SIRT1 and NuRD have also been made. SIRT1 can physically interact with HDAC1 of NuRD and can deacetylate HDAC1 to stimulate its HDAC activity in neurons [226]. HDAC1 is a component of the NuRD complex which provide part of its histone deacetylase function similar to Clr3 of SHREC. This is important in the context of dsDNA breaks where the HDAC activity of HDAC1 plays a role in non-homologous end joining pathway to repair these dsDNA breaks [226]. Also, acetylated HDAC1 has been shown to interact with heterochromatic regions and research indicates SIRT1 can regulate transcriptional repression mediated by HDAC1, suggesting connection between the HDACs in transcriptional regulation [227, 228]. Although no physical links between SHREC and Sir2 have been identified in fission yeast, it is 
possible that such a link may be conserved in fission yeast due to overlaps in transcriptional regulation.

Based on the transcriptional profiles of SHREC deletion strains and $\operatorname{sir} 2 \Delta$ strains, it is possible that histone deacetylation by Sir2 is necessary to create an environment favorable for the recruitment of the SHREC remodeler sub-complex. In sir2 $\Delta$ cells, the remodeler sub-complex may not be recruited to these loci, resulting in overlapping alterations of transcription observed by RNA-seq. This corresponds with the idea that deletion of Mit 1 affects nucleosome remodeling in regions not affected by deletion of Clr3 [102]. Alternatively, it could be theorized that under certain circumstances, Sir2 can interact with SHREC through the remodeler sub-complex. In humans, components of NuRD can interact with multiple proteins and complexes such as the interaction with SIRT1 described above [226, 229]. In establishment, an interaction between SHREC and Sir2, possibly through the NTD of Sir2, could be crucial to establish an environment devoid of $\mathrm{H} 3 \mathrm{~K} 9 \mathrm{ac}$ and $\mathrm{H} 3 \mathrm{~K} 14 \mathrm{ac}$, allowing for proper $\mathrm{Clr} 4$ recruitment and initial H3K9me events to occur. Further research will be needed to determine how Sir2 is acting in conjunction with the SHREC remodeler module in global transcriptional regulation.

\section{Future Directions for Sir2}

Based on our previous work, we know that Sir2 N247A mutant strain can establish low levels of heterochromatin [83]. This is dependent on the serine rich region of the Sir2 NTD which contains three sites of phosphorylation. Our data suggests this region does not impact the deacetylase activity of Sir2, but both the NTD and the HDAC activity of Sir2 are necessary for its recruitment to chromatin. Based on these findings, we suggest a model wherein Sir2 can interact with another component of heterochromatin assembly such as an HDAC, which could provide deacetylase activity in the presence of N247A. Alternately, association with Clr4 could facilitate methylation H3K9 in an RNAi-independent manner. These situations could lead to the minimal level of establishment observed in the sir2 N247A background, and the HDAC activity of Sir2 would likely be required to create an environment to further propagate heterochromatin. Although we were unable to demonstrate such interactions, it is likely that these complexes form only fleetingly or under certain conditions, possibly reliant on PTM of the NTD of Sir2 and which may be dependent on cell cycle timing or stress. This is supported by the fact that Sir2 homologs in other systems can act in response to changes in metabolism or cell stress [114, 230-233].

In the future, to identify components with which Sir2 interacts, we will likely have to synchronize cells in the cell cycle or induce cellular stress in order to examine Sir2 interactors. These same methods could also be employed to perform a detailed study of Sir2 PTM, as they may change depending on cellular conditions. Based on the RNAseq data, specific IP experiments could be performed to examine a possible association between Sir2 and the SHREC remodeler module. Also, we were unable to determine to what region of the genome Sir2 localizes based on our ChIP assay using episomally expressed Sir2. Our experiments to ChIP endogenously tagged Sir2 were unsuccessful. In 
order to determine where Sir2 normally localizes, experiments such as ChIP-Seq may be helpful to further characterize the role of Sir2 in heterochromatin establishment.

\section{Clr3 Can Deacetylate H2B}

Our work shows that $\mathrm{Clr} 3$ can deacetylate the $\mathrm{N}$-terminal tail of histone $\mathrm{H} 2 \mathrm{~B}$ in addition to its known target of $\mathrm{H} 3 \mathrm{~K} 14 \mathrm{ac}$. Our initial observation that an N-terminal H2B antibody cannot recognize $\mathrm{H} 2 \mathrm{~B}$ in $\operatorname{clr} 3 \Delta \mathrm{WCE}$ led us to examine the possibility that $\mathrm{Clr} 3$ can deacetylate lysines in the H2B NTD. The antibody used in the study recognizes an Nterminal peptide containing amino acids 1-16 of the fission yeast H2B N-terminus. Previous MS/MS analysis has shown that lysines within this region can be acetylate in vivo [152]. We hypothesized that increased acetylation within this region in $\operatorname{clr} 3 \Delta$ cells likely blocks the antibody recognition site, resulting in the decreased signal observed in clr3 $\triangle$ WCE.

To confirm that the N-terminus of $\mathrm{H} 2 \mathrm{~B}$ is deacetylated by $\mathrm{Clr} 3$ on sites within this region in a quantitative manner, we utilized MS/MS analysis of histones from $\operatorname{clr} 3 \Delta$ cells. Histones were purified by chromatin fractionation, acid extraction, and cation exchange to enrich for the positively charged histones, followed by two rounds of HPLC to separate the material based on hydrophobicity [195]. Using this method, purified histones were isolated which yielded good peptide coverage using MS/MS. The MS/MS analysis of histones purified from $\operatorname{clr} 3 \Delta$ cells versus WT cells indicated hyperacetylation of $\mathrm{H} 2 \mathrm{~B}$ $\mathrm{K} 5,6,10$, and 15, suggesting Clr3 can deacetylates these residues. We also showed that these residues can be acetylated by the HAT Gcn5. Antibodies were also generated against an $\mathrm{H} 2 \mathrm{~B} \mathrm{~N}$-terminal peptide acetylated on $\mathrm{K} 5,6,10$, and 15 which was used to further confirm that these marks exist in vivo and are enriched in clr3 $\Delta$ cells.

PTM of the H2B NTD has not been as well characterized as that of histones H3 and H4. In budding yeast, acetylation of lysines at positions conserved with the four sites of increased acetylation in fission yeast $c l r 3 \Delta$ strains were previously identified [234]. In $N$. crassa, lysines corresponding to $\mathrm{K} 5,6$, and 10 are also predicted to be deacetylated by the Clr3 homolog HDA1 [151]. In humans, two of the sites, K10 and 15, are conserved in all H2B isoforms and have been shown to be acetylated [235]. The conservation of these sites of acetylation indicates the possibility of a conserved function of $\mathrm{H} 2 \mathrm{~B}$ acetylation.

We hypothesized these marks may be important for heterochromatic silencing in fission yeast. When tested, we found that heterochromatin maintenance at the centromere, telomeres, and mat was not affected by mutations that mimic acetylation at these sites. We also showed that acetylation mimetics of these residues do not impact the establishment of centromeric heterochromatin. It is likely that the role of the mutations is more subtle, possibly in nucleosome positioning or chromatin spreading which was not investigated in this study. 


\section{H2B N-Terminal Tail Affects Transcription}

While histone $\mathrm{H} 3$ and $\mathrm{H} 4$ exhibit stronger interactions with DNA in the nucleosome, the N-terminal tail of $\mathrm{H} 2 \mathrm{~B}$ threads between the DNA gyres with several residues in the histone $\mathrm{H} 2 \mathrm{~B}$ repression domain (HBR) that stretches between aa30 and 37 interacting with the DNA backbone [1, 236, 237]. Although the lysines deacetylated by $\mathrm{Clr} 3$ do not lie in this region, it is still possible they may exert some influence on this process and alter chromatin compaction, a process in which $\mathrm{H} 2 \mathrm{~B}$ has previously been implicated, and decrease the charged based association with DNA [238]. This could lead to altered transcriptional regulation, a possibility we examined in this study. Our RNAseq analysis of the H2B 4Q acetyl mimetic cells indicates only minor transcriptional changes with no defining pattern. Consistent with this, in budding yeast, deletion of residues 4-37 of H2B leads to altered transcriptional regulation of over 700 genes and further mapping indicates that it is the HBR domain which is responsible for most of the transcriptional regulation properties of the $\mathrm{H} 2 \mathrm{~B}$ tail, with only minimal regulation by more upstream portions of the histone tail [237]. The HBR of H2B exhibits the strongest conservation within the N-terminal tail, with more variation in other tail regions, indicating the importance of this domain [239]. The budding yeast data correlates well with our transcriptional data from the fission yeast H2B 4Q mutant strains, which shows acetylation of $\mathrm{H} 2 \mathrm{~B} \mathrm{K5}, 6,10$, and 15 leads to only minimal changes in transcriptional regulation.

\section{H2B N-Terminal Tail Affects Apoptotic-Like Death}

In budding yeast, phosphorylation of H2B S10 by the Sterile 20 kinase has been shown to be associated with apoptotic-like cell death when cells are stressed by $\mathrm{H}_{2} \mathrm{O}_{2}$ treatment [240]. Before H2B S10 can be phosphorylated, H2B K11 must be deacetylated by the HDAC Hos3. This suggests that H2B K11 acetylation status serves as a regulator of apoptotic-like cell death in budding yeast upon stress [241]. This is similar to higher eukaryotes, in which H2B S14 phosphorylation is associated with apoptosis [242]. In higher eukaryotes, research suggests deacetylation of the neighboring H2B K15 site is a prerequisite to obtain phosphorylation of H2B S14 [243]. Although fission yeast can undergo apoptotic like death in response to cellular stress and both these modification sites are conserved, no study has examined this phenomenon, likely because fission yeast have a single copy of $\mathrm{H} 2 \mathrm{~B}$ making mutational studies challenging. It is possible that mutating K11 to an acetyl mimetic could affect cell survival phenotypes caused by other acetylation sites and we did not address this in our study since $\operatorname{clr} 3 \Delta$ cells are not sensitive to $\mathrm{H}_{2} \mathrm{O}_{2}$ [244].

\section{Role of Clr3 in Nucleosome Stability}

Although SHREC plays a large role in nucleosome positioning, research has shown that the role of $\mathrm{Clr} 3$ in this process extends beyond H3K14 deacetylation. H3K14 deacetylation by $\mathrm{Clr} 3$ is hypothesized to prevent the recruitment of the essential 
chromatin structure remodeling complex (RSC) important in NFR formation [102]. In fission yeast, $\mathrm{Clr} 3$ has a role in preventing nucleosome turnover at centromeric regions by blocking remodeling complexes. This allows for stable inheritance of heterochromatic regions, possibly by retaining histones with the PTMs necessary to reassemble heterochromatin [150]. Uncatalyzed sliding of nucleosomes has also been observed in the absence of the $\mathrm{H} 2 \mathrm{~B} \mathrm{~N}$-terminal tail [245]. It is possible that deacetylation of $\mathrm{H} 2 \mathrm{~B} \mathrm{K5}, 6$, 10 , and 15 by $\mathrm{Clr} 3$ provide additional targets important for the role of $\mathrm{Clr} 3$ in nucleosome positioning and epigenetic inheritance of heterochromatin. It has been shown that deletion of $g c n 5^{+}$and $m s t 2^{+}$, which acetylate H3K14, cannot full bypass the nucleosome loss observed in clr $3 \Delta$ cells, suggesting other targets of $\mathrm{Clr} 3$ are important in nucleosome stability [145]. It is possible that deacetylation of $\mathrm{H} 2 \mathrm{~B}$ by Clr3 may be important in nucleosome positioning, possibly by blocking the recruitment of remodeling factors or strengthening nucleosomal interaction with DNA to prevent the formation of NFRs at certain heterochromatic loci.

\section{Future Directions for H2B Acetylation Study}

Our work confirmed that $\mathrm{Clr} 3$ can deacetylate H2B K5, 6, 10, and 15 in vivo. To further confirm this observation, we will test whether $\mathrm{Clr} 3$ can deacetylate acetylated $\mathrm{H} 2 \mathrm{~B}$ peptides in vitro. We also plan to use our newly generated antibodies against $\mathrm{H} 2 \mathrm{~B}$ acetylation at these sites to perform ChIP experiments, allowing us to determine if lack of this modification is restricted to heterochromatic regions relative to euchromatic regions. This will allow us to further characterize the importance of these PTMs as targets of Clr3 in heterochromatin assembly.

We also plan to examine nucleosome turnover and occupancy in the presence of $\mathrm{H} 2 \mathrm{~B}$ acetylation mimetics. Although we see no broad defects in silencing at heterochromatic loci, it is possible that defects in histone turnover or nucleosome positioning exist. We will use in vivo PCR-based nucleosome scanning as previously described to examine loci at which Clr3 is necessary to form NFRs [145]. We will also examine the effects of these mutations on nucleosome turnover since $\mathrm{Clr} 3$ has a role in preventing nucleosome turnover at heterochromatic regions. To test this possibility, the incorporation of an inducible tagged version of histone $\mathrm{H} 3$ in a replication independent manner will be monitored as previously described [150]. This will allow us to determine whether acetylation of the H2B NTD contributes to the role of Clr3 in nucleosome positioning or nucleosome turnover.

We also plan to examine whether mutation of these sites of $\mathrm{H} 2 \mathrm{~B}$ acetylation lead to increased drug sensitivity. Since apoptotic-like death in S. cerevisiae is dependent on the ability of H2B S10 to be phosphorylated and this phosphorylation can be blocked by $\mathrm{H} 2 \mathrm{~B} \mathrm{~K} 11$ acetylation when cells are exposed to $\mathrm{H}_{2} \mathrm{O}_{2}$, it is possible this pathway is conserved in fission yeast. This will require a more in depth investigation of the corresponding acetylation site in fission yeast, H2B K10, which we currently do not have as a sole mutant. If this pathway is conserved in fission yeast, it is possible it could be complicating our analysis of heterochromatic silencing and must to be evaluated. In mice, 
$\mathrm{H} 2 \mathrm{~B}$ is also phosphorylated on $\mathrm{S} 14$ at sites of dsDNA breaks and this occurs in a $\gamma \mathrm{H} 2 \mathrm{~A}$ dependent manner [246]. Although this residue is not conserved in fission yeast, it is possible that $\mathrm{H} 2 \mathrm{~B}$ could have a role in DNA damage repair in which acetylation of $\mathrm{H} 2 \mathrm{~B}$ $\mathrm{K} 5,6,10$, and 15 could be important. We can test this by growing the $\mathrm{H} 2 \mathrm{~B}$ acetyl mimic strains on drugs which cause dsDNA breaks such as methyl methanesulfonate, a DNAalkylating agent, or bleomycin, which is a radiomimetic compound. This would allow us to further characterize the importance of these acetylation sites and their biological significance.

We believe these studies will be important due to the conservation of sites of acetylation between fission yeast and higher eukaryotes. The study of these processes in a simple model organism such as yeast will allow us to understand their implications in higher eukaryotes, where the pathways regulating events linked to the $\mathrm{H} 2 \mathrm{~B} \mathrm{~N}$-terminus are more complicated. It will also contribute to our knowledge of the H2B N-terminal tail, which has been far less characterized than that of histones $\mathrm{H} 3$ and $\mathrm{H} 4$.

\section{Mutations of DDX3X in Medulloblastoma}

Medulloblastoma affects many children worldwide and current treatments are general in nature rather than based on a particular medulloblastoma subtype. With the definition of medulloblastoma subtypes, more emphasis has been concentrated on developing specific treatments to target each subtype. Recent studies have begun to identify specific mutations and translocations associated with each subtype. Mutations of DDX3X are frequently found in WNT subtype tumors, leading to much research into the mechanism by which they aid in the development of medulloblastoma. This work identified that specific mutations of DDX3X (A222P, G302V, G325E, and P568L) are likely defective in the translational control of transcripts with long unstructured UTRs. This work has facilitated a better understanding of the biological defects associated with DDX3X mutations in hopes of aiding the development of more specific treatment regimens.

\section{Consequences of DDX3X Mutation In Vitro}

In conjunction with our examination of the biological defects associated with DDX3X mutants, the structure and helicase activity of the DDX3X was examined. Although the structure of the DDX3X core of D1 and D2 was previously solved, the protein did not exhibit the characteristic RNA-stimulated ATPase activity needed to study the effects of mutations in DDX3X [157, 247]. In collaboration with our work, a new construct was generated containing an N-terminal ABL, similar to that required by the D. melanogaster Vasa DEAD-box helicase for RNA-stimulated ATPase activity, along with the D1/D2 core. Once isolated using bacterial expression, this DDX3X construct exhibited RNA-stimulated ATPase activity, indicating the requirement for the ABL in RNA-stimulated ATP hydrolysis. A structure of the DDX3X core with the ABL was generated in the $\mathrm{D} 354 \mathrm{~V}$ background and suggests that this region can interact with 
ATP, similar to Vasa. NMR chemical shift perturbation (NMR CSP) was used to confirm the interaction of the ABL with ATP as suggested by the new structure [157].

We now have a better understanding of the physical consequences of several of the DDX3X mutations through a structural study of DDX3X. When the ATPase activity of DDX3X G302V, G325E, L353F, and D354V mutants which purified at good yields was measured, the DDX3X D354V mutant exhibited normal levels of ATPase activity in the presence of the preferred dsRNA/ssRNA hybrid substrate. In contrast the G302V and G325E mutants exhibited a significant decrease in ATPase activity, while the L353F mutant exhibited only a minor decrease. Both G302V and G325E mutations are located in motifs within D1 which interact with RNA. Using NMR CSP of D1, it was shown that residues G302 and G325 have significant shifts in the presence of the dsRNA substrate when monitored by residue specific CSP, confirming their importance in binding to dsRNA but the portion of D1 which binds to ssRNA could not be identified. Although they are both important in interacting with the RNA, only the G302V mutation was shown to be defective in dsRNA binding by EMSA. In contrast, G325E shows no defect in dsRNA binding, indicating it likely plays a larger role in the RNA-stimulated ATPase activity of DDX3X. While A222P and P568L were not able to be tested in these assays, their location in DDX3X provides some insight into the biological implications of mutations at these positions. A222P lies just upstream of the Walker A motif and may affect ATP binding and hydrolysis while P568L lies at the end of an alpha helix in D2 and may alter the structure of D2 by allowing the extension of an alpha helix beyond its normal boundary [156]. Further research will be required to determine the impact of DDX3X mutants currently unable to be purified.

\section{DDX3X Has a Conserved Role in Translation}

The defects in ATP hydrolysis and RNA binding align well with the fission yeast data which shows that DDX3X G302V and G325E mutants cannot complement for the ded1-1D5 temperature sensitive defect in translation. Although the involvement of DEAD-box helicases in translation can be traced back to the role of eIF4A in unwinding 5' secondary structure of mRNA, allowing for efficient ribosome binding, it wasn't until the late 1990's that Ded1p was shown to be involved in translation in vitro [192]. A similar phenomenon was observed in fission yeast where Ded1 was shown to be required for the general translation of RNAs, but had the strongest requirement for the translation of RNAs with long structured UTRs such as the B-type cyclins Cdc13 and Cig2. Shortening the 5'UTRs reduced the requirement of Ded1 for their translation [162]. In fission yeast the average 5'UTR is approximately $0.3 \mathrm{~kb}$, while the 5'UTRs of Cig2 and Cdc13 are longer than average at $1.4 \mathrm{~kb}$ and $0.7 \mathrm{~kb}$ respectively [248-250]. The UTRs of both genes are predicted to have a high amount of secondary structure. In our assay, the A222P, G302V, G325E, and P568L mutants failed to overcome the defect in Cig2 translation in contrast to WT DDX3X, which showed normal Cig2 protein levels.

Since DDX3X is able to rescue the ded1-1D5 and ded1-61 thermosensitive translation defects, it is likely that it serves a conserved function in translation of human 
mRNAs. DDX3X has been shown to interact with the nuclear mRNA export machinery and several eukaryotic translation initiation factors in mammalian cells. Studies in HeLa cells indicate that while DDX3X has little effect on general translation, it aids in the translation of genes with complex or long GC rich 5'UTRs such as the 5'UTR for TGF $\beta 1$, which has a highly stable stem-loop structure in its 5'UTR [175]. Knockdown of DDX3X in HeLa cells causes decreased cell growth, with delayed transition between G1 and $\mathrm{S}$ phase of the cell cycle. A screen for mRNAs that accumulate in translation initiation complexes due to difficulty in translation was performed in a DDX3X knockdown and mRNAs were identified as potential DDX3X targets. Cyclin E1, which is necessary for the G1/S transition and contains a highly GC rich 5'UTR, was identified from this screen. Translation of cyclin E1 was shown to be partially dependent on DDX3X helicase activity on the complex 5'UTR. Exchange of the cyclin E1 5'UTR with that of the less complex cyclin D1 5'UTRd eliminated the dependence on DDX3X for proper translation [174]. DDX3X is also important in the translation of HIV-1 viral mRNA. The 5' UTR of HIV-1 mRNA is highly complex, containing a TAR helix at the extreme 5' UTR along with a high degree of secondary structure throughout the UTR. It is suggested that DDX3X is needed to help relieve these structures and allow for proper ribosome binding to facilitate translation $[177,193]$. These results highlight the importance of DDX3X and its homologs in the translation of RNAs containing complex 5'UTRs, and that this function is likely conserved from fission yeast to humans.

\section{DDX3X Targets 5'UTRs and Coding Regions in Humans}

In normal cerebellar tissue, DDX3X expression is low with no distinct pattern of localization. In contrast, the G302V and G325E mutants assembled into stress granules at a higher rate than WT DDX3X. Stress granules indicate defects in translation initiation and consist of mRNAs and proteins involved in translation initiation [181, 251]. DDX3X High-throughput sequencing of RNA isolated by crosslinking immunoprecipitation (CLIP-Seq) and individual-nucleotide crosslinking and immunoprecipitation (FASTiCLIP) examining DDX3X showed that it localizes to both the UTRs and coding regions of mRNA from more than 11,000 genes. DDX3X enrichment in the 5'UTR is centered on the translation initiation codon $[180,181]$. No specific DDX3X binding site was uncovered, but DDX3X binds preferentially to GC rich regions, corresponding with previous findings suggesting DDX3X is necessary to resolve complex GC rich 5'UTRs [180]. Mutation of DDX3X led to ribosome accumulation within the 5' UTR along with a misregulation of translation, although mutant DDX3X can still interact with components of the translation initiation machinery $[180,181]$. Although the role of DDX3X in translation within human cells appears to be more of a global role than in fission yeast, the enrichment of DDX3X at 5'UTRs and GC rich regions suggest there is a conserved role in resolving complex 5'UTRs. We can also not discard a role for DDX3X in regions of mRNA other than the 5'UTR since our study did not address this. It is possible that DDX3X is also binding within the coding region of genes in fission yeast similar to its actions in humans cells, but this localization along with any impact on translation have yet to be elucidated. 


\section{Role of Translation in Cancer}

Much research has examined how alterations in translation contribute to various diseases. Altered transcriptional regulation has a role in the development and progression of numerous cancer types, often due to altered expression or activity of translation factors or factors regulating translation. One of the most studied examples of misregulation of translation involves changes in the levels of expression of members of the eIF4F complex, which plays a regulatory role in cap-dependent mRNA translation [252, 253]. This complex consists of eIF4E which binds the 5' mRNA cap, the scaffold protein eIF4G, and the DEAD-box RNA helicase eIF4A [254].

eIF4F plays a role in recruiting the $40 \mathrm{~S}$ ribosomal subunit and can resolve secondary structure of complex 5' UTRs allowing for efficient ribosome scanning and translation $[253,254]$. The least abundant member of this complex, eIF4E, plays a crucial role in regulating cap-dependent mRNA translation and is regulated by phosphorylation and inhibitory proteins. Increases in expression and changes in the phosphorylation levels of eIF4E along with altered expression of inhibitory proteins have been linked to many types of cancer and tend to indicate a poor prognosis [252,253, 255]. This can lead to increased translation of particular mRNAs with complex 5' UTRs, including many proteins involved in normal cell cycle progression. Overexpression of these proteins leads to aberrant cell cycle progression and aids in cancer development [252, 253, 256]. Interestingly, DDX3X has been shown to interact with eIF4E and eIF4G of the eIF4F complex [176, 257]. This link provides an interesting connection between DDX3X and its possible role in tumorigenesis.

\section{Possible Connection between DDX3X and Cancer}

Due to the link between DDX3X and eIF4E, it is likely that DDX3X has a crucial role in translation. However, reports on the function of this interaction vary. It has been reported that DDX3X inhibits eIF4E, but these authors also suggest DDX3X plays an inhibitory role towards global transcription [257]. This is contradictory to more recent reports suggesting DDX3X promotes translation and may be attributed to DDX3X overexpression leading to translational repression. Other report suggests DDX3X can interact with eIF4G and acts to promote the translation of particular mRNAs with complex 5' UTRs, such as mRNA of cyclin E1 and HIV-1 [174, 177]. Further research is needed to fully resolve these discrepancies.

Although the exact role in translation is unknown, it is easy to hypothesize that DDX3X aids eIF4A in the removal of complex structures from 5' UTRs. Mutations impairing RNA binding of DDX3X may lead to decreased translation and aid in transformation and tumorigenesis. It is also possible that other mutants may stabilize DDX3X or result in greater enzymatic activity which could also be oncogenic, as increased DDX3X levels have also been linked to cancer [178, 258]. Other work has described tumor suppressor like roles for DDX3X where it is involved in the upregulation of expression of $\mathrm{p} 21 \mathrm{waf} 1 / \mathrm{cip} 1, \mathrm{a} \mathrm{G}_{1} / \mathrm{S}$ cell cycle regulator, but only ATPase activity of 
DDX $3 \mathrm{X}$ is required for this regulation $[172,259]$. This suggests DDX $3 \mathrm{X}$ can also act independently of its helicase activity to regulate the cell cycle. Further studies will be necessary to fully resolve the role of DDX3X mutants in medulloblastoma, but the current research points towards the importance of altered translation being a key factor.

\section{Future Directions}

Although new evidence indicates that mutants of DDX3X found in medulloblastoma show translational defects, the mechanism behind this is not well understood. There is still much work to be done before we fully understand if DDX3X will be a useful target in treating medulloblastoma as new DDX3X inhibitors are being studied [260-262]. DDX3X in medulloblastoma has also been found to be altered in other cancers [263-267]. Although it has been shown that DDX3X binds to both 5' UTRs and coding regions, no distinct pattern has been observed. The role of DDX3X and DDX3X mutants in resolving complex UTRs is still unclear, with further experimentation needed to examine any contributions to cancer. Since transcriptional regulation may be intact in DDX3X mutants, it will also be necessary to look at translational profiles to understand the specific translational effect of DDX3X mutation. Simple organisms such as fission yeast, where the translational role of DDX3X appears conserved with Ded1, may be useful in determining if specific proteins, such as Cig2 are affected by mutation of DDX3X. If DDX $3 \mathrm{X}$ is playing a key role in transformation and tumorigenesis as current research suggests, it could provide insight into the development of new, more targeted treatment approaches in the future. 


\section{LIST OF REFERENCES}

1. Luger, K., A.W. Mader, R.K. Richmond, D.F. Sargent, and T.J. Richmond, Crystal structure of the nucleosome core particle at $2.8 \mathrm{~A}$ resolution. Nature, 1997. 389(6648): p. 251-60.

2. Burgess, R.J. and Z. Zhang, Histone chaperones in nucleosome assembly and human disease. Nat Struct Mol Biol, 2013. 20(1): p. 14-22.

3. Finch, J.T., M. Noll, and R.D. Kornberg, Electron microscopy of defined lengths of chromatin. Proc Natl Acad Sci U S A, 1975. 72(9): p. 3320-2.

4. Phillips, D.M., The presence of acetyl groups of histones. Biochem J, 1963. 87: p. 258-63.

5. Allfrey, V.G., R. Faulkner, and A.E. Mirsky, Acetylation and Methylation of Histones and their Possible Role in the Regulation of RNA Synthesis. Proc Natl Acad Sci U S A, 1964. 51: p. 786-94.

6. Kouzarides, T., Chromatin modifications and their function. Cell, 2007. 128(4): p. 693-705.

7. Grunstein, M., Histone acetylation in chromatin structure and transcription. Nature, 1997. 389(6649): p. 349-52.

8. Tamkun, J.W., R. Deuring, M.P. Scott, M. Kissinger, A.M. Pattatucci, T.C. Kaufman, and J.A. Kennison, brahma: a regulator of Drosophila homeotic genes structurally related to the yeast transcriptional activator SNF2/SWI2. Cell, 1992. 68(3): p. 561-72.

9. Rea, S., F. Eisenhaber, D. O'Carroll, B.D. Strahl, Z.W. Sun, M. Schmid, S. Opravil, K. Mechtler, C.P. Ponting, C.D. Allis, and T. Jenuwein, Regulation of chromatin structure by site-specific histone H3 methyltransferases. Nature, 2000. 406(6796): p. 593-9.

10. Martin, C. and Y. Zhang, The diverse functions of histone lysine methylation. Nat Rev Mol Cell Biol, 2005. 6(11): p. 838-49.

11. Dillon, S.C., X. Zhang, R.C. Trievel, and X. Cheng, The SET-domain protein superfamily: protein lysine methyltransferases. Genome Biol, 2005. 6(8): p. 227.

12. Stassen, M.J., D. Bailey, S. Nelson, V. Chinwalla, and P.J. Harte, The Drosophila trithorax proteins contain a novel variant of the nuclear receptor type DNA binding domain and an ancient conserved motif found in other chromosomal proteins. Mech Dev, 1995. 52(2-3): p. 209-23.

13. Passarge, E., Emil Heitz and the concept of heterochromatin: longitudinal chromosome differentiation was recognized fifty years ago. Am J Hum Genet, 1979. 31(2): p. 106-15.

14. Grewal, S.I. and S. Jia, Heterochromatin revisited. Nat Rev Genet, 2007. 8(1): p. $35-46$.

15. Woolcock, K.J. and M. Buhler, Nuclear organisation and RNAi in fission yeast. Curr Opin Cell Biol, 2013. 25(3): p. 372-7.

16. Trojer, P. and D. Reinberg, Facultative heterochromatin: is there a distinctive molecular signature? Mol Cell, 2007. 28(1): p. 1-13.

17. Gross, D.S., Heterochromatin: dark matter or variation on a theme? Curr Biol, 2015. 25(11): p. R462-5. 
18. Wutz, A., Gene silencing in X-chromosome inactivation: advances in understanding facultative heterochromatin formation. Nat Rev Genet, 2011. 12(8): p. 542-53.

19. Oberdoerffer, P. and D.A. Sinclair, The role of nuclear architecture in genomic instability and ageing. Nat Rev Mol Cell Biol, 2007. 8(9): p. 692-702.

20. Allshire, R.C., J.P. Javerzat, N.J. Redhead, and G. Cranston, Position effect variegation at fission yeast centromeres. Cell, 1994. 76(1): p. 157-69.

21. Allshire, R.C., E.R. Nimmo, K. Ekwall, J.P. Javerzat, and G. Cranston, Mutations derepressing silent centromeric domains in fission yeast disrupt chromosome segregation. Genes Dev, 1995. 9(2): p. 218-33.

22. Allshire, R.C. and K. Ekwall, Epigenetic Regulation of Chromatin States in Schizosaccharomyces pombe. Cold Spring Harb Perspect Biol, 2015. 7(7): p. 125.

23. Cam, H.P. and S. Whitehall, Analysis of Heterochromatin in Schizosaccharomyces pombe. Cold Spring Harb Protoc, 2016. 2016(11): p. 920927.

24. Elgin, S.C. and G. Reuter, Position-effect variegation, heterochromatin formation, and gene silencing in Drosophila. Cold Spring Harb Perspect Biol, 2013. 5(8): p. 1-26.

25. Wood, V., R. Gwilliam, M.A. Rajandream, M. Lyne, R. Lyne, A. Stewart, J. Sgouros, N. Peat, J. Hayles, S. Baker, D. Basham, S. Bowman, K. Brooks, D. Brown, S. Brown, T. Chillingworth, C. Churcher, M. Collins, R. Connor, A. Cronin, P. Davis, T. Feltwell, A. Fraser, S. Gentles, A. Goble, N. Hamlin, D. Harris, J. Hidalgo, G. Hodgson, S. Holroyd, T. Hornsby, S. Howarth, E.J. Huckle, S. Hunt, K. Jagels, K. James, L. Jones, M. Jones, S. Leather, S. McDonald, J. McLean, P. Mooney, S. Moule, K. Mungall, L. Murphy, D. Niblett, C. Odell, K. Oliver, S. O'Neil, D. Pearson, M.A. Quail, E. Rabbinowitsch, K. Rutherford, S. Rutter, D. Saunders, K. Seeger, S. Sharp, J. Skelton, M. Simmonds, R. Squares, S. Squares, K. Stevens, K. Taylor, R.G. Taylor, A. Tivey, S. Walsh, T. Warren, S. Whitehead, J. Woodward, G. Volckaert, R. Aert, J. Robben, B. Grymonprez, I. Weltjens, E. Vanstreels, M. Rieger, M. Schafer, S. Muller-Auer, C. Gabel, M. Fuchs, A. Dusterhoft, C. Fritzc, E. Holzer, D. Moestl, H. Hilbert, K. Borzym, I. Langer, A. Beck, H. Lehrach, R. Reinhardt, T.M. Pohl, P. Eger, W. Zimmermann, H. Wedler, R. Wambutt, B. Purnelle, A. Goffeau, E. Cadieu, S. Dreano, S. Gloux, V. Lelaure, S. Mottier, F. Galibert, S.J. Aves, Z. Xiang, C. Hunt, K. Moore, S.M. Hurst, M. Lucas, M. Rochet, C. Gaillardin, V.A. Tallada, A. Garzon, G. Thode, R.R. Daga, L. Cruzado, J. Jimenez, M. Sanchez, F. del Rey, J. Benito, A.

Dominguez, J.L. Revuelta, S. Moreno, J. Armstrong, S.L. Forsburg, L. Cerutti, T. Lowe, W.R. McCombie, I. Paulsen, J. Potashkin, G.V. Shpakovski, D. Ussery, B.G. Barrell and P. Nurse, The genome sequence of Schizosaccharomyces pombe. Nature, 2002. 415(6874): p. 871-80.

26. Hoffman, C.S., V. Wood, and P.A. Fantes, An Ancient Yeast for Young Geneticists: A Primer on the Schizosaccharomyces pombe Model System. Genetics, 2015. 201(2): p. 403-23.

27. Forsburg, S.L., Overview of Schizosaccharomyces pombe. Curr Protoc Mol Biol, 2003. 64(13): p. 1-13. 
28. Jacqueline Hayles, P.N., Introduction to Fission Yeast as a Model System. Fission Yeast: A Laboratory Manual, 2016 ed. A.M.C. Lain Hagan, Paul Nurse. 2016, Cold Spring Harbor, New York: Cold Spring Harbor Laboratory Press. p. 1-19.

29. Kelly, M., J. Burke, M. Smith, A. Klar, and D. Beach, Four mating-type genes control sexual differentiation in the fission yeast. Embo j, 1988. 7(5): p. 1537-47.

30. Creamer, K.M. and J.F. Partridge, RITS-connecting transcription, RNA interference, and heterochromatin assembly in fission yeast. Wiley Interdiscip Rev RNA, 2011. 2(5): p. 632-46.

31. Alper, B.J., B.R. Lowe, and J.F. Partridge, Centromeric heterochromatin assembly in fission yeast--balancing transcription, RNA interference and chromatin modification. Chromosome Res, 2012. 20(5): p. 521-34.

32. Takahashi, K., E.S. Chen, and M. Yanagida, Requirement of Mis6 centromere connector for localizing a CENP-A-like protein in fission yeast. Science, 2000. 288(5474): p. 2215-9.

33. Thon, G. and J. Verhein-Hansen, Four chromo-domain proteins of Schizosaccharomyces pombe differentially repress transcription at various chromosomal locations. Genetics, 2000. 155(2): p. 551-68.

34. Compton, D.A., Mechanisms of aneuploidy. Curr Opin Cell Biol, 2011. 23(1): p. 109-13.

35. Ekwall, K. and T. Ruusala, Mutations in rik1, clr2, clr3 and clr4 genes asymmetrically derepress the silent mating-type loci in fission yeast. Genetics, 1994. 136(1): p. 53-64.

36. Ekwall, K., T. Olsson, B.M. Turner, G. Cranston, and R.C. Allshire, Transient inhibition of histone deacetylation alters the structural and functional imprint at fission yeast centromeres. Cell, 1997. 91(7): p. 1021-32.

37. Nimmo, E.R., G. Cranston, and R.C. Allshire, Telomere-associated chromosome breakage in fission yeast results in variegated expression of adjacent genes. Embo j, 1994. 13(16): p. 3801-11.

38. Pidoux, A.L. and R.C. Allshire, Kinetochore and heterochromatin domains of the fission yeast centromere. Chromosome Res, 2004. 12(6): p. 521-34.

39. Bernard, P., J.F. Maure, J.F. Partridge, S. Genier, J.P. Javerzat, and R.C. Allshire, Requirement of heterochromatin for cohesion at centromeres. Science, 2001. 294(5551): p. 2539-42.

40. Nonaka, N., T. Kitajima, S. Yokobayashi, G. Xiao, M. Yamamoto, S.I. Grewal, and Y. Watanabe, Recruitment of cohesin to heterochromatic regions by Swi6/HP1 in fission yeast. Nat Cell Biol, 2002. 4(1): p. 89-93.

41. Lorentz, A., K. Ostermann, O. Fleck, and H. Schmidt, Switching gene swi6, involved in repression of silent mating-type loci in fission yeast, encodes a homologue of chromatin-associated proteins from Drosophila and mammals. Gene, 1994. 143(1): p. 139-43.

42. Wiles, E.T. and E.U. Selker, H3K27 methylation: a promiscuous repressive chromatin mark. Curr Opin Genet Dev, 2016. 43: p. 31-37.

43. Trievel, R.C., B.M. Beach, L.M. Dirk, R.L. Houtz, and J.H. Hurley, Structure and catalytic mechanism of a SET domain protein methyltransferase. Cell, 2002. 111(1): p. 91-103. 
44. Min, J., X. Zhang, X. Cheng, S.I. Grewal, and R.M. Xu, Structure of the SET domain histone lysine methyltransferase Clr4. Nat Struct Biol, 2002. 9(11): p. 828-32.

45. Bannister, A.J., P. Zegerman, J.F. Partridge, E.A. Miska, J.O. Thomas, R.C. Allshire, and T. Kouzarides, Selective recognition of methylated lysine 9 on histone H3 by the HP1 chromo domain. Nature, 2001. 410(6824): p. 120-4.

46. Lachner, M., D. O'Carroll, S. Rea, K. Mechtler, and T. Jenuwein, Methylation of histone H3 lysine 9 creates a binding site for HP1 proteins. Nature, 2001. 410(6824): p. 116-20.

47. Fischer, T., B. Cui, J. Dhakshnamoorthy, M. Zhou, C. Rubin, M. Zofall, T.D. Veenstra, and S.I. Grewal, Diverse roles of HP1 proteins in heterochromatin assembly and functions in fission yeast. Proc Natl Acad Sci U S A, 2009. 106(22): p. 8998-9003.

48. Motamedi, M.R., E.J. Hong, X. Li, S. Gerber, C. Denison, S. Gygi, and D. Moazed, HP1 proteins form distinct complexes and mediate heterochromatic gene silencing by nonoverlapping mechanisms. Mol Cell, 2008. 32(6): p. 778-90.

49. Thon, G., K.R. Hansen, S.P. Altes, D. Sidhu, G. Singh, J. Verhein-Hansen, M.J. Bonaduce, and A.J. Klar, The Clr7 and Clr8 directionality factors and the Pcu4 cullin mediate heterochromatin formation in the fission yeast Schizosaccharomyces pombe. Genetics, 2005. 171(4): p. 1583-95.

50. Horn, P.J., J.N. Bastie, and C.L. Peterson, A Rikl-associated, cullin-dependent E3 ubiquitin ligase is essential for heterochromatin formation. Genes Dev, 2005. 19(14): p. 1705-14.

51. Jia, S., R. Kobayashi, and S.I. Grewal, Ubiquitin ligase component Cul4 associates with Clr4 histone methyltransferase to assemble heterochromatin. Nat Cell Biol, 2005. 7(10): p. 1007-13.

52. Bayne, E.H., S.A. White, A. Kagansky, D.A. Bijos, L. Sanchez-Pulido, K.L. Hoe, D.U. Kim, H.O. Park, C.P. Ponting, J. Rappsilber, and R.C. Allshire, Stc1: a critical link between RNAi and chromatin modification required for heterochromatin integrity. Cell, 2010. 140(5): p. 666-77.

53. Li, F., M. Huarte, M. Zaratiegui, M.W. Vaughn, Y. Shi, R. Martienssen, and W.Z. Cande, Lid2 is required for coordinating $\mathrm{H} 3 \mathrm{~K} 4$ and $\mathrm{H} 3 \mathrm{~K} 9$ methylation of heterochromatin and euchromatin. Cell, 2008. 135(2): p. 272-83.

54. Goto, D.B. and J. Nakayama, RNA and epigenetic silencing: insight from fission yeast. Dev Growth Differ, 2012. 54(1): p. 129-41.

55. Zhang, K., K. Mosch, W. Fischle, and S.I. Grewal, Roles of the Clr4 methyltransferase complex in nucleation, spreading and maintenance of heterochromatin. Nat Struct Mol Biol, 2008. 15(4): p. 381-8.

56. Volpe, T.A., C. Kidner, I.M. Hall, G. Teng, S.I. Grewal, and R.A. Martienssen, Regulation of heterochromatic silencing and histone H3 lysine-9 methylation by RNAi. Science, 2002. 297(5588): p. 1833-7.

57. Kato, H., D.B. Goto, R.A. Martienssen, T. Urano, K. Furukawa, and Y. Murakami, RNA polymerase II is required for RNAi-dependent heterochromatin assembly. Science, 2005. 309(5733): p. 467-9.

58. Lejeune, E., M. Bortfeld, S.A. White, A.L. Pidoux, K. Ekwall, R.C. Allshire, and A.G. Ladurner, The chromatin-remodeling factor FACT contributes to 
centromeric heterochromatin independently of RNAi. Curr Biol, 2007. 17(14): p. 1219-24.

59. Kiely, C.M., S. Marguerat, J.F. Garcia, H.D. Madhani, J. Bahler, and F. Winston, Spt6 is required for heterochromatic silencing in the fission yeast

Schizosaccharomyces pombe. Mol Cell Biol, 2011. 31(20): p. 4193-204.

60. Chen, E.S., K. Zhang, E. Nicolas, H.P. Cam, M. Zofall, and S.I. Grewal, Cell cycle control of centromeric repeat transcription and heterochromatin assembly. Nature, 2008. 451(7179): p. 734-7.

61. Kloc, A., M. Zaratiegui, E. Nora, and R. Martienssen, RNA interference guides histone modification during the $S$ phase of chromosomal replication. Curr Biol, 2008. 18(7): p. 490-5.

62. Smialowska, A., I. Djupedal, J. Wang, P. Kylsten, P. Swoboda, and K. Ekwall, RNAi mediates post-transcriptional repression of gene expression in fission yeast Schizosaccharomyces pombe. Biochem Biophys Res Commun, 2014. 444(2): p. 254-9.

63. Motamedi, M.R., A. Verdel, S.U. Colmenares, S.A. Gerber, S.P. Gygi, and D. Moazed, Two RNAi complexes, RITS and RDRC, physically interact and localize to noncoding centromeric RNAs. Cell, 2004. 119(6): p. 789-802.

64. Sugiyama, T., H. Cam, A. Verdel, D. Moazed, and S.I. Grewal, RNA-dependent $R N A$ polymerase is an essential component of a self-enforcing loop coupling heterochromatin assembly to siRNA production. Proc Natl Acad Sci U S A, 2005. 102(1): p. 152-7.

65. Buker, S.M., T. Iida, M. Buhler, J. Villen, S.P. Gygi, J. Nakayama, and D. Moazed, Two different Argonaute complexes are required for siRNA generation and heterochromatin assembly in fission yeast. Nat Struct Mol Biol, 2007. 14(3): p. 200-7.

66. Irvine, D.V., M. Zaratiegui, N.H. Tolia, D.B. Goto, D.H. Chitwood, M.W. Vaughn, L. Joshua-Tor, and R.A. Martienssen, Argonaute slicing is required for heterochromatic silencing and spreading. Science, 2006. 313(5790): p. 1134-7.

67. Verdel, A., S. Jia, S. Gerber, T. Sugiyama, S. Gygi, S.I. Grewal, and D. Moazed, RNAi-mediated targeting of heterochromatin by the RITS complex. Science, 2004. 303(5658): p. 672-6.

68. Schalch, T., G. Job, S. Shanker, J.F. Partridge, and L. Joshua-Tor, The Chp1-Tas3 core is a multifunctional platform critical for gene silencing by RITS. Nat Struct Mol Biol, 2011. 18(12): p. 1351-7.

69. Partridge, J.F., K.S. Scott, A.J. Bannister, T. Kouzarides, and R.C. Allshire, cisacting DNA from fission yeast centromeres mediates histone H3 methylation and recruitment of silencing factors and cohesin to an ectopic site. Curr Biol, 2002. 12(19): p. 1652-60.

70. He, C., S.S. Pillai, F. Taglini, F. Li, K. Ruan, J. Zhang, J. Wu, Y. Shi, and E.H. Bayne, Structural analysis of Stcl provides insights into the coupling of RNAi and chromatin modification. Proc Natl Acad Sci U S A, 2013. 110(21): p. E1879-88.

71. Lejeune, E., E.H. Bayne, and R.C. Allshire, On the connection between RNAi and heterochromatin at centromeres. Cold Spring Harb Symp Quant Biol, 2010. 75: p. 275-83. 
72. Saksouk, N., E. Simboeck, and J. Dejardin, Constitutive heterochromatin formation and transcription in mammals. Epigenetics Chromatin, 2015. 8: p. 3.

73. Buhler, M. and D. Moazed, Transcription and RNAi in heterochromatic gene silencing. Nat Struct Mol Biol, 2007. 14(11): p. 1041-8.

74. Fukagawa, T., M. Nogami, M. Yoshikawa, M. Ikeno, T. Okazaki, Y. Takami, T. Nakayama, and M. Oshimura, Dicer is essential for formation of the heterochromatin structure in vertebrate cells. Nat Cell Biol, 2004. 6(8): p. 78491.

75. Rudert, F., S. Bronner, J.M. Garnier, and P. Dolle, Transcripts from opposite strands of gamma satellite DNA are differentially expressed during mouse development. Mamm Genome, 1995. 6(2): p. 76-83.

76. Lu, J. and D.M. Gilbert, Proliferation-dependent and cell cycle regulated transcription of mouse pericentric heterochromatin. J Cell Biol, 2007. 179(3): p. 411-21.

77. Santenard, A., C. Ziegler-Birling, M. Koch, L. Tora, A.J. Bannister, and M.E. Torres-Padilla, Heterochromatin formation in the mouse embryo requires critical residues of the histone variant H3.3. Nat Cell Biol, 2010. 12(9): p. 853-62.

78. Sadaie, M., T. Iida, T. Urano, and J. Nakayama, A chromodomain protein, Chp1, is required for the establishment of heterochromatin in fission yeast. Embo $\mathrm{j}$, 2004. 23(19): p. 3825-35.

79. Shanker, S., G. Job, O.L. George, K.M. Creamer, A. Shaban, and J.F. Partridge, Continuous requirement for the Clr4 complex but not RNAi for centromeric heterochromatin assembly in fission yeast harboring a disrupted RITS complex. PLoS Genet, 2010. 6(10): p. e1001174.

80. Hall, I.M., G.D. Shankaranarayana, K. Noma, N. Ayoub, A. Cohen, and S.I. Grewal, Establishment and maintenance of a heterochromatin domain. Science, 2002. 297(5590): p. 2232-7.

81. Baum, M., V.K. Ngan, and L. Clarke, The centromeric K-type repeat and the central core are together sufficient to establish a functional Schizosaccharomyces pombe centromere. Mol Biol Cell, 1994. 5(7): p. 747-61.

82. Buscaino, A., E. Lejeune, P. Audergon, G. Hamilton, A. Pidoux, and R.C. Allshire, Distinct roles for Sir2 and RNAi in centromeric heterochromatin nucleation, spreading and maintenance. Embo j, 2013. 32(9): p. 1250-64.

83. Alper, B.J., G. Job, R.K. Yadav, S. Shanker, B.R. Lowe, and J.F. Partridge, Sir2 is required for Clr4 to initiate centromeric heterochromatin assembly in fission yeast. Embo j, 2013. 32(17): p. 2321-35.

84. Partridge, J.F., J.L. DeBeauchamp, A.M. Kosinski, D.L. Ulrich, M.J. Hadler, and V.J. Noffsinger, Functional separation of the requirements for establishment and maintenance of centromeric heterochromatin. Mol Cell, 2007. 26(4): p. 593-602.

85. Schalch, T., G. Job, V.J. Noffsinger, S. Shanker, C. Kuscu, L. Joshua-Tor, and J.F. Partridge, High-affinity binding of Chpl chromodomain to K9 methylated histone H3 is required to establish centromeric heterochromatin. Mol Cell, 2009. 34(1): p. 36-46.

86. Jia, S., K. Noma, and S.I. Grewal, RNAi-independent heterochromatin nucleation by the stress-activated ATF/CREB family proteins. Science, 2004. 304(5679): $\mathrm{p}$. 1971-6. 
87. Noma, K., C.D. Allis, and S.I. Grewal, Transitions in distinct histone H3 methylation patterns at the heterochromatin domain boundaries. Science, 2001. 293(5532): p. 1150-5.

88. Kim, H.S., E.S. Choi, J.A. Shin, Y.K. Jang, and S.D. Park, Regulation of Swi6/HP1-dependent heterochromatin assembly by cooperation of components of the mitogen-activated protein kinase pathway and a histone deacetylase Clr6. J Biol Chem, 2004. 279(41): p. 42850-9.

89. Yamada, T., W. Fischle, T. Sugiyama, C.D. Allis, and S.I. Grewal, The nucleation and maintenance of heterochromatin by a histone deacetylase in fission yeast. Mol Cell, 2005. 20(2): p. 173-85.

90. Grewal, S.I., M.J. Bonaduce, and A.J. Klar, Histone deacetylase homologs regulate epigenetic inheritance of transcriptional silencing and chromosome segregation in fission yeast. Genetics, 1998. 150(2): p. 563-76.

91. Cooper, J.P., E.R. Nimmo, R.C. Allshire, and T.R. Cech, Regulation of telomere length and function by a Myb-domain protein in fission yeast. Nature, 1997. 385(6618): p. 744-7.

92. Kanoh, J., M. Sadaie, T. Urano, and F. Ishikawa, Telomere binding protein Taz1 establishes Swi6 heterochromatin independently of RNAi at telomeres. Curr Biol, 2005. 15(20): p. 1808-19.

93. Inoue, A. and D. Fujimoto, Enzymatic deacetylation of histone. Biochem Biophys Res Commun, 1969. 36(1): p. 146-50.

94. Gershey, E.L., G. Vidali, and V.G. Allfrey, Chemical studies of histone acetylation. The occurrence of epsilon-N-acetyllysine in the f2al histone. J Biol Chem, 1968. 243(19): p. 5018-22.

95. Seto, E. and M. Yoshida, Erasers of histone acetylation: the histone deacetylase enzymes. Cold Spring Harb Perspect Biol, 2014. 6(4): p. a018713.

96. de Ruijter, A.J., A.H. van Gennip, H.N. Caron, S. Kemp, and A.B. van Kuilenburg, Histone deacetylases (HDACs): characterization of the classical HDAC family. Biochem J, 2003. 370(3): p. 737-49.

97. Sinha, I., M. Wiren, and K. Ekwall, Genome-wide patterns of histone modifications in fission yeast. Chromosome Res, 2006. 14(1): p. 95-105.

98. Wang, A., S.K. Kurdistani, and M. Grunstein, Requirement of Hos 2 histone deacetylase for gene activity in yeast. Science, 2002. 298(5597): p. 1412-4.

99. Freeman-Cook, L.L., J.M. Sherman, C.B. Brachmann, R.C. Allshire, J.D. Boeke, and L. Pillus, The Schizosaccharomyces pombe hst4(+) gene is a SIR2 homologue with silencing and centromeric functions. Mol Biol Cell, 1999. 10(10): p. 317186.

100. Durand-Dubief, M., I. Sinha, F. Fagerstrom-Billai, C. Bonilla, A. Wright, M. Grunstein, and K. Ekwall, Specific functions for the fission yeast Sirtuins Hst 2 and Hst 4 in gene regulation and retrotransposon silencing. Embo j, 2007. 26(10): p. 2477-88.

101. Wiren, M., R.A. Silverstein, I. Sinha, J. Walfridsson, H.M. Lee, P. Laurenson, L. Pillus, D. Robyr, M. Grunstein, and K. Ekwall, Genomewide analysis of nucleosome density histone acetylation and HDAC function in fission yeast. Embo j, 2005. 24(16): p. 2906-18. 
102. Garcia, J.F., P.A. Dumesic, P.D. Hartley, H. El-Samad, and H.D. Madhani, Combinatorial, site-specific requirement for heterochromatic silencing factors in the elimination of nucleosome-free regions. Genes Dev, 2010. 24(16): p. 1758-71.

103. Smith, B.C., W.C. Hallows, and J.M. Denu, Mechanisms and molecular probes of sirtuins. Chem Biol, 2008. 15(10): p. 1002-13.

104. Freeman-Cook, L.L., E.B. Gomez, E.J. Spedale, J. Marlett, S.L. Forsburg, L. Pillus, and P. Laurenson, Conserved locus-specific silencing functions of Schizosaccharomyces pombe sir2+. Genetics, 2005. 169(3): p. 1243-60.

105. Haldar, D. and R.T. Kamakaka, Schizosaccharomyces pombe Hst4 functions in DNA damage response by regulating histone H3 K56 acetylation. Eukaryot Cell, 2008. 7(5): p. 800-13.

106. Costantini, S., A. Sharma, R. Raucci, M. Costantini, I. Autiero, and G. Colonna, Genealogy of an ancient protein family: the Sirtuins, a family of disordered members. BMC Evol Biol, 2013. 13(60): p. 1-19.

107. Greiss, S. and A. Gartner, Sirtuin/Sir2 phylogeny, evolutionary considerations and structural conservation. Mol Cells, 2009. 28(5): p. 407-15.

108. Sanders, B.D., B. Jackson, and R. Marmorstein, Structural basis for sirtuin function: what we know and what we don't. Biochim Biophys Acta, 2010. 1804(8): p. 1604-16.

109. Flick, F. and B. Luscher, Regulation of sirtuin function by posttranslational modifications. Front Pharmacol, 2012. 3(29): p. 1-13.

110. North, B.J. and E. Verdin, Sirtuins: Sir2-related NAD-dependent protein deacetylases. Genome Biol, 2004. 5(5): p. 224.

111. Kueng, S., M. Oppikofer, and S.M. Gasser, SIR proteins and the assembly of silent chromatin in budding yeast. Annu Rev Genet, 2013. 47: p. 275-306.

112. Grunstein, M. and S.M. Gasser, Epigenetics in Saccharomyces cerevisiae. Cold Spring Harb Perspect Biol, 2013. 5(7): p.1-28.

113. Vassilopoulos, A., K.S. Fritz, D.R. Petersen, and D. Gius, The human sirtuin family: evolutionary divergences and functions. Hum Genomics, 2011. 5(5): p. 485-96.

114. Chang, H.C. and L. Guarente, SIRT1 and other sirtuins in metabolism. Trends Endocrinol Metab, 2014. 25(3): p. 138-45.

115. Wilking, M.J. and N. Ahmad, The role of SIRT1 in cancer: the saga continues. Am J Pathol, 2015. 185(1): p. 26-8.

116. Muth, V., S. Nadaud, I. Grummt, and R. Voit, Acetylation of TAF(I)68, a subunit of TIF-IB/SL1, activates RNA polymerase I transcription. Embo j, 2001. 20(6): p. 1353-62.

117. Vaquero, A., R. Sternglanz, and D. Reinberg, NAD+-dependent deacetylation of H4 lysine 16 by class III HDACs. Oncogene, 2007. 26(37): p. 5505-20.

118. Vaquero, A., M. Scher, H. Erdjument-Bromage, P. Tempst, L. Serrano, and D. Reinberg, SIRT1 regulates the histone methyl-transferase SUV39H1 during heterochromatin formation. Nature, 2007. 450(7168): p. 440-4.

119. Shankaranarayana, G.D., M.R. Motamedi, D. Moazed, and S.I. Grewal, Sir2 regulates histone $\mathrm{H} 3$ lysine 9 methylation and heterochromatin assembly in fission yeast. Curr Biol, 2003. 13(14): p. 1240-6. 
120. Abshiru, N., R.E. Rajan, A. Verreault, and P. Thibault, Unraveling Site-Specific and Combinatorial Histone Modifications Using High-Resolution Mass Spectrometry in Histone Deacetylase Mutants of Fission Yeast. J Proteome Res, 2016. 15(7): p. 2132-42.

121. Imai, S., C.M. Armstrong, M. Kaeberlein, and L. Guarente, Transcriptional silencing and longevity protein Sir2 is an NAD-dependent histone deacetylase. Nature, 2000. 403(6771): p. 795-800.

122. Min, J., J. Landry, R. Sternglanz, and R.M. Xu, Crystal structure of a SIR2 homolog-NAD complex. Cell, 2001. 105(2): p. 269-79.

123. Sasaki, T., B. Maier, K.D. Koclega, M. Chruszcz, W. Gluba, P.T. Stukenberg, W. Minor, and H. Scrable, Phosphorylation regulates SIRT1 function. PLoS One, 2008. 3(12): p. e4020.

124. Ford, J., S. Ahmed, S. Allison, M. Jiang, and J. Milner, JNK2-dependent regulation of SIRT1 protein stability. Cell Cycle, 2008. 7(19): p. 3091-7.

125. Zschoernig, B. and U. Mahlknecht, Carboxy-terminal phosphorylation of SIRT1 by protein kinase $C K 2$. Biochem Biophys Res Commun, 2009. 381(3): p. 372-7.

126. Nasrin, N., V.K. Kaushik, E. Fortier, D. Wall, K.J. Pearson, R. de Cabo, and L. Bordone, JNK1 phosphorylates SIRT1 and promotes its enzymatic activity. PLoS One, 2009. 4(12): p. e8414.

127. Guo, X., J.G. Williams, T.T. Schug, and X. Li, DYRK1A and DYRK3 promote cell survival through phosphorylation and activation of SIRT1. J Biol Chem, 2010. 285(17): p. 13223-32.

128. Kang, H., J.W. Jung, M.K. Kim, and J.H. Chung, CK2 is the regulator of SIRT1 substrate-binding affinity, deacetylase activity and cellular response to DNAdamage. PLoS One, 2009. 4(8): p. e6611.

129. Yang, Y., W. Fu, J. Chen, N. Olashaw, X. Zhang, S.V. Nicosia, K. Bhalla, and W. Bai, SIRT1 sumoylation regulates its deacetylase activity and cellular response to genotoxic stress. Nat Cell Biol, 2007. 9(11): p. 1253-62.

130. Hannan, A., N.M. Abraham, S. Goyal, I. Jamir, U.D. Priyakumar, and K. Mishra, Sumoylation of Sir2 differentially regulates transcriptional silencing in yeast. Nucleic Acids Res, 2015. 43(21): p. 10213-26.

131. Clemente, S., L. Franco, and G. Lopez-Rodas, Distinct site specificity of two pea histone deacetylase complexes. Biochemistry, 2001. 40(35): p. 10671-6.

132. Hildmann, C., D. Wegener, D. Riester, R. Hempel, A. Schober, J. Merana, L. Giurato, S. Guccione, T.K. Nielsen, R. Ficner, and A. Schwienhorst, Substrate and inhibitor specificity of class 1 and class 2 histone deacetylases. J Biotechnol, 2006. 124(1): p. 258-70.

133. Riester, D., C. Hildmann, S. Grunewald, T. Beckers, and A. Schwienhorst, Factors affecting the substrate specificity of histone deacetylases. Biochem Biophys Res Commun, 2007. 357(2): p. 439-45.

134. Ekwall, K., E.R. Nimmo, J.P. Javerzat, B. Borgstrom, R. Egel, G. Cranston, and R. Allshire, Mutations in the fission yeast silencing factors clr4+ and rik1+ disrupt the localisation of the chromo domain protein Swi6p and impair centromere function. J Cell Sci, 1996. 109(11): p. 2637-48.

135. Bjerling, P., R.A. Silverstein, G. Thon, A. Caudy, S. Grewal, and K. Ekwall, Functional divergence between histone deacetylases in fission yeast by distinct 
cellular localization and in vivo specificity. Mol Cell Biol, 2002. 22(7): p. 217081.

136. Tong, J.K., C.A. Hassig, G.R. Schnitzler, R.E. Kingston, and S.L. Schreiber, Chromatin deacetylation by an ATP-dependent nucleosome remodelling complex. Nature, 1998. 395(6705): p. 917-21.

137. Wade, P.A., P.L. Jones, D. Vermaak, and A.P. Wolffe, A multiple subunit Mi-2 histone deacetylase from Xenopus laevis cofractionates with an associated Snf2 superfamily ATPase. Curr Biol, 1998. 8(14): p. 843-6.

138. Xue, Y., J. Wong, G.T. Moreno, M.K. Young, J. Cote, and W. Wang, NURD, a novel complex with both ATP-dependent chromatin-remodeling and histone deacetylase activities. Mol Cell, 1998. 2(6): p. 851-61.

139. Lai, A.Y. and P.A. Wade, Cancer biology and NuRD: a multifaceted chromatin remodelling complex. Nat Rev Cancer, 2011. 11(8): p. 588-96.

140. Allen, H.F., P.A. Wade, and T.G. Kutateladze, The NuRD architecture. Cell Mol Life Sci, 2013. 70(19): p. 3513-24.

141. Alqarni, S.S., A. Murthy, W. Zhang, M.R. Przewloka, A.P. Silva, A.A. Watson, S. Lejon, X.Y. Pei, A.H. Smits, S.L. Kloet, H. Wang, N.E. Shepherd, P.H. Stokes, G.A. Blobel, M. Vermeulen, D.M. Glover, J.P. Mackay, and E.D. Laue, Insight into the architecture of the NuRD complex: structure of the RbAp48-MTA1 subcomplex. J Biol Chem, 2014. 289(32): p. 21844-55.

142. Torchy, M.P., A. Hamiche, and B.P. Klaholz, Structure and function insights into the NuRD chromatin remodeling complex. Cell Mol Life Sci, 2015. 72(13): p. 2491-507.

143. Basta, J. and M. Rauchman, The nucleosome remodeling and deacetylase complex in development and disease. Transl Res, 2015. 165(1): p. 36-47.

144. Sugiyama, T., H.P. Cam, R. Sugiyama, K. Noma, M. Zofall, R. Kobayashi, and S.I. Grewal, SHREC, an effector complex for heterochromatic transcriptional silencing. Cell, 2007. 128(3): p. 491-504.

145. Creamer, K.M., G. Job, S. Shanker, G.A. Neale, Y.C. Lin, B. Bartholomew, and J.F. Partridge, The Mi-2 homolog Mit1 actively positions nucleosomes within heterochromatin to suppress transcription. Mol Cell Biol, 2014. 34(11): p. 204661.

146. Halverson, D., G. Gutkin, and L. Clarke, A novel member of the Swi6p family of fission yeast chromo domain-containing proteins associates with the centromere in vivo and affects chromosome segregation. Mol Gen Genet, 2000. 264(4): p. 492-505.

147. Partridge, J.F., B. Borgstrom, and R.C. Allshire, Distinct protein interaction domains and protein spreading in a complex centromere. Genes Dev, 2000. 14(7): p. 783-91.

148. Thon, G. and A.J. Klar, The clr1 locus regulates the expression of the cryptic mating-type loci of fission yeast. Genetics, 1992. 131(2): p. 287-96.

149. Job, G., C. Brugger, T. Xu, B.R. Lowe, Y. Pfister, C. Qu, S. Shanker, J.I. Banos Sanz, J.F. Partridge, and T. Schalch, SHREC Silences Heterochromatin via Distinct Remodeling and Deacetylation Modules. Mol Cell, 2016. 62(2): p. 20721. 
150. Aygun, O., S. Mehta, and S.I. Grewal, HDAC-mediated suppression of histone turnover promotes epigenetic stability of heterochromatin. Nat Struct Mol Biol, 2013. 20(5): p. 547-54.

151. Anderson, D.C., G.R. Green, K. Smith, and E.U. Selker, Extensive and varied modifications in histone $H 2 B$ of wild-type and histone deacetylase 1 mutant Neurospora crassa. Biochemistry, 2010. 49(25): p. 5244-57.

152. Xiong, L. and Y. Wang, Mapping Post-translational Modifications of Histones H2A, H2B and H4 in Schizosaccharomyces pombe. Int J Mass Spectrom, 2011. 301(1-3): p. 159-165.

153. Parra, M.A., D. Kerr, D. Fahy, D.J. Pouchnik, and J.J. Wyrick, Deciphering the roles of the histone $H 2 B N$-terminal domain in genome-wide transcription. Mol Cell Biol, 2006. 26(10): p. 3842-52.

154. Robinson, G., M. Parker, T.A. Kranenburg, C. Lu, X. Chen, L. Ding, T.N. Phoenix, E. Hedlund, L. Wei, X. Zhu, N. Chalhoub, S.J. Baker, R. Huether, R. Kriwacki, N. Curley, R. Thiruvenkatam, J. Wang, G. Wu, M. Rusch, X. Hong, J. Becksfort, P. Gupta, J. Ma, J. Easton, B. Vadodaria, A. Onar-Thomas, T. Lin, S. Li, S. Pounds, S. Paugh, D. Zhao, D. Kawauchi, M.F. Roussel, D. Finkelstein, D.W. Ellison, C.C. Lau, E. Bouffet, T. Hassall, S. Gururangan, R. Cohn, R.S. Fulton, L.L. Fulton, D.J. Dooling, K. Ochoa, A. Gajjar, E.R. Mardis, R.K. Wilson, J.R. Downing, J. Zhang, and R.J. Gilbertson, Novel mutations target distinct subgroups of medulloblastoma. Nature, 2012. 488(7409): p. 43-8.

155. Jones, D.T., N. Jager, M. Kool, T. Zichner, B. Hutter, M. Sultan, Y.J. Cho, T.J. Pugh, V. Hovestadt, A.M. Stutz, T. Rausch, H.J. Warnatz, M. Ryzhova, S. Bender, D. Sturm, S. Pleier, H. Cin, E. Pfaff, L. Sieber, A. Wittmann, M. Remke, H. Witt, S. Hutter, T. Tzaridis, J. Weischenfeldt, B. Raeder, M. Avci, V. Amstislavskiy, M. Zapatka, U.D. Weber, Q. Wang, B. Lasitschka, C.C. Bartholomae, M. Schmidt, C. von Kalle, V. Ast, C. Lawerenz, J. Eils, R. Kabbe, V. Benes, P. van Sluis, J. Koster, R. Volckmann, D. Shih, M.J. Betts, R.B. Russell, S. Coco, G.P. Tonini, U. Schuller, V. Hans, N. Graf, Y.J. Kim, C. Monoranu, W. Roggendorf, A. Unterberg, C. Herold-Mende, T. Milde, A.E. Kulozik, A. von Deimling, O. Witt, E. Maass, J. Rossler, M. Ebinger, M.U. Schuhmann, M.C. Fruhwald, M. Hasselblatt, N. Jabado, S. Rutkowski, A.O. von Bueren, D. Williamson, S.C. Clifford, M.G. McCabe, V.P. Collins, S. Wolf, S. Wiemann, H. Lehrach, B. Brors, W. Scheurlen, J. Felsberg, G. Reifenberger, P.A. Northcott, M.D. Taylor, M. Meyerson, S.L. Pomeroy, M.L. Yaspo, J.O. Korbel, A. Korshunov, R. Eils, S.M. Pfister, and P. Lichter, Dissecting the genomic complexity underlying medulloblastoma. Nature, 2012. 488(7409): p. 100-5.

156. Pugh, T.J., S.D. Weeraratne, T.C. Archer, D.A. Pomeranz Krummel, D. Auclair, J. Bochicchio, M.O. Carneiro, S.L. Carter, K. Cibulskis, R.L. Erlich, H. Greulich, M.S. Lawrence, N.J. Lennon, A. McKenna, J. Meldrim, A.H. Ramos, M.G. Ross, C. Russ, E. Shefler, A. Sivachenko, B. Sogoloff, P. Stojanov, P. Tamayo, J.P. Mesirov, V. Amani, N. Teider, S. Sengupta, J.P. Francois, P.A. Northcott, M.D. Taylor, F. Yu, G.R. Crabtree, A.G. Kautzman, S.B. Gabriel, G. Getz, N. Jager, D.T. Jones, P. Lichter, S.M. Pfister, T.M. Roberts, M. Meyerson, S.L. Pomeroy, and Y.J. Cho, Medulloblastoma exome sequencing uncovers subtype-specific somatic mutations. Nature, 2012. 488(7409): p. 106-10. 
157. Epling, L.B., C.R. Grace, B.R. Lowe, J.F. Partridge, and E.J. Enemark, Cancerassociated mutants of RNA helicase DDX3X are defective in RNA-stimulated ATP hydrolysis. J Mol Biol, 2015. 427(9): p. 1779-96.

158. Jankowsky, E. and M.E. Fairman, RNA helicases--one fold for many functions. Curr Opin Struct Biol, 2007. 17(3): p. 316-24.

159. Linder, P., P.F. Lasko, M. Ashburner, P. Leroy, P.J. Nielsen, K. Nishi, J. Schnier, and P.P. Slonimski, Birth of the D-E-A-D box. Nature, 1989. 337(6203): p. 121-2.

160. Schmid, S.R. and P. Linder, D-E-A-D protein family of putative RNA helicases. Mol Microbiol, 1992. 6(3): p. 283-91.

161. Linder, P. and F.V. Fuller-Pace, Looking back on the birth of DEAD-box RNA helicases. Biochim Biophys Acta, 2013. 1829(8): p. 750-5.

162. Grallert, B., S.E. Kearsey, M. Lenhard, C.R. Carlson, P. Nurse, E. Boye, and K. Labib, A fission yeast general translation factor reveals links between protein synthesis and cell cycle controls. J Cell Sci, 2000. 113(8): p. 1447-58.

163. Rocak, S. and P. Linder, DEAD-box proteins: the driving forces behind RNA metabolism. Nat Rev Mol Cell Biol, 2004. 5(3): p. 232-41.

164. Linder, P. and E. Jankowsky, From unwinding to clamping - the DEAD box RNA helicase family. Nat Rev Mol Cell Biol, 2011. 12(8): p. 505-16.

165. Jarmoskaite, I. and R. Russell, DEAD-box proteins as RNA helicases and chaperones. Wiley Interdiscip Rev RNA, 2011. 2(1): p. 135-52.

166. Pyle, A.M., Translocation and unwinding mechanisms of RNA and DNA helicases. Annu Rev Biophys, 2008. 37: p. 317-36.

167. Gustafson, E.A. and G.M. Wessel, DEAD-box helicases: posttranslational regulation and function. Biochem Biophys Res Commun, 2010. 395(1): p. 1-6.

168. Singleton, M.R., M.S. Dillingham, and D.B. Wigley, Structure and mechanism of helicases and nucleic acid translocases. Annu Rev Biochem, 2007. 76: p. 23-50.

169. Fairman-Williams, M.E., U.P. Guenther, and E. Jankowsky, SF1 and SF2 helicases: family matters. Curr Opin Struct Biol, 2010. 20(3): p. 313-24.

170. Mallam, A.L., M. Del Campo, B. Gilman, D.J. Sidote, and A.M. Lambowitz, Structural basis for RNA-duplex recognition and unwinding by the DEAD-box helicase Mss 116p. Nature, 2012. 490(7418): p. 121-5.

171. Yedavalli, V.S., C. Neuveut, Y.H. Chi, L. Kleiman, and K.T. Jeang, Requirement of DDX3 DEAD box RNA helicase for HIV-1 Rev-RRE export function. Cell, 2004. 119(3): p. 381-92.

172. Chao, C.H., C.M. Chen, P.L. Cheng, J.W. Shih, A.P. Tsou, and Y.H. Lee, DDX3, a DEAD box RNA helicase with tumor growth-suppressive property and transcriptional regulation activity of the 221 wafl/cipl promoter, is a candidate tumor suppressor. Cancer Res, 2006. 66(13): p. 6579-88.

173. Merz, C., H. Urlaub, C.L. Will, and R. Luhrmann, Protein composition of human $m R N P s$ spliced in vitro and differential requirements for $m R N P$ protein recruitment. Rna, 2007. 13(1): p. 116-28.

174. Lai, M.C., W.C. Chang, S.Y. Shieh, and W.Y. Tarn, DDX3 regulates cell growth through translational control of cyclin E1. Mol Cell Biol, 2010. 30(22): p. 544453.

175. Lai, M.C., Y.H. Lee, and W.Y. Tarn, The DEAD-box RNA helicase DDX3 associates with export messenger ribonucleoproteins as well as tip-associated 
protein and participates in translational control. Mol Biol Cell, 2008. 19(9): p. 3847-58.

176. Soto-Rifo, R., P.S. Rubilar, T. Limousin, S. de Breyne, D. Decimo, and T. Ohlmann, DEAD-box protein DDX3 associates with eIF4F to promote translation of selected mRNAs. Embo j, 2012. 31(18): p. 3745-56.

177. Soto-Rifo, R., P.S. Rubilar, and T. Ohlmann, The DEAD-box helicase DDX3 substitutes for the cap-binding protein eIF4E to promote compartmentalized translation initiation of the HIV-1 genomic RNA. Nucleic Acids Res, 2013. 41(12): p. 6286-99.

178. Robert, F. and J. Pelletier, Perturbations of RNA helicases in cancer. Wiley Interdiscip Rev RNA, 2013. 4(4): p. 333-49.

179. Martin, R., A.U. Straub, C. Doebele, and M.T. Bohnsack, DExD/H-box RNA helicases in ribosome biogenesis. RNA Biol, 2013. 10(1): p. 4-18.

180. Oh, S., R.A. Flynn, S.N. Floor, J. Purzner, L. Martin, B.T. Do, S. Schubert, D. Vaka, S. Morrissy, Y. Li, M. Kool, V. Hovestadt, D.T. Jones, P.A. Northcott, T. Risch, H.J. Warnatz, M.L. Yaspo, C.M. Adams, R.D. Leib, M. Breese, M.A. Marra, D. Malkin, P. Lichter, J.A. Doudna, S.M. Pfister, M.D. Taylor, H.Y. Chang, and Y.J. Cho, Medulloblastoma-associated DDX3 variant selectively alters the translational response to stress. Oncotarget, 2016. 7(19): p. 28169-82.

181. Valentin-Vega, Y.A., Y.D. Wang, M. Parker, D.M. Patmore, A. Kanagaraj, J. Moore, M. Rusch, D. Finkelstein, D.W. Ellison, R.J. Gilbertson, J. Zhang, H.J. Kim, and J.P. Taylor, Cancer-associated DDX3X mutations drive stress granule assembly and impair global translation. Sci Rep, 2016. 6(25996): p. 1-16.

182. Northcott, P.A., A. Korshunov, H. Witt, T. Hielscher, C.G. Eberhart, S. Mack, E. Bouffet, S.C. Clifford, C.E. Hawkins, P. French, J.T. Rutka, S. Pfister, and M.D. Taylor, Medulloblastoma comprises four distinct molecular variants. J Clin Oncol, 2011. 29(11): p. 1408-14.

183. Louis, D.N., A. Perry, G. Reifenberger, A. von Deimling, D. Figarella-Branger, W.K. Cavenee, H. Ohgaki, O.D. Wiestler, P. Kleihues, and D.W. Ellison, The 2016 World Health Organization Classification of Tumors of the Central Nervous System: a summary. Acta Neuropathol, 2016. 131(6): p. 803-20.

184. Schroeder, K. and S. Gururangan, Molecular variants and mutations in medulloblastoma. Pharmgenomics Pers Med, 2014. 2014(7): p. 43-51.

185. Chintagumpala, M. and A. Gajjar, Brain tumors. Pediatr Clin North Am, 2015. 62(1): p. 167-78.

186. Roussel, M.F. and M.E. Hatten, Cerebellum development and medulloblastoma. Curr Top Dev Biol, 2011. 94: p. 235-82.

187. Mulhern, R.K., S.L. Palmer, T.E. Merchant, D. Wallace, M. Kocak, P. Brouwers, K. Krull, M. Chintagumpala, R. Stargatt, D.M. Ashley, V.L. Tyc, L. Kun, J. Boyett, and A. Gajjar, Neurocognitive consequences of risk-adapted therapy for childhood medulloblastoma. J Clin Oncol, 2005. 23(24): p. 5511-9.

188. Massimino, M., V. Biassoni, L. Gandola, M.L. Garre, G. Gatta, F. Giangaspero, G. Poggi, and S. Rutkowski, Childhood medulloblastoma. Crit Rev Oncol Hematol, 2016. 105: p. 35-51.

189. Taylor, M.D., P.A. Northcott, A. Korshunov, M. Remke, Y.J. Cho, S.C. Clifford, C.G. Eberhart, D.W. Parsons, S. Rutkowski, A. Gajjar, D.W. Ellison, P. Lichter, 
R.J. Gilbertson, S.L. Pomeroy, M. Kool, and S.M. Pfister, Molecular subgroups of medulloblastoma: the current consensus. Acta Neuropathol, 2012. 123(4): p. 465-72.

190. Ramaswamy, V., M. Remke, E. Bouffet, S. Bailey, S.C. Clifford, F. Doz, M. Kool, C. Dufour, G. Vassal, T. Milde, O. Witt, K. von Hoff, T. Pietsch, P.A. Northcott, A. Gajjar, G.W. Robinson, L. Padovani, N. Andre, M. Massimino, B. Pizer, R. Packer, S. Rutkowski, S.M. Pfister, M.D. Taylor, and S.L. Pomeroy, Risk stratification of childhood medulloblastoma in the molecular era: the current consensus. Acta Neuropathol, 2016. 131(6): p. 821-31.

191. Northcott, P.A., D.T. Jones, M. Kool, G.W. Robinson, R.J. Gilbertson, Y.J. Cho, S.L. Pomeroy, A. Korshunov, P. Lichter, M.D. Taylor, and S.M. Pfister, Medulloblastomics: the end of the beginning. Nat Rev Cancer, 2012. 12(12): $\mathrm{p}$. 818-34.

192. Chuang, R.Y., P.L. Weaver, Z. Liu, and T.H. Chang, Requirement of the DEADBox protein dedlp for messenger RNA translation. Science, 1997. 275(5305): $\mathrm{p}$. 1468-71.

193. Lai, M.C., S.W. Wang, L. Cheng, W.Y. Tarn, S.J. Tsai, and H.S. Sun, Human DDX3 interacts with the HIV-1 Tat protein to facilitate viral mRNA translation. PLoS One, 2013. 8(7): p. e68665.

194. Craven, R.A., D.J. Griffiths, K.S. Sheldrick, R.E. Randall, I.M. Hagan, and A.M. Carr, Vectors for the expression of tagged proteins in Schizosaccharomyces pombe. Gene, 1998. 221(1): p. 59-68.

195. Sinha, I., L. Buchanan, M. Ronnerblad, C. Bonilla, M. Durand-Dubief, A. Shevchenko, M. Grunstein, A.F. Stewart, and K. Ekwall, Genome-wide mapping of histone modifications and mass spectrometry reveal $\mathrm{H} 4$ acetylation bias and H3K36 methylation at gene promoters in fission yeast. Epigenomics, 2010. 2(3): p. 377-93.

196. Kuo, Y.M., R.A. Henry, and A.J. Andrews, A quantitative multiplexed mass spectrometry assay for studying the kinetic of residue-specific histone acetylation. Methods, 2014. 70(2-3): p. 127-33.

197. Petrie, V.J., J.D. Wuitschick, C.D. Givens, A.M. Kosinski, and J.F. Partridge, RNA interference (RNAi)-dependent and RNAi-independent association of the Chpl chromodomain protein with distinct heterochromatic loci in fission yeast. Mol Cell Biol, 2005. 25(6): p. 2331-46.

198. Ishida, M., H. Shimojo, A. Hayashi, R. Kawaguchi, Y. Ohtani, K. Uegaki, Y. Nishimura, and J. Nakayama, Intrinsic nucleic acid-binding activity of Chp1 chromodomain is required for heterochromatic gene silencing. Mol Cell, 2012. 47(2): p. 228-41.

199. Leeds, P., S.W. Peltz, A. Jacobson, and M.R. Culbertson, The product of the yeast UPF1 gene is required for rapid turnover of mRNAs containing a premature translational termination codon. Genes Dev, 1991. 5(12a): p. 2303-14.

200. Plazas-Mayorca, M.D., B.M. Zee, N.L. Young, I.M. Fingerman, G. LeRoy, S.D. Briggs, and B.A. Garcia, One-pot shotgun quantitative mass spectrometry characterization of histones. J Proteome Res, 2009. 8(11): p. 5367-74.

201. Liu, H.Y., B.S. Nefsky, and N.C. Walworth, The Ded1 DEAD box helicase interacts with Chk1 and Cdc2. J Biol Chem, 2002. 277(4): p. 2637-43. 
202. Rhind, N., Z. Chen, M. Yassour, D.A. Thompson, B.J. Haas, N. Habib, I. Wapinski, S. Roy, M.F. Lin, D.I. Heiman, S.K. Young, K. Furuya, Y. Guo, A. Pidoux, H.M. Chen, B. Robbertse, J.M. Goldberg, K. Aoki, E.H. Bayne, A.M. Berlin, C.A. Desjardins, E. Dobbs, L. Dukaj, L. Fan, M.G. FitzGerald, C. French, S. Gujja, K. Hansen, D. Keifenheim, J.Z. Levin, R.A. Mosher, C.A. Muller, J. Pfiffner, M. Priest, C. Russ, A. Smialowska, P. Swoboda, S.M. Sykes, M. Vaughn, S. Vengrova, R. Yoder, Q. Zeng, R. Allshire, D. Baulcombe, B.W. Birren, W. Brown, K. Ekwall, M. Kellis, J. Leatherwood, H. Levin, H. Margalit, R. Martienssen, C.A. Nieduszynski, J.W. Spatafora, N. Friedman, J.Z. Dalgaard, P. Baumann, H. Niki, A. Regev, and C. Nusbaum, Comparative functional genomics of the fission yeasts. Science, 2011. 332(6032): p. 930-6.

203. Ford, J.C., F. al-Khodairy, E. Fotou, K.S. Sheldrick, D.J. Griffiths, and A.M. Carr, 14-3-3 protein homologs required for the DNA damage checkpoint in fission yeast. Science, 1994. 265(5171): p. 533-5.

204. Koch, A., K. Krug, S. Pengelley, B. Macek, and S. Hauf, Mitotic substrates of the kinase aurora with roles in chromatin regulation identified through quantitative phosphoproteomics of fission yeast. Sci Signal, 2011. 4(179): p. rs6.

205. Hansen, K.R., G. Burns, J. Mata, T.A. Volpe, R.A. Martienssen, J. Bahler, and G. Thon, Global effects on gene expression in fission yeast by silencing and RNA interference machineries. Mol Cell Biol, 2005. 25(2): p. 590-601.

206. Vogelauer, M., J. Wu, N. Suka, and M. Grunstein, Global histone acetylation and deacetylation in yeast. Nature, 2000. 408(6811): p. 495-8.

207. Nugent, R.L., A. Johnsson, B. Fleharty, M. Gogol, Y. Xue-Franzen, C. Seidel, A.P. Wright, and S.L. Forsburg, Expression profiling of S. pombe acetyltransferase mutants identifies redundant pathways of gene regulation. BMC Genomics, 2010. 11(59): p. 1-16.

208. Tanny, J.C., H. Erdjument-Bromage, P. Tempst, and C.D. Allis, Ubiquitylation of histone H2B controls RNA polymerase II transcription elongation independently of histone H3 methylation. Genes Dev, 2007. 21(7): p. 835-47.

209. Yang, X.J. and E. Seto, HATs and HDACs: from structure, function and regulation to novel strategies for therapy and prevention. Oncogene, 2007. 26(37): p. 5310-8.

210. Talbert, P.B. and S. Henikoff, Spreading of silent chromatin: inaction at a distance. Nat Rev Genet, 2006. 7(10): p. 793-803.

211. Bi, X., Heterochromatin structure: lessons from the budding yeast. IUBMB Life, 2014. 66(10): p. 657-66.

212. Hsu, H.C., C.L. Wang, M. Wang, N. Yang, Z. Chen, R. Sternglanz, and R.M. Xu, Structural basis for allosteric stimulation of Sir 2 activity by Sir4 binding. Genes Dev, 2013. 27(1): p. 64-73.

213. Rusche, L.N., A.L. Kirchmaier, and J. Rine, The establishment, inheritance, and function of silenced chromatin in Saccharomyces cerevisiae. Annu Rev Biochem, 2003. 72: p. 481-516.

214. Langley, E., M. Pearson, M. Faretta, U.M. Bauer, R.A. Frye, S. Minucci, P.G. Pelicci, and T. Kouzarides, Human SIR2 deacetylates p53 and antagonizes PML/p53-induced cellular senescence. Embo j, 2002. 21(10): p. 2383-96. 
215. Gomes, A.R., J.S. Yong, K.C. Kiew, E. Aydin, M. Khongkow, S.

Laohasinnarong, and E.W. Lam, Sirtuin1 (SIRT1) in the Acetylation of

Downstream Target Proteins. Methods Mol Biol, 2016. 1436: p. 169-88.

216. Vaquero, A., M. Scher, D. Lee, H. Erdjument-Bromage, P. Tempst, and D. Reinberg, Human SirT1 interacts with histone H1 and promotes formation of facultative heterochromatin. Mol Cell, 2004. 16(1): p. 93-105.

217. Lam, A.L., C.D. Boivin, C.F. Bonney, M.K. Rudd, and B.A. Sullivan, Human centromeric chromatin is a dynamic chromosomal domain that can spread over noncentromeric DNA. Proc Natl Acad Sci U S A, 2006. 103(11): p. 4186-91.

218. Guo, X., M. Kesimer, G. Tolun, X. Zheng, Q. Xu, J. Lu, J.K. Sheehan, J.D. Griffith, and $\mathrm{X}$. Li, The NAD(+)-dependent protein deacetylase activity of SIRT1 is regulated by its oligomeric status. Sci Rep, 2012. 2(640): p. 1-7.

219. Sengupta, N. and E. Seto, Regulation of histone deacetylase activities. J Cell Biochem, 2004. 93(1): p. 57-67.

220. Berdichevsky, A., M. Viswanathan, H.R. Horvitz, and L. Guarente, C. elegans SIR-2.1 interacts with 14-3-3 proteins to activate DAF-16 and extend life span. Cell, 2006. 125(6): p. 1165-77.

221. Berdichevsky, A. and L. Guarente, A stress response pathway involving sirtuins, forkheads and 14-3-3 proteins. Cell Cycle, 2006. 5(22): p. 2588-91.

222. Hong, E.J., J. Villen, E.L. Gerace, S.P. Gygi, and D. Moazed, A cullin E3 ubiquitin ligase complex associates with Rikl and the Clr4 histone H3-K9 methyltransferase and is required for RNAi-mediated heterochromatin formation. RNA Biol, 2005. 2(3): p. 106-11.

223. Stoica, C., J.B. Carmichael, H. Parker, J. Pare, and T.C. Hobman, Interactions between the RNA interference effector protein Agol and 14-3-3 proteins. consequences for cell cycle progression. J Biol Chem, 2006. 281(49): p. 3764651.

224. Macdonald, N., J.P. Welburn, M.E. Noble, A. Nguyen, M.B. Yaffe, D. Clynes, J.G. Moggs, G. Orphanides, S. Thomson, J.W. Edmunds, A.L. Clayton, J.A. Endicott, and L.C. Mahadevan, Molecular basis for the recognition of phosphorylated and phosphoacetylated histone h3 by 14-3-3. Mol Cell, 2005. 20(2): p. 199-211.

225. Winter, S., E. Simboeck, W. Fischle, G. Zupkovitz, I. Dohnal, K. Mechtler, G. Ammerer, and C. Seiser, 14-3-3 proteins recognize a histone code at histone H3 and are required for transcriptional activation. Embo j, 2008. 27(1): p. 88-99.

226. Dobbin, M.M., R. Madabhushi, L. Pan, Y. Chen, D. Kim, J. Gao, B. Ahanonu, P.C. Pao, Y. Qiu, Y. Zhao, and L.H. Tsai, SIRT1 collaborates with ATM and HDAC1 to maintain genomic stability in neurons. Nat Neurosci, 2013. 16(8): p. 1008-15.

227. Qiu, Y., Y. Zhao, M. Becker, S. John, B.S. Parekh, S. Huang, A. Hendarwanto, E.D. Martinez, Y. Chen, H. Lu, N.L. Adkins, D.A. Stavreva, M. Wiench, P.T. Georgel, R.L. Schiltz, and G.L. Hager, HDAC1 acetylation is linked to progressive modulation of steroid receptor-induced gene transcription. Mol Cell, 2006. 22(5): p. 669-79. 
228. Binda, O., C. Nassif, and P.E. Branton, SIRT1 negatively regulates HDAC1dependent transcriptional repression by the RBP1 family of proteins. Oncogene, 2008. 27(24): p. 3384-92.

229. Ahringer, J., NuRD and SIN3 histone deacetylase complexes in development. Trends Genet, 2000. 16(8): p. 351-6.

230. Iwahara, T., R. Bonasio, V. Narendra, and D. Reinberg, SIRT3 functions in the nucleus in the control of stress-related gene expression. Mol Cell Biol, 2012. 32(24): p. 5022-34.

231. Etchegaray, J.P., L. Zhong, and R. Mostoslavsky, The histone deacetylase SIRT6: at the crossroads between epigenetics, metabolism and disease. Curr Top Med Chem, 2013. 13(23): p. 2991-3000.

232. Kang, W.K., Y.H. Kim, B.S. Kim, and J.Y. Kim, Growth phase-dependent roles of Sir2 in oxidative stress resistance and chronological lifespan in yeast. $\mathrm{J}$ Microbiol, 2014. 52(8): p. 652-8.

233. Bosch-Presegue, L. and A. Vaquero, The dual role of sirtuins in cancer. Genes Cancer, 2011. 2(6): p. 648-62.

234. Suka, N., Y. Suka, A.A. Carmen, J. Wu, and M. Grunstein, Highly specific antibodies determine histone acetylation site usage in yeast heterochromatin and euchromatin. Mol Cell, 2001. 8(2): p. 473-9.

235. Thorne, A.W., D. Kmiciek, K. Mitchelson, P. Sautiere, and C. Crane-Robinson, Patterns of histone acetylation. Eur J Biochem, 1990. 193(3): p. 701-13.

236. Morales, V. and H. Richard-Foy, Role of histone N-terminal tails and their acetylation in nucleosome dynamics. Mol Cell Biol, 2000. 20(19): p. 7230-7.

237. Wyrick, J.J. and M.A. Parra, The role of histone H2A and H2B post-translational modifications in transcription: a genomic perspective. Biochim Biophys Acta, 2009. 1789(1): p. 37-44.

238. Fierz, B., C. Chatterjee, R.K. McGinty, M. Bar-Dagan, D.P. Raleigh, and T.W. Muir, Histone H2B ubiquitylation disrupts local and higher-order chromatin compaction. Nat Chem Biol, 2011. 7(2): p. 113-9.

239. Mao, P., M.N. Kyriss, A.J. Hodges, M. Duan, R.T. Morris, M.D. Lavine, T.B. Topping, L.M. Gloss, and J.J. Wyrick, A basic domain in the histone $\mathrm{H} 2 \mathrm{~B} \mathrm{~N}$ terminal tail is important for nucleosome assembly by FACT. Nucleic Acids Res, 2016. 44(19): p. 9142-9152.

240. Ahn, S.H., W.L. Cheung, J.Y. Hsu, R.L. Diaz, M.M. Smith, and C.D. Allis, Sterile 20 kinase phosphorylates histone H2B at serine 10 during hydrogen peroxide-induced apoptosis in S. cerevisiae. Cell, 2005. 120(1): p. 25-36.

241. Ahn, S.H., R.L. Diaz, M. Grunstein, and C.D. Allis, Histone H2B deacetylation at lysine 11 is required for yeast apoptosis induced by phosphorylation of $\mathrm{H} 2 \mathrm{~B}$ at serine 10. Mol Cell, 2006. 24(2): p. 211-20.

242. Cheung, W.L., K. Ajiro, K. Samejima, M. Kloc, P. Cheung, C.A. Mizzen, A. Beeser, L.D. Etkin, J. Chernoff, W.C. Earnshaw, and C.D. Allis, Apoptotic phosphorylation of histone $H 2 B$ is mediated by mammalian sterile twenty kinase. Cell, 2003. 113(4): p. 507-17.

243. Ajiro, K., A.B. Scoltock, L.K. Smith, M. Ashasima, and J.A. Cidlowski, Reciprocal epigenetic modification of histone H2B occurs in chromatin during apoptosis in vitro and in vivo. Cell Death Differ, 2010. 17(6): p. 984-93. 
244. Fernandez-Vazquez, J., I. Vargas-Perez, M. Sanso, K. Buhne, M. Carmona, E. Paulo, D. Hermand, M. Rodriguez-Gabriel, J. Ayte, S. Leidel, and E. Hidalgo, Modification of tRNA(Lys) UUU by elongator is essential for efficient translation of stress mRNAs. PLoS Genet, 2013. 9(7): p. e1003647.

245. Hamiche, A., J.G. Kang, C. Dennis, H. Xiao, and C. Wu, Histone tails modulate nucleosome mobility and regulate ATP-dependent nucleosome sliding by NURF. Proc Natl Acad Sci U S A, 2001. 98(25): p. 14316-21.

246. Fernandez-Capetillo, O., C.D. Allis, and A. Nussenzweig, Phosphorylation of histone H2B at DNA double-strand breaks. J Exp Med, 2004. 199(12): p. 1671-7.

247. Hogbom, M., R. Collins, S. van den Berg, R.M. Jenvert, T. Karlberg, T. Kotenyova, A. Flores, G.B. Karlsson Hedestam, and L.H. Schiavone, Crystal structure of conserved domains 1 and 2 of the human DEAD-box helicase DDX3X in complex with the mononucleotide AMP. J Mol Biol, 2007. 372(1): p. 150-9.

248. McDowall, M.D., M.A. Harris, A. Lock, K. Rutherford, D.M. Staines, J. Bahler, P.J. Kersey, S.G. Oliver, and V. Wood, PomBase 2015: updates to the fission yeast database. Nucleic Acids Res, 2015. 43(D1): p. D656-61.

249. Wood, V., M.A. Harris, M.D. McDowall, K. Rutherford, B.W. Vaughan, D.M. Staines, M. Aslett, A. Lock, J. Bahler, P.J. Kersey, and S.G. Oliver, PomBase: $a$ comprehensive online resource for fission yeast. Nucleic Acids Res, 2012. 40(D1): p. D695-9.

250. Kersey, P.J., D.M. Staines, D. Lawson, E. Kulesha, P. Derwent, J.C. Humphrey, D.S. Hughes, S. Keenan, A. Kerhornou, G. Koscielny, N. Langridge, M.D. McDowall, K. Megy, U. Maheswari, M. Nuhn, M. Paulini, H. Pedro, I. Toneva, D. Wilson, A. Yates, and E. Birney, Ensembl Genomes: an integrative resource for genome-scale data from non-vertebrate species. Nucleic Acids Res, 2012. 40(D1): p. D91-7.

251. Buchan, J.R. and R. Parker, Eukaryotic stress granules: the ins and outs of translation. Mol Cell, 2009. 36(6): p. 932-41.

252. Mamane, Y., E. Petroulakis, L. Rong, K. Yoshida, L.W. Ler, and N. Sonenberg, eIF4E--from translation to transformation. Oncogene, 2004. 23(18): p. 3172-9.

253. Silvera, D., S.C. Formenti, and R.J. Schneider, Translational control in cancer. Nat Rev Cancer, 2010. 10(4): p. 254-66.

254. Gingras, A.C., B. Raught, and N. Sonenberg, eIF4 initiation factors: effectors of $m R N A$ recruitment to ribosomes and regulators of translation. Annu Rev Biochem, 1999. 68: p. 913-63.

255. Wendel, H.G., R.L. Silva, A. Malina, J.R. Mills, H. Zhu, T. Ueda, R. WatanabeFukunaga, R. Fukunaga, J. Teruya-Feldstein, J. Pelletier, and S.W. Lowe, Dissecting eIF4E action in tumorigenesis. Genes Dev, 2007. 21(24): p. 3232-7.

256. Koromilas, A.E., A. Lazaris-Karatzas, and N. Sonenberg, mRNAs containing extensive secondary structure in their 5' non-coding region translate efficiently in cells overexpressing initiation factor eIF-4E. Embo j, 1992. 11(11): p. 4153-8.

257. Shih, J.W., T.Y. Tsai, C.H. Chao, and Y.H. Wu Lee, Candidate tumor suppressor DDX3 RNA helicase specifically represses cap-dependent translation by acting as an eIF4E inhibitory protein. Oncogene, 2008. 27(5): p. 700-14.

258. Botlagunta, M., F. Vesuna, Y. Mironchik, A. Raman, A. Lisok, P. Winnard, Jr., S. Mukadam, P. Van Diest, J.H. Chen, P. Farabaugh, A.H. Patel, and V. Raman, 
Oncogenic role of DDX3 in breast cancer biogenesis. Oncogene, 2008. 27(28): $\mathrm{p}$. 3912-22.

259. Wu, D.W., W.S. Liu, J. Wang, C.Y. Chen, Y.W. Cheng, and H. Lee, Reduced p21(WAF1/CIP1) via alteration of p53-DDX3 pathway is associated with poor relapse-free survival in early-stage human papillomavirus-associated lung cancer. Clin Cancer Res, 2011. 17(7): p. 1895-905.

260. Bol, G.M., M. Xie, and V. Raman, DDX3, a potential target for cancer treatment. Mol Cancer, 2015. 14(188): p. 1-16.

261. Bol, G.M., F. Vesuna, M. Xie, J. Zeng, K. Aziz, N. Gandhi, A. Levine, A. Irving, D. Korz, S. Tantravedi, M.R. Heerma van Voss, K. Gabrielson, E.A. Bordt, B.M. Polster, L. Cope, P. van der Groep, A. Kondaskar, M.A. Rudek, R.S. Hosmane, E. van der Wall, P.J. van Diest, P.T. Tran, and V. Raman, Targeting DDX3 with a small molecule inhibitor for lung cancer therapy. EMBO Mol Med, 2015. 7(5): p. 648-69.

262. Floor, S.N., K.J. Barkovich, K.J. Condon, K.M. Shokat, and J.A. Doudna, Analog sensitive chemical inhibition of the DEAD-box protein DDX3. Protein Sci, 2016. 25(3): p. 638-49.

263. Jiang, L., Z.H. Gu, Z.X. Yan, X. Zhao, Y.Y. Xie, Z.G. Zhang, C.M. Pan, Y. Hu, C.P. Cai, Y. Dong, J.Y. Huang, L. Wang, Y. Shen, G. Meng, J.F. Zhou, J.D. Hu, J.F. Wang, Y.H. Liu, L.H. Yang, F. Zhang, J.M. Wang, Z. Wang, Z.G. Peng, F.Y. Chen, Z.M. Sun, H. Ding, J.M. Shi, J. Hou, J.S. Yan, J.Y. Shi, L. Xu, Y. Li, J. Lu, Z. Zheng, W. Xue, W.L. Zhao, Z. Chen, and S.J. Chen, Exome sequencing identifies somatic mutations of DDX3X in natural killer/T-cell lymphoma. Nat Genet, 2015. 47(9): p. 1061-6.

264. Ojha, J., C.R. Secreto, K.G. Rabe, D.L. Van Dyke, K.M. Kortum, S.L. Slager, T.D. Shanafelt, R. Fonseca, N.E. Kay, and E. Braggio, Identification of recurrent truncated DDX 3 X mutations in chronic lymphocytic leukaemia. Br J Haematol, 2015. 169(3): p. 445-8.

265. He, T.Y., D.W. Wu, P.L. Lin, L. Wang, C.C. Huang, M.C. Chou, and H. Lee, DDX3 promotes tumor invasion in colorectal cancer via the CKlepsilon/Dvl2 axis. Sci Rep, 2016. 6(21483): p. 1-10.

266. Majewska, H., A. Skalova, I. Weinreb, D. Stodulski, M. Hyrcza, C. Stankiewicz, and W. Biernat, Giant cribriform adenocarcinoma of the tongue showing PRKD3 rearrangement. Pol J Pathol, 2016. 67(1): p. 84-90.

267. Joseph, N.M., Y.Y. Chen, A. Nasr, I. Yeh, E. Talevich, C. Onodera, B.C. Bastian, J.T. Rabban, K. Garg, C. Zaloudek, and D.A. Solomon, Genomic profiling of malignant peritoneal mesothelioma reveals recurrent alterations in epigenetic regulatory genes BAP1, SETD2, and DDX3X. Mod Pathol, 2017. 30(2): p. 246254. 
APPENDIX. CHAPTER 2 SUPPLEMENTAL TABLES 
Table A-1. List of strains used in this study.

\begin{tabular}{|c|c|c|}
\hline Strain & Genotype & Source \\
\hline PY41 & $\mathrm{h}+$ ade6-210 arg3-D4 his3-D1 leu1-32 ura4-D18 & Lab Stock \\
\hline PY42 & h- ade6-210 arg3-D4 his3-D1 leu1-32 ura4-D18 & Lab Stock \\
\hline PY1550 & h- dcr $1 \Delta::$ KanR ade6-210 leul-32 ade6-210 ura4DS/E & Lab Stock \\
\hline PY1798 & $\begin{array}{l}\text { h- clr4 } 4:: \text { KanR ade6-210 leu1-32 arg3-D his3-D1 } \\
\text { ura4DS/E }\end{array}$ & Lab Stock \\
\hline PY1836 & $\begin{array}{l}\text { h- } \operatorname{clr} 44:: \operatorname{KanR} \text { otr } 1 R(\operatorname{Sph} 1):: \text { ura }^{+} \text {ade6-210 his3-D1 } \\
\text { leu1-32 ura4-DS/E}\end{array}$ & Lab Stock \\
\hline PY2036 & h- otr1R (Sph1)::ura4 $4^{+}$ade6-210 leu1-32 ura4-DS/E & Lab Stock \\
\hline PY2941 & $\begin{array}{l}\text { h- } \operatorname{clr} 34:: \text { kanMX6 otr } 1 R \text { (Sph1):: }{\text { ura } 4^{+}} \text {ade6-210 his } 3^{+} \\
\text {leu1-32 ura4-DS/E }\end{array}$ & Lab Stock \\
\hline PY2942 & h90 clr3A::kanMX6 ade6-210 his $3 D$ leu1-32 ura4 DS/E & Lab Stock \\
\hline PY4765 & $\begin{array}{l}\text { h- } \operatorname{sir} 24:: \text { kanMX6 otr } 1 R \text { (Sph1)::ura } 4^{+} \text {ura } 4-D S / E \text { ade6- } \\
210 \text { leu1-32 his3-D }\end{array}$ & 83 \\
\hline PY4783 & $\begin{array}{l}\text { h- clr } 4^{+}-\text {his } 3^{+}[\mathrm{JP} 1084(\mathrm{HpaI})]:: \text { clr } 4 \Delta:: \text { KanR otr } 1 R \\
\left(\text { Sph1)::ura } 4^{+} \text {ade6-210 his } 3-D \text { leu1-32 ura4-DS/E }\right.\end{array}$ & 83 \\
\hline PY5088 & $\begin{array}{l}\text { h- } \operatorname{sir} 24:: \operatorname{kanMX6} \operatorname{clr} 34:: \operatorname{kanMX6} \text { otr } 1 R(\operatorname{Sph} 1): \because \mathrm{ura}^{+}{ }^{+} \\
\text {his } 3-D \text { leu } 1-32 \text { ade } 6-210^{+} \text {ura } 4 \mathrm{DS} / \mathrm{E}\end{array}$ & 83 \\
\hline PY5090 & $\begin{array}{l}\text { h- } \operatorname{sir} 2 \Delta:: \text { kanMX6 clr } 4 \Delta:: \text { KanR }:: \text { clr } 4+\text {-ade } 6+ \\
\text { otr1R(SphI) }: \because \text { ura }^{+} \text {ade6-210 his3-D leu1-32 ura4-DS/E }\end{array}$ & 83 \\
\hline PY6120 & $\begin{array}{l}\text { h- otr1R (Sph1)::ura4 }{ }^{+} \text {ade6-210 leul-32 ura4-DS/E + } \\
\text { [JP1611 pREP41-3x V5] }\end{array}$ & 83 \\
\hline PY6158 & $\begin{array}{l}\text { h- clr44::KanR otr1R (Sph1):: } \text { ura }^{+} \text {ade6-210 his3-D1 } \\
\text { leu1-32 ura4-DS/E + [JP1611 pREP41-3x V5] }\end{array}$ & 83 \\
\hline PY7803 & h- ded1-61 cig2-3x HA leu1-32 & 162 \\
\hline PY7805 & h- ded1-1D5 cig2-3x HA & 162 \\
\hline PY7885 & h- ded1-1D5 cig2-3x HA leu1-32 & 157 \\
\hline PY8049 & $\begin{array}{l}\text { h- ded1-1D5 cig2-3x HA leu1-32 + [JP2102 pREP41- } \\
\text { Ded1-3x V5] }\end{array}$ & 157 \\
\hline PY8050 & $\begin{array}{l}\text { h- ded1-1D5 cig2-3x HA leu1-32 + [JP2102 pREP41- } \\
\text { Ded1-3x V5] }\end{array}$ & 157 \\
\hline PY8053 & $\begin{array}{l}\text { h- ded1-1D5 cig2-3x HA leu1-32 + [JP2104 pREP41- } \\
\text { DDX3X-3x V5] }\end{array}$ & 157 \\
\hline PY8054 & $\begin{array}{l}\text { h- ded1-1D5 cig2-3x HA leu1-32 + [JP2104 pREP41- } \\
\text { DDX3X-3x V5] }\end{array}$ & 157 \\
\hline PY8108 & $\begin{array}{l}\text { h- ded1-1D5 cig2-3x HA leu1-32 + [JP2116 pREP41- } \\
\text { DDX3X G302V-3x V5] }\end{array}$ & 157 \\
\hline PY8111 & $\begin{array}{l}\text { h- ded1-1D5 cig2-3x HA leu1-32 + [JP2132 pREP41- } \\
\text { DDX3X A222P-3x V5] }\end{array}$ & 157 \\
\hline PY8115 & $\begin{array}{l}\text { h- ded1-1D5 cig2-3x HA leu1-32 + [JP2133 pREP41- } \\
\text { DDX3X G325E-3x V5] }\end{array}$ & 157 \\
\hline PY8119 & $\begin{array}{l}\text { h- ded1-1D5 cig2-3x HA leu1-32 + [JP2134 pREP41- } \\
\text { DDX3X T275M-3x V5] }\end{array}$ & 157 \\
\hline
\end{tabular}


Table A-1. (Continued).

\begin{tabular}{|c|c|c|}
\hline Strain & Genotype & Source \\
\hline PY8123 & $\begin{array}{l}\text { h- ded1-1D5 cig2-3x HA leu1-32 + [JP2135 pREP41- } \\
\text { DDX3X R351W-3x V5] }\end{array}$ & 157 \\
\hline PY8127 & $\begin{array}{l}\text { h- ded1-1D5 cig2-3x HA leu1-32 + [JP2136 pREP41 } \\
\text { P568L-DDX3X-3x V5] }\end{array}$ & 157 \\
\hline PY8131 & $\begin{array}{l}\text { h- ded1-1D5 cig2-3x HA leu1-32 + [JP2137 pREP41 } \\
\text { L353F-DDX3X-3x V5] }\end{array}$ & 157 \\
\hline PY8135 & $\begin{array}{l}\mathrm{h}-\text { ded1-1D5 cig2-3x HA leu1-32 + [JP2138 pREP41- } \\
\text { DDX3X K230A-3x V5] }\end{array}$ & 157 \\
\hline PY8139 & $\begin{array}{l}\mathrm{h}-\text { ded1-1D5 cig2-3x HA leu1-32 + [JP2140 pREP41- } \\
\text { DDX3X D354V-3x V5] }\end{array}$ & 157 \\
\hline PY8183 & $\begin{array}{l}\text { h- ded1-1D5 cig2-3x HA leul-32 + [JP2139 pREP41- } \\
\text { DDX3X M370R-3x V5] }\end{array}$ & 157 \\
\hline PY8300 & h- ded1-61 cig2-3x HA leu1-32 + [JP918 pREP81-3x HA] & 157 \\
\hline PY8303 & $\begin{array}{l}\text { h- ded1-61 cig2-3x HA leu1-32 + [JP2102 pREP41-Ded1- } \\
3 \mathrm{x} \text { V5] }\end{array}$ & 157 \\
\hline PY8306 & $\begin{array}{l}\text { h- ded1-61 cig2-3x HA leu1-32 + [JP2104 pREP41- } \\
\text { DDX3X-3x V5] }\end{array}$ & 157 \\
\hline PY8309 & $\begin{array}{l}\text { h- ded1-61 cig2-3x HA leu1-32 + [JP2116 pREP41- } \\
\text { DDX3X G302V-3x V5] }\end{array}$ & 157 \\
\hline PY8312 & $\begin{array}{l}\text { h- ded1-61 cig2-3x HA leu1-32 + [JP2132 pREP41- } \\
\text { DDX3X A222P-3x V5] }\end{array}$ & 157 \\
\hline PY8315 & $\begin{array}{l}\text { h- ded1-61 cig2-3x HA leu1-32 + [JP2134 pREP41- } \\
\text { DDX3X G325E-3x V5] }\end{array}$ & 157 \\
\hline PY8318 & $\begin{array}{l}\text { h- ded1-61 cig2-3x HA leu1-32 + [JP2134 pREP41- } \\
\text { DDX3X T275M-3x V5] }\end{array}$ & 157 \\
\hline PY8321 & $\begin{array}{l}\text { h- ded1-61 cig2-3x HA leu1-32 + [JP2135 pREP41- } \\
\text { DDX3X R351W-3x V5] }\end{array}$ & 157 \\
\hline PY 8324 & $\begin{array}{l}\text { h- ded1-61 cig2-3x HA leul-32 + [JP2136 pREP41 P568L- } \\
\text { DDX3X-3x V5] }\end{array}$ & 157 \\
\hline PY8327 & $\begin{array}{l}\text { h- ded1-61 cig2-3x HA leu1-32 + [JP2137 pREP41 L353F- } \\
\text { DDX3X-3x V5] }\end{array}$ & 157 \\
\hline PY8330 & $\begin{array}{l}\text { h- ded1-61 cig2-3x HA leu1-32 + [JP2138 pREP41- } \\
\text { DDX3X K230A-3x V5] }\end{array}$ & 157 \\
\hline PY8333 & $\begin{array}{l}\text { h- ded1-61 cig2-3x HA leu1-32 + [JP2139 pREP41- } \\
\text { DDX3X M370R-3x V5] }\end{array}$ & 157 \\
\hline PY8336 & $\begin{array}{l}\text { h- ded1-61 cig2-3x HA leu1-32 + [JP2140 pREP41- } \\
\text { DDX3X D354V-3x V5] }\end{array}$ & 157 \\
\hline PY9047 & $\begin{array}{l}\text { h- } \operatorname{sir} 24:: k a n M X 6 \text { clr4D }:: K a n R:: c l r 4^{+}-\text {ade }^{+} \\
\text {otr1R(SphI)::ura4 }{ }^{+} \text {ade6-210 his3-D leul-32 ura4-DS/E+ } \\
{[\text { JP1611 pREP81-3x V5] }}\end{array}$ & This Study \\
\hline PY9202 & $\begin{array}{l}\text { h- } \operatorname{sir} 2 \Delta:: \text { kanMX6 clr4D }: \text { KanR }:: \operatorname{clr} 4^{+}-\text {ade }^{+} \\
\text {otr1R(SphI)::ura4 }{ }^{+} \text {ade6-210 his3-D leul-32 ura4-DS/E+ } \\
{[\text { JP2302 pREP81-Sir2-3x V5] }}\end{array}$ & This Study \\
\hline
\end{tabular}


Table A-1. (Continued).

\begin{tabular}{|c|c|c|}
\hline Strain & Genotype & Source \\
\hline PY9207 & $\begin{array}{l}\text { h- } \operatorname{sir} 2 \Delta:: k a n M X 6 \text { clr4D }:: K a n R:: c l r 4^{+}-\text {ade }^{+} \\
\text {otr1R(SphI)::ura4 }{ }^{+} \text {ade6-210 his3-D leul-32 ura4-DS/E+ } \\
{[\text { JP2304 pREP81-Sir2 N247A-3x V5] }}\end{array}$ & This Study \\
\hline PY9212 & 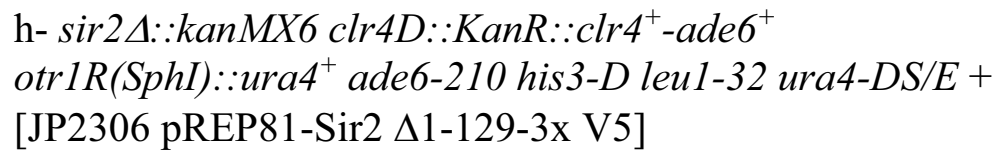 & This Study \\
\hline PY9216 & $\begin{array}{l}\text { h- } \operatorname{sir} 2 \Delta:: k a n M X 6 \text { clr4D }:: K a n R:: c l r 4^{+}-\text {ade }^{+} \\
\text {otr1R(SphI)::ura4 }{ }^{+} \text {ade6-210 his 3-D leul-32 ura4-DS/E+ } \\
{[\text { JP2306 pREP81-Sir2 } \Delta 1-129 \text { N247A-3x V5] }}\end{array}$ & This Study \\
\hline PY9220 & $\begin{array}{l}\text { h- sir2 } \Delta:: \text { kanMX6 otr1R (Sph1)::ura4 }{ }^{+} \text {ura4-DS/E ade6- } \\
210 \text { leu1-32 his3-D + [JP1611 pREP81-3x V5] }\end{array}$ & This Study \\
\hline PY9222 & $\begin{array}{l}\text { h- sir24::kanMX6 otr 1R (Sph1)::ura4 }{ }^{+} \text {ura4-DS/E ade6- } \\
210 \text { leu1-32 his3-D + [JP2302 pREP81-Sir2-3x V5] }\end{array}$ & This Study \\
\hline PY9224 & $\begin{array}{l}\text { h- sir24::kanMX6 otr 1R (Sph1)::ura4 } 4^{+} \text {ura } 4-D S / E \text { ade6- } \\
210 \text { leu1-32 his3-D + [JP2304 pREP81-Sir2 N247A-3x } \\
\text { V5] }\end{array}$ & This Study \\
\hline PY9226 & $\begin{array}{l}\text { h- sir2 } 4:: k a n M X 6 \text { otr } 1 R \text { (Sph1)::ura4 } 4^{+} \text {ura } 4-D S / E \text { ade6- } \\
210 \text { leu1-32 his3-D + [JP2306 pREP81-Sir2 } \Delta 1-129-3 \mathrm{x} \\
\text { V5] }\end{array}$ & This Study \\
\hline PY9228 & $\begin{array}{l}\text { h- sir2 } 4: \text { kanMX6 otr } 1 R \text { (Sph1)::ura4 } 4^{+} \text {ura } 4-D S / E \text { ade6- } \\
210 \text { leu1-32 his3-D + [JP2308 pREP81-Sir2 } \Delta 1-129 \\
\text { N247A-3x V5] }\end{array}$ & This Study \\
\hline PY9395 & h- htb1 K5,6,10,15Q-Flag-Kan arg3D his3-D leu1-32 & This Study \\
\hline PY9396 & h- htb1 K5,6,10,15Q-Flag-Kan arg3D his3-D leu1-32 & This Study \\
\hline PY9412 & $\begin{array}{l}\text { h- htbl K5,6,10,15Q-Flag-Kan clr4 } 4:: \text { KanR otr1R } \\
\left(\text { Sph1)::ura } 4^{+} \text {his3-D leul-32 }\right.\end{array}$ & This Study \\
\hline PY9505 & $\begin{array}{l}\mathrm{h}+/ \mathrm{h} 90 \mathrm{htb} 1 \mathrm{~K} 5,6,10,15 Q-F l a g-K a n \text { mat3-M::ura4 }{ }^{+} \text {leu1- } \\
32 \text { ura4-DS/E }\end{array}$ & This Study \\
\hline PY9506 & $\begin{array}{l}\mathrm{h}+/ \mathrm{h} 90 \text { htb1 K5,6,10,15Q-Flag-Kan mat3-M::ura4 }{ }^{+} \text {leu1- } \\
32 \text { ura4-DS/E }\end{array}$ & This Study \\
\hline PY9509 & $\begin{array}{l}\text { h- htb1 K5,6,10,15Q-Flag-Kan clr4 } 4^{+} \text {his } 3^{+}[\mathrm{JP} 1084 \\
\text { (HpaI)]::clr4 }:: \text { KanR otr1R Sph1::ura4 his3-D leu1-32 }\end{array}$ & This Study \\
\hline PY9510 & $\begin{array}{l}\text { h- htb1 K5,6,10,15Q-Flag-Kan clr }{ }^{+}-\text {his } 3^{+}[\mathrm{JP} 1084 \\
\text { (HpaI)]::clr4 }:: \text { KanR otr1R Sph1::ura4 his 3-D leul-32 }\end{array}$ & This Study \\
\hline PY9519 & h- htb1 K5,6,10,15R-Flag-Kan arg-D his3-D leu1-32 & This Study \\
\hline PY9520 & h- htb1 K5,6,10,15R-Flag-Kan arg-D his3-D leu1-32 & This Study \\
\hline PY9586 & 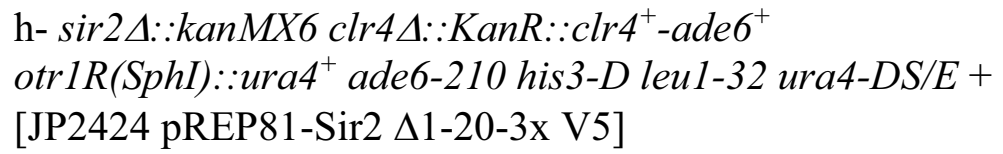 & This Study \\
\hline PY9598 & $\begin{array}{l}\text { h- } \operatorname{sir} 2 \Delta:: \text { kanMX6 clr4 } \Delta:: \text { KanR: }: \text { clr }^{+}{ }^{+} \text {-ade } 6^{+} \\
\text {otr1R(SphI)::ura4 }{ }^{+} \text {ade6-210 his3-D leu1-32 ura4-DS/E+ } \\
{[\text { JP2426 pREP81-Sir2 } \Delta 1-20 \text { N247A-3x V5] }}\end{array}$ & This Study \\
\hline
\end{tabular}


Table A-1. (Continued).

\begin{tabular}{|c|c|c|}
\hline Strain & Genotype & Source \\
\hline PY9600 & 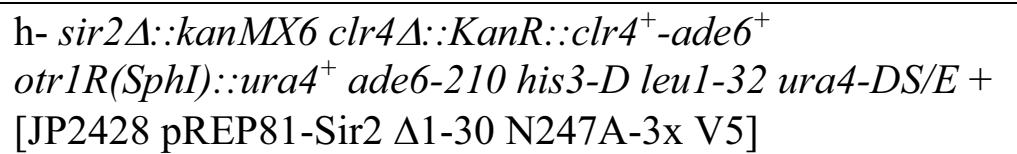 & This Study \\
\hline PY9602 & 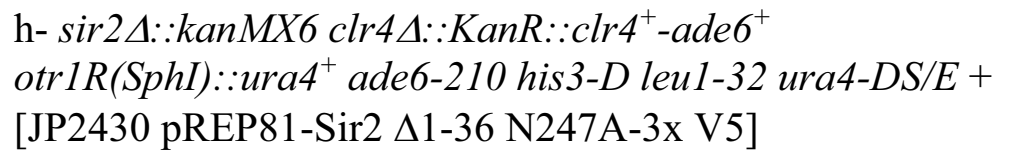 & This Study \\
\hline PY9604 & $\begin{array}{l}\text { h- } \operatorname{sir} 2 \Delta:: \text { kanMX6 clr4 } \Delta:: \text { KanR }:: \text { clr }^{+}-\text {ade }^{+} \\
\text {otr1R(SphI) }: \because \text { ura }^{+} \text {ade6-210 his } 3-D \text { leu1-32 ura4-DS/E+ } \\
{[\text { JP2434 pREP81-Sir2 } \Delta 1-60 \text { N247A-3x V5] }}\end{array}$ & This Study \\
\hline PY9606 & $\begin{array}{l}\text { h- } \operatorname{sir} 2 \Delta:: \text { kanMX6 clr4 } \Delta:: \text { KanR }:: \text { clr }^{+}-\text {ade }^{+} \\
\text {otr1R(SphI) }:: \text { ura }^{+} \text {ade6-210 his3-D leu1-32 ura4-DS/E+ } \\
{[\text { JP2438 pREP81-Sir2 } \Delta 1-95 \text { N247A-3x V5] }}\end{array}$ & This Study \\
\hline PY9608 & 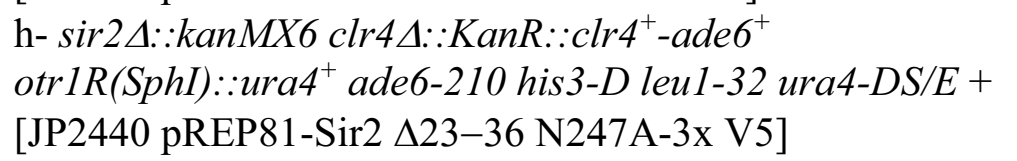 & This Study \\
\hline PY9657 & h- htb1 K5,6,10,15Q arg3D his3-D leu1-32 ura4-D18 & This Study \\
\hline PY9658 & h- htb1 K5,6,10,15Q arg3D his3-D leu1-32 ura4-D18 & This Study \\
\hline PY9659 & h- htb1 K5,6,10,15Q arg3D his3-D leu1-32 ura4-D18 & This Study \\
\hline PY9662 & $\begin{array}{l}\text { h- clr34::kanMX6 ade6-210 arg3-D his3-D leul-32 ura4- } \\
\text { D18 }\end{array}$ & 149 \\
\hline PY9663 & $\begin{array}{l}\text { h- clr34::kanMX6 ade6-210 arg3-D his3-D leu1-32 ura4- } \\
\text { D18 }\end{array}$ & 149 \\
\hline PY9666 & h- clr1 $1:: K a n M X 4$ arg3-D his3-D leu1-32 ura4-D18 & 149 \\
\hline PY9667 & h- $\operatorname{clr} 1 \Delta:: K a n M X 4$ arg3-D his3-D leu1-32 ura4-D18 & 149 \\
\hline PY9671 & h- $\operatorname{clr} 2 \Delta::$ his $7^{+}$ade6-216 arg3-D his3-D leu1-32 ura4-D18 & 149 \\
\hline PY9672 & h- $\operatorname{clr} 2 \Delta::$ his $7^{+}$ade6-216 arg3-D his3-D leu1-32 ura4-D18 & 149 \\
\hline PY9720 & 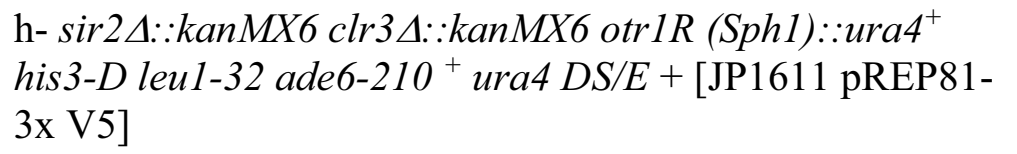 & This Study \\
\hline PY9723 & 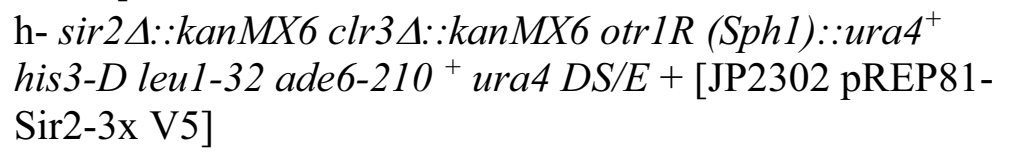 & This Study \\
\hline PY9726 & 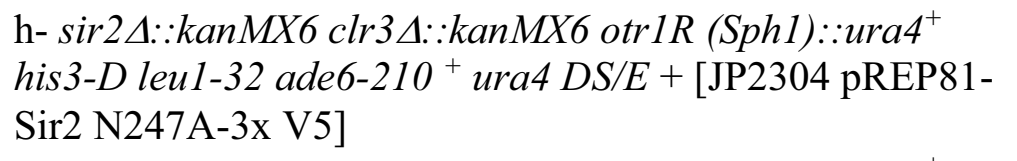 & This Study \\
\hline PY9729 & $\begin{array}{l}\text { h- sir2 } \Delta:: \text { kanMX6 } \operatorname{clr} 3 \Delta:: k a n M X 6 \text { otr } 1 R(\text { Sph1 }):: \text { ura }^{+} \\
\text {his3-D leu1-32 ade6-210 } \\
\text { Sir2 } \Delta 1-129-3 \mathrm{x} \text { V5] }\end{array}$ & This Study \\
\hline PY9732 & 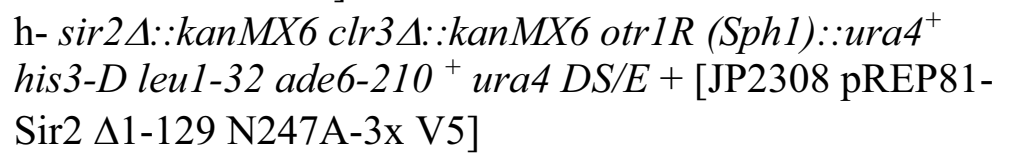 & This Study \\
\hline
\end{tabular}


Table A-1. (Continued).

\begin{tabular}{|c|c|c|}
\hline Strains & Genotype & Source \\
\hline PY9841 & $\begin{array}{l}\text { h- mit1 } 1:: N a t M X 6 \text { ade6-210 arg3-D4 his3-D1 leu1-32 } \\
\text { ura4-D18 }\end{array}$ & 149 \\
\hline PY9842 & $\begin{array}{l}\text { h- mit1 } \Delta:: N a t M X 6 \text { ade6-210 arg3-D4 his3-D1 leu1-32 } \\
\text { ura4-D18 }\end{array}$ & 149 \\
\hline PY9844 & $\begin{array}{l}\text { h- chp24::KanR ade6-210 arg3-D4 his3-D1 leu1-32 ura4- } \\
\text { D18 }\end{array}$ & 149 \\
\hline PY9845 & $\begin{array}{l}\text { h- chp24::KanR ade6-210 arg3-D4 his3-D1 leu1-32 ura4- } \\
\text { D18 }\end{array}$ & 149 \\
\hline PY9860 & $\begin{array}{l}\text { h- sir2 } 4:: k a n M X 6 \text { ade6-210 arg3-D his3-D leu1-32 ura4- } \\
\text { D18 }\end{array}$ & This Study \\
\hline PY9861 & $\begin{array}{l}\text { h- sir24::kanMX6 ade6-210 arg3-D his3-D leu1-32 ura4- } \\
\text { D18 }\end{array}$ & This Study \\
\hline PY9918 & $\begin{array}{l}\text { h- sir2 } \Delta:: k a n M X 6 \text { otr } 1 R(S p h 1):: \text { ura }^{+} \text {ura } 4-D S / E \text { ade6- } \\
210 \text { leul-32 his } 3-D+[\mathrm{JP} 2424 \text { Prep81-Sir2 } \Delta 1-20-3 \mathrm{x} \text { V5] }\end{array}$ & This Study \\
\hline PY9964 & $\begin{array}{l}\text { h- } \operatorname{sir} 2 \Delta:: \text { kanMX6 clr4 } 4:: \text { KanR }:: c l r 4^{+} \text {-ade } 6^{+} \\
\text {otr1R(SphI)::ura4 } 4^{+} \text {ade6-210 his 3-D leu1-32 ura4-DS } / E+ \\
{[\text { JP2525 pREP81-Sir2 S23D N247A-3x V5] }}\end{array}$ & This Study \\
\hline PY9967 & 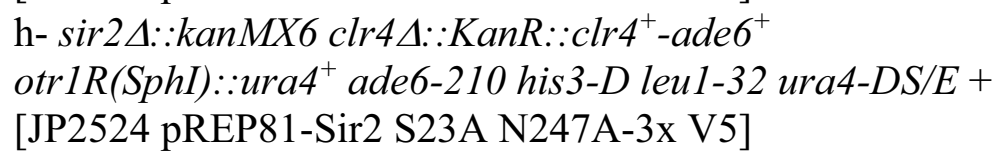 & This Study \\
\hline PY9970 & 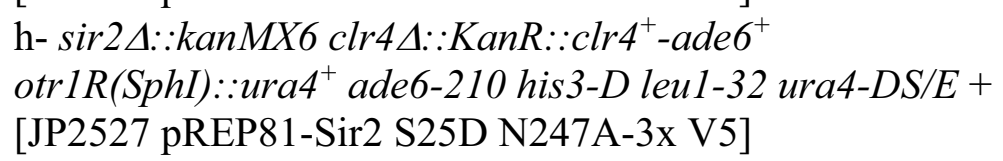 & This Study \\
\hline PY9973 & 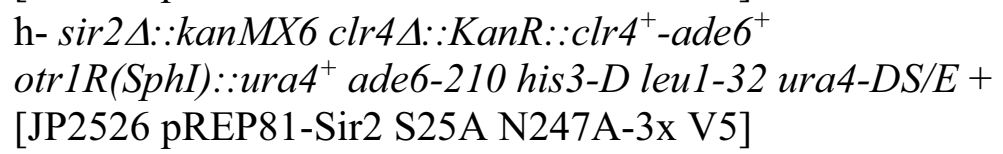 & This Study \\
\hline PY9976 & 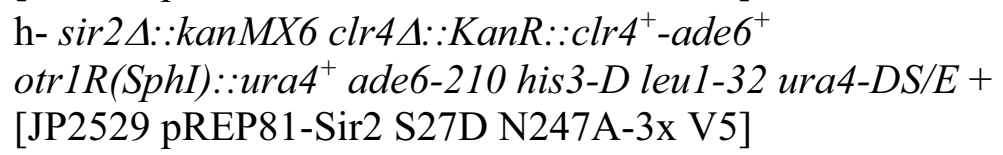 & This Study \\
\hline PY9979 & $\begin{array}{l}\text { h- } \operatorname{sir} 2 \Delta:: k a n M X 6 \text { clr4 } 4:: \text { KanR::clr } 4^{+} \text {-ade }^{+} \\
\text {otr1R(SphI)::ura4 }{ }^{+} \text {ade6-210 his3-D leul-32 ura4-DS/E+ } \\
{[\text { JP2528 pREP81-Sir2 S27A N247A-3x V5] }}\end{array}$ & This Study \\
\hline PY 10054 & $\begin{array}{l}\text { h- sir2 } 2:: \text { kanMX6 clr4 } 4:: \text { KanR }:: \text { clr }^{+} \text {-ade }^{+} \\
\text {otr1R(SphI)::ura4 } 4^{+} \text {ade6-210 his3-D leu1-32 ura4-DS/E+ } \\
{[\text { JP2540 pREP81-Sir2 S23, 25, 27A N247A-3x V5] }}\end{array}$ & This Study \\
\hline PY 10057 & 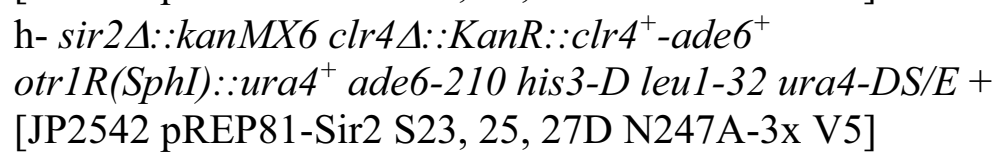 & This Study \\
\hline PY10116 & $\begin{array}{l}\text { h- } \operatorname{sir} 2 \Delta:: \text { kanMX6 clr4 } \Delta:: \text { KanR }:: \text { clr }^{+} \text {-ade } 6^{+} \\
\text {otr1R(SphI)::ura4 } 4^{+} \text {ade6-210 his3-D leu1-32 ura4-DS/E+ } \\
{[\text { JP2553 pREP81-Sir2 } \Delta 1-27 \text { N247A-3x V5] }}\end{array}$ & This Study \\
\hline
\end{tabular}


Table A-1. (Continued).

\begin{tabular}{|c|c|c|}
\hline Strain & Genotype & Source \\
\hline PY10119 & 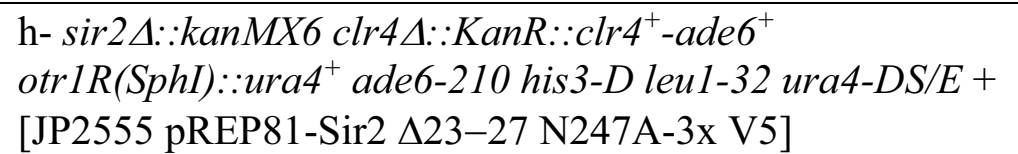 & This Study \\
\hline PY 10122 & 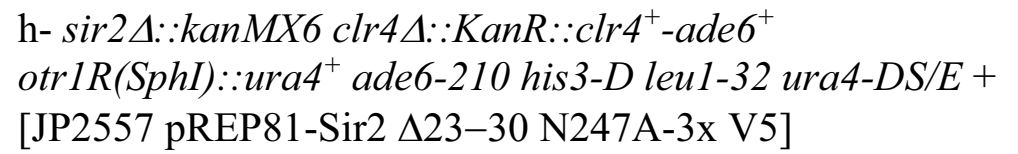 & This Study \\
\hline PY 10319 & $\begin{array}{l}\text { h- sir2 } \Delta:: k a n M X 6 \text { otr } 1 R\left(\text { Sph1)::ura4 } 4^{+} \text {ura4-DS/E ade6- }\right. \\
210 \text { leu1-32 his3-D + [JP2427 Prep81-Sir2 } \Delta 1-30-3 x \text { V5] }\end{array}$ & This Study \\
\hline PY 10322 & $\begin{array}{l}\text { h- sir2 } \Delta:: k a n M X 6 \text { otr } 1 R\left(\text { Sph1) }:: \text { ura }^{+} \text {ura } 4-D S / E \text { ade6- }\right. \\
210 \text { leu1-32 his3-D + [JP2432 Prep81-Sir2 } \Delta 1-60-3 x \text { V5] }\end{array}$ & This Study \\
\hline PY 10325 & $\begin{array}{l}\text { h- sir2 } \Delta:: k a n M X 6 \text { otr } 1 R\left(\text { Sph1)::ura4 }{ }^{+} \text {ura } 4-D S / E \text { ade6- }\right. \\
210 \text { leu1-32 his3-D + [JP2436 Prep81-Sir2 } \Delta 1-95-3 x \text { V5] }\end{array}$ & This Study \\
\hline PY10335 & $\begin{array}{l}\text { h- sir24::ura } 4^{+} \text {ade-210 arg3-D4 his3-D1 leu1-32 ura4- } \\
\text { D18 }\end{array}$ & This Study \\
\hline PY 10336 & $\begin{array}{l}\text { h- sir24::ura } 4^{+} \text {ade-210 arg3-D4 his3-D1 leu1-32 ura4- } \\
\text { D18 }\end{array}$ & This Study \\
\hline PY 10337 & $\begin{array}{l}\text { h- sir2 } 4:: \text { ura }^{+} \text {ade-210 arg3-D4 his3-D1 leu1-32 ura4- } \\
\text { D18 }\end{array}$ & This Study \\
\hline PY 10339 & h- sir2-3x V5 ade-210 arg3D4 his3-D1 leu1-32 ura4-D18 & This Study \\
\hline PY 10340 & h- sir2-3x V5 ade-210 arg3D4 his3-D1 leu1-32 ura4-D18 & This Study \\
\hline PY 10341 & $\begin{array}{l}\text { H90 sir2-3x V5 ade-210 arg3D4 his3-D1 leul-32 ura4- } \\
\text { D18 }\end{array}$ & This Study \\
\hline PY 10343 & $\begin{array}{l}\text { h- sir2 N247A-3x V5 ade-210 arg3-D4 his3-D1 leu1-32 } \\
\text { Ura4-D18 }\end{array}$ & This Study \\
\hline PY 10344 & $\begin{array}{l}\text { h- sir2 N247A-3x V5 ade-210 arg3-D4 his3-D1 leu1-32 } \\
\text { Ura4-D18 }\end{array}$ & This Study \\
\hline PY10442 & $\begin{array}{l}\text { h- sir2 } 41-129-3 x \text { V5 ade-210 arg3-D4 his3-D1 leu1-32 } \\
\text { ura4-D18 }\end{array}$ & This Study \\
\hline PY 10443 & $\begin{array}{l}\text { H90 sir2 } 41-129-3 x \text { V5 ade-210 arg3-D4 his3-D1 leu1-32 } \\
\text { ura4-D18 }\end{array}$ & This Study \\
\hline PY 10473 & h- sir2 $\Delta:: u r a 4^{+}$clr3 $\Delta:: k a n M X 6$ ade6-210 leu1-32 & This Study \\
\hline PY 10474 & h- sir2 $\Delta::$ ura $^{+}$clr3 $\Delta:: k a n M X 6$ ade6-210 leu1-32 & This Study \\
\hline PY 10582 & $\begin{array}{l}\text { h- sir2 } 2:: \text { ura }^{+} \text {clr1 } 1:: K a n M X 4 \text { arg3-D4 his3-D1 leu1-32 } \\
\text { ura4-D18 }\end{array}$ & This Study \\
\hline PY 10583 & $\begin{array}{l}\text { h- sir2 } 4:: \text { ura }^{+} \text {clr1 } 1:: K a n M X 4 \arg 3-D 4 \text { his3-D1 leu1-32 } \\
\text { ura4-D18 }\end{array}$ & This Study \\
\hline PY 10585 & $\begin{array}{l}\text { h- sir24::ura } 4^{+} \text {clr2::his } 7^{+} \text {arg3-D4 his3-D1 leu1-32 ura4- } \\
\text { D18 }\end{array}$ & This Study \\
\hline PY 10586 & $\begin{array}{l}\text { h- sir24::ura4 }{ }^{+} \operatorname{clr} 2:: \text { his } 7^{+} \text {arg3-D4 his3-D1 leul-32 ura4- } \\
\text { D18 }\end{array}$ & This Study \\
\hline PY 10588 & $\begin{array}{l}\text { h- } \operatorname{sir} 2 \Delta:: \text { ura }^{+} \operatorname{chp} 2 \Delta:: K a n R \text { ade6-210 arg3-D4 his3-D1 } \\
\text { leu1-32 ura4-D18 }\end{array}$ & This Study \\
\hline
\end{tabular}


Table A-1. (Continued).

\begin{tabular}{|c|c|c|}
\hline Strains & Genotype & Source \\
\hline PY 10589 & $\begin{array}{l}\text { h- sir2 } \Delta:: \text { ura }^{+} \operatorname{chp} 2 \Delta:: K a n R \text { ade6-210 arg3-D4 his3-D1 } \\
\text { leu1-32 ura4-D18 }\end{array}$ & This Study \\
\hline PY 10591 & $\begin{array}{l}\text { h- sir2 } \Delta:: \text { ura }^{+} \text {mit1 } \Delta:: N a t M X 6 \text { ade6-210 arg3-D4 his3- } \\
\text { D1 leu1-32 ura4-D18 }\end{array}$ & This Study \\
\hline PY 10592 & $\begin{array}{l}\text { h- sir2 } \Delta:: \text { ura }^{+} \text {mit1 } 1:: N a t M X 6 \text { ade6-210 arg3-D4 his3- } \\
\text { D1 leu1-32 ura4-D18 }\end{array}$ & This Study \\
\hline
\end{tabular}


Table A-2. Plasmids used in this study.

\begin{tabular}{|c|c|c|}
\hline Plasmid & Description & Source \\
\hline JP81 & pGEX-KG & $\mathrm{ATCC}^{\circledR}$ \\
\hline JP86 & pGEX-KG-GST-Chp1 CD & 85 \\
\hline JP1340 & pREP41 PkN & 194 \\
\hline JP1504 & pGEX-KG-Sir2 N247A & 83 \\
\hline JP1505 & pGEX-KG-Sir2 & 83 \\
\hline JP1611 & pREP81-3x V5 & 83 \\
\hline JP2102 & pREP41-Ded1-3x V5 & 157 \\
\hline JP2104 & pREP41-DDX3X-3x V5 & 157 \\
\hline JP2116 & pREP41-DDX3X G302V-3x V5 & 157 \\
\hline JP2132 & pREP41-DDX3X A222P-3x V5 & 157 \\
\hline JP2133 & pREP41-DDX3X G325E-3x V5 & 157 \\
\hline JP2134 & pREP41-DDX3X T275M-3x V5 & 157 \\
\hline JP2135 & pREP41-DDX3X R351W-3x V5 & 157 \\
\hline JP2136 & pREP41-DDX3X P568L-3x V5 & 157 \\
\hline JP2138 & pREP41-DDX3X K230A-3x V5 & 157 \\
\hline JP2139 & pREP41-DDX3X M370R-3x V5 & 157 \\
\hline JP2140 & pREP41-DDX3X D354V-3x V5 & 157 \\
\hline JP2183 & pREP41-DDX3X L353F-3x V5 & 157 \\
\hline JP2386 & pGEX-KG-Sir2 $\Delta 1-129$ & This Study \\
\hline JP2483 & pGEX-KG-GST-Sir2 1-128 & This Study \\
\hline JP2302 & pREP81-Sir2-3x V5 & This Study \\
\hline JP2304 & pREP81-Sir2 N247A-3x V5 & This Study \\
\hline JP2306 & pREP81-Sir2 $\Delta 1-129-3 x$ V5 & This Study \\
\hline JP2308 & pREP81-Sir2 $\Delta 1-129$ N247A-3x V5 & This Study \\
\hline JP2424 & pREP81-Sir2 $\Delta 1-20-3 x$ V5 & This Study \\
\hline JP2426 & pREP81-Sir2 $\Delta 1-20$ N247A-3x V5 & This Study \\
\hline JP2427 & pREP81-Sir2 $\Delta 1-30-3 x$ V5 & This Study \\
\hline JP2428 & pREP81-Sir2 $\Delta 1-30$ N247A-3x V5 & This Study \\
\hline JP2429 & pREP81-Sir2 $\Delta 1-36-3 x$ V5 & This Study \\
\hline JP2430 & pREP81-Sir2 $\Delta 1-36$ N247A-3x V5 & This Study \\
\hline JP2432 & pREP81-Sir2 $\Delta 1-60-3 x$ V5 & This Study \\
\hline JP2434 & pREP81-Sir2 $\Delta 1-60$ N247A-3x V5 & This Study \\
\hline JP2436 & pREP81-Sir2 $\Delta 1-95-3 x$ V5 & This Study \\
\hline JP2438 & pREP81-Sir2 $\Delta 1-95$ N247A-3x V5 & This Study \\
\hline JP2439 & pREP81-Sir2 $\Delta 23-36-3 x$ V5 & This Study \\
\hline JP2440 & pREP81-Sir2 $\Delta 23-36$ N247A-3x V5 & This Study \\
\hline JP2522 & pREP81-Sir2 T13A N247A-3x V5 & This Study \\
\hline JP2523 & pREP81-Sir2 T13D N247A-3x V5 & This Study \\
\hline JP2524 & pREP81-Sir2 T23A N247A-3x V5 & This Study \\
\hline JP2525 & pREP81-Sir2 T23D N247A-3x V5 & This Study \\
\hline
\end{tabular}


Table A-2. (Continued).

\begin{tabular}{lll}
\hline Plasmid & Description & Source \\
\hline JP2526 & pREP81-Sir2 T25A N247A-3x V5 & This Study \\
JP2527 & pREP81-Sir2 T25D N247A-3x V5 & This Study \\
JP2528 & pREP81-Sir2 T27A N247A-3x V5 & This Study \\
JP2529 & pREP81-Sir2 T27D N247A-3x V5 & This Study \\
JP2540 & pREP81-Sir2 T23,25,27A N247A-3x V5 & This Study \\
JP2542 & pREP81-Sir2 T23,25,27D N247A-3x V5 & This Study \\
JP2553 & pREP81-Sir2 $\Delta 1-27$ N247A-3x V5 & This Study \\
JP2555 & pREP81-Sir2 $\Delta 23-27$ N247A-3x V5 & This Study \\
\hline
\end{tabular}


Table A-3. Detection parameters of unique tryptic peptides from pombe $\mathbf{H 3}$.

\begin{tabular}{|c|c|c|c|c|}
\hline Peptide & $\begin{array}{l}\text { Precursor } \\
\text { Ion }(\mathbf{m} / \mathbf{z})\end{array}$ & $\begin{array}{l}\text { Product } \\
\text { Ions }(\mathrm{m} / \mathrm{z})\end{array}$ & $\begin{array}{l}\text { Collision } \\
\text { Energy }(e V)\end{array}$ & $\begin{array}{l}\text { Retention } \\
\text { Time (min) }\end{array}$ \\
\hline \multirow[t]{2}{*}{ KpQLASKpAAR } & 542.827 & 772.468 & 19 & 4.35 \\
\hline & & 659.384 & 19 & \\
\hline \multirow[t]{2}{*}{ KpQLASKaAAR } & 535.819 & 758.452 & 19 & 3.50 \\
\hline & & 645.368 & 19 & \\
\hline \multirow[t]{2}{*}{ KaQLASKpAAR } & 535.821 & 772.468 & 19 & 3.50 \\
\hline & & 659.384 & 19 & \\
\hline \multirow[t]{2}{*}{ KaQLASKaAAR } & 528.812 & 758.452 & 19 & 2.45 \\
\hline & & 645.368 & 19 & \\
\hline \multirow{3}{*}{ KpAAPATGGVKpKpPHR } & 529.311 & 1259.722 & 22 & 5.25 \\
\hline & & 933.563 & 22 & \\
\hline & & 593.352 & 22 & \\
\hline \multirow[t]{3}{*}{ KpAAPATGGVKpKaPHR } & 524.638 & 1245.706 & 22 & 4.85 \\
\hline & & 919.547 & 22 & \\
\hline & & 579.336 & 22 & \\
\hline \multirow[t]{3}{*}{ KpAAPATGGVKaKpPHR } & 524.640 & 1245.706 & 22 & 4.85 \\
\hline & & 919.547 & 22 & \\
\hline & & 593.352 & 22 & \\
\hline \multirow[t]{3}{*}{ KaAAPATGGVKpKpPHR } & 524.642 & 1259.722 & 22 & 4.85 \\
\hline & & 933.563 & 22 & \\
\hline & & 593.352 & 22 & \\
\hline \multirow[t]{3}{*}{ KpAAPATGGVKaKaPHR } & 519.967 & 1231.691 & 22 & 4.35 \\
\hline & & 905.532 & 22 & \\
\hline & & 579.336 & 22 & \\
\hline \multirow[t]{3}{*}{ KaAAPATGGVKpKaPHR } & 519.969 & 1245.706 & 22 & 4.35 \\
\hline & & 919.547 & 22 & \\
\hline & & 579.336 & 22 & \\
\hline \multirow[t]{3}{*}{ KaAAPATGGVKaKpPHR } & 519.971 & 1245.706 & 22 & 4.35 \\
\hline & & 919.547 & 22 & \\
\hline & & 593.352 & 22 & \\
\hline \multirow[t]{3}{*}{ KaAAPATGGVKaKaPHR } & 515.295 & 1231.691 & 22 & 3.80 \\
\hline & & 905.532 & 22 & \\
\hline & & 579.336 & 22 & \\
\hline \multirow[t]{4}{*}{ VTIQPKpDMQLAR } & 485.938 & 1014.540 & 21 & 8.85 \\
\hline & & 917.487 & 21 & \\
\hline & & 733.366 & 21 & \\
\hline & & 618.339 & 21 & \\
\hline \multirow[t]{4}{*}{ VTIQPKaDMQLAR } & 481.266 & 1000.524 & 21 & 8.75 \\
\hline & & 903.472 & 21 & \\
\hline & & 733.366 & 21 & \\
\hline & & 618.339 & 21 & \\
\hline
\end{tabular}


Table A-3. (Continued).

Notes: Propionylated lysines are represented by $\mathrm{p}$ while acetylated lysines are represented by the letter a.

Source: Unpublished communication from Dr. Andrew J. Andrews on 12/07/2016. 
Table A-4. Sir2-3x V5 and Sir2 $\Delta 1-129-3 x$ V5 MS/MS results.

\begin{tabular}{|c|c|c|c|}
\hline \multirow[b]{3}{*}{ Identified Proteins (301) } & \multicolumn{3}{|c|}{$\begin{array}{l}\text { Number of Peptide } \\
\text { Identified }\end{array}$} \\
\hline & & WT & Sir2 \\
\hline & $\operatorname{sir} 2 \Delta$ & Sir2 & $\Delta 1-129$ \\
\hline NAD-dependent histone deacetylase sir2 & 2 & 317 & 262 \\
\hline Phosphatidylinositol 3-kinase tor2 & 205 & 96 & 145 \\
\hline Fatty acid synthase subunit alpha & 51 & 69 & 88 \\
\hline Fatty acid synthase subunit beta & 43 & 60 & 89 \\
\hline Cluster of Probable heat shock protein ssa 2 & 47 & 60 & 96 \\
\hline Actin & 58 & 60 & 54 \\
\hline $\begin{array}{l}\text { Uncharacterized transcriptional regulatory protein } \\
\text { C3C7.04 }\end{array}$ & 56 & 51 & 55 \\
\hline Probable clathrin heavy chain & 40 & 40 & 51 \\
\hline Heat shock protein sks2 & 11 & 37 & 51 \\
\hline Ubiquitin-40S ribosomal protein $\mathrm{S} 27 \mathrm{a}$ & 25 & 37 & 35 \\
\hline Elongation factor 1-alpha-A & 49 & 36 & 44 \\
\hline Cluster of $60 \mathrm{~S}$ ribosomal protein L4-A & 39 & 34 & 37 \\
\hline $\begin{array}{l}\text { Cluster of Glyceraldehyde-3-phosphate dehydrogenase } \\
1\end{array}$ & 38 & 29 & 32 \\
\hline 60 S ribosomal protein $\mathrm{L} 7-\mathrm{C}$ & 23 & 28 & 19 \\
\hline $60 \mathrm{~S}$ ribosomal protein $\mathrm{L} 3-\mathrm{A}$ & 29 & 28 & 29 \\
\hline ATP-dependent 6-phosphofructokinase & 24 & 26 & 36 \\
\hline Heat shock protein homolog pss 1 & 4 & 25 & 30 \\
\hline 60S ribosomal protein L36-A & 13 & 25 & 23 \\
\hline 60 S ribosomal protein L5-A & 19 & 25 & 19 \\
\hline WD repeat-containing protein mip1 & 35 & 25 & 35 \\
\hline Cluster of $60 \mathrm{~S}$ ribosomal protein L28-B & 14 & 24 & 21 \\
\hline Cluster of 40 S ribosomal protein S1-B & 15 & 24 & 17 \\
\hline Probable 60 S ribosomal protein L28e & 12 & 23 & 19 \\
\hline $60 \mathrm{~S}$ ribosomal protein $\mathrm{L} 8$ & 21 & 23 & 24 \\
\hline Histone H4 & 17 & 22 & 25 \\
\hline Importin subunit alpha-2 & 0 & 19 & 20 \\
\hline 60 S ribosomal protein L23-A & 4 & 19 & 8 \\
\hline $40 \mathrm{~S}$ ribosomal protein $\mathrm{S} 2$ & 4 & 19 & 9 \\
\hline $\begin{array}{l}\text { Pyruvate dehydrogenase E1 component subunit alpha, } \\
\text { mitochondrial }\end{array}$ & 14 & 19 & 15 \\
\hline Cluster of $60 \mathrm{~S}$ ribosomal protein L18-B & 21 & 19 & 23 \\
\hline UPF0590 protein C594.01 & 22 & 19 & 24 \\
\hline Protein ura1 & 13 & 18 & 23 \\
\hline Cluster of $60 \mathrm{~S}$ ribosomal protein L1-B & 14 & 18 & 24 \\
\hline Cluster of 40S ribosomal protein S4-B & 14 & 18 & 15 \\
\hline
\end{tabular}


Table A-4. (Continued).

\begin{tabular}{|c|c|c|c|}
\hline \multirow[b]{2}{*}{ Identified Proteins (301) } & \multicolumn{3}{|c|}{$\begin{array}{c}\text { Number of Peptide } \\
\text { Identified }\end{array}$} \\
\hline & $\operatorname{sir} 2 \Delta$ & $\begin{array}{l}\text { WT } \\
\text { Sir2 }\end{array}$ & $\begin{array}{l}\text { Sir2 } \\
\Delta 1-129 \\
\end{array}$ \\
\hline Cluster of $60 \mathrm{~S}$ ribosomal protein L27-A & 20 & 18 & 23 \\
\hline 60 S ribosomal protein L38-1 & 10 & 17 & 12 \\
\hline $60 \mathrm{~S}$ ribosomal protein L33-B & 11 & 17 & 14 \\
\hline Histone H2A.Z & 14 & 16 & 12 \\
\hline Histone H2B-alpha & 10 & 15 & 11 \\
\hline Probable pyruvate decarboxylase C1F8.07c & 26 & 15 & 29 \\
\hline $60 \mathrm{~S}$ ribosomal protein $\mathrm{L} 43-\mathrm{B}$ & 8 & 14 & 18 \\
\hline $60 \mathrm{~S}$ ribosomal protein L33-A & 10 & 14 & 12 \\
\hline 60S ribosomal protein L14 & 13 & 14 & 17 \\
\hline Alcohol dehydrogenase & 14 & 14 & 16 \\
\hline $60 \mathrm{~S}$ ribosomal protein $\mathrm{L} 6$ & 14 & 14 & 21 \\
\hline $60 \mathrm{~S}$ acidic ribosomal protein $\mathrm{P} 0$ & 15 & 14 & 16 \\
\hline $\begin{array}{l}\text { Probable 5-methyltetrahydropteroyltriglutamate-- } \\
\text { homocysteine HMT }\end{array}$ & 29 & 14 & 18 \\
\hline $40 \mathrm{~S}$ ribosomal protein $\mathrm{S} 23-\mathrm{A}$ & 7 & 13 & 15 \\
\hline $60 \mathrm{~S}$ ribosomal protein $\mathrm{L} 30-1$ & 7 & 13 & 11 \\
\hline 40S ribosomal protein S6-B & 8 & 13 & 8 \\
\hline Cluster of $60 \mathrm{~S}$ ribosomal protein L10-A & 9 & 13 & 14 \\
\hline 60 S ribosomal protein L20-A & 14 & 13 & 19 \\
\hline Heat shock protein 90 homolog & 18 & 13 & 26 \\
\hline RuvB-like helicase 2 & 0 & 12 & 5 \\
\hline UPF0202 protein C20G8.09c & 0 & 12 & 0 \\
\hline $60 \mathrm{~S}$ ribosomal protein L38-2 & 5 & 12 & 7 \\
\hline $60 \mathrm{~S}$ ribosomal protein L11-A & 6 & 12 & 12 \\
\hline 60 S ribosomal protein L22 & 8 & 12 & 10 \\
\hline Cluster of 60 S ribosomal protein L32-B & 9 & 12 & 11 \\
\hline Histone H3.1/H3.2 & 9 & 12 & 8 \\
\hline 60 S ribosomal protein L12-A & 14 & 12 & 10 \\
\hline Uncharacterized protein P18G5.03 & 18 & 12 & 22 \\
\hline Pyruvate kinase & 41 & 12 & 16 \\
\hline Calnexin-independence factor 1 & 0 & 11 & 1 \\
\hline 40S ribosomal protein S15-B & 1 & 11 & 2 \\
\hline $60 \mathrm{~S}$ ribosomal protein L26 & 8 & 11 & 13 \\
\hline $40 \mathrm{~S}$ ribosomal protein $\mathrm{S} 28-\mathrm{A}$ & 10 & 11 & 18 \\
\hline $40 \mathrm{~S}$ ribosomal protein $\mathrm{S} 17-\mathrm{A}$ & 11 & 11 & 8 \\
\hline Cluster of 60 S ribosomal protein L17-B & 12 & 11 & 10 \\
\hline Cluster of $60 \mathrm{~S}$ ribosomal protein L15-A & 15 & 11 & 17 \\
\hline
\end{tabular}


Table A-4. (Continued).

\begin{tabular}{|c|c|c|c|}
\hline \multirow[b]{2}{*}{ Identified Proteins (301) } & \multicolumn{3}{|c|}{$\begin{array}{c}\text { Number of Peptide } \\
\text { Identified }\end{array}$} \\
\hline & $\operatorname{sir} 2 \Delta$ & $\begin{array}{l}\text { WT } \\
\text { Sir2 }\end{array}$ & $\begin{array}{l}\text { Sir2 } \\
\Delta 1-129\end{array}$ \\
\hline Cluster of 60 S ribosomal protein L19-B & 16 & 11 & 16 \\
\hline Elongation factor 2 & 16 & 11 & 15 \\
\hline 40S ribosomal protein $\mathrm{S} 27$ & 4 & 10 & 4 \\
\hline 40S ribosomal protein S24-B & 4 & 10 & 10 \\
\hline $60 \mathrm{~S}$ acidic ribosomal protein $\mathrm{P} 2-\mathrm{C}$ & 6 & 10 & 9 \\
\hline $78 \mathrm{kDa}$ glucose-regulated protein homolog & 9 & 10 & 18 \\
\hline Probable serine hydroxymethyltransferase, cytosolic & 10 & 10 & 7 \\
\hline $60 \mathrm{~S}$ ribosomal protein $\mathrm{L} 34-\mathrm{A}$ & 11 & 10 & 5 \\
\hline RuvB-like helicase 1 & 0 & 9 & 4 \\
\hline 40S ribosomal protein S15-A & 1 & 9 & 2 \\
\hline ATP-dependent RNA helicase ded1 & 6 & 9 & 17 \\
\hline ATP synthase subunit beta, mitochondrial & 9 & 9 & 7 \\
\hline 60 S ribosomal protein L13 & 10 & 9 & 8 \\
\hline WD repeat-containing protein wat 1 & 12 & 9 & 14 \\
\hline Importin subunit beta-1 & 0 & 8 & 8 \\
\hline 26S protease regulatory subunit 7 homolog & 0 & 8 & 1 \\
\hline Cluster of Probable $26 \mathrm{~S}$ protease subunit rpt4 & 1 & 8 & 6 \\
\hline Dihydrolipoyl dehydrogenase, mitochondrial & 3 & 8 & 9 \\
\hline Cell division cycle protein 48 & 4 & 8 & 17 \\
\hline $40 \mathrm{~S}$ ribosomal protein $\mathrm{S} 11-\mathrm{A}$ & 4 & 8 & 5 \\
\hline Cluster of 60 S ribosomal protein L25-A & 6 & 8 & 5 \\
\hline Cluster of 60 S ribosomal protein L37-B & 7 & 8 & 8 \\
\hline DNA damage checkpoint protein $\operatorname{rad} 24$ & 7 & 8 & 14 \\
\hline $40 \mathrm{~S}$ ribosomal protein $\mathrm{S} 9-\mathrm{A}$ & 8 & 8 & 12 \\
\hline S-adenosylmethionine synthase & 8 & 8 & 7 \\
\hline $40 \mathrm{~S}$ ribosomal protein $\mathrm{S} 3$ & 9 & 8 & 10 \\
\hline $\begin{array}{l}\text { Pyruvate dehydrogenase E1 component subunit beta, } \\
\text { mitochondrial }\end{array}$ & 10 & 8 & 7 \\
\hline $60 \mathrm{~S}$ ribosomal protein $\mathrm{L} 35$ & 11 & 8 & 18 \\
\hline $60 \mathrm{~S}$ ribosomal protein $\mathrm{L} 2-\mathrm{A}$ & 11 & 8 & 14 \\
\hline 60 S ribosomal protein $\mathrm{L} 7-\mathrm{B}$ & 11 & 8 & 11 \\
\hline Protein efr3 & 0 & 7 & 0 \\
\hline Tripeptidyl-peptidase 2 homolog & 3 & 7 & 4 \\
\hline Cluster of $60 \mathrm{~S}$ acidic ribosomal protein $\mathrm{P} 1$-alpha 1 & 3 & 7 & 4 \\
\hline 60 S ribosomal protein $\mathrm{L} 30-2$ & 4 & 7 & 5 \\
\hline $40 \mathrm{~S}$ ribosomal protein $\mathrm{S} 20$ & 5 & 7 & 9 \\
\hline Cluster of 40 S ribosomal protein S19-B & 5 & 7 & 9 \\
\hline
\end{tabular}


Table A-4. (Continued).

\begin{tabular}{|c|c|c|c|}
\hline \multirow[b]{3}{*}{ Identified Proteins (301) } & \multicolumn{3}{|c|}{$\begin{array}{c}\text { Number of Peptide } \\
\text { Identified }\end{array}$} \\
\hline & & $\mathbf{W T}$ & Sir2 \\
\hline & $\operatorname{sir} 2 \Delta$ & Sir2 & $\Delta 1-129$ \\
\hline Putative nucleosome assembly protein C364.06 & 6 & 7 & 7 \\
\hline Polyadenylate-binding protein, cytoplasmic and nuclear & 6 & 7 & 7 \\
\hline $26 \mathrm{~S}$ protease regulatory subunit 8 homolog & 6 & 7 & 7 \\
\hline $40 \mathrm{~S}$ ribosomal protein $\mathrm{S} 18-\mathrm{A}$ & 8 & 7 & 6 \\
\hline Enolase $1-1$ & 22 & 7 & 10 \\
\hline Probable methionine--tRNA ligase, cytoplasmic & 0 & 6 & 3 \\
\hline Switch-activating protein 1 & 0 & 6 & 2 \\
\hline Tyrosine-protein phosphatase 3 & 0 & 6 & 0 \\
\hline $26 \mathrm{~S}$ proteasome regulatory subunit rpn1 & 2 & 6 & 7 \\
\hline 40S ribosomal protein $\mathrm{S} 10-\mathrm{B}$ & 2 & 6 & 5 \\
\hline $40 \mathrm{~S}$ ribosomal protein $\mathrm{S} 22-\mathrm{A}$ & 3 & 6 & 6 \\
\hline $40 \mathrm{~S}$ ribosomal protein $\mathrm{S} 24-\mathrm{A}$ & 3 & 6 & 6 \\
\hline 40S ribosomal protein $\mathrm{S} 5-\mathrm{A}$ & 5 & 6 & 7 \\
\hline 40S ribosomal protein $\mathrm{S} 14-\mathrm{A}$ & 5 & 6 & 2 \\
\hline DNA damage checkpoint protein $\operatorname{rad} 25$ & 5 & 6 & 10 \\
\hline 40S ribosomal protein $\mathrm{S} 26-\mathrm{A}$ & 5 & 6 & 5 \\
\hline 40S ribosomal protein $\mathrm{S} 8-\mathrm{B}$ & 6 & 6 & 7 \\
\hline Eukaryotic translation initiation factor 3 subunit B & 8 & 6 & 9 \\
\hline Thiamine thiazole synthase & 11 & 6 & 16 \\
\hline Phosphoglycerate kinase & 28 & 6 & 11 \\
\hline $26 \mathrm{~S}$ protease regulatory subunit $6 \mathrm{~A}$ & 0 & 5 & 1 \\
\hline $\begin{array}{l}\text { Probable sphingolipid long chain base-responsive } \\
\text { protein pill }\end{array}$ & 0 & 5 & 1 \\
\hline $60 \mathrm{~S}$ ribosomal protein L24-A & 1 & 5 & 6 \\
\hline Protein slt1 & 1 & 5 & 3 \\
\hline Nucleoporin nup211 & 1 & 5 & 2 \\
\hline Acid phosphatase & 2 & 5 & 8 \\
\hline 60 S ribosomal protein L39 & 2 & 5 & 8 \\
\hline Heat shock protein 60 , mitochondrial & 4 & 5 & 25 \\
\hline Cluster of $40 \mathrm{~S}$ ribosomal protein $\mathrm{S} 0-\mathrm{A}$ & 5 & 5 & 11 \\
\hline Phosphatidylinositol 3-kinase tor1 & 7 & 5 & 6 \\
\hline ATP synthase subunit alpha, mitochondrial & 0 & 4 & 6 \\
\hline 40S ribosomal protein $\mathrm{S} 10-\mathrm{A}$ & 0 & 4 & 7 \\
\hline Putative DNA helicase ino80 & 0 & 4 & 1 \\
\hline Nucleolar protein 56 & 0 & 4 & 0 \\
\hline $\begin{array}{l}\text { Uncharacterized AAA domain-containing protein } \\
\text { C16E9.10c }\end{array}$ & 0 & 4 & 0 \\
\hline
\end{tabular}


Table A-4. (Continued).

\begin{tabular}{|c|c|c|c|}
\hline \multirow[b]{2}{*}{ Identified Proteins (301) } & \multicolumn{3}{|c|}{$\begin{array}{l}\text { Number of Peptide } \\
\text { Identified }\end{array}$} \\
\hline & $\operatorname{sir} 2 \Delta$ & $\begin{array}{l}\text { WT } \\
\text { Sir2 }\end{array}$ & $\begin{array}{l}\text { Sir2 } \\
\Delta 1-129\end{array}$ \\
\hline 40S ribosomal protein $\mathrm{S} 7$ & 1 & 4 & 4 \\
\hline Clathrin light chain & 1 & 4 & 4 \\
\hline $60 \mathrm{~S}$ ribosomal protein $\mathrm{L} 31$ & 1 & 4 & 3 \\
\hline Mannose-1-phosphate guanyltransferase & 1 & 4 & 2 \\
\hline $60 S$ acidic ribosomal protein $\mathrm{P} 1$-alpha 5 & 1 & 4 & 3 \\
\hline Eukaryotic translation initiation factor 3 subunit A & 3 & 4 & 7 \\
\hline $40 \mathrm{~S}$ ribosomal protein $\mathrm{S} 21$ & 3 & 4 & 4 \\
\hline $60 \mathrm{~S}$ acidic ribosomal protein $\mathrm{P} 2$-alpha & 3 & 4 & 4 \\
\hline 60 S ribosomal protein L16-B & 6 & 4 & 6 \\
\hline ATP-dependent RNA helicase dbp2 & 7 & 4 & 6 \\
\hline Putative aldehyde dehydrogenase-like protein C9E9.09c & 9 & 4 & 10 \\
\hline Uncharacterized protein $\mathrm{C} 162.12$ & 14 & 4 & 8 \\
\hline Importin subunit alpha-1 & 0 & 3 & 5 \\
\hline Actin-like protein arp5 & 0 & 3 & 0 \\
\hline Ribonucleoside-diphosphate reductase large chain & 0 & 3 & 2 \\
\hline Probable 26S proteasome regulatory subunit rpn6 & 0 & 3 & 2 \\
\hline DNA repair protein rad50 & 0 & 3 & 0 \\
\hline Histone deacetylase clr6 & 0 & 3 & 1 \\
\hline rRNA 2'-O-methyltransferase fibrillarin & 0 & 3 & 0 \\
\hline Suppressor of kinetochore protein 1 & 0 & 3 & 0 \\
\hline Putative peroxiredoxin pmp20 & 1 & 3 & 3 \\
\hline $\begin{array}{l}\text { Saccharopine dehydrogenase [NADP }(+) \text {, L-glutamate- } \\
\text { forming] }\end{array}$ & 1 & 3 & 1 \\
\hline $\begin{array}{l}\text { Probable pyruvate dehydrogenase protein X component, } \\
\text { mitochondrial }\end{array}$ & 2 & 3 & 5 \\
\hline Probable nucleolar GTP-binding protein 1 & 2 & 3 & 3 \\
\hline $26 \mathrm{~S}$ proteasome regulatory subunit rpn5 & 2 & 3 & 3 \\
\hline 3-isopropylmalate dehydrogenase & 2 & 3 & 2 \\
\hline $26 \mathrm{~S}$ protease regulatory subunit 4 homolog & 3 & 3 & 7 \\
\hline Peroxiredoxin tpx 1 & 5 & 3 & 3 \\
\hline $40 \mathrm{~S}$ ribosomal protein $\mathrm{S} 16-\mathrm{A}$ & 6 & 3 & 8 \\
\hline $\begin{array}{l}\text { Guanine nucleotide-binding protein subunit beta-like } \\
\text { protein }\end{array}$ & 6 & 3 & 6 \\
\hline Cluster of 40 S ribosomal protein $\mathrm{S} 12-\mathrm{A}$ & 7 & 3 & 7 \\
\hline Adenosylhomocysteinase & 12 & 3 & 5 \\
\hline
\end{tabular}

Source: Unpublished communication from Alan Tackett (UAMS) on 11/23/2015 
Table A-5. Phosphorylated Sir2 peptides obtained from LC-MS/MS.

\begin{tabular}{lll}
\hline Peptide & $\begin{array}{l}\text { Phosphorylation } \\
\text { Site(s) }\end{array}$ & $\begin{array}{l}\text { MS/MS } \\
\text { Technique }\end{array}$ \\
\hline (L)DNNMPTTPVEEKIPVASY(S) & T13phos & CID \\
(L)DNNMPTTPVEEKIPVASY(S) & T13phos & CID \\
(L)DNNMPTTPVEEKIPVASY(S) & T13phos & CID \\
(L)DNNMPTTPVEEKIPVASY(S) & T13phos & CID \\
(L)DNNMPTTPVEEKIPVASY(S) & T13phos & HCD \\
(L)DNNMPTTPVEEKIPVASY(S) & T13phos & HCD \\
(L)DNNMPTTPVEEKIPVASY(S) & T13phos & HCD \\
(L)DNNMPTTPVEEKIPVASY(S) & T13phos & HCD \\
(L)DNNMPTTPVEEKIPVASYSPSSSGSSSGASLL(V) & T13, S23phos & CID \\
(L)DNNMPTTPVEEKIPVASYSPSSSGSSSGASLL(V) & T13, S23phos & HCD \\
(L)DNNMPTTPVEEKIPVASYSPSSSGSSSGASLL(V) & T13, S23phos & HCD \\
(Y)SPSSSGSSSGASLL(V) & S25phos & CID \\
(Y)SPSSSGSSSGASLL(V) & S25phos & CID \\
(Y)SPSSSGSSSGASLL(V) & S27phos & HCD \\
(Y)FSDGDSESSEDDLAQPGIMKPDITF(F) & S329phos & CID \\
(Y)FSDGDSESSEDDLAQPGIMKPDITF(F) & S329phos & CID \\
(Y)FSDGDSESSEDDLAQPGIMKPDITF(F) & S329phos & HCD \\
\hline
\end{tabular}

Notes: CID stands for Collision-Induced Dissociation and HCD stands for High-Energy Collisional Dissociation.

Source: Unpublished communication from Alan Tackett (UAMS) on 10/01/2015. 


\section{VITA}

The son of parents Tim and Donna Lowe, Brandon Ray Lowe was born in Jackson, Tennessee in 1988. He graduated from West Carroll High School in 2006 before completing his Bachelor of Science degree in biology at Union University (Jackson, Tennessee) in the spring of 2010. In the fall of 2010, Brandon enrolled in the Integrated Program in the Biomedical Sciences at the University of Tennessee Health Science Center in Memphis, Tennessee. His dissertation work was performed under the supervision of Dr. Janet Partridge at St. Jude Children's Research Hospital in Memphis, Tennessee studying the processes guiding heterochromatin formation in fission yeast.

\section{Publications}

1. Alper, B.J.*, B.R. Lowe*, and J.F. Partridge, Centromeric heterochromatin assembly in fission yeast--balancing transcription, RNA interference and chromatin modification. Chromosome Res, 2012. 20(5): p. 521-34.

2. Alper, B.J., G. Job, R.K. Yadav, S. Shanker, B.R. Lowe, and J.F. Partridge, Sir2 is required for $\mathrm{Clr} 4$ to initiate centromeric heterochromatin assembly in fission yeast. Embo j, 2013. 32(17): p. 2321-35.

3. Kallappagoudar, S.*, R.K. Yadav*, B.R. Lowe*, and J.F. Partridge, Histone H3 mutations--a special role for H3.3 in tumorigenesis? Chromosoma, 2015. 124(2): p. 177-89.

4. Epling, L.B.*, C.R. Grace*, B.R. Lowe*, J.F. Partridge, and E.J. Enemark, Cancer-associated mutants of RNA helicase DDX3X are defective in RNAstimulated ATP hydrolysis. J Mol Biol, 2015. 427(9): p. 1779-96.

5. Job, G*., C. Brugger*, T. Xu*, B.R. Lowe*, Y. Pfister, C. Qu, S. Shanker, J.I. Banos Sanz, J.F. Partridge, and T. Schalch, SHREC Silences Heterochromatin via Distinct Remodeling and Deacetylation Modules. Mol Cell, 2016. 62(2): p. 20721.

Note: $(*)$ represents equal contribution. 